Fondazione

Teatro La Fenice di Venezia
Stagione 2012-2013

Lirica e Balletto

\title{
Richard Wagner
}

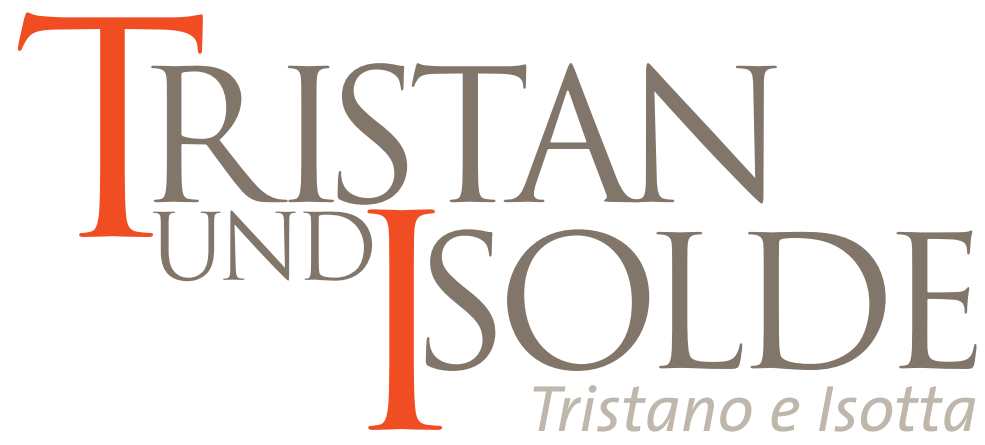




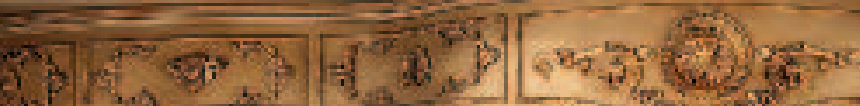

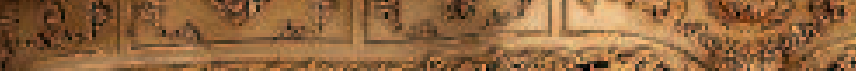

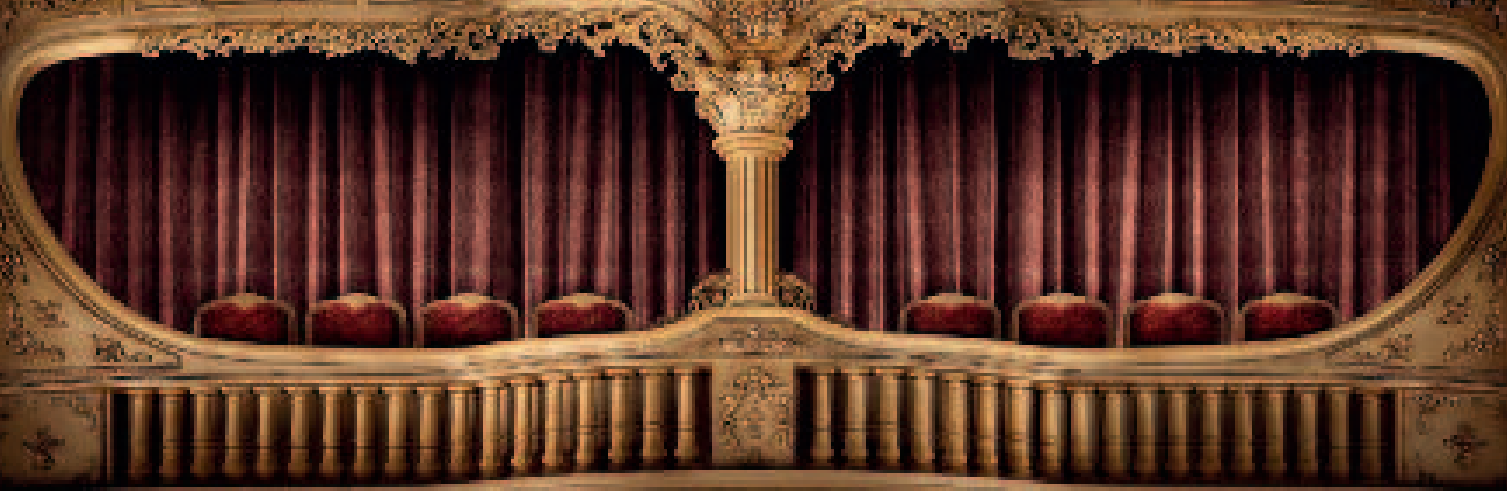

\section{QUESTA SERA,} BMW VI ACCOMPAGNA A TEATRO. BMW AL FIANCO DELLA GRANDE MUSICA. 


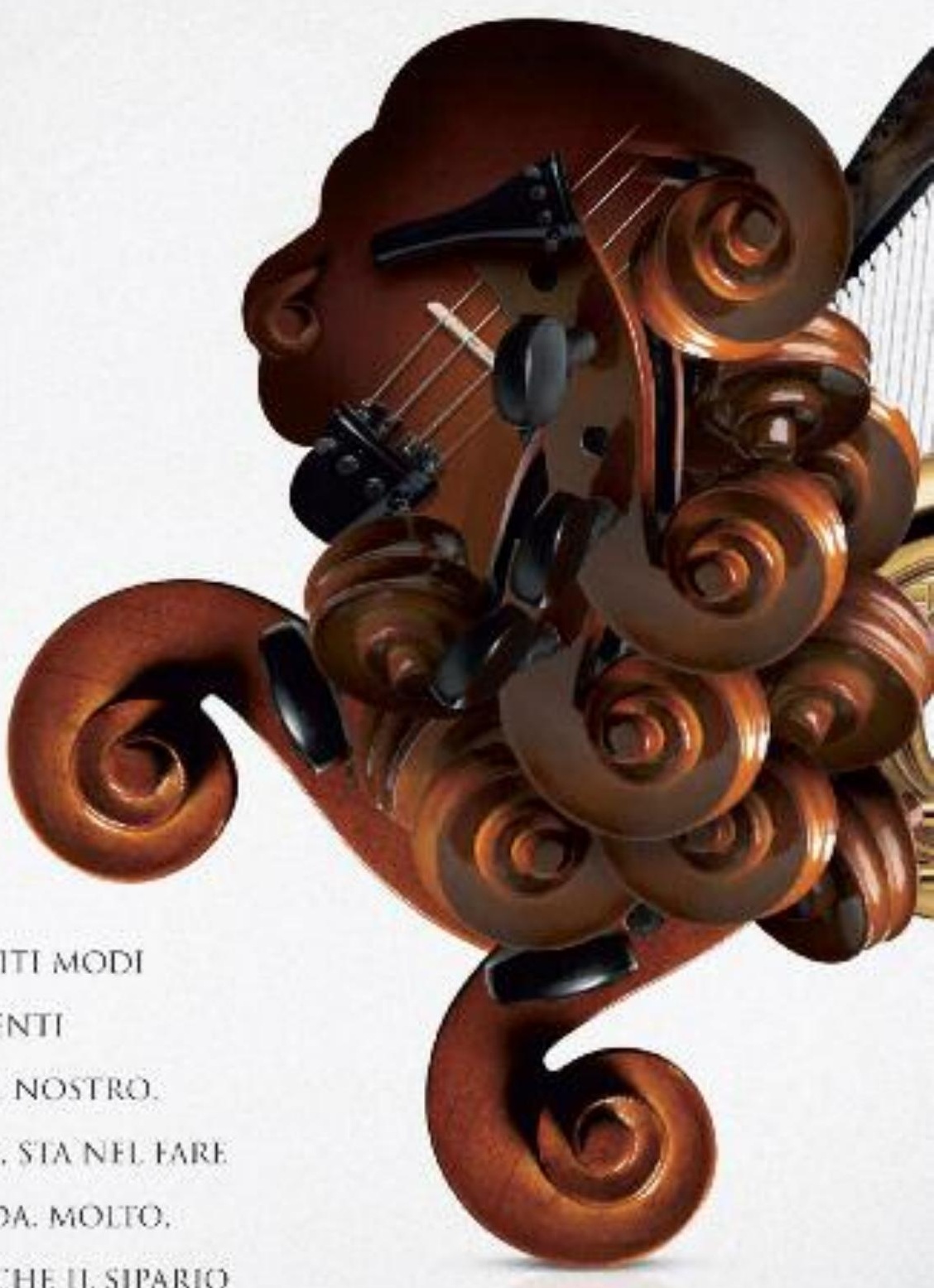

MOLTO PRIMA CHE IL SIPARIO

SI ALZI GENERALI E LI.

GENERALI. DOVE C'Ẽ ARTE.

DI FSSERF PRESFNTI

SULIA SCENA II NOSTRO.

SIORICAMENIE, SIA NFI HARE

CHE CIO ACCADA. MOLTO.

ARTE. 


\section{Se dovessicercare}

\section{una parola}

che sostituisce

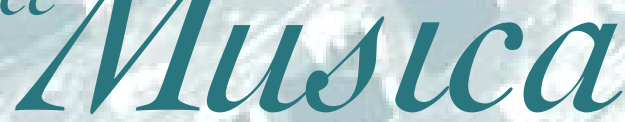

potrei pensare soltanto a

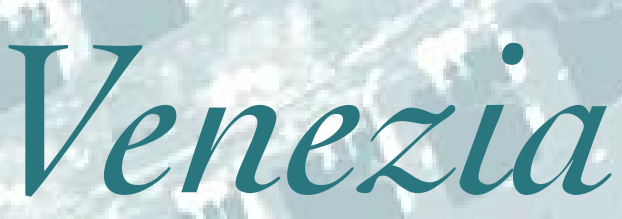

(Friedrich Nietzsche)

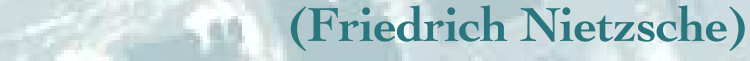

\section{Visita il Teatro La Fenice}

visite guidate

visite con audioguida

aperto tutti i giorni

dalle 9:30 alle 18:00

www.festfenice.com

Fest srl San Marco 4387, 30124 Venezia info@festfenice.com 


\section{The original factory. The original fabrics. The original Fortuny.}

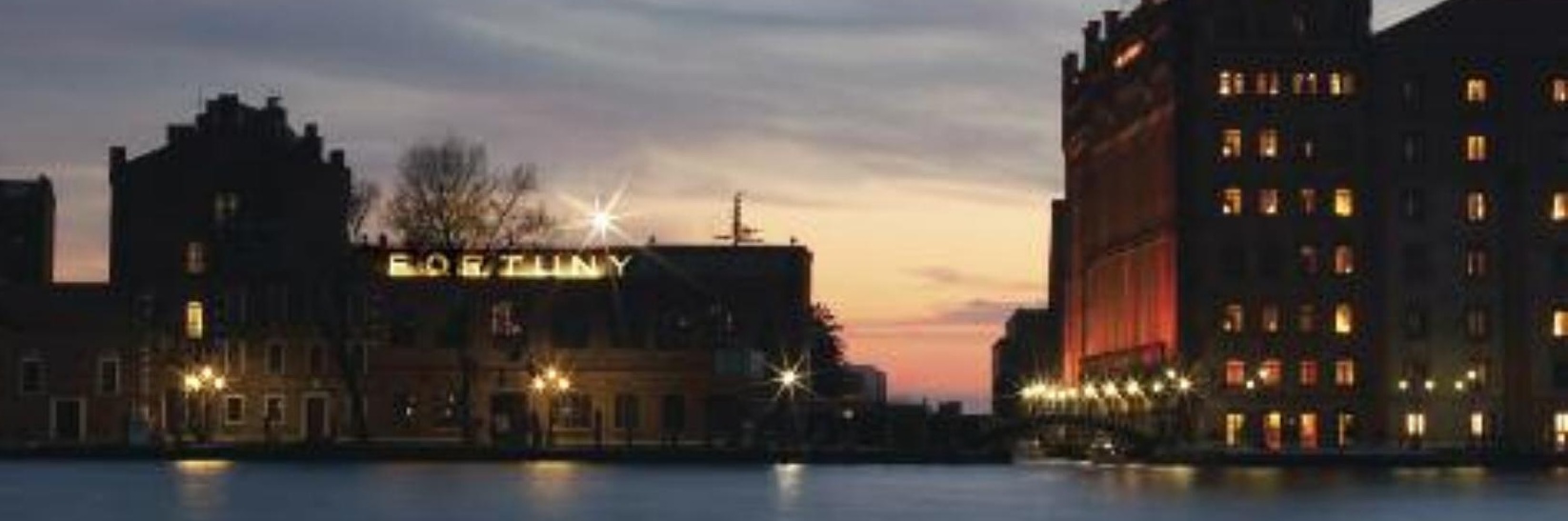

The only official Fortuny Showroom in Venice.

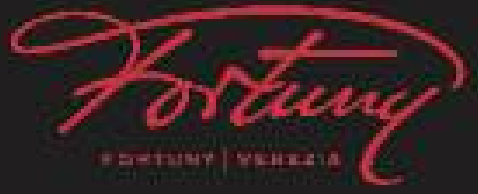




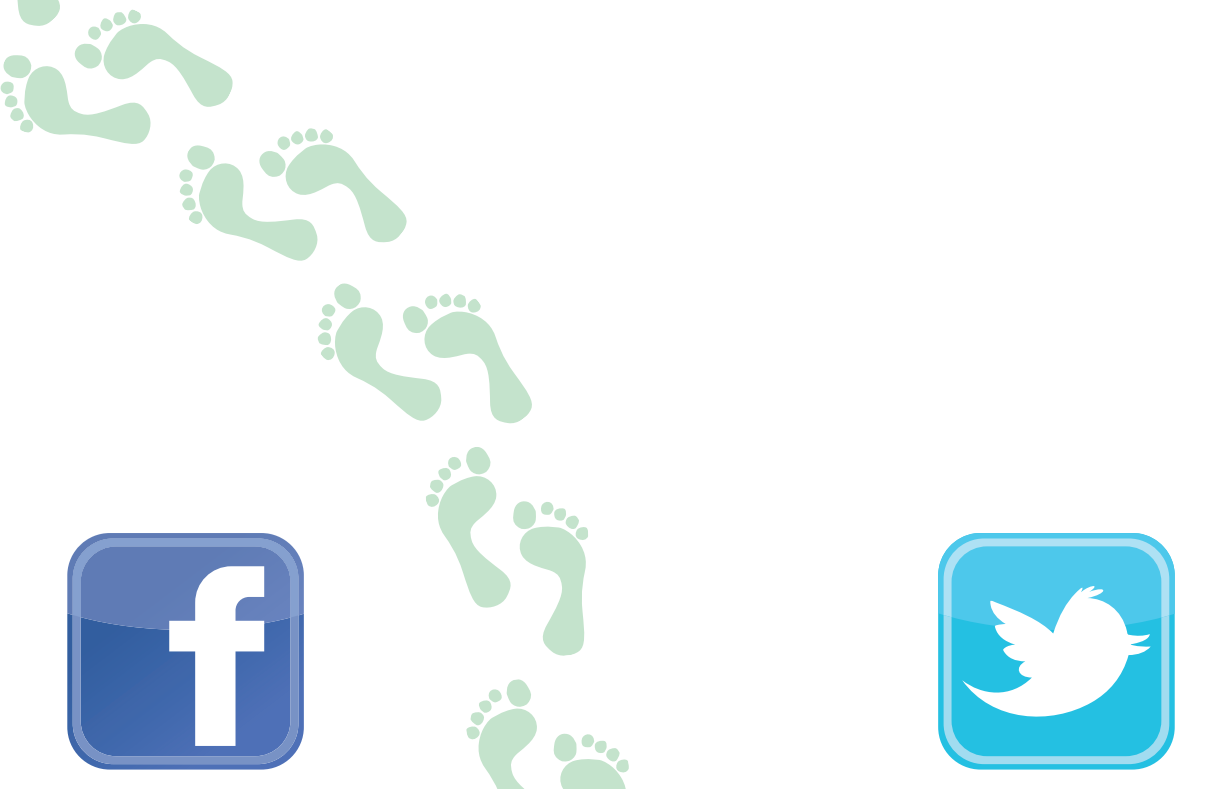

TEATRO LA FENICE - pagina ufficiale seguici su facebook, twitter e youtube follow us on facebook, twitter and youtube

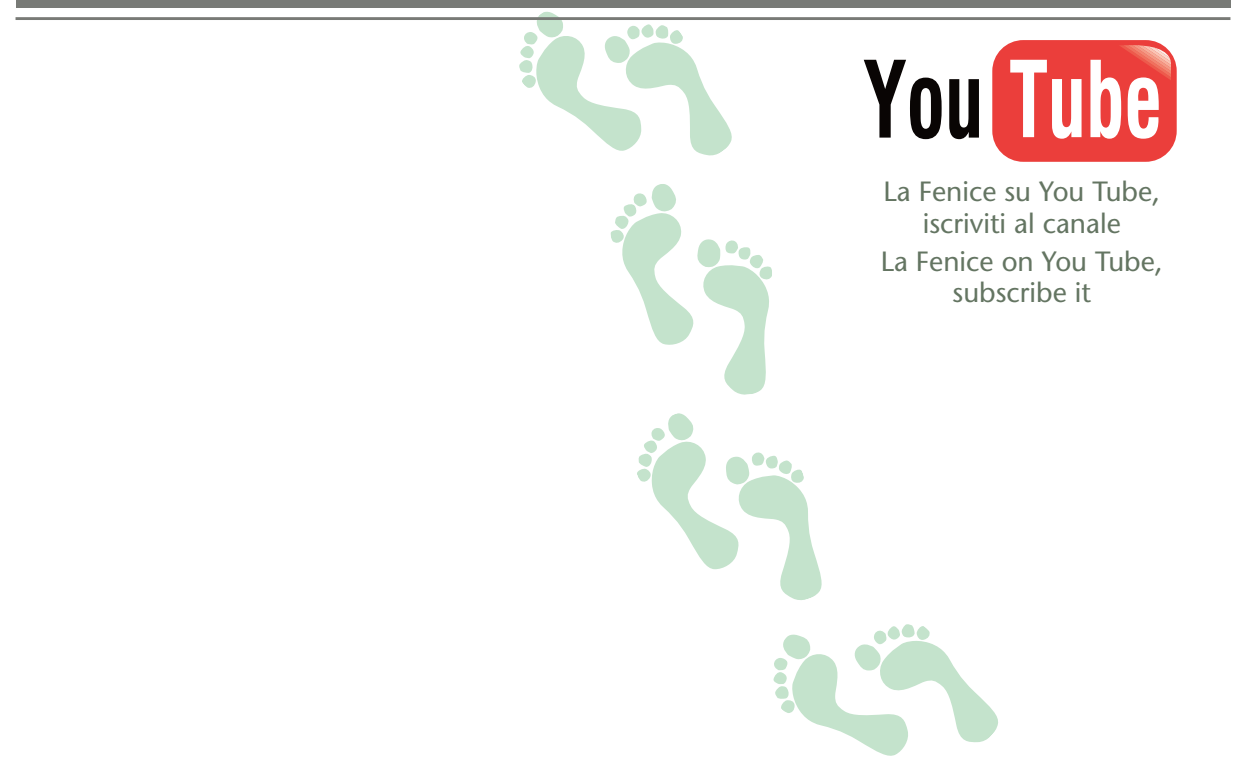




\section{CLASSICA \\ DIRETTORE SARAI TU.}

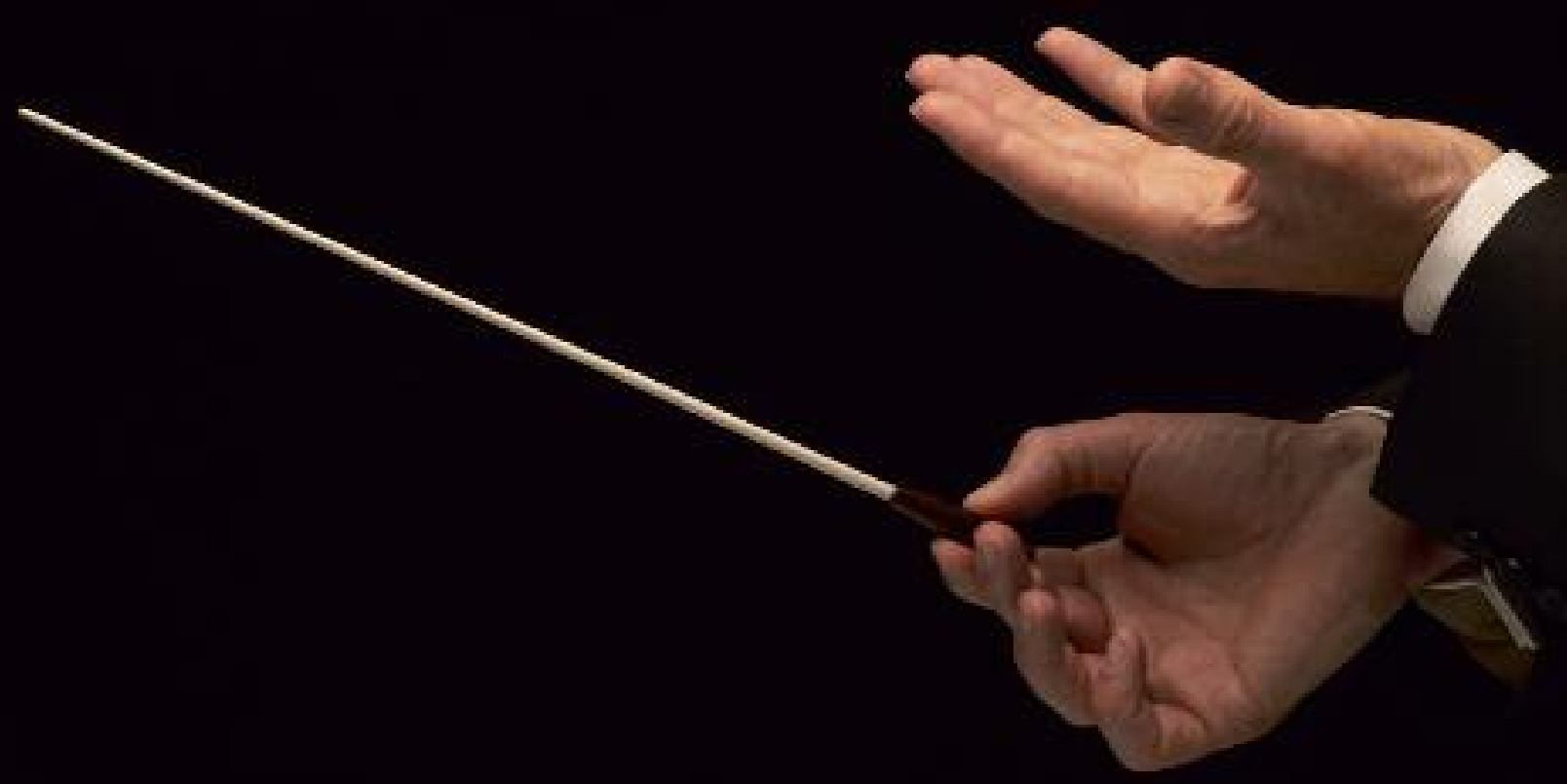

\section{ABBONATI A CLASSICA \\ IL CANALE TELEVISIVO DEDICATO ALLA GRANDE MUSICA}

AVRAI 24 ORE AL GIORNO DI:

- concerti sinfonici

- opere liriche

- danza classica e moderna

- musica da camera
- documentari

- musica contemporanea

- film, musical

- jazz

LA GRANDE MUSICA CONQUISTA IL PICCOLO SCHERMO
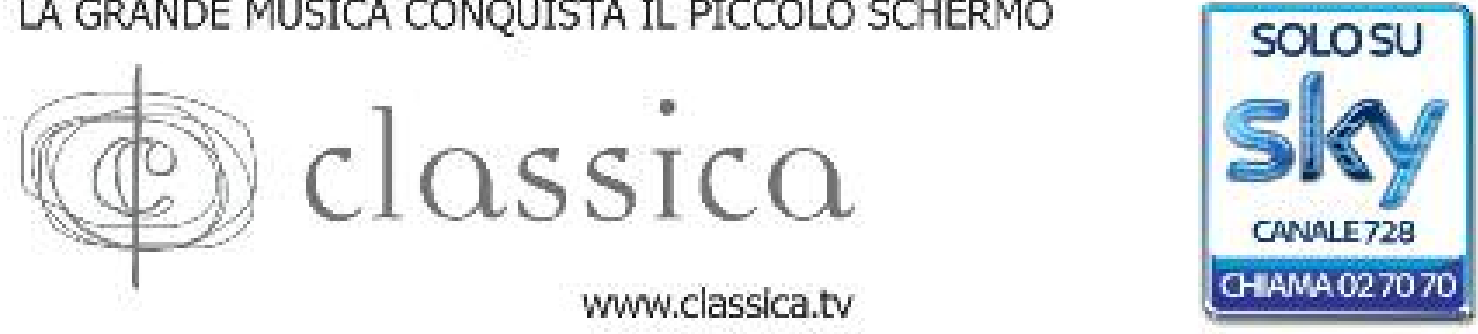


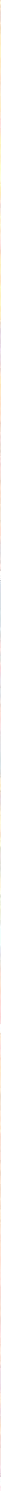




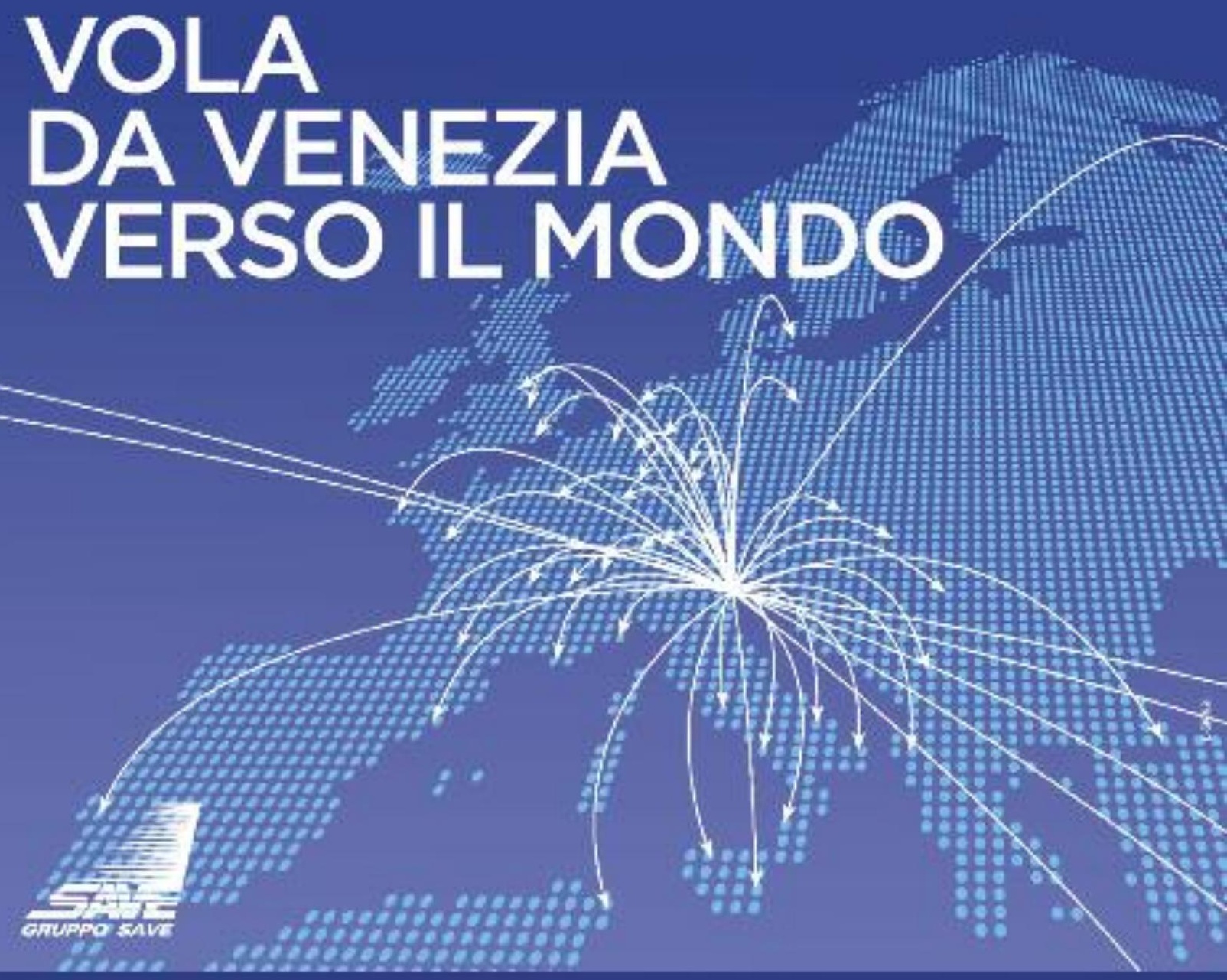

Scegll la destinazlone, vlaggla dove vuol

A

ALGHERO

AICANTE

AMSTERDAM

ATHENS

ATLANTA

B

BACAN

BARCELONA

BARI

BASEL

BERLIN

BLEAO

GRMINGHAM

GOROEAUX

BFATISEAVA

BR NDIS!

BRUSSELS

AUEHAZEST

SUDAPEST

C

CAGLIARI

CASABLANCA

CATANIS

CHISINAU
COLOGNE-BONN COPENHAGEN CORPL

D

DOHA

DUBAI

DUDLIS

DLERCWNKK

DUSSELDORF

E

FDIN⿴囗十⿵:5-

EINDHOVEN

$F$

FRANKFURT

$G$

GENEVE

H

HAMGURG

HANOWER

AELSINIKI

HERAKLION

I

$1812 \mathrm{~A}$

ISTANEUI

K
Kos

KRAKOW

L

LAMEZIA TERME

LAMPEOLSA

LEEOS-BRADFOIDO

LILLE

LISBON

LONDON LGW

LONDON LHZ

LUXEMBCURG

LYON

$M$

MADRID

MALAGA

MANCHESTER

MARSEILLE

MENORCA

MONTREAL

MOScow

MUNICH

MYKONOS

N

NANTFS

NAPLES
NEW YORK JFK

NEWCASTLE

Nicet

NOTTINGHAM

o

OLBIA

OELO

P

PALERMO

PALMA MALLORCA

PAFIS EIX:

PARIS ORY

PHILADELPHIA

PCRTO

PRAGUE

PFISTINA

R

REGGIO CALAERIA

Risa

$\mathrm{RODI}$

ROMA

5

SANTIAGO DEC.

STANTOFINI

5OFIA
SPLIT

ST. PETERSRURG

STOCKHOLM

STRASBOURC

STUTEGAT

$T$

TALLNV

THESSALONIKI

TIMISOARA

TIRANA

TORONTO

TOJLOUSE

TUNIS

$v$

VA-ENCIA

VA-LADOLID

VENNA

w

WAREAW

$Y$

YEFENAN

$z$

ZARAGOZA

ZNVICH 


\section{FONDAZIONE AMICI DELLA FENICE}

STAGIONE 2012-2013

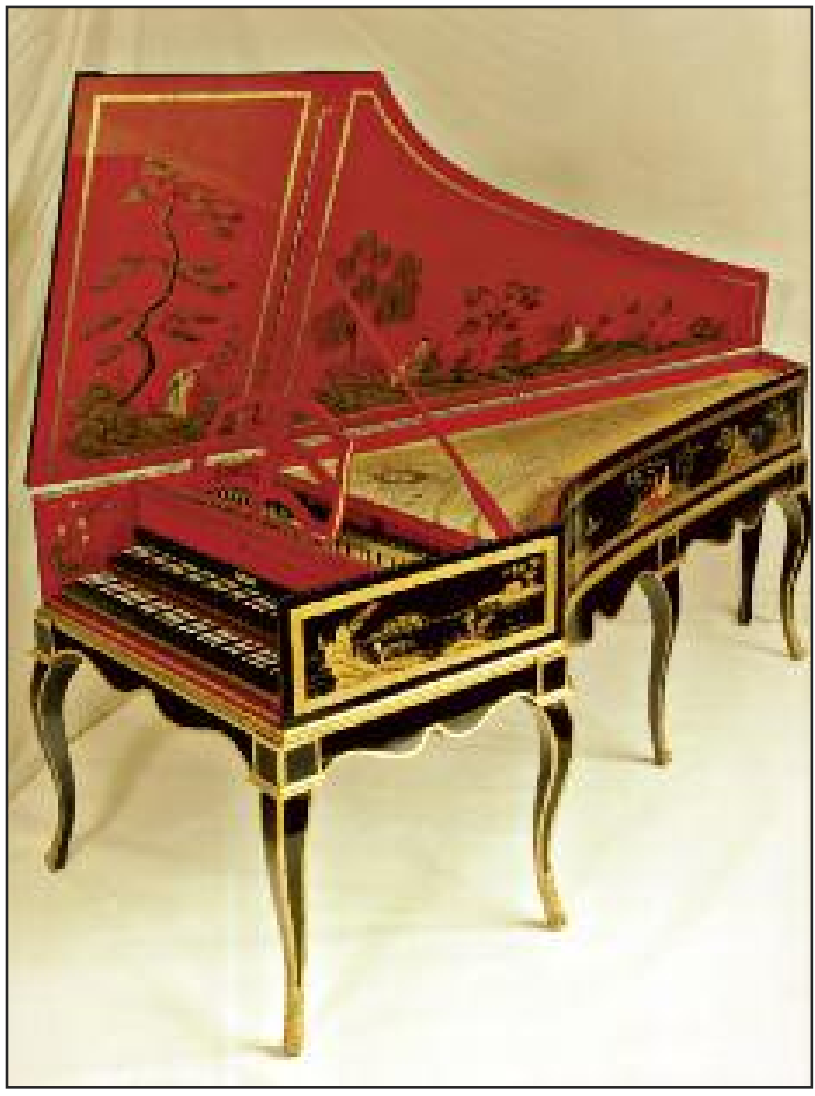

Clavicembalo francese a due manuali copia dello strumento di Goermans-Taskin, costruito attorno alla metà del XVIII secolo (originale presso la Russell Collection di Edimburgo).

Opera del $M^{\circ}$ cembalaro Luca Vismara di Seregno (MI); ultimato nel gennaio 1998.

Le decorazioni, la laccatura a tampone e le chinoiseries - che sono espressione di gusto tipicamente settecentesco per l'esotismo orientaleggiante, in auge soprattutto in ambito francese - sono state eseguite dal laboratorio dei fratelli Guido e Dario Tonoli di Meda (MI).

Caratteristiche tecniche: estensione $f a^{1}-f a^{5}$, trasposizione tonale da $415 \mathrm{~Hz}$ a $440 \mathrm{~Hz}$, dimensioni $247 \times 93 \times 28 \mathrm{~cm}$.

Dono al Teatro La Fenice degli Amici della Fenice, gennaio 1998.

e-mail:info@amicifenice.it www.amicifenice.it

\section{Incontro con l'opera}

lunedì 5 novembre 2012 ore 18.00 Sergio CofFerati

\section{Otello}

venerdì 9 novembre 2012 ore 18.00

Giorgio Pestelli

Tristan und Isolde

lunedì 14 gennaio 2013 ore 18.00

Giorgio Pestelli

I masnadieri

venerdì 8 marzo 2013 ore 18.00

Matteo Marazzi

Věc Makropulos

lunedì 11 marzo 2013 ore 18.00

GIOVANNI BIETTI

La cambiale di matrimonio

venerdì 26 aprile 2013 ore 18.00

LuCA Mosca

Don Giovanni

lunedì 29 aprile 2013 ore 18.00

LuCA Mosca

Così fan tutte

mercoledì 8 maggio 2013 ore 18.00

LUCA MOSCA

Le nozze di Figaro

venerdì 14 giugno 2013 ore 18.00

Michele DaLl'Ongaro

Madama Butterfly

venerdì 5 luglio 2013 ore 18.00

Massimo Contiero

Otello

lunedì 30 settembre 2013 ore 18.00

PaOlo Furlani

Aspern

\section{Incontro con il balletto}

lunedì 17 dicembre 2012 ore 18.00

Marinella GUATTERINI

Lo schiaccianoci tutti gli incontri avranno luogo presso il Teatro La Fenice - Sale Apollinee 


\section{$y$}

Fondazione TeATro La FenICE

DI VENEZIA

\section{Incontri con}

mercoledî 17 ottobre 2012

ore 17.30

mercoledí 5 dicembre 2012

ore 17.30

mercoledì 20 febbraio 2013 ore 17.30

mercoledì 27 febbraio 2013 ore 17.30

mercoledì 20 marzo 2013
ore 17.30

data da definire

lunedì 6 maggio 2013

ore 17.30

mercoledî 15 maggio 2013

ore 17.30

mercoledì 29 maggio 2013 ore 17.30

mercoledì 5 giugno 2013 ore 17.30

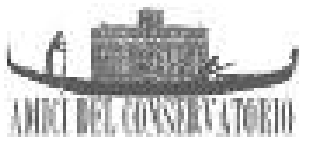

CONSERVATORIO DI VENEZIA

\section{la stagione sinfonica}

Conferenze introduttive alla Stagione sinfonica 2012-2013

del Teatro La Fenice

relatore Marco Peretti

concerti diretti da Yuri Temirkanov (22 ottobre)

Diego Matheuz (7 e 9 dicembre)

musiche di Čajkovskij, Musorgskij

relatore Franco Rossi

concerto diretto da Stefano Montanari (13 e 14 dicembre)

musiche dal repertorio marciano

relatore Francesco Erle

concerto diretto da Diego Matheuz (22 e 23 febbraio)

musiche di Mozart, Čajkovskij

relatore Maria Girardi

concerto diretto da Diego Matheuz (1 e 2 marzo)

musiche di Cascioli, Čajkovskij

\section{relatore Corrado Pasquotti}

concerto diretto da Gabriele Ferro (22 e 24 marzo)

musiche di Micheli, Stravinskij, Prokof'ev

relatore Giovanni Battista Rigon

concerto diretto da Claudio Scimone (26 e 28 aprile)

musiche di Mozart

relatore Michael Summers

concerto diretto da Stefano Montanari (8 e 9 maggio)

musiche di Costanza, Mozart

relatore Stefania Lucchetti

concerti diretti da Rinaldo Alessandrini (16 e 17 maggio; 24 e 26 maggio)

musiche di Alessandretti, Mozart

relatore Paolo Zavagna

concerto diretto da Dmitrij Kitajenko (1 giugno)

musiche di Čajkovskij, Stravinskij

\section{relatore Massimo Contiero}

concerti diretti da Diego Matheuz (7 e 8 giugno)

Myung-Whun Chung (19 luglio)

musiche di Prokof'ev, Čajkovskij, Verdi

Tutti gli incontri avranno luogo presso la Sala Concerti

del Conservatorio di Musica Benedetto Marcello di Venezia. 
(1)

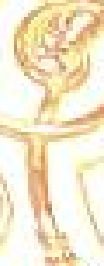

(2)

S.

$6{ }^{2}$

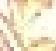

$6^{2} \mathrm{es}$

320

Sit.

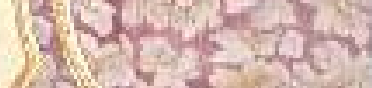

20 .

4e

)

(1) 15

2) 5 is $=53$

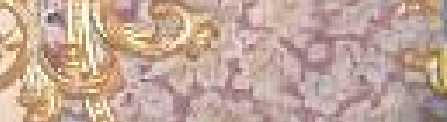

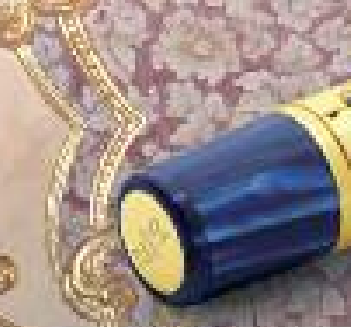

18

ton

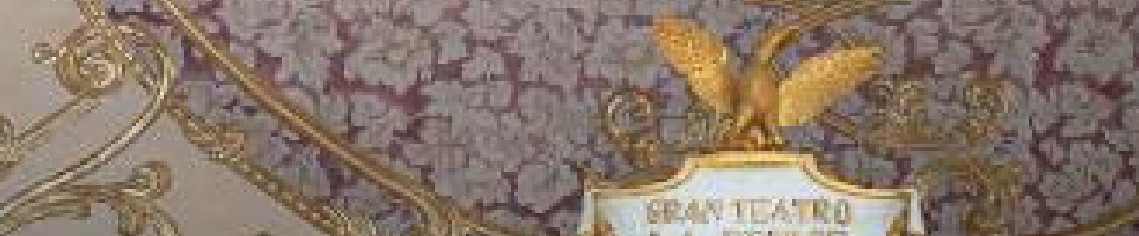

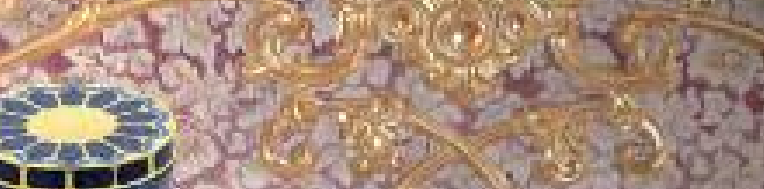

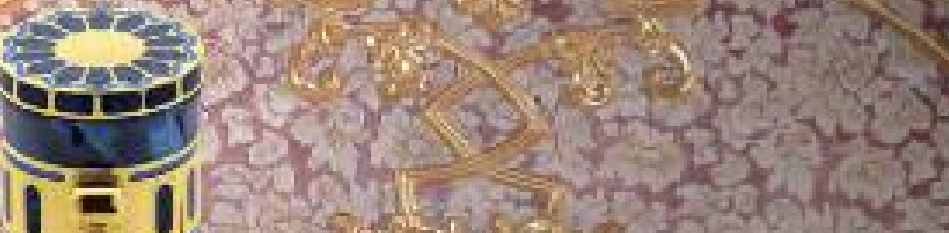



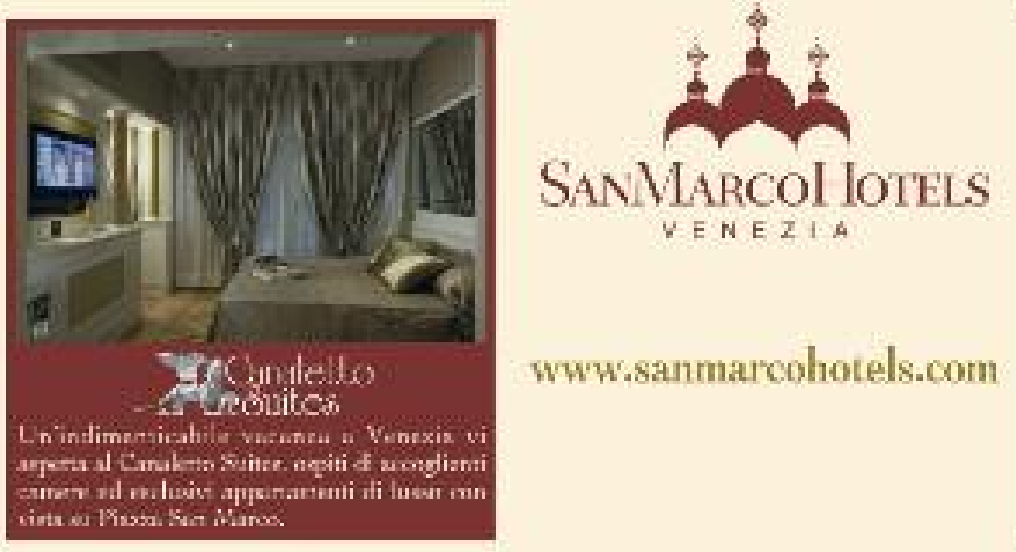

wwr.sanmarcohotels.com

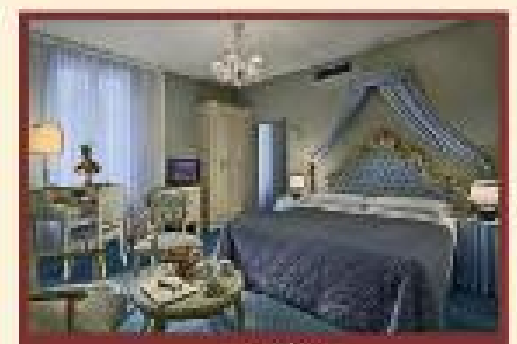

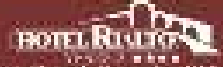

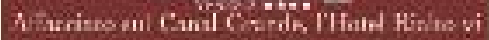

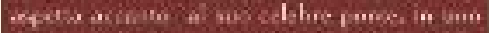
degh uncoli rou mugentic e stramolinari dala

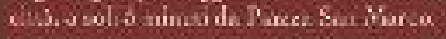

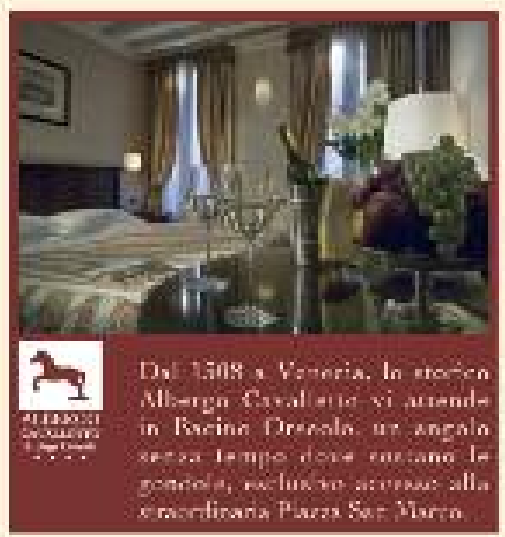

Luxury Apsurmants Belkvut Suites

Sun Mism 285.50124 Vonexis

Tid. +30041 wes 212 is

Fas + 79641 .328 7688

inforitalkvosauilosin

Suices Tueve dell'Orulogie

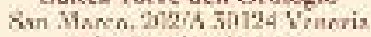

Tit. + 3041 211 ol 11

$F_{35}+396415287589$

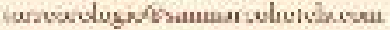

\section{Dother}

Sun Mlamo Palare Suitro

Suin Mience 47530124 Vencriu

T. +3904124.451

$16+30041213436$

esnmarropsiarmptonmsmotenelecorm
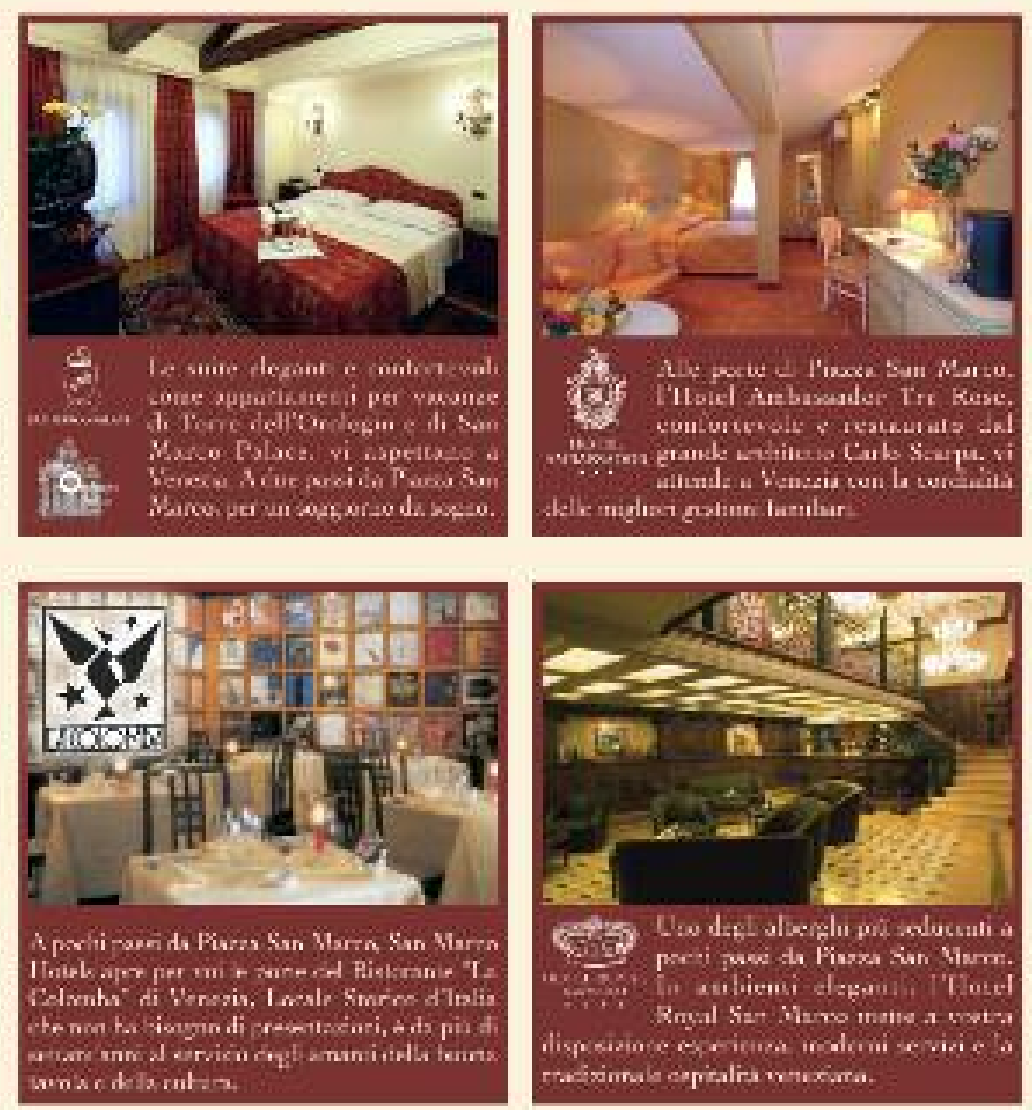

5 superior

Hetel Crvalletio \& Thope Orenolo

Sin Marra, 1100.70124 Verrocis Tel. + 39 latt fiso nes 5:

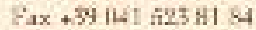

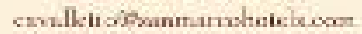

Hetel Kisilw

Sun Marws, GIf9 Yot24 Veruxia

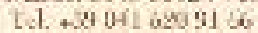

$8 x+3904162589 \leq 8$

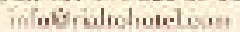

Hotel Regal Saz Marco

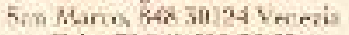

r,l. +31 0415287665

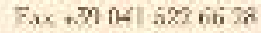

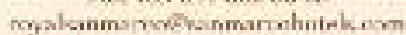
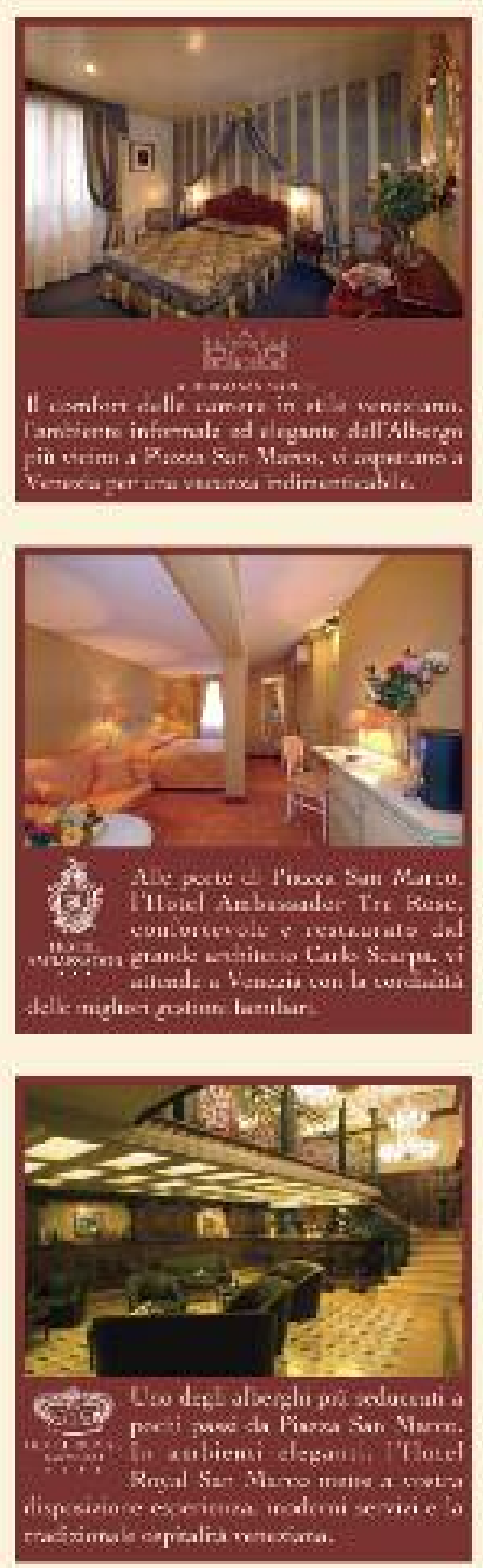

Comtnor

Altwergn San Marm

Sin Muren, 877,30124 Venrxix

Tiel. -3y o41 501 -277

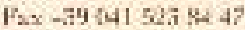

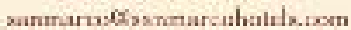

Huisl Ambasadere Tie Kuse

Sin. Mutcu. Yutir 30125 Vemsis li.l. -39 ch1 2222490

Fex-\$9 3415222128

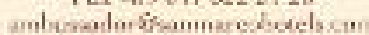

Ristorante La Colombe

Sin Murco, Jens 3124 Venexid

TeL. -39 (4) 5191175

Fix $-39<415721<08$

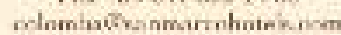




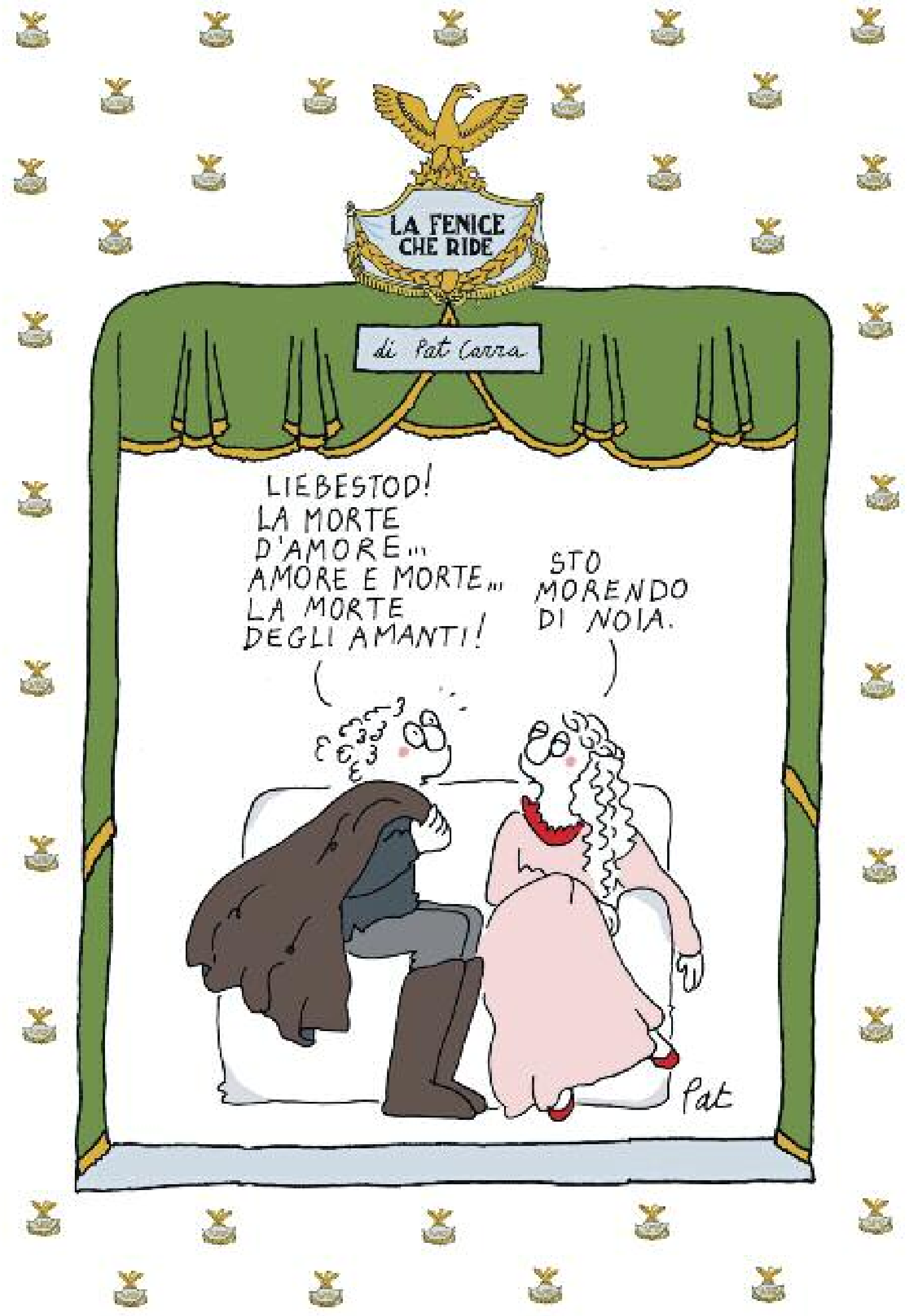




\title{
La vita è fatta di momenti importanti. tu quale stai vivendo?
}

\author{
www inaassitalia.it \\ consulta il sito per scoprire \\ le soluzioni assicurative \\ pensate da INA ASSITALIA \\ per ogni momento importante \\ della tua vita \\ e di quella del tuoi cari.
}




\section{PERMASTEELISA GROUP}

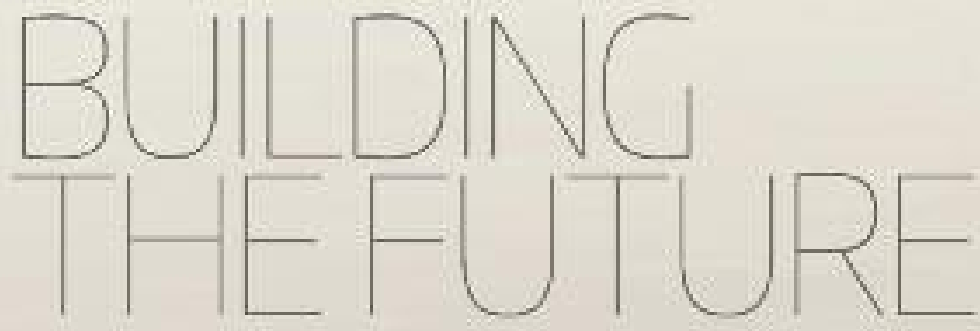

Permasteelisa Group cot,abora con prestigos istituti e

Un wersita nte tazionel per sviluppare ricerca, innoveziore a sperimentazionespalicace allin retitelfuta

II Grapan Pormantachisa nesera a livela mondialo of sattope dolia progetez ore, produzone ac nect.azziene di ivveloeri acen lekenici, feciate

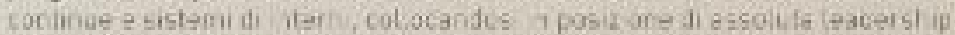

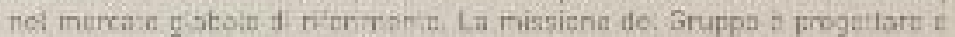

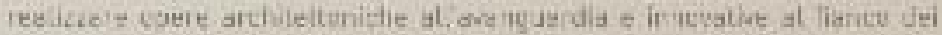
grandi nomi delarhitetura costamporanea applicando salusiorl ad

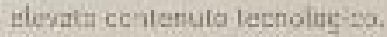

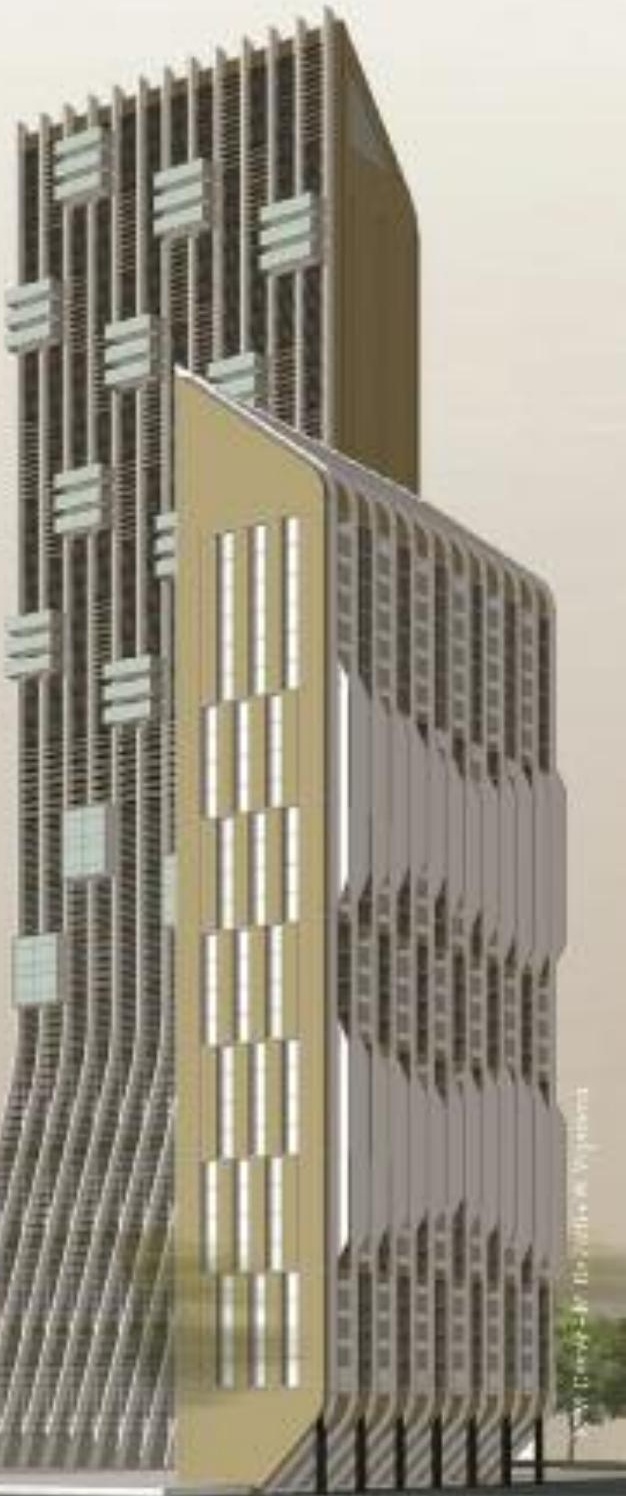

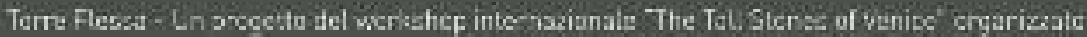

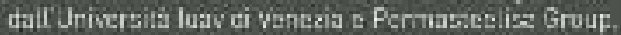

Permasteetisa S.p.A. I Headquarters / Viaie E. Mattei $21 / 23131029$ Vittorio Vaneto, Treviso www.permasteelisagroup.com 


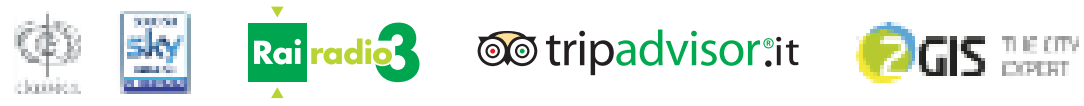
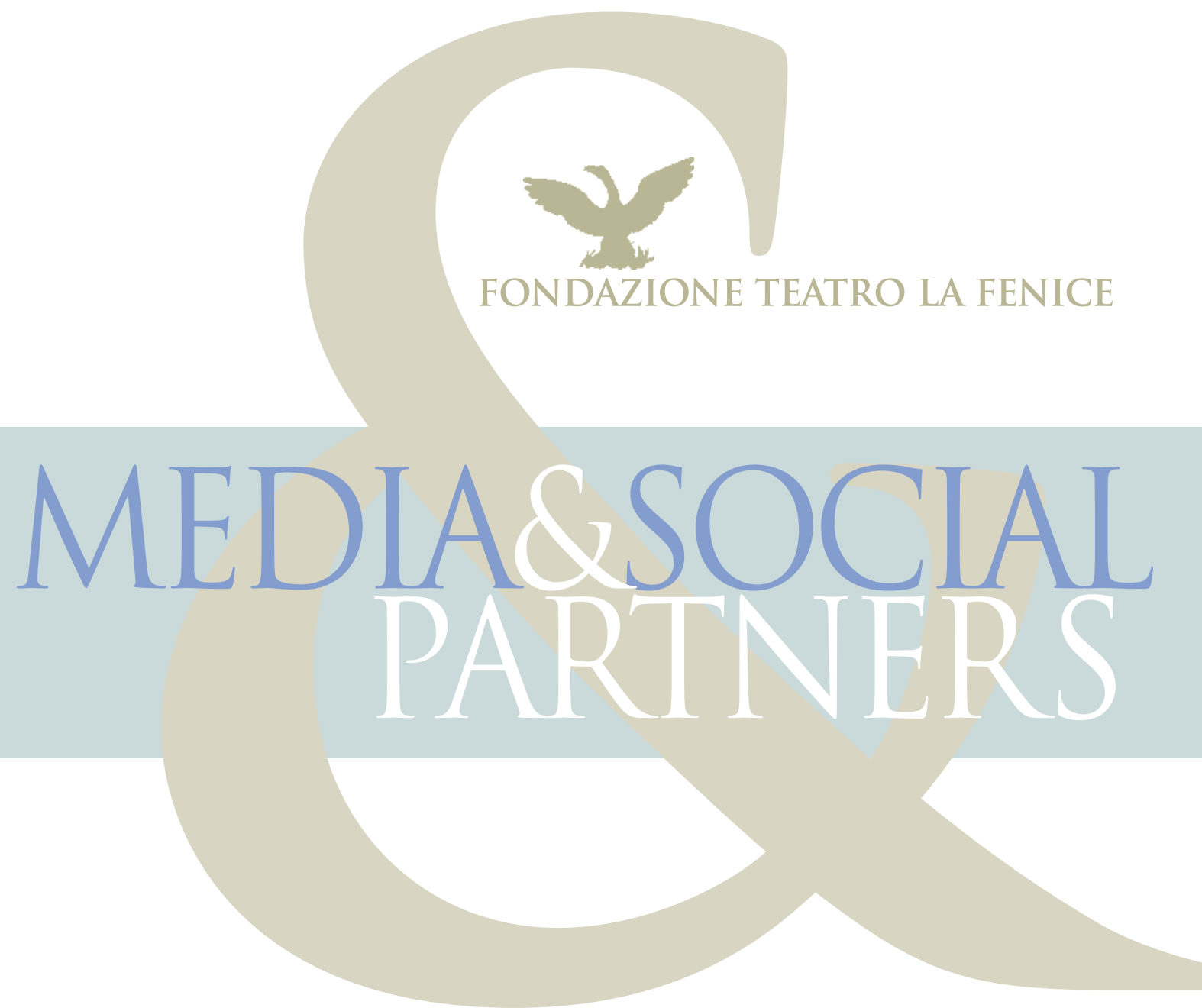

f 3 

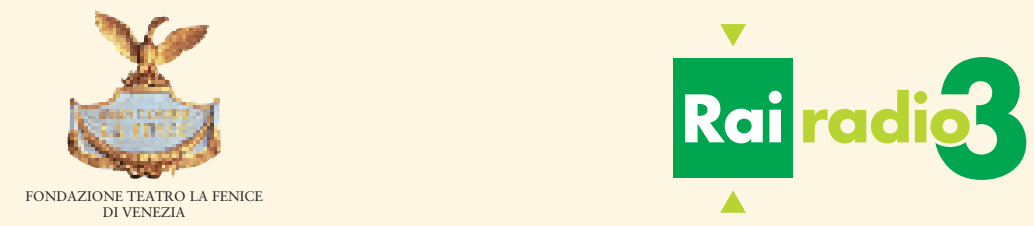

\section{Radio3 per la Fenice}

Opere della Stagione lirica 2012-2013 trasmesse dal Teatro La Fenice o dal Teatro Malibran

venerdì 16 novembre 2012 ore 19.00 diretta

Otello

domenica 20 gennaio 2013 ore 19.00 diretta Euroradio

I masnadieri

venerdì 15 marzo 2013 ore 19.00 differita

Věc Makropulos

mercoledì 2 ottobre 2013 ore 19.00 differita

Aspern

Concerti della Stagione sinfonica 2012-2013 trasmessi in differita dal

Teatro La Fenice o dal Teatro Malibran

Diego Matheuz (venerdì 5 ottobre 2012)

Yuri Temirkanov (lunedì 22 ottobre 2012)

Stefano Montanari (mercoledì 8 maggio 2013)

Rinaldo Alessandrini (giovedì 16 maggio 2013)

Dmitrij Kitajenko (sabato 1 giugno 2013) 
Fondazione Teatro La Fenice di Venezia

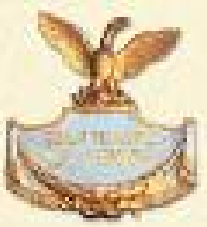




\section{Fondazione Teatro La Fenice di Venezia Albo DEI FondATORI}

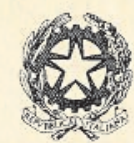

REGIONE DEL VENETO

\section{SOCI SOSTENITORI}

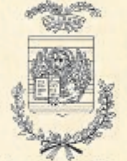

Provincia di Venezia

Banca

Popolare di Vicenza

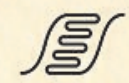

CONSORZIO VENEZIA NUOVA

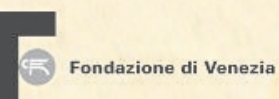

SOCI BENEMERITI

Camera di CoMarercio

INDUSTRLA ARTIGIANATO E AGRICOLTURA

VENEZIA

\section{器 GENERALI}

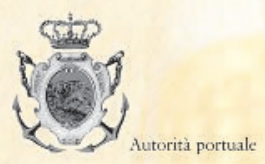

AIVE

group
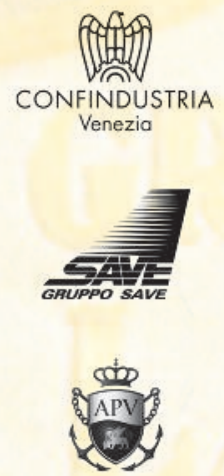

APV INVESTIMENTI

\section{superjet}




\section{Fondazione Teatro La Fenice di Venezia}

\section{CONSIGLIO DI AMMINISTRAZIONE}

Giorgio Orsoni

presidente

Giorgio Brunetti

vicepresidente

Marco Cappelletto

Fabio Cerchiai

Cristiano Chiarot

Achille Rosario Grasso

Mario Rigo

Luigino Rossi

Francesca Zaccariotto

Gianni Zonin

consiglieri

sourintendente

Cristiano Chiarot

direttore artistico

Fortunato Ortombina

direttore principale

Diego Matheuz

\section{COLLEGIO DEI REVISORI DEI CONTI}

Anna Maria Ustino, presidente

Annalisa Andreetta

Giampietro Brunello

Andreina Zelli, supplente

SOCIETÀ DI REVISIONE

PricewaterhouseCoopers S.p.A. 


\section{Fondazione Teatro La Fenice di Venezia Albo DEI FondATORI}

\section{SOCI ORDINARI}

\author{
initi \\ I.TIII \\ Fondazione Amici della Fenice
}

COMITÉ FrançAIS

POUR LA SAUVEGARDE DE VENISE

\section{CASINOST
DIVENEZIA}

YMarsilio

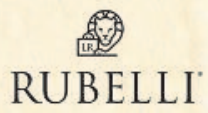

STUDIO DE POLI
l'Adige

CORAIEAE DEULALTO ADTEE CORBIEAE DEL TAENTINO
PRICEWATERHOUSECOOPERS 圈

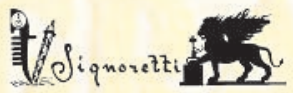




\section{TRISTAN UND ISOLDE \\ (Tristano e Isotta)}

azione in tre atti WWV 90

libretto e musica di Richard Wagner

\section{Teatro La Fenice}

domenica 18 novembre 2012 ore 15.30 turno A venerdì 23 novembre 2012 ore 17.00 turno B domenica 25 novembre 2012 ore 15.30 turno C mercoledì 28 novembre 2012 ore 17.00 turno D sabato 1 dicembre 2012 ore 17.00 turno $\mathrm{E}$

La Fenice prima dell’Opera 2012-2013 2 


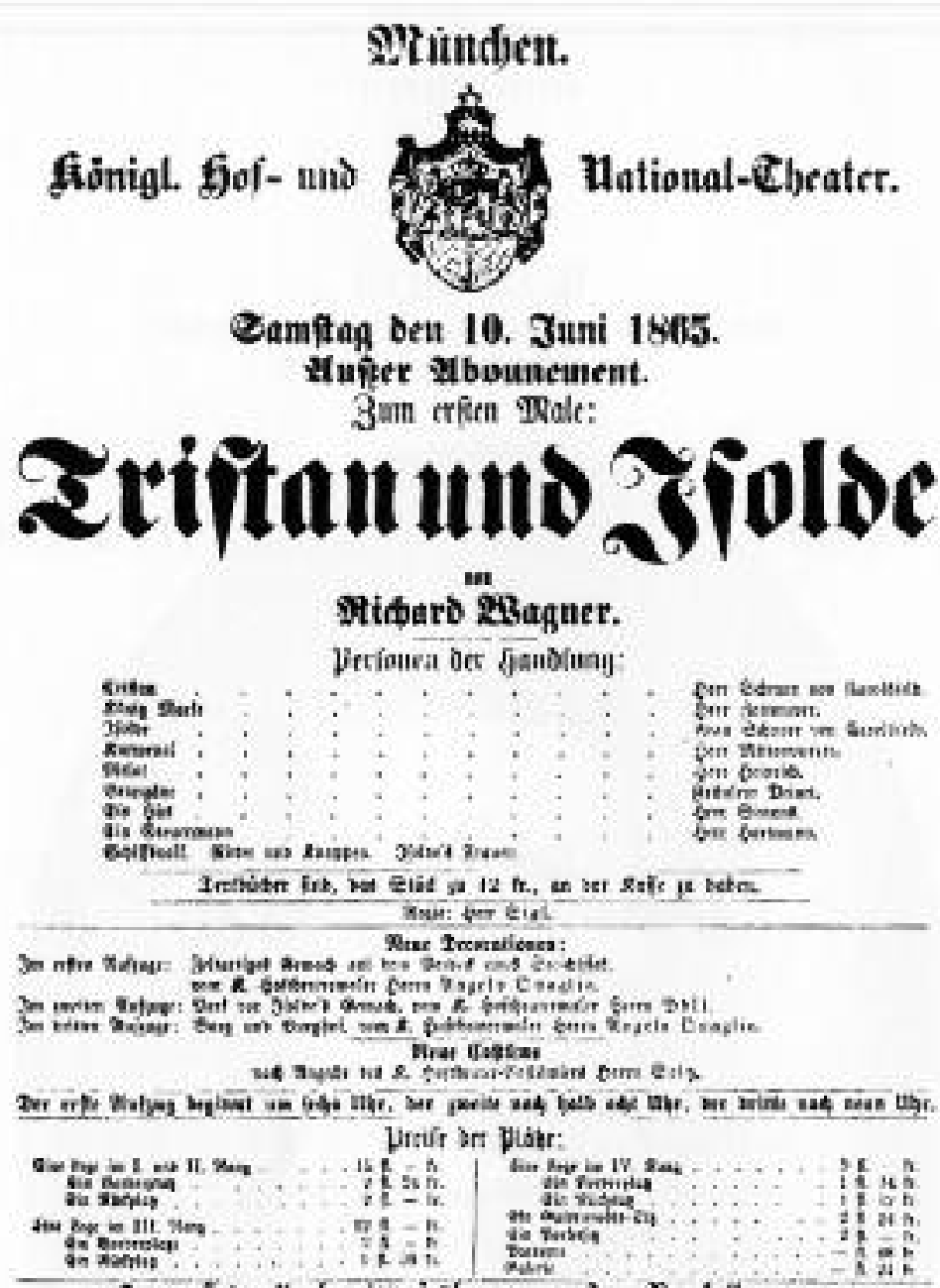

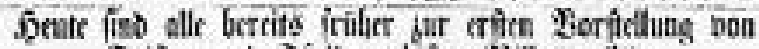
zripan unb Gioloe celoften Billetes giltig.

IIrfö̀n un jechs Ubr, (Fibe nach jebu thlor.

Fer fore cyinfrite tif obus atle Gitonahone anterkoben

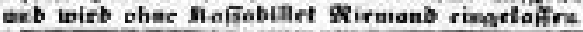

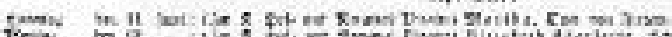

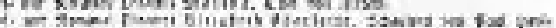

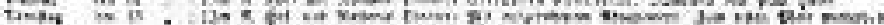

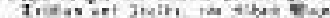

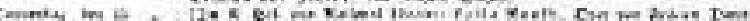

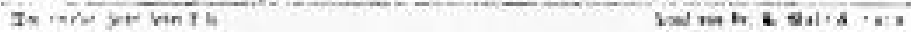

Locandina della prima rappresentazione di Tristan und Isolde al Teatro Nazionale di Corte di Monaco, 1865. 
La Fenice prima dell'Opera 2012-2013 2

Sommario

7 La locandina

9 'Tristanismi' fin de siècle

di Michele Girardi

13 Virgilio Bernardoni

«Ewig, ewig ein»: interiorizzazione poetica

e azione musicale in Tristan und Isolde

31 Guido Paduano

La storia d'amore (parafrasi di Tristano e Isotta)

51 Tristan und Isolde: libretto e guida all'opera a cura di Riccardo Pecci

155 Tristan und Isolde in breve a cura di Gianni Ruffin

157 Argomento - Argument - Synopsis - Handlung

165 Riccardo Pecci

Bibliografia

171 Dall'archivio storico del Teatro La Fenice

La prima volta alla Fenice di Antonio Guarnieri a cura di Franco Rossi

178 Biografie 


\section{TEATRO LA FENICE DI VENEZIA \\ STAGIONE 2012-2013 \\ DOPPIA INAUGURAZIONE \\ 16 NOVEMBRE 2012 - OTELLO \\ 18 NOVEMBRE 2012 - TRISTAN UND ISOLDE}

si ringraziano per lo speciale contributo

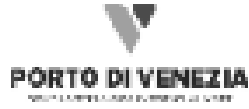

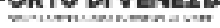

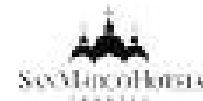

SOPABROKER
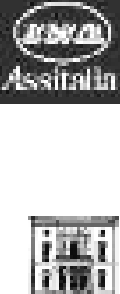

Fisulaxicuc Amiei dklla Fonis:

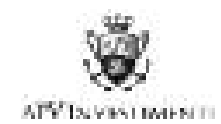

CASINOT:S
DIVENEZIA

POクTE

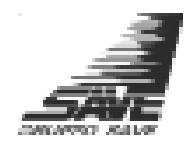

The Venice International Foundation

FREUNDESKREIS DES

TEATRO LA FENICE 
Gérard Auguier

Massimo Benetello

Barbara e Alberto Berlingieri

Alessandra Biasutti

Mariuccia e Marco Bortoli

Paolo Cantarella

Lino Cazzavillan

Fabio Cerchiai

Franca e Piergiorgio Coin

Iaia e Vittorio Coin

Matteo Corvino

Lorenza de' Medici

Barbara e Lodovico di Valmarana

Alessandro Favaretto Rubelli

Tinetta Gardella

Paolo Jucker

Gaetano Maccaferri

Carla Macola Bonsembiante

Chantal e Alain Mérieux

Claude Paluc

Irene Pellegrini Falck

Giovanna Pitteri Larghieri

Vittorio Radice

Agnès e Louis Schweitzer

Alessandra Talarico

Andrea Tomat

Massimo Zanetti

Lucia Zavagli Ricciardelli

Jérôme-François Zieseniss 


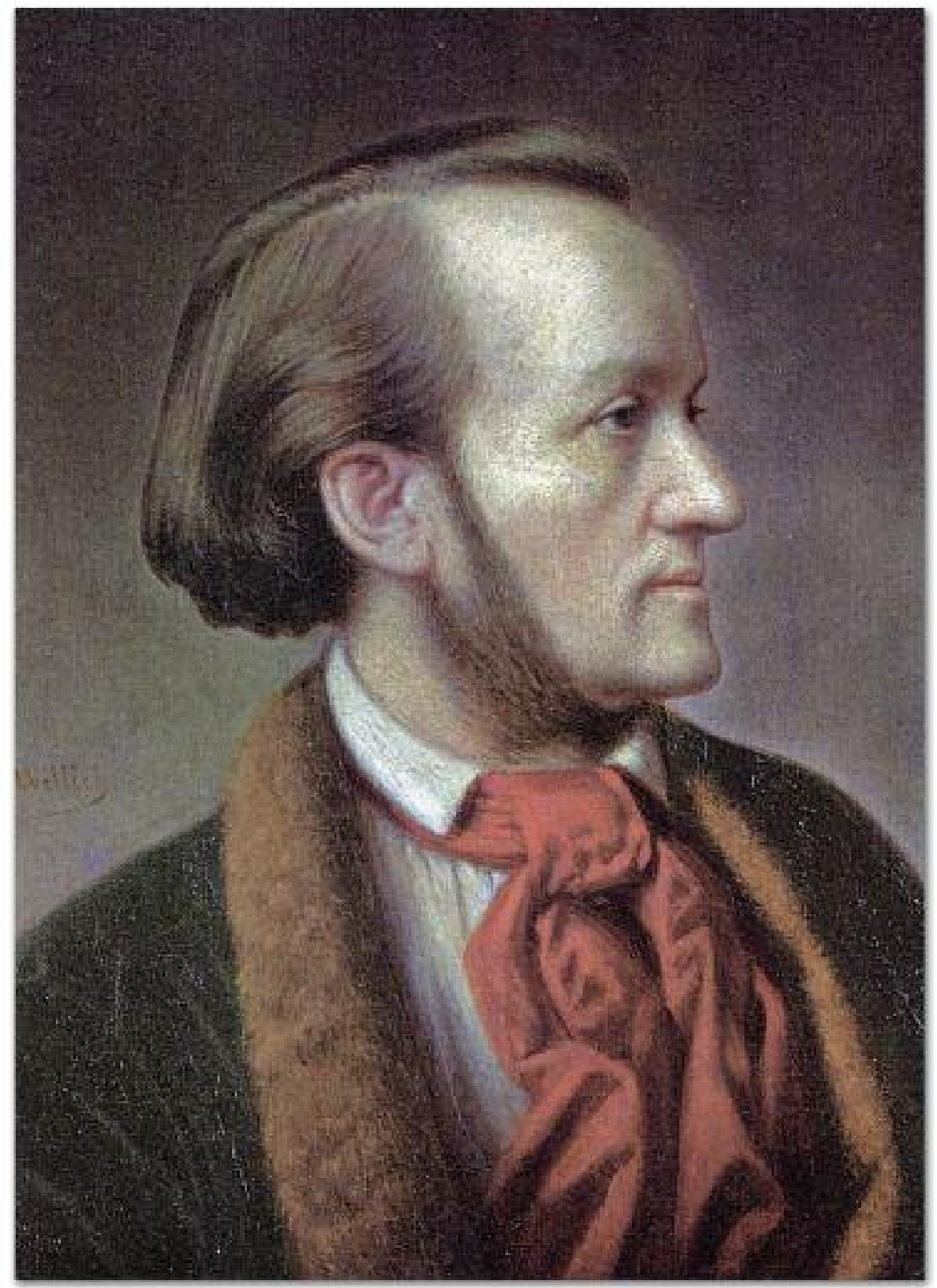

Cäsar Willich (1825-1886), Richard Wagner (1862). Olio su tela. Musikbibliothek der Stadt Leipzig. 


\section{TRISTAN UND ISOLDE}

(TRISTANO E ISOTTA)

azione in tre atti WWV 90

libretto e musica di

Richard Wagner

dal romanzo in versi Tristan di Gottfried von Straßburg

prima rappresentazione assoluta:

Monaco di Baviera, Königliches Hof- und Nationaltheater, 10 giugno 1865

edizione critica a cura di Isolde Vetter ed Egon Voss

editore proprietario Schott Music, Mainz

rappresentante per l'Italia Sugarmusic, Milano

personaggi ed interpreti

Tristan Ian Storey

Re Marke Attila Jun

Isolde Brigitte Pinter

Kurwenal Richard Paul Fink

Melot Marcello Nardis

Brangäne Tuija Knihtilä

Un pastore Mirko Guadagnini

Un pilota Armando Gabba

Un giovane marinaio Gian Luca Pasolini

maestro concertatore e direttore

Myung-Whun Chung

regia Paul Curran

scene e costumi Robert Innes Hopkins

light designer David Jacques

\section{Orchestra e Coro del Teatro La Fenice}

maestro del Coro Claudio Marino Moretti

in lingua originale con sopratitoli in italiano e in inglese

nuovo allestimento Fondazione Teatro La Fenice

nel bicentenario della nascita di Richard Wagner

spettacolo sostenuto dal

FREUNDESKREIS DES

TEATRO LA FENICE 


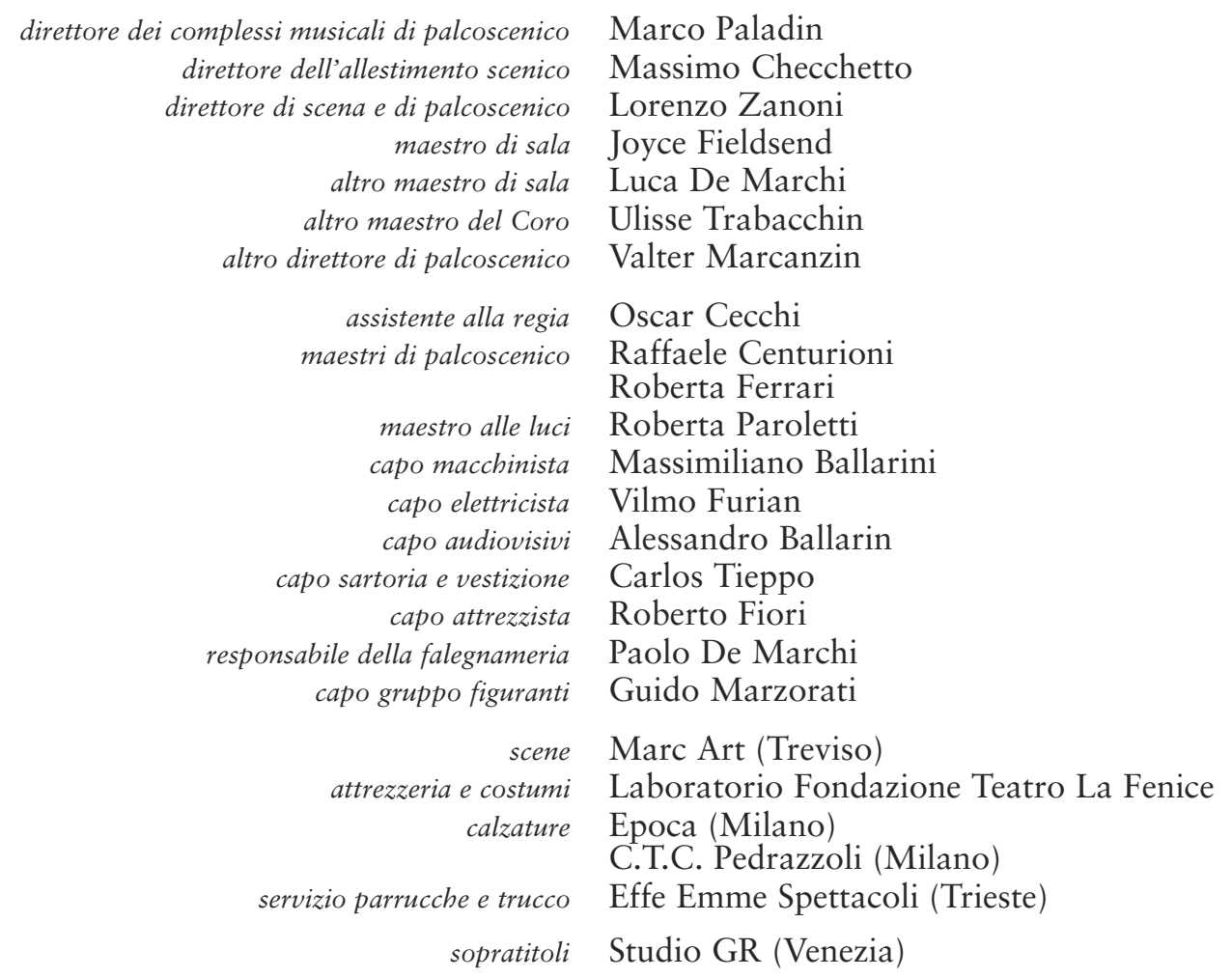




\section{‘Tristanismi’ fin de siècle}

Il giornalista Guido Marotti ci narra un episodio dell'ultimo «inverno» (era in realtà «una sera d'ottobre» ${ }^{1}$ ) della vita di Giacomo Puccini:

Una tarda sera dell'inverno 1924, Puccini ed io, soli nella sua saletta di musica, là nella villa viareggina. Turandot taceva: il Maestro era intento ad altro. La tastiera del pianoforte, aperta come al solito, mi tentò; e non so perché mi venne fatto di piantare le dita su quell'accordo di settima diminuita-alterata, $\mathrm{Fa}_{2}-\mathrm{Si}_{2}-\mathrm{Re}_{3}-\mathrm{Sol}_{3}$ col quale, dopo il $\mathrm{La}_{2}-\mathrm{Fa}_{3}-\mathrm{Mi}_{3}-\mathrm{Re} \#_{3}$ [dei violoncelli], ha inizio il Tristano. Puccini alzò il capo e mi guardò sorpreso...: «O questa, poi, come t'è saltata in mente?». Non s'era ancor spenta la vibrazione di quelle note, ch'egli disse: «E pensare che, proprio questo accordo, è la chiave magica con cui s'apre la porta della musica contemporanea!». Poi si levò dalla poltrona e, tratto da un armadio lo spartito del Tristano, venne al pianoforte e si dette con lena e trasporto a suonarne il «preludio». Però, ad un tratto, troncò: «Basta, basta con questo tormento cromatico che mi dà l'agitazione e mi disturba: è musica terribile, pericolosa, come se entrassi fra le spire d'un grande serpente: occorre non farcisi prendere, altrimenti se ne rimane le vittime! [...] Noi siamo veramente dei mandolinisti, a paragone di questo ossessivo colosso!... Capisco Debussy, capisco Ravel, Stravinskij e tanti altri che ne sono fuggiti lontani».2

Puccini, più che un mandolinista, fu uno dei più fini e personali interpreti della lezione wagneriana sulla scena europea, e la sua reazione al preludio di Tristan und Isolde è emblematica: nei decenni successivi alla première di questo capolavoro (1865), centinaia di mani avrebbero 'piantato le dita' sul primo accordo della partitura, «chiave magica» per aprire le porte della modernità, e molti compositori di punta l'avrebbero ripreso - nemmeno Debussy vi si sarebbe sottratto nel Children's Corner (1908), pur se in chiave affettuosamente ironica (Golliwogg's Cakewalk, bb. 61-63, e segg.).

Non era stato per nulla beffardo, fra i tanti musicisti francesi fin de siècle che citarono l'accordo (e/o fecero riferimento alla tavolozza armonica e ai topoi drammatici di Wagner), Jules Massenet. Egli utilizzò innumerevoli volte la 'settima' magica al servizio del dramma, potenziandone lo sviluppo e l'impatto emotivo, da Hérodiade (1882) passando per Esclarmonde (1889) fino a Werther (1892). Nel capolavoro trat-

\footnotetext{
1 Puccini sarebbe morto il 29 novembre del 1924: Marotti aveva fornito una data più verosimile raccontando lo stesso episodio nel suo Giacomo Puccini intimo, Firenze, Vallecchi, 1942, pp. 203-204; in ogni caso le testimonianze di Marotti sono intrise di retorica e faziosità e vanno sempre vagliate con cura.

2 GUIDO MAROTTI, Incontri e colloqui col Maestro, «L'approdo musicale», II/6, 1959, pp. 53-71: 63-64.
} 
to da Goethe, dopo che Charlotte ha lasciato la stanza del protagonista nel tragico finale terzo, il tenore tenta di riscuotersi per un attimo dall'angoscia, si guarda intorno, è solo e tormentato: su di lui piomba il peso della propria disfatta amorosa che trova in quell'accordo

III, 210

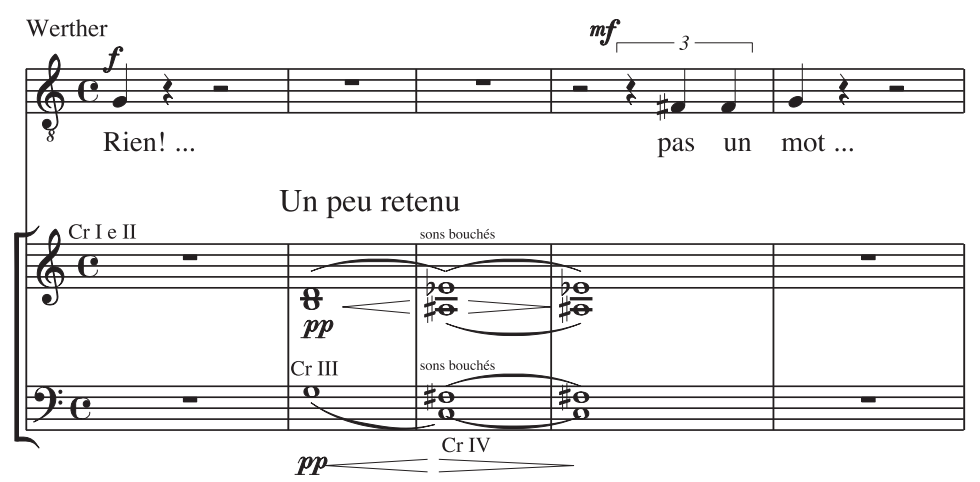

la medesima premessa ineluttabile alla sorte mortale di Tristan, e che qui vivifica una fitta rete di rapporti intertestuali.

Il nostro viaggio nella musica fin de siècle (e oltre) potrebbe continuare a lungo: nel contesto dell'enorme letturatura secondaria dedicata al musicista di Lipsia, il tema della ricezione musicale e drammatica di Tristan und Isolde è affascinante e fra i più suscettibili di sviluppo, ${ }^{3}$ ma questo esempio mi pare già significativo dell'ampiezza d'interessi sollecitati da quell'accordo fin dalla sua prima comparsa. L'argomento viene ripreso da Riccardo Pecci nelle pagine di questo volume, in una stimolante guida all'opera ricca di spunti critici nuovi che mette la partitura del capolavoro wagneriano sotto la lente d'ingrandimento, e propone il testo del libretto, secondo i moderni orientamenti, in una versione più vicina a quella che Wagner stesso avrebbe probabilmente auspicato. La sezione saggistica, come quella di Otello, viene dal programma di sala pubblicato dalla Fenice nel 2002. Se l'ispirata 'parafrasi' di Guido Paduano (in realtà una potente chiave ermeneutica della drammaturgia di Tristan) riappare con pochi ritocchi, il saggio iniziale di Virgilio Bernardoni esce in una nuova versione, aggiornata dall'autore.

\section{Michele Girardi}

\footnotetext{
3 Si legga, sin d'ora, una lunga lista di autori e titoli che arriva sin quasi ai giorni nostri, proposta e commentata con intelligenza musicale e drammatica da STEVEN HuEBNER, «Tristan»'s Traces, in Richard Wagner: «Tristan und Isolde», a cura di Arthur Groos, Cambridge, Cambridge University Press, 2011, pp. 142-166, 195-198. Sui rapporti fra l'estetica wagneriana e l'intertestualità legata al Tristanakkord, si veda MichELE GIRARDI, Massenet à Puccini, "Heureux de votre grand triomphe»: un maitre français pour un génie italien, di prossima pubblicazione negli atti del convegno internazionale Massenet aujourd'hui: héritage et postérité, Saint-Étienne, 25-26 ottobre 2012.
} 

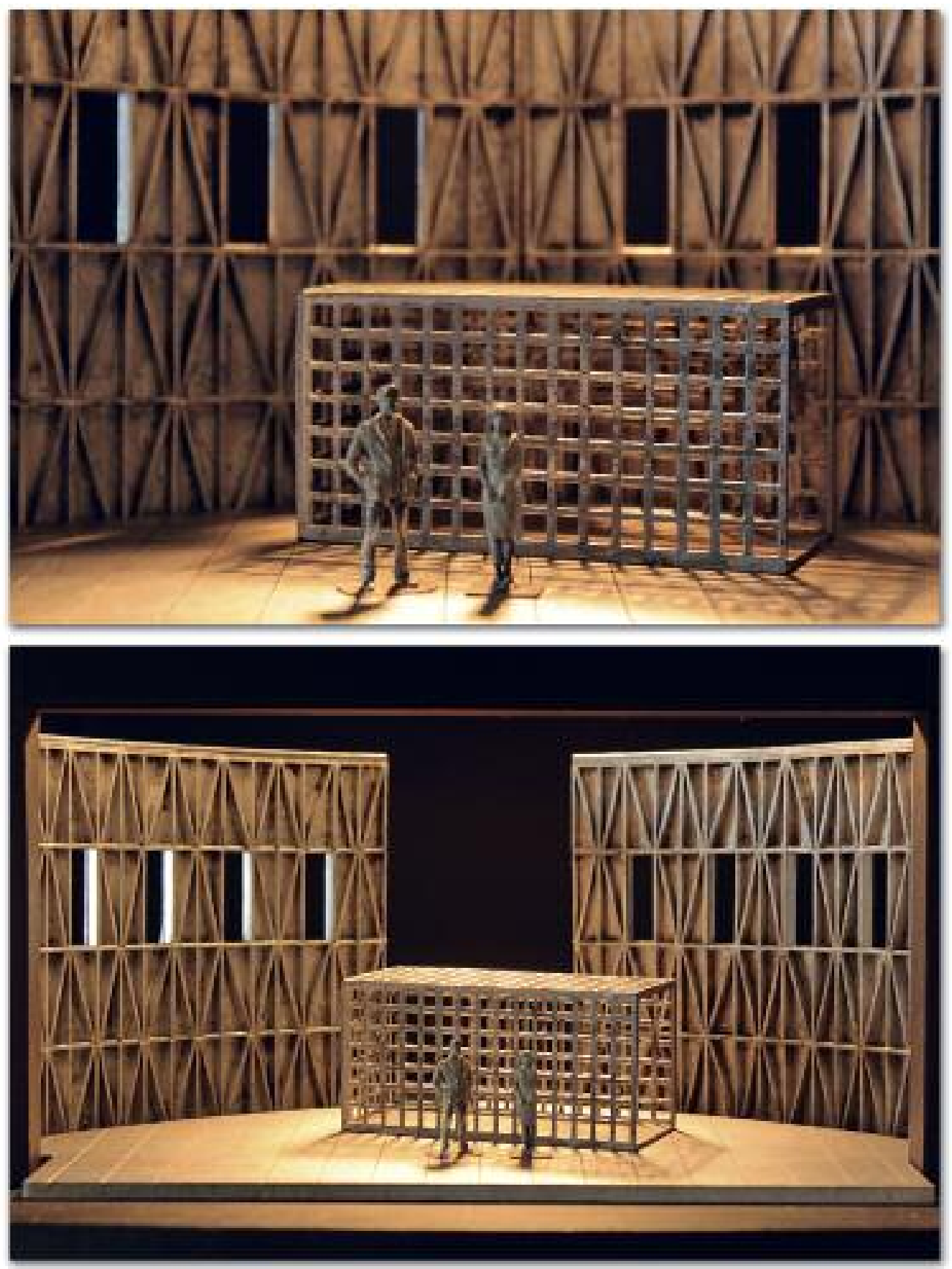

Robert Innes Hopkins, modellini scenici (I) per Tristan und Isolde al Teatro La Fenice di Venezia, 2012; regia di Paul Curran, costumi di Robert Innes Hopkins. 

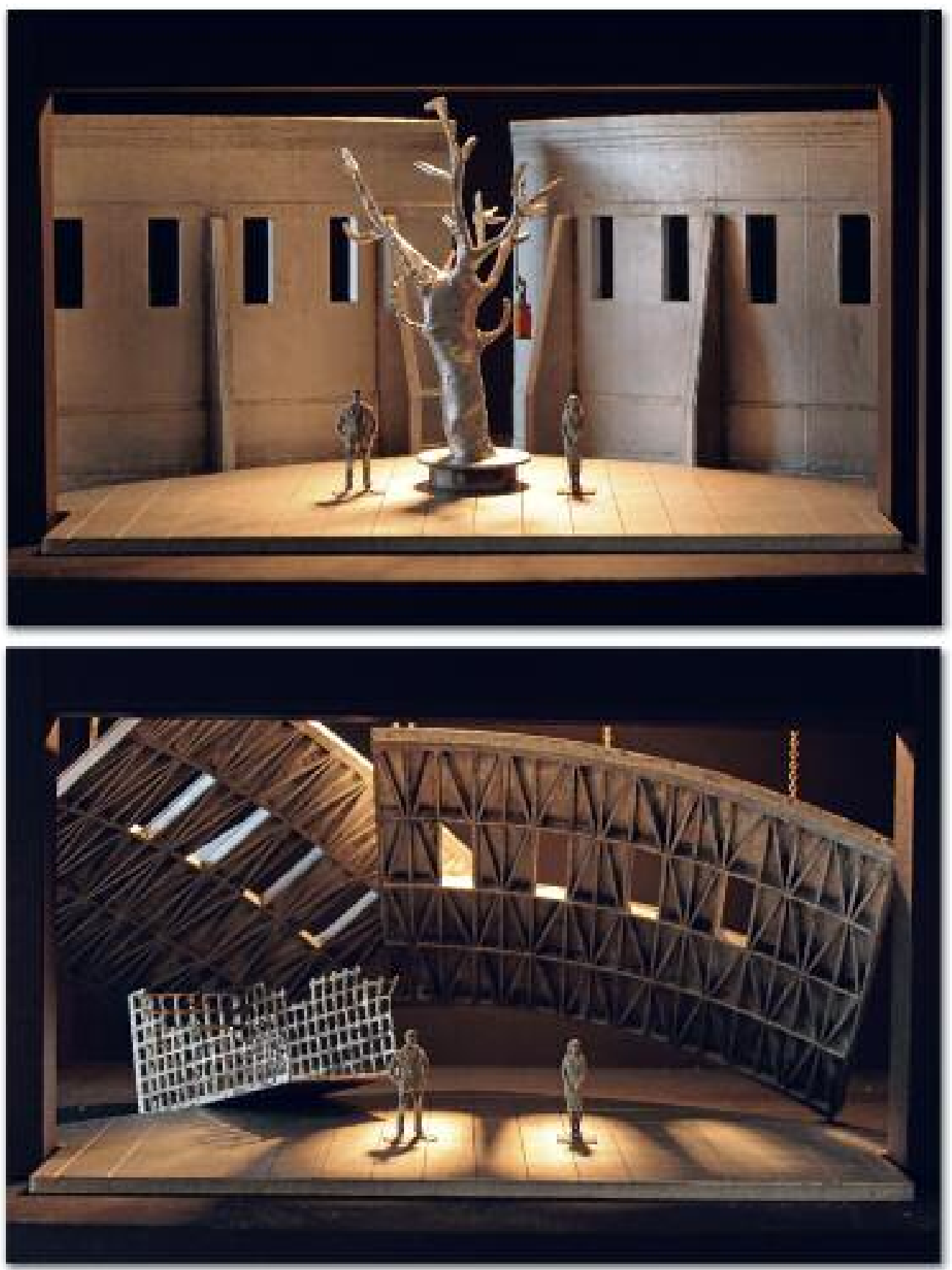

Robert Innes Hopkins, modellini scenici (II; III) per Tristan und Isolde al Teatro La Fenice di Venezia, 2012; regia di Paul Curran, costumi di Robert Innes Hopkins. 


\section{Virgilio Bernardoni \\ «Ewig, ewig ein»: interiorizzazione poetica e azione musicale in Tristan und Isolde}

\section{Un dramma duale}

È insita nella materia di Tristan und Isolde una fondamentale radicalizzazione degli opposti: il senso dell'azione si compendia nella negazione pertinace della volontà di vivere e nell'affermazione parimenti persistente della valenza eterna della volontà di amare. Su questo dualismo, e sul significato anti-sociale di un valore che s'attesta al di là delle sfere del diritto e della morale, lo stesso Wagner concentra l'attenzione nel commento al Vorspiel $z u$ «Tristan und Isolde» redatto a Parigi nel 1859:

Il fido vassallo aveva richiesto a nome del proprio re la mano di Isotta, ch'egli stesso, senza in cuor suo riconoscerlo, amava: e Isotta, che lo seguitava come sposa promessa del di lui re, era a sua volta irresistibilmente soggiogata dal pretendente per procura. Ma la dea d'amore, ingelosita, volle vendicare l'oltraggio, pravamente inflitto ai suoi diritti: mediante un equivoco ingegnoso, essa induce la giovane coppia a delibare il filtro che, secondo l'uso di quei tempi, la premurosa madre della sposa aveva distillato per infiammare d'amore il matrimonio regale, dettato dalla mera politica; nell'ardore amoroso che il filtro ha acceso in loro, i due giovani riconoscono la passione che indissolubilmente li lega. Si scatena il desiderio, la bramosia, la voluttà, la pena dell'amore: il mondo, la forza, la gloria, il fasto, la cavalleria, la fedeltà, l'amicizia, tutto si dilegua come un sogno evanescente: non sopravvive altro che il desiderio, lo struggimento, la brama insaziabile e sempre più impetuosa - lo spasimo, assetato e inestinguibile, unica estrema redenzione - la morte, la consunzione, la dissoluzione, il sonno perpetuo! ${ }^{1}$

In questo profilo, in cui traccia le linee programmatiche del dramma, Wagner dedica minima attenzione agli eventi tangibili, i quali sono riconducibili essenzialmente alle premesse della vicenda (l'attività di procuratore matrimoniale svolta da Tristano per conto di re Marke) e all'episodio della delibazione del filtro magico, e fa passare totalmente sotto silenzio gli eventi dell'atto terzo (la fuga di Tristano ferito nella nativa Kareol, l'attesa spasmodica di Isotta, il perdono di re Marke). Per contro, egli rileva in mo-

${ }^{1}$ Richard WaGner, Vorspiel zu «Tristan und Isolde», traduzione parziale di Lorenzo Bianconi, in CARL DAHLHAUs, I drammi musicali di Richard Wagner [Richard Wagners Musikdramen, 1971], Venezia, Marsilio, 1984, pp. 65-66. Wagner allegò questo documento, redatto per il pubblico parigino della versione da concerto del Preludio e Morte d'Isotta, alla lettera a Mathilde Wesendonck del 19 dicembre 1859 (facsimile in Richard Wagner an Mathilde Wesendonk. Tagebuchblätter und Briefe 1853-1871, a cura di Wolfgang Golther, Berlin, Verlag von Alexander Duncker, 1904, Handschrift-Nachbildung zu Seite 202, f.t.). 
do particolare gli agenti del processo di redenzione - la bramosia della felicità nella morte, nella consunzione, nella dissoluzione, nel sonno perpetuo - che s'irradiano dagli 'eventi' riferiti all'interiorità di Tristano e Isotta, quali sono rappresentati in tutte le loro implicazioni nella scena del filtro magico (I.5), nel duetto della notte d'amore (II.2) e nel canto della trasfigurazione di Isotta (III.3).

Con singolare e evidente parallelismo, i tre atti di Tristan und Isolde sono costruiti sulla transizione dagli eventi tangibili della vicenda agli eventi soggettivi dell'interiorità. Si presentano perciò come un triplice itinerario verso un'unica verità, che trascende il mondo fenomenico per svelarne una dimensione più profonda. Ciascun atto, infatti, inizia con un effetto sonoro che proviene da una fonte invisibile, ma reale (il canto senza accompagnamento del giovane marinaio nel primo, la fanfara di caccia nel secondo, la 'vecchia aria' del pastore di Cornovaglia nel terzo) e, attraverso una serie di azioni rese visibili sulla scena, va a focalizzarsi sulle azioni interiori che riguardano i due protagonisti. Ciascun atto termina con la conferma del loro particolare concetto di felicità, ribadito ogni volta nei medesimi termini lessicali: «höchste Liebeslust» e «höchste Lust», che Guido Manacorda traduce in italiano con «altissima gioia d'amore» - o anche "suprema voluttà d'amore» - e con «suprema letizia». Arthur Groos ha acutamente rilevato come l'ambiguità lessicale insita nei termini Lust e Liebeslust diventi il punto focale delle emozioni dei protagonisti e ne esprima i livelli di autocoscienza, e sia in qualche misura responsabile anche dell'irrisolta tensione interna al dramma:

Il termine tedesco Lust possiede due serie di significati: felicità (beatitudine, diletto, piacere, ecc.) e la sua anticipazione (volontà, desiderio, brama, ecc.). La medesima ambiguità caratterizza anche l'insolito composto Liebeshust [...]. È importante sottolineare come nei momenti culminanti di Tristan und Isolde l'ambiguità di Liebeslust e Lust esprima una tensione particolarmente intensa fra desiderio e realizzazione, tensione che negli atti primo e secondo è sospesa dall'arrivo di re Marke, che tutt'a un tratto trasforma la felicità in bramosia, rendendo ambigua, forse, perfino l'apparente risoluzione delle loro morti [di Tristano e Isotta] alla fine dell'atto terzo. ${ }^{2}$

La serie di opposizioni (di ambiguità) in relazione complementare che riguardano il senso dell'azione visibile (volontà di vivere versus volontà di amare) e le sue modalità narrative (da evento fenomenico a tensione interiore dei protagonisti), si collega a sua volta a una costellazione di dualismi che ne sostengono e rafforzano il significato simbolico e la funzione. Sul piano dell'azione esteriore, i precedenti delle relazioni fra Tristano e Isotta sono grondanti di sangue, di efferatezze, di pulsioni di vendetta represse e laceranti. Nell'antefatto, Tristano aveva fatto recapitare a Isotta la testa mozza del suo promesso sposo, da lui stesso ucciso in un duello in cui era stato ferito mortalmente dalla spada avvelenata di lui; quindi, era stato costretto a rifugiarsi sotto falso nome

\footnotetext{
2 ARThUR Groos, Between memory and desire: Wagner's libretto and late Romantic subjectivity, in Richard Wagner: Tristan und Isolde, a cura di Arthur Groos, Cambridge, Cambridge University Press, 2011, p. 41.
} 
presso Isotta, l'unica persona in grado di curarlo. Isotta lo aveva riconosciuto e avrebbe voluto rendere giustizia all'amato defunto, ma al momento opportuno non aveva trovato la determinazione per trafiggerlo, in preda a quell'attrazione inconscia che la vicenda rappresentata fa emergere alla coscienza. All'inizio del dramma Isotta può così vedere in Tristano un eroe ambiguo, ad un tempo «eletto» e «perduto", "prode» e «vile» (I.2). La riuscita della missione di Tristano implicherebbe la separazione da Isotta - destinata sposa a re Marke - e invece comporta il riavvicinamento tormentoso dei due e l'avvio di quel processo d'identificazione interiore che è essenziale al compiersi della loro unanime redenzione, al loro affrancarsi dalla schiavitù del giorno e alla celebrazione della sublime verità nell'oscurità della notte.

Le relazioni tra Tristano e re Marke (lo zio, il re senza prole che ha scelto il giovane principe senza terra come amico, figlio adottivo ed erede) s'avviluppano anch'esse in un intrico di dualismi. Valutato col metro delle leggi e degli usi, il tradimento di Tristano nei confronti di Marke è colpa grave, che infrange i vincoli dell'amicizia e travalica i limiti dell'onore e del contegno che la società cavalleresca impone al vassallo: e di questo, appunto, il re desolato e affranto chiede ragione. Mentre per Tristano, passato attraverso l'esperienza del filtro e della passione e condotto dalla personificazione dell'amore, "Frau Minne», sulla via di un destino inesorabile, la sua attitudine verso il re è parte dello svelamento di un arcano inspiegabile, che le creature del giorno e il mondo mai potranno conoscere. ${ }^{3}$ Ad esse, conscio del divario di conoscenze e di esperienze che li separa, Tristano non può che rivolgersi con gli occhi pieni di "compassione» (II.3). Insomma, la progettata unione matrimoniale del sovrano e della figlia del re d'Irlanda è un fatto che riguarda la realtà storica. Viceversa, la passione fatale di Tristano e Isotta, nella sua indifferenza alla realtà, è condizione essenziale della palingenesi dell'umano.

Pure l'agente cardine della narrazione e dello sviluppo della sua componente interiore e mitica, il filtro magico, possiede tratti marcatamente ambivalenti: è la determinazione di bere il filtro di morte a indurre Tristano e Isotta a bere il filtro d'amore; quindi, è lo scambio delle pozioni ad opera di Brangania e sono le componenti drammatiche implicite nella simbologia del filtro - l'inganno, il rimescolamento delle carte, la ridefinizione delle posizioni tra apparire ed essere - a far emergere alla coscienza dei protagonisti la passione che da tempo agiva nel loro inconscio. E il duplice e illusorio influsso del filtro che spinge Tristano verso l'amore eterno e verso la spada di Melot e fa sì che per morire d'amore egli debba attendere Isotta negli spasimi e nell'angoscia. Al destino crudelmente duale della vicenda personale di Tristano si riferisce infatti Isotta nel duetto d'amore (II.2):

3 «TRISTAN | O König, das I kann ich dir nicht sagen; | und was du frägst, I das kannst du nie erfahren». (O re, questo I non posso io dirti; | e quel che tu chiedi, I tu non potrai mai sapere), II.3; cito la versione italiana dalla traduzione di Guido Manacorda, adottata anche nel Libretto e guida all'opera, a cura di Riccardo Pecci, qui alle pp. 50-150. 
Doch ach, dich täuschte

der falsche Trank,

dass dir von neuem

die Nacht versank:

dem einzig am Tode lag,

den gab er wieder dem Tag!

Pure, ahimè, t’illuse I il perfido filtro, I così che a te nuovamente I s'affondò la notte: I colui, che solo pensava alla morte, I il filtro donò nuovamente al giorno!

\section{Azione e immaginazione}

Il dramma di Tristano e Isotta si svolge dunque sull'asse della polarità fra azione visibile (sintetizzata nelle tematiche della legge cavalleresca, del costume, del giorno, della vita) e azione interiore (resa scenicamente percepibile attraverso il mare, il filtro, la passione d'amore, la notte, la morte), in una dinamica narrativa che comporta una progressiva interiorizzazione degli eventi. Tale polarità individua nel complesso il Tristan come forma 'privata' del dramma del puramente umano, dell'uomo nuovo, libero e redento, che è mèta costante del teatro wagneriano; laddove, invece, il ciclo del Ring des Nibelungen ne fornirebbe una rappresentazione collettiva e universale. Allo stesso tempo, il processo di interiorizzazione illumina di una luce particolare il concetto di 'azione' (Handlung) col quale Wagner volle definire la peculiarità di Tristan: ${ }^{4}$ si tratta infatti di un agire che oltrepassa gli eventi visibili e si serve di essi per rivelare l'essenza della condotta degli uomini.

Pochissimi elementi hanno a che fare esclusivamente con la dimensione visibile del dramma e sono gli eventi reali che riguardano la cornice fenomenica che dà l'avvio a ciascun atto. Neppure queste scene, tuttavia, sono esenti da ripercussioni nell'intimo dei personaggi. La battuta di caccia del re nella «chiara, deliziosa notte d'estate», per esempio, è un evento determinato unicamente sul piano sonoro, realizzato mediante il progressivo allontanarsi della musica dei corni posti dietro il fondo della scena: una musica di scena di natura diegetica, di effetto realistico tanto immediato, quanto convenzionale. Isotta, però, non ne intende il segnale concreto e riesce a percepirla soltanto come suono di natura che in modo soggettivo riconduce alle metafore dell'acqua e del viaggio per mare e, nell'impeto del desiderio, interpreta come annuncio della venuta di Tristano (II.1):

Nicht Hörnerschall

tönt so hold;

des Quelles sanft

rieselnde Welle

rauscht so wonnig daher:

${ }^{4}$ Cfr. Dahlhaus, I drammi musicali di Richard Wagner cit., pp. 65-67 e ID., La concezione wagneriana del dramma musicale [Wagners Konzeption des musikalischen Dramas, 1971], Fiesole, Discanto, 1983, pp. 129-135. 


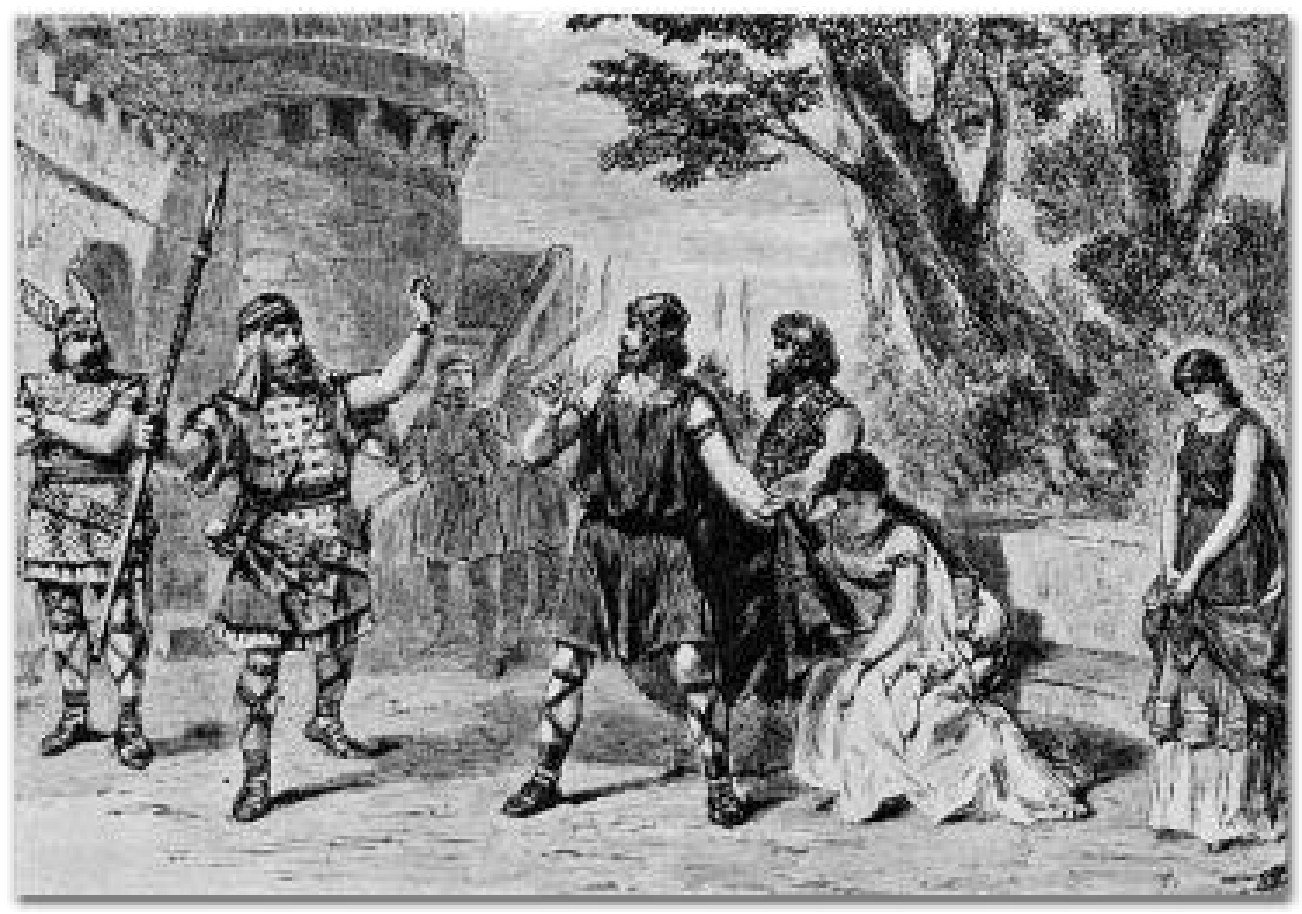

Tristan und Isolde (atto II). Incisione da un disegno per la rappresentazione a Lipsia, Stadttheater, 1882, apparsa sulle pagine del periodico «Il teatro illustrato».

wie hört' ich sie,

tosten noch Hörner?

Im Schweigen der Nacht

nur lacht mir der Quell:

der meiner harrt

in schweigender Nacht,

als ob Hörner noch nah dir schallten,

willst du ihn fern mir halten?

Non suono di corni I così gratamente suona: | della fonte la dolcemente | mormorante onda | mormora verso di noi così soavemente. I Come l'udrei, I se rimbombassero i corni ancora? | Nel silenzio della notte, I solo mi ride la fonte; | colui che m'attende I nella silente notte, | come se i corni ancora vicino a te suonassero, I vuoi tu tenermelo lontano?

Una sostituzione tanto lampante dell'immaginazione soggettiva al principio di realtà non sfugge alla musica che, proprio nel momento in cui Brangania ne svela l'illusione, ${ }^{5}$

\footnotetext{
5 «BRANGÄNE | Dich täuscht des Wunsches | Ungestüm, | zu vernehmen, was du wähnst. | (Sie lauscht.) Ich höre der Hörner Schall.» (Te illude del desiderio I l'impeto I di udire, quel che tu pensi. I (Presta ascolto.) Io odo il suono dei corni), II.1.
} 
trapassa in modo quasi impercettibile dagli ultimi echi della musica della caccia regale al suono 'di natura' interiormente sentito da Isotta e descritto nel disegno ondeggiante di clarinetto basso, violini e viole. ${ }^{6}$

ESEMPIO 1 - II.1, B ${ }^{30}$.
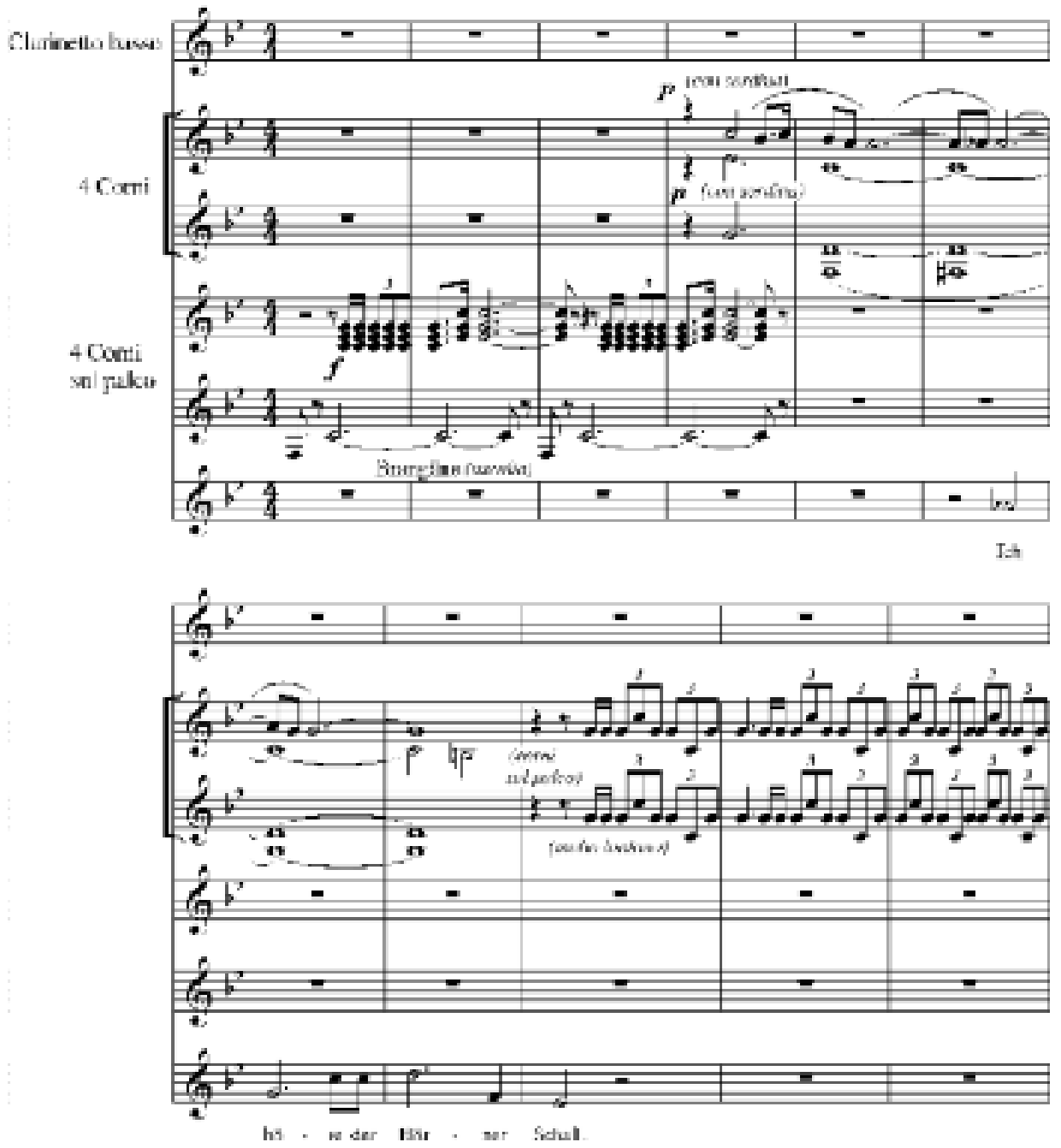

${ }^{6}$ Per un'interpretazione complessiva delle funzioni della musica 'interna' ai personaggi cfr. THOMAS GREY, In the realm of the senses: sight, sound and the music of desire in "Tristan und Isolde», in Richard Wagner: Tristan und Isolde cit., pp. 65-94.

7 Si cita dalla prima edizione della partitura: RICHARD WAGNER, Tristan und Isolde, Leipzig, Breitkopf \& Härtel, s.a. [1860], n.e. 10000; il luogo s'individua mediante l'atto e la scena, seguiti dal riferimento alla lettera di chiamata in apice, a destra (se la segue), a sinistra (se la precede). 

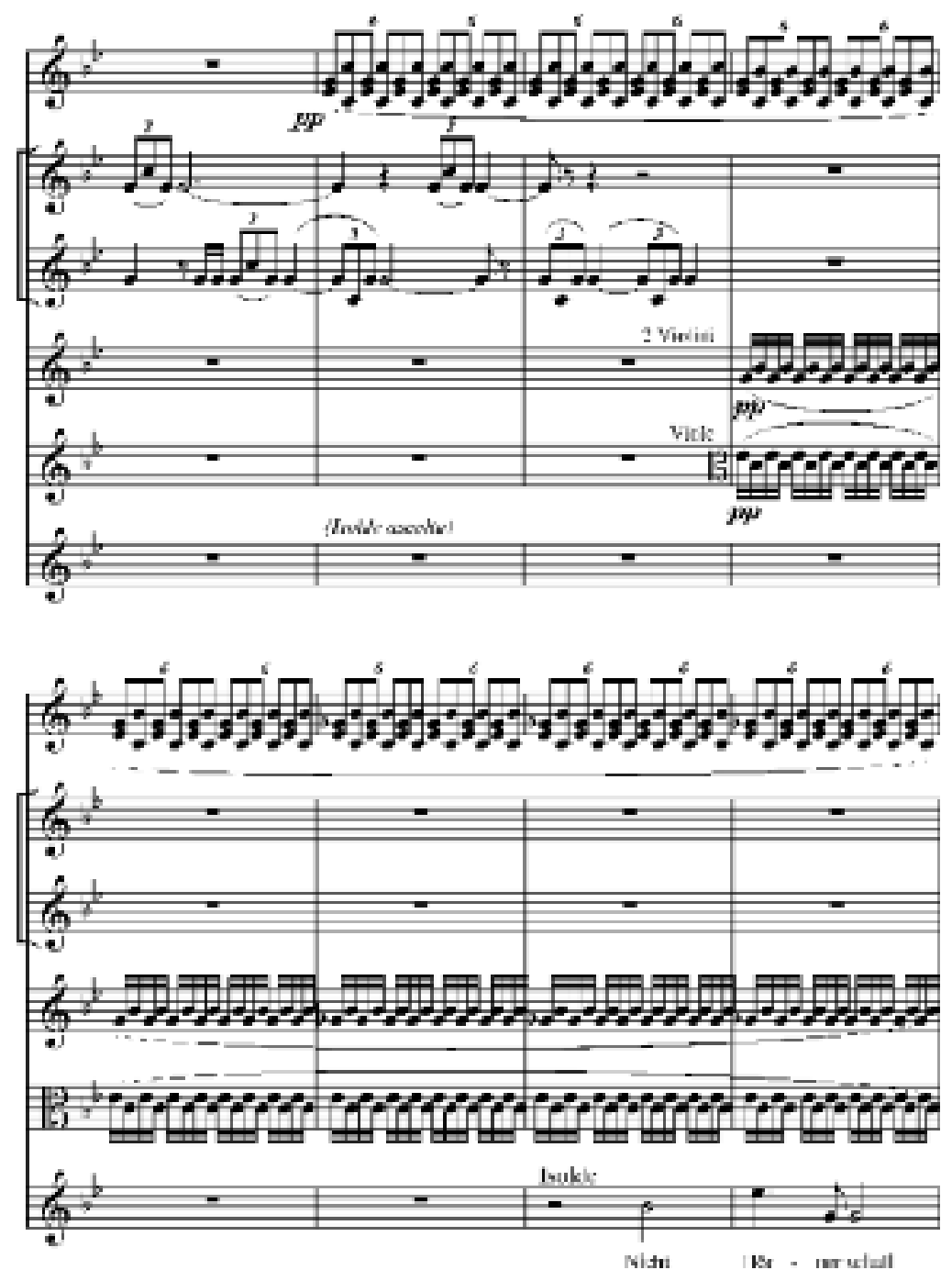

\section{L'arte della transizione musicale}

L'opera di determinazione del dramma e la sostanza poetica della musica di Tristan und Isolde risiedono soprattutto nelle relazioni sottili che fanno dell'azione visibile una funzione accessoria alla rappresentazione dell'azione interiore. Wagner stesso evidenzia questo concetto nella lettera a Frédéric Villot sulla Musica dell'avvenire (1861):

Uno sguardo all'estensione di questo poema Le mostra subito che la medesima determinatezza dettagliata, con la quale il poeta, trattando un soggetto storico, si mette all'opera per ispiegare le connessioni esterne dell'azione, con pregiudizio della manifestazione chiara dei motivi interni, io ardiva applicarla esclusivamente a questi motivi. [...] Tutta l'azione commovente si 
rivela unicamente per ciò, che essa è richiesta e provocata dal più profondo dell'anima, ed appare manifesta così come l'intimo l'ha profondamente immaginata. ${ }^{8}$

L'espressione musicale dell'intenzione poetica del dramma in Tristan non può prescindere dunque dalla dimensione profonda dell'azione; tanto che, istituendo col testo poetico un rapporto a tratti intermittente, la musica rinuncia a rendere esplicite alcune delle sue articolazioni di senso più superficiali e determinate. Carl Dahlhaus, riferendosi al contenuto della lettera di Wagner a Mathilde Wesendonck del 29 ottobre 1854, in cui il musicista svela il «segreto» della propria concezione della forma musicale, ha individuato la peculiarità compositiva di Tristan nell' «arte della transizione», ossia nella tendenza a costituire fisionomie motiviche fluttuanti, per cui «non soltanto i motivi sono riferiti l'uno all'altro [...], bensì straripano l'uno dentro l'altro e sconfinano poi nell'indefinito e nell'intangibile». ${ }^{9}$ Wagner, da parte sua, descriveva tale tecnica come modalità di trapasso progressivo, capace di regolare anche ciò che è "brusco e repentino", purché «l'animo sia stato tanto accuratamente predisposto alla transizione improvvisa da esigerla esso stesso». ${ }^{10}$

In Tristan la cosiddetta arte della transizione agisce da principio di costruzione a vasto raggio nei momenti di maggiore intensità musicale della partitura, come il preludio all'atto primo, il grande duetto d'amore nell'atto secondo (per Wagner il «capolavoro supremo nell'arte della transizione sottile e graduale»), il Liebestod di Isotta nell'atto terzo: brani nei quali è possibile ravvisare i processi di mediazione musicale tra piani narrativi esterni e piani narrativi interni ai personaggi.

Prendiamo, per esempio, la parte centrale del duetto Tristano-Isotta dell'atto secondo, quella che attacca con l'invocazione alla notte «O sink hernieder, I Nacht der Liebe» (Oh scendi quaggiù, I notte dell'amore). Nell'economia del duetto rappresenta la sezione per così dire cantabile, distinta sia dalla parte dialogica che la precede, sia da una sorta di quasi-cabaletta, dal ritmo via via più animato, che la segue a partire da «O ew'ge Nacht, I süsse Nacht!» (O notte eterna, I dolce notte!). In «O sink hernieder» è svolto il tema fondamentale del dramma, quello della liberazione dell'io dal mondo sancita dall'unione fisica dei corpi degli amanti, protratta nel delirio dei sensi e, infine, invocata nella suprema comunione della morte. Il testo poetico e la musica si presentano sotto forma di dialogo prolungato tra Tristano e Isotta, il cui flusso è interrotto dalle interpolazioni costituite dagli ammonimenti della voce di Brangania e dall'anticipazione musicale del motivo del Liebestod, della Morte d'amore, che qui si determina nel suo significato 'poetico' alle parole di Tristano «So starben wir, I um ungetrennt, I ewig einig | ohne End'» (Così siamo morti: | per inseparati, | eternamente congiunti, | senza fine).

8 RichaRd Wagner, Musica dell'avvenire. Ad un amico francese (Fr. Villot) [Lettre sur la Musique. À Monsieur Frédéric Villot, 1861; Zukunftsmusik. Brief an einen französischen Freund, 1861], trad. di Luigi Torchi, Torino, Bocca, 1893, 1924², p. 68.

9 DahlHaus, I drammi musicali di Richard Wagner cit., p. 79.

${ }^{10}$ Lettera a Mathilde Wesendonck del 29 ottobre 1859, in Richard Wagner an Mathilde Wesendonk, cit., pp. 188-194, cit. in DahlHaUs, I drammi musicali di Richard Wagner cit., p. 73. 
ESEMPIO 2 - II.2, $\mathrm{Aa}^{31}$, duetto Tristano-Isotta.

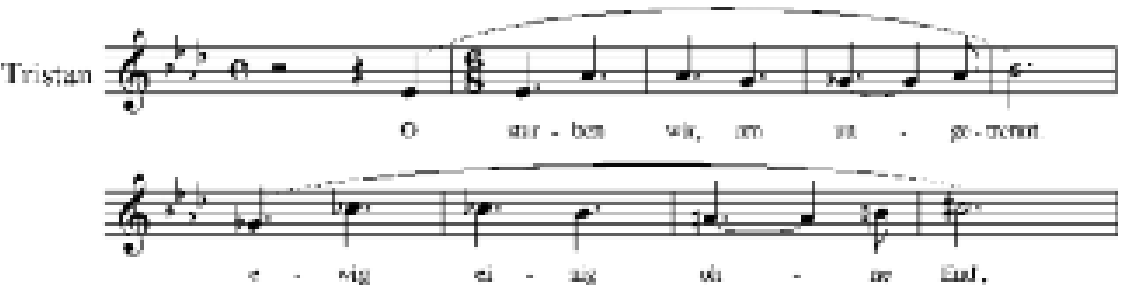

I discorsi rapiti degli amanti, sul piano delle relazioni tonali, si tengono assai discosti dalle intromissioni della voce di Brangania, tanto quanto, sul piano spaziale, la scena del duetto è separata dal luogo da cui proviene la voce di Brangania. Gli ammonimenti di quest'ultima circa l'incombere del giorno e l'approssimarsi dell'ora del risveglio, opponendosi alle apologie del sonno eterno e della morte pronunciate da Tristano e Isotta, agiscono da potenti richiami alla realtà della vita. Proprio gli avvertimenti di Brangania consentono infatti di gettare un ponte narrativo fra il duetto e la scena successiva, fino al punto in cui la realtà dell'azione esteriore si presenta col volto inorridito con cui re Marke e Melot sorprendono i due amanti immersi nel loro mondo di sogno, suscitando per reazione l'invettiva convulsa di Tristano. ${ }^{11}$ Dal punto di vista del contenuto drammatico, perciò, il duetto è un brano incompleto, interrotto nel suo momento risolutivo da un principio di realtà che produce un anticlimax rispetto alla sua tensione protratta (enormemente più dilatata che in qualsiasi altra scena) all'evasione dal reale.

Gli elementi musicali preminenti di «O sink hernieder» sono quelli in cui tradizionalmente l'analisi musicale riconosce le connotazioni motiviche del Sogno d'amore, presente nelle prime battute del canto (es. 3), e dell'Estasi dei sensi (es. 4), proposto per la prima volta in coincidenza con la descrizione di Isotta della sua congiunzione con Tristano, «Herz an Herz dir, I Mund an Mund» (il mio cuore sul tuo, I sulla tua la mia bocca):

ESEMPIO 3 - II.2, ${ }^{15} \mathrm{~V}$, motivo del Sogno d'amore.

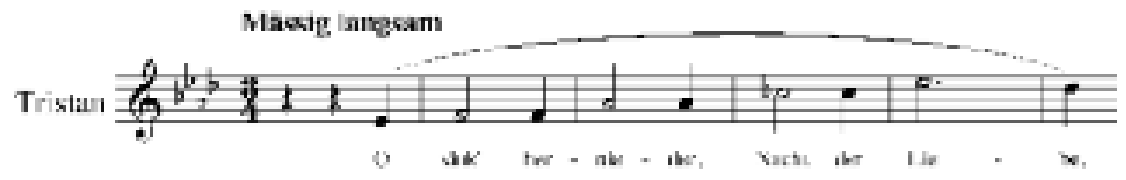

ESEMPIO 4 - II.2, V ${ }^{36}$, motivo dell'Estasi dei sensi.

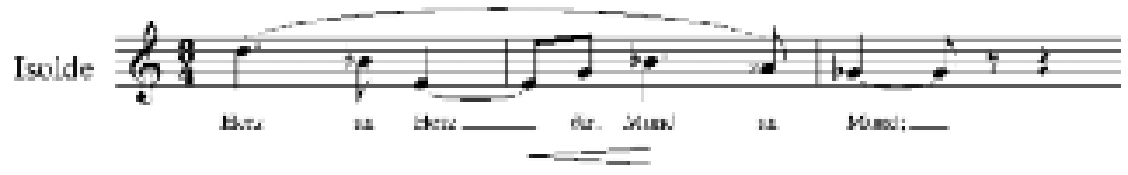

11 «TRISTAN | Tagsgespenster! | Morgenträume! I täuschend und wüst! | Entschwebt! Entweicht! (Spettri del giorno! | Sogni del mattino | illusori ed orridi! | Sparite! Andatevene!), II.3. 


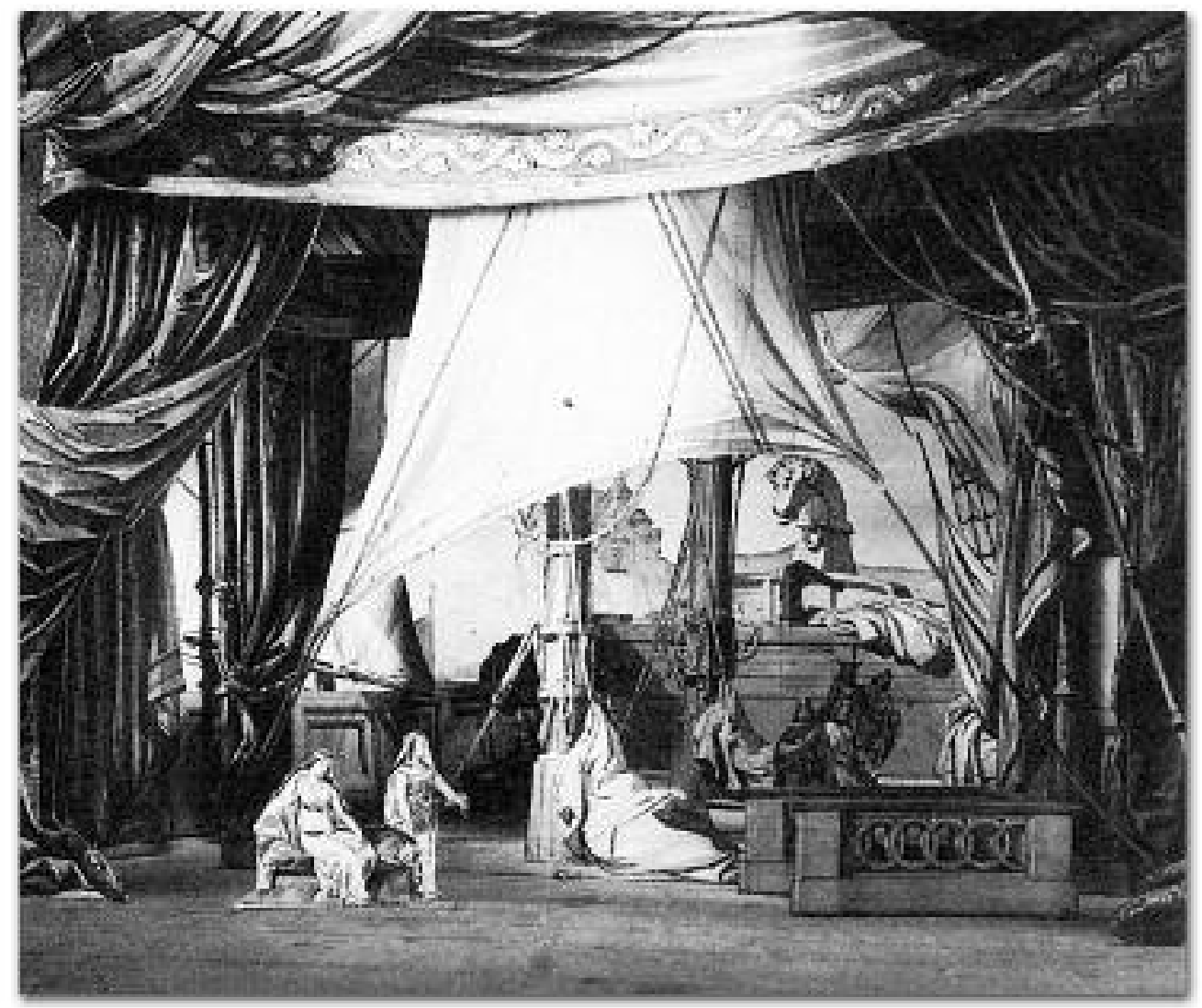

Modellino del bozzetto scenico di Angelo Quaglio II (1829-1890) per la prima assoluta di Tristan und Isolde al Königliches Hof- und Nationaltheater di Monaco, 1865.

Il Sogno d'amore e l'Estasi dei sensi appartengono alla sfera dei 'motivi espressivi' di Tristan, quelli che s'incardinano sul lato interiore dell'azione drammatica e sfuggono a determinazioni musicali e a denominazioni stabili. Nella sezione centrale del duetto essi convivono con altri motivi appartenenti alla sfera delle connotazioni 'allegoriche' - come i motivi del Giorno e della Morte - tendenti, all'opposto, a tradurre in figurazioni musicali dirette e univoche le metafore narrative del testo poetico. ${ }^{12}$

Il motivo dell'Estasi, variamente denominato anche come «motivo della pace d'amore» oppure «motivo del sopore», presenta caratteri e funzionalità drammatiche singolari. Innanzi tutto, è nato prima del testo poetico, tanto che lo si potrebbe definire l'idea

12 Per la classificazione dei motivi del Tristan in 'espressivi' e 'allegorici', che confuta alcuni giudizi di Theodor W. Adorno (cfr. Versuch über Wagner [1956], trad. it. Wagner, Torino, Einaudi, 2008, pp. 39-58) sulla tecnica motivica nei drammi wagneriani, cfr. DAHLHAUS, I drammi musicali di Richard Wagner cit., p. 78. 
primigenia di Tristan: Wagner lo annota per la prima volta in un abbozzo del dicembre 1856, coevo alla lettera nella quale comunica a Marie Wittgenstein che una nuova e irresistibile attrazione per il soggetto del romanzo di Tristano e Isotta lo distoglie dagli sforzi fin lì compiuti per districarsi con la realizzazione della partitura del Siegfried. In secondo luogo, la sua connotazione formale è più quella di un tema che di un Leitmotiv. Nella configurazione estesa, che si sente con funzione d'interludio sinfonico tra la prima interpolazione di Brangania e la ripresa sommessa del dialogo dei due amanti (es. 5), si presenta infatti come melodia di otto battute, poi ripresa e sviluppata nel contesto del duetto secondo criteri di tipo sinfonico.

ESEMPIO 5 - II.2, ${ }^{27} \mathrm{Z}$.
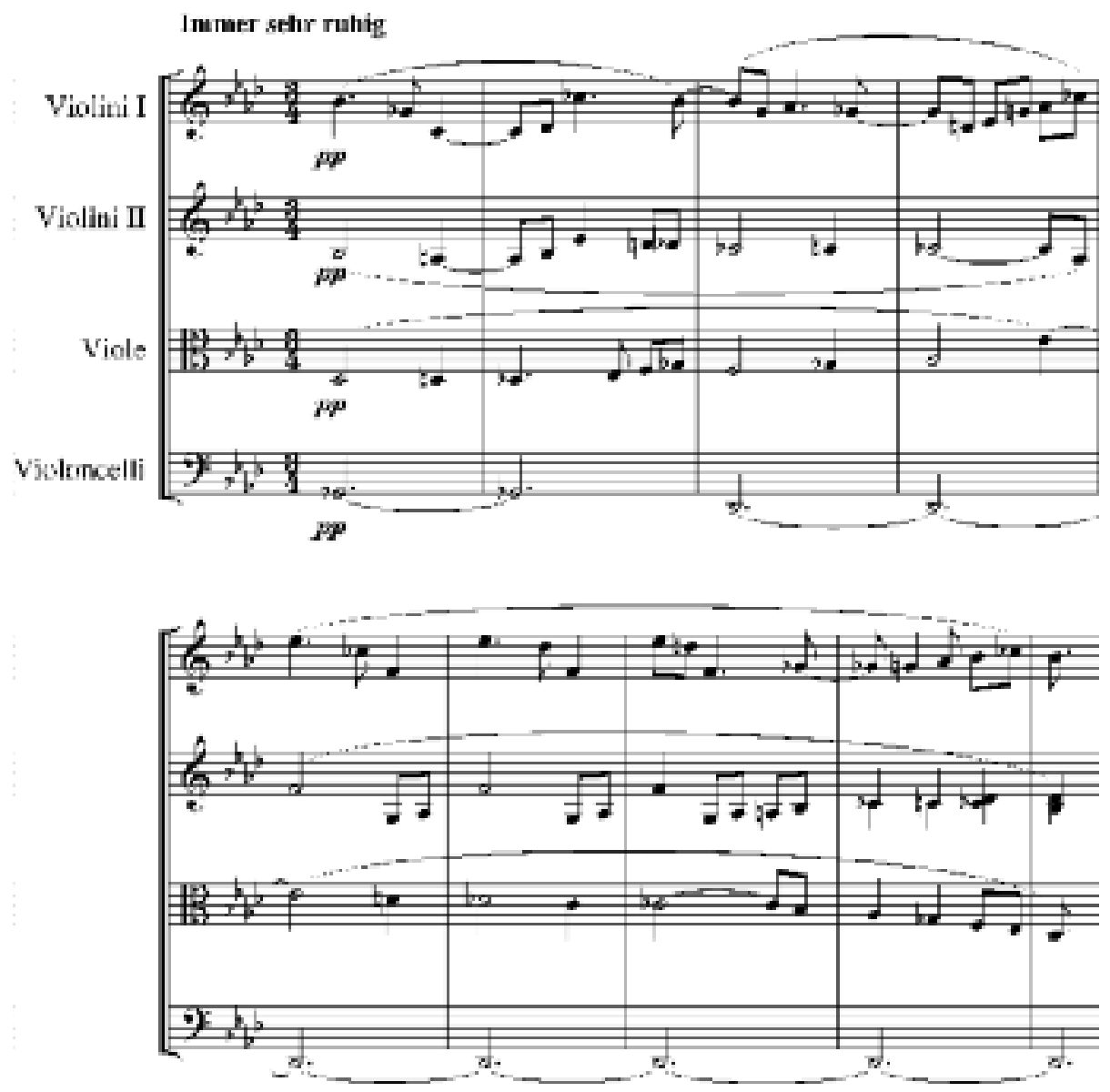

Dopo l'interpolazione, il motivo dell'Estasi definisce l'episodio in cui i due amanti, incuranti degli ammonimenti di Brangania, si perdono a vagheggiare il compimento supremo dell'amore nella morte. Nel dialogo che attacca con le parole di Isotta «Lausch, Geliebter!» (Odi, mio caro!), la melodia dell'Estasi viene però emancipata dalla natura 
'poeticizzante' delle otto battute dell'interludio sinfonico e calata in una nuova entità 'prosodica', di tipo bi-motivico, costituita per la prima metà dalle battute 1-4 dell'Estasi e nella seconda metà, alla risposta di Tristano «Laß mich sterben!» (Lasciami morire!), dal cosiddetto secondo motivo della Morte, ossia dal motivo associato all'idea della morte liberatrice dalle sofferenze del giorno e della vita (es. 6).

ESEMPIO 6 - II.2, ${ }^{19}$ Z, combinazione del motivo dell'Estasi e del motivo della Morte.

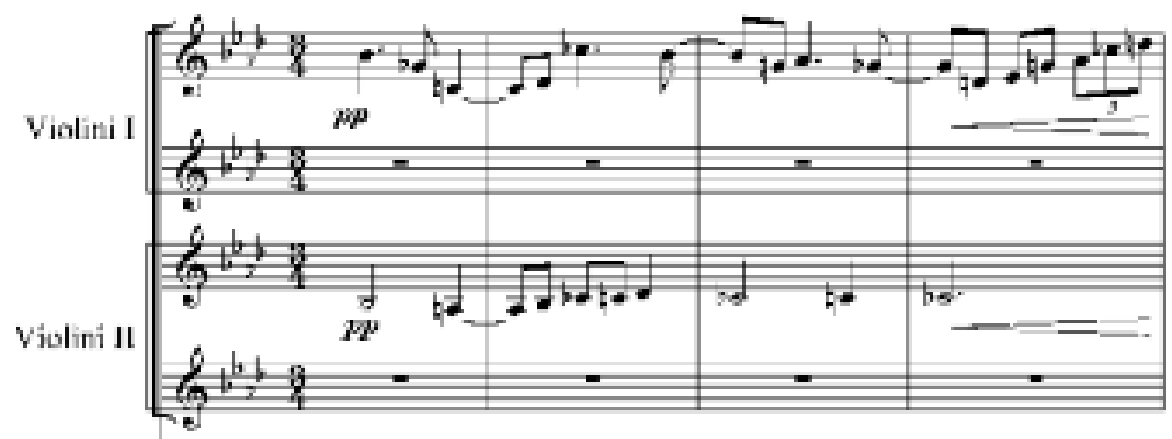

Isolde (dolesmente)
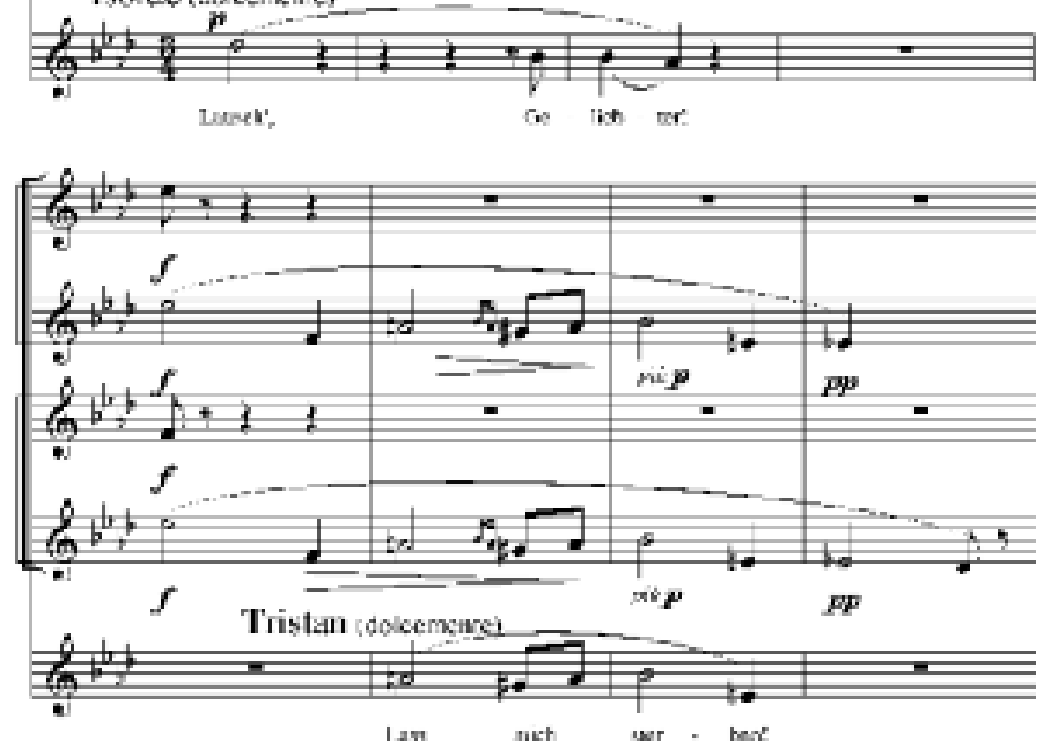

Questa combinazione si mantiene invariata in tutto il corso del dialogo, così che la 'melodia finita' dell',Estasi - espressione del lato interiore dell'azione - vi si attesta in modo inequivocabile come 'melodia infinita', prodotta dalla congiunzione con un motivo allegorico, realmente determinato come Leitmotiv dal testo poetico, e mediante quest'ultimo è predisposta alla dialogizzazione del tessuto musicale. In seguito, ai passi «Unsre Liebe?» (Il nostro amore?) e «Stürb ich nun ihr» (Morissi anche io d'amore) di Tristano, «Doch unsre Liebe» (Ma il nostro amore) di Isotta e «Was stürbe dem Tod» (Che cosa soccomberebbe alla morte) di Tristano l'entità bi-motivica di «Lausch, Geliebter!» 


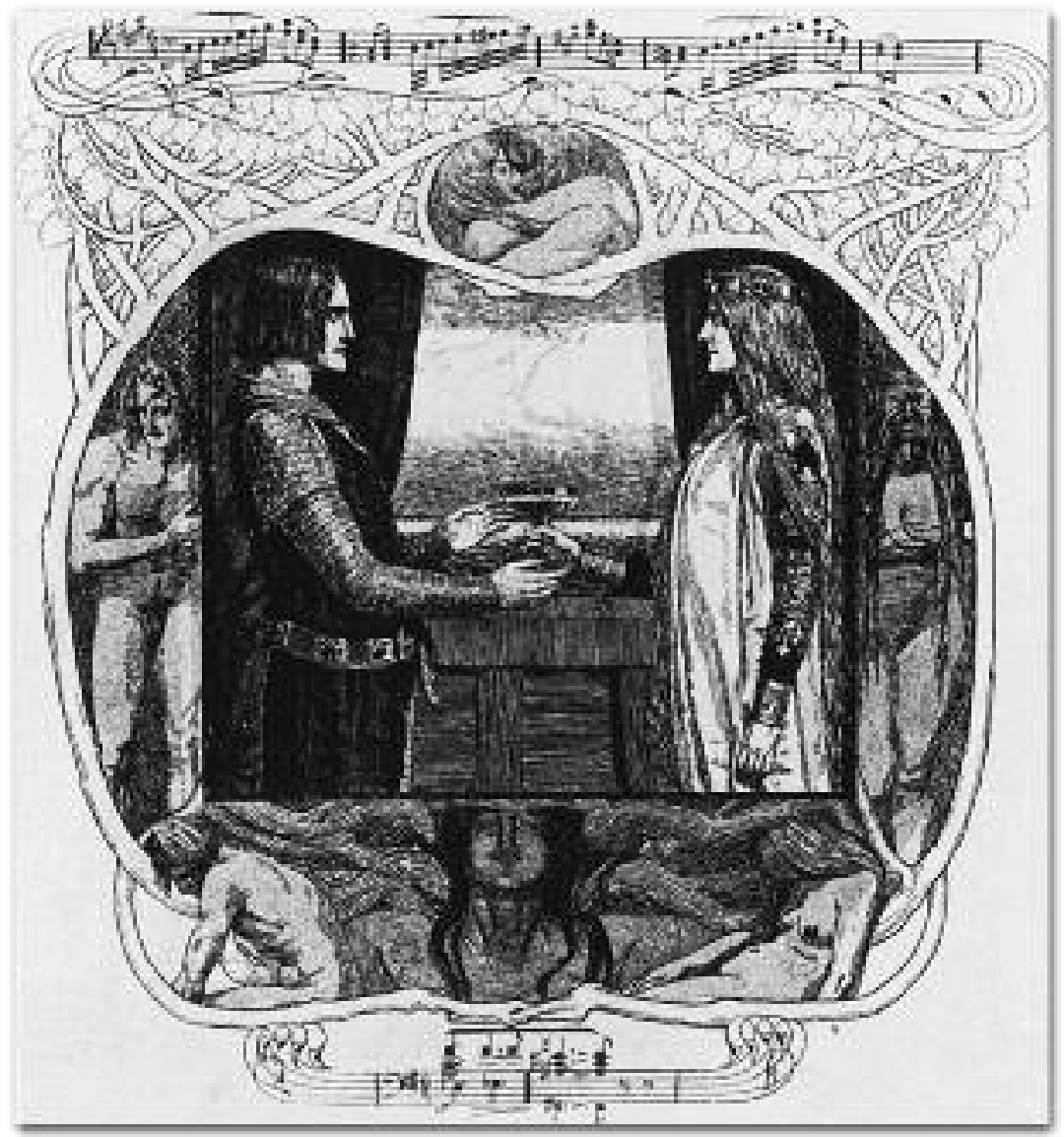

Tristano e Isotta (atto I). Incisione dallo spartito Ricordi, Milano 1907.

è sottoposta a elaborazioni musicali che, pur conservando invariate l'associazione e la sequenza degli elementi, investono la componente del motivo dell'Estasi, sottoposta a una serie progressiva di varianti. All'interrogativo «wär Tristan der Tod gegeben?» ("potrebbe essere data a Tristano la morte?») l'ultima variante del motivo dell'Estasi entra in una nuova doppia entità insieme al motivo del Giorno; dopo di che sfocia nell'anticipazione del Liebestod citata all'esempio 2. Questo motivo funge quindi da collegamento 'logico' tra la prima sezione del cantabile e la quasi-cabaletta di «O ew'ge Nacht I süsse Nacht!» (O notte eterna, I dolce notte!). Si determina così un percorso che nella sequenza delle varianti segue la logica strettamente musicale dello sviluppo dell'idea fondamentale, svincolata però dalla connotazione diretta del testo poetico.

È una dimostrazione di come in Tristan - molto più che nei drammi del ciclo nibelungico - l'avvicendamento dei motivi nel tessuto sinfonico si connetta secondo linee frastagliate che rispecchiano le intermittenze logiche dei piani narrativi e le sottigliezze 
di dialoghi che gli interlocutori svolgono sotto forma di flussi dell'intimo. Ciò comporta anche il costituirsi di una rete parallela di relazioni musicali. Un caso di nesso leitmotivico di tipo transitivo nell'àmbito dei motivi connessi con la sfera interiore si individua nel nesso sotterraneo che lega il motivo della Desolazione dell'es. 7 B (il frammento di scala diatonica Sol-Lab-Sib-Do), che introduce la prima scena dell'atto terzo e che è parte significativa del suo materiale sonoro, al motivo del Desiderio (il frammento di scala cromatica Sol\#-La-La\#-Si), che si sente in testa al preludio e che è parte cospicua della materia sonora dei primi due atti (es. $7 \mathrm{~A}$ ).

ESEMPIO 7 A - Einleitung, bb. 1-3.

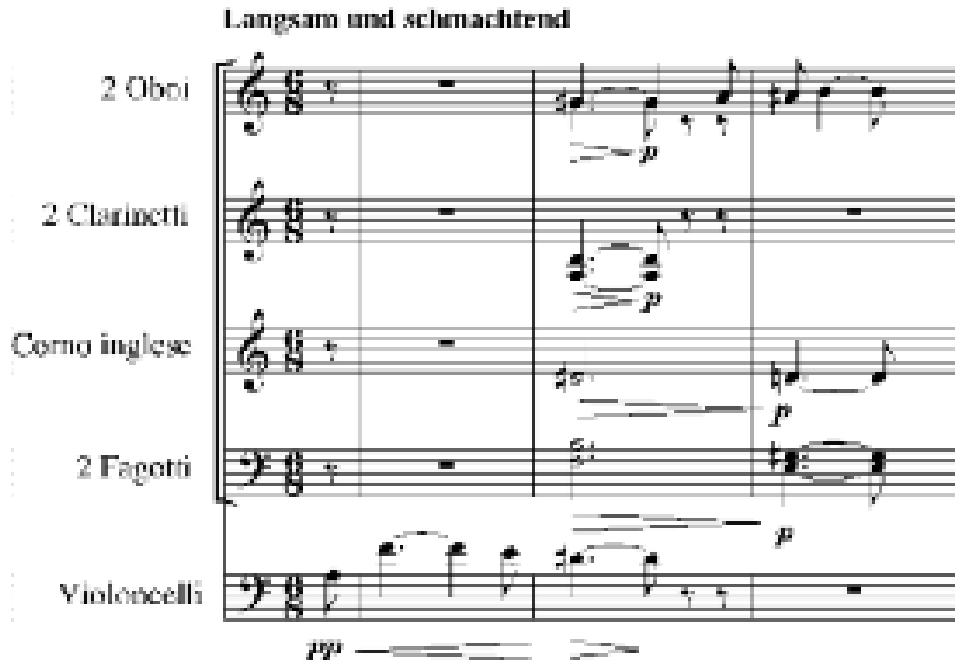

ESEMPIO 7 B - III.1, bb. 1-4.

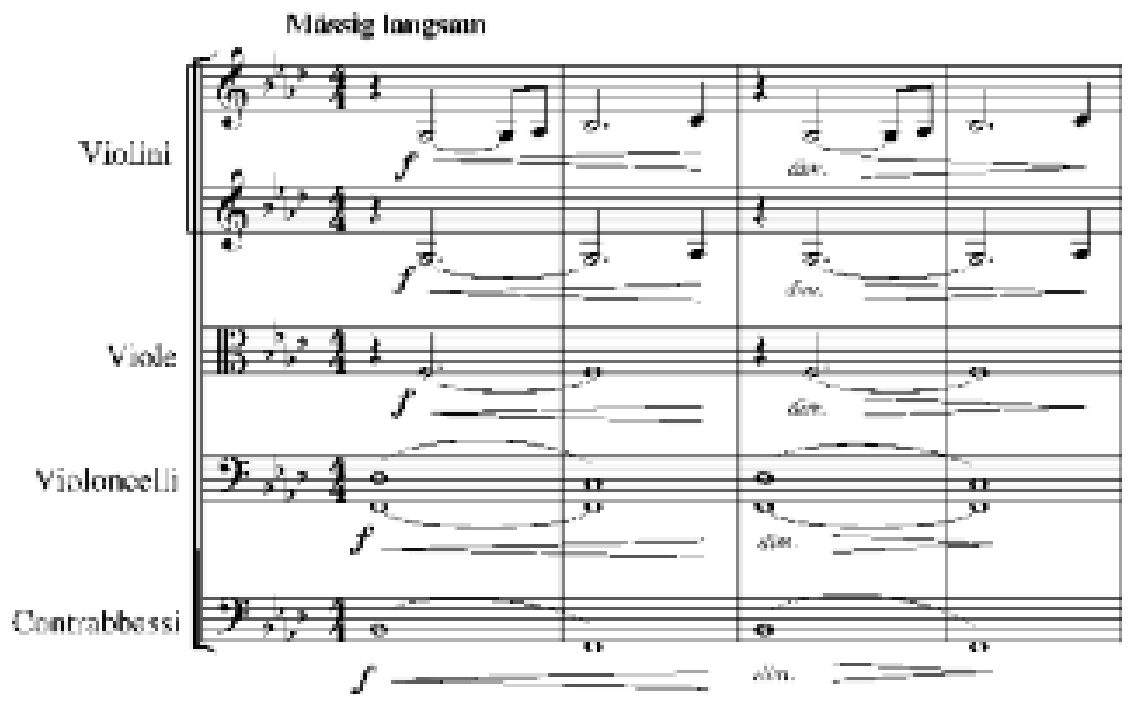


I due Leitmotive, apparentati dalle somiglianze di carattere musicale (la configurazione aperta dei frammenti scalari, la sequenza suono lungo-suono breve dell'attacco, l'indugio sulla penultima nota sotto forma di appoggiatura inferiore), eppure diversi, creano un'associazione narrativa che riguarda l'azione invisibile esercitata dal filtro magico su Tristano: il desiderio indotto gli ha procurato la sofferenza di un amore incompiuto e la bramosia inappagata di renderlo eterno con la morte. Un significato rinforzato dalla struggente malinconia della musica 'dal vero' della canzone del pastore, suonata da dietro il palcoscenico dal corno inglese e nella parte iniziale della scena alternata agli episodi basati sul motivo della Desolazione. Fra l'altro, è stato osservato come Wagner contamini il topos ingenuamente pastorale - da ranz des vaches - della melodia del corno inglese con il cromatismo estenuante dell'idioma sentimentale di Tristan, facendone così una componente musicale psicologica del delirio di Tristano, una sorta di voce oggettivata della sua vana attesa del ritorno di Isotta. ${ }^{13}$

Sia il motivo dell'Estasi nel duetto, sia la relazione non determinata dal testo poetico tra motivo del Desiderio e motivo della Desolazione, nonché la relazione stilisticamente 'forzata' di quest'ultimo con la musica del pastore, sono «azioni musicali diventate visibili», secondo la definizione che nel 1872 Wagner avrebbe voluto introdurre per tutelarsi dai fraintendimenti suscitati dal concetto di Musikdrama. I metodi pendolari di impiego della musica in Tristan la illustrano tuttavia con uno stacco sensibile rispetto alla teoria e alla prassi del Musikdrama inteso come azione realizzata attraverso la musica, come risultato della mediazione tra l'intenzione poetica e l'espressione musicale. La duplice funzione dell'azione musicale di Tristan - ora determinata nel dramma, ora organizzata in articolazioni formali in sé finite, di senso ambivalente rispetto al dramma - conduce infatti a una situazione drammaturgica particolare, che da una parte istituisce nessi con l'estetica tardo romantica della musica assoluta e, d'altra parte, risente fortemente della lettura appassionata del Mondo come volontà e rappresentazione e dell'influsso che il musicista trasse dalla metafisica della musica di Schopenhauer per insinuare nel dramma il dualismo tra l'invisibile «volontà» agita dalla musica e il suo rispecchiamento nella «rappresentazione» visibile. Una bipolarità che costituisce la base estetica della duplicazione dei piani narrativi che siamo venuti illustrando.

\section{Ewig, ewig ein}

Nel duetto dell'atto secondo, un filo sottile lega i passi in cui i due protagonisti si perdono in elucubrazioni sul valore eterno dell'uno, dell' «ein", dell'indissolubile unità psichica e fisica generata dal vincolo d'amore. Il tema è fatto affiorare da Isotta nell'ondata degli «impetuosi amplessi» coi quali all'inizio Tristano e Isotta guadagnano il

13 Cfr. GREY, In the realm of the senses cit., pp. 77-79 e LaWRENCE KRAMER, Opera and Modern Culture: Wagner and Strauss, Berkeley-Los Angeles-London, University of California Press, 2004, pp. 224-225. 
proscenio, ${ }^{14}$ ed è quindi sviluppato nelle riflessioni sulla congiunzione «und» che precedono l'anticipazione del Liebestod. ${ }^{15}$

Nella parte antecedente a quella che qui si è definita sezione 'cantabile', il passo «Himmelhöchstes | Weltentrücken!» (Altissimo celeste I rapimento dal mondo!) introduce nel testo poetico una struttura dialogica peculiare, basata sullo scambio incrociato di parole e concetti, che Isotta e Tristano letteralmente si rinviano dall'uno all'altro. Un sequenza dialogica analoga si trova nella parte centrale del duetto, nei due passi che seguono le interpolazioni di Brangania e che contengono le risposte elusive degli amanti ai suoi appelli. L'idea poetica dell'intreccio delle identità, del desiderio di diventare una cosa sola, in questo caso è realizzata sia sul piano testuale che su quello musicale. Le sticomitie verbali del primo episodio ritornano infatti in perfetta identità di senso e spesso di dettato, ma a parti rovesciate, nel secondo episodio:

I.

$\begin{array}{ll}\text { ISOLDE } & \text { Lausch, Geliebter! } \\ \text { TRISTAN } & \text { Lass mich sterben! } \\ \text { ISOLDE } & \text { Neid'sche Wache! } \\ \text { TRISTAN } & \text { Nie erwachen! } \\ \text { ISOLDE } & \begin{array}{l}\text { Doch der Tag } \\ \text { muss Tristan wachen? }\end{array} \\ \text { TRISTAN } & \begin{array}{l}\text { Lass den Tag } \\ \text { dem Toden weichen! }\end{array}\end{array}$

I.

ISOTTA Odi, mio caro!

TRISTANO Lasciami morire!

ISOTTA Invidiosa vigilia!

TRISTANO Mai più svegliarsi!

ISOTTA Eppure il giorno dovrà svegliare Tristano?

TRISTANO Lascia che il giorno ceda alla morte!
II.

TRISTAN Soll ich lauschen?

ISOLDE Lass mich sterben!

TRISTAN Muss ich wachen?

ISOLDE Nie erwachen!

TRISTAN Soll der Tag

noch Tristan wecken?

ISOLDE Lass den Tag

dem Toden weichen!

II.

TRISTANO Devo prestare ascolto?

ISOTTA Lasciami morire!

TRISTANO Debbo vegliare?

ISOTTA Mai più svegliarsi!

TRISTANO Dovrà il giorno

ancora svegliare Tristano?

ISOTTA Lascia che il giorno

ceda alla morte!

In entrambi i casi la musica si basa sull'elemento bi-motivico costituito dalla sintesi dei motivi dell'Estasi e della Morte. Dal punto di vista dell'articolazione formale, il secondo episodio si configura dunque come ripresa variata del primo. Tuttavia, l'ulteriore processo di sviluppo del motivo dell'Estasi, così come la progressiva accelerazione agogica mediante la quale il secondo episodio conduce l'intero cantabile a "O ew'ge Nacht» ne fanno una nuova elaborazione e un'intensificazione del nucleo motivico-drammatico

\footnotetext{
14 «ISOLDE | Himmelhöchstes | Weltentrücken! | Mein! Tristan mein! I Mein und dein! | Ewig, ewig ein!» (Altissimo celeste I rapimento dal mondo! I Mio! Tristano mio! I Mio e tuo! I Eternamente, eternamente uno!)

15 «ISOLDE | Doch unsre Liebe, | heisst sie nicht Tristan | und - Isolde? I Dies süsse Wörtlein: und, I was es bindet, I der Liebe Bund, I wenn Tristan stürb, I zerstört' es nicht der Tod? (Ma il nostro amore I non si chiama Tristano | e... Isolda? | Questa dolce paroletta: $e$ | quel ch'essa congiunge, | questo vincolo d'amore, | se Tristano morisse, I non verrebbe distrutto dalla morte?).
} 
fondamentale del duetto. In questo caso, l'azione musicale divenuta visibile e l'artificio della transizione da essa attuata sono motivati dal testo poetico, a sua volta concepito in vista della realizzazione delle potenzialità drammatiche della musica.

In Tristan, dunque, la sintesi, l'eternamente uno, è raggiunta soltanto attraverso l'intreccio inestricabile delle unità di senso, mediante il contrappunto dinamico delle entità motiviche e dei processi psichici. La verità del dramma è quindi una verità indicibile e Tristano, che ad essa si consacra, ne è perfettamente conscio: "Des Schweigens Herrin I heißt mich schweigen» (La signora del silenzio I m'ordina il silenzio, I.5). Su queste basi Wagner interpreta il soggetto del romanzo medievale di Gottfried von Straßburg in una dimensione mitica. Lo sottrae alla dinamica dei conflitti umani e ai confini ristretti di ciò che è storicamente e eticamente determinato e ne fa la rappresentazione della sfera del profondo, il cui scopo è l'eternità, la dimensione a-storica, l'incondizionato. Tristan und Isolde, infatti, non sviluppa il dramma dell'onore mortificato da un amore fuorilegge, bensì il tema - fondamentale per Wagner - della frattura insanabile tra le apparenze della vita diurna e l'assoluta verità mistica della notte, simbolo della totalità nella quale la forza suprema dell'amore riunisce i singoli individui e li redime dalla finitezza delle loro nature.

Ancor più della morte di Tristano - determinata da un conflitto umano e nell'apparenza scenica favorita dalla ferita tangibile infertagli da Melot - è la dolce trasformazione di Isotta sul cadavere di lui a dar forma scenica alla metafora dello smarrimento dell'individuo e della sua ricongiunzione nel tutto del cosmo, del naufragio «nell'armonia sonora» ${ }^{16}$ e del conseguimento definitivo dell'assoluto, della felicità, dell'eternità, dell' «ewig ein». La sublime bellezza del Liebestod - che Wagner definisce anche Verklärung, trasfigurazione - e dellla melodia "così meravigliosa e sommessa, [...] tutto esprimente», che "verso l'alto si libra», ${ }^{17}$ svincolata dall'intreccio con le allegorie motiviche della vicenda esteriore, diventa il compimento sonoro della voluttà, del superamento dei confini del mondo e del conseguimento dell'infinito, di cui è artefice pienamente cosciente il personaggio dominante di Isotta, colei che vive al di là della morale. Con ciò, la musica compie un'ultima azione: l'assoluto del destino d'amore d'Isotta si fonda sul potere incommensurabile e sull'effetto ipnotico della musica assoluta, la sola in grado di superare il conflitto io/mondo e la dualità tra il condizionato delle basi storico-sociali che fanno da cornice all'azione visibile di Tristan e la loro incondizionata interiorizzazione sotto specie di poesia.

Concludo con un rilievo più generale di poetica. Un appunto segnato nel Libro bruno nel maggio 1868 a proposito di un progetto intitolato Die Sieger (I vincitori) ci fa attribuire un valore quasi programmatico alla trasfigurazione musicale di Isotta. Dal-

16 «ISOLDE | In dem wogenden Schwall, | in dem tönenden Schall, | in des Weltatems | wehendem All, - | ertrinken, I versinken, - | unbewusst, - | höchste Lust! (Nell'ondeggiante oceano I nell'armonia sonora, I del respiro del mondo I nell'alitante Tutto... I naufragare, | affondare... I inconsapevolmente... I suprema letizia!), III.3.

17 «ISOLDE | diese Weise, | die so wunder- | voll und leise | [...] | alles sagend, | [...] | auf sich schwinget», III.3. 


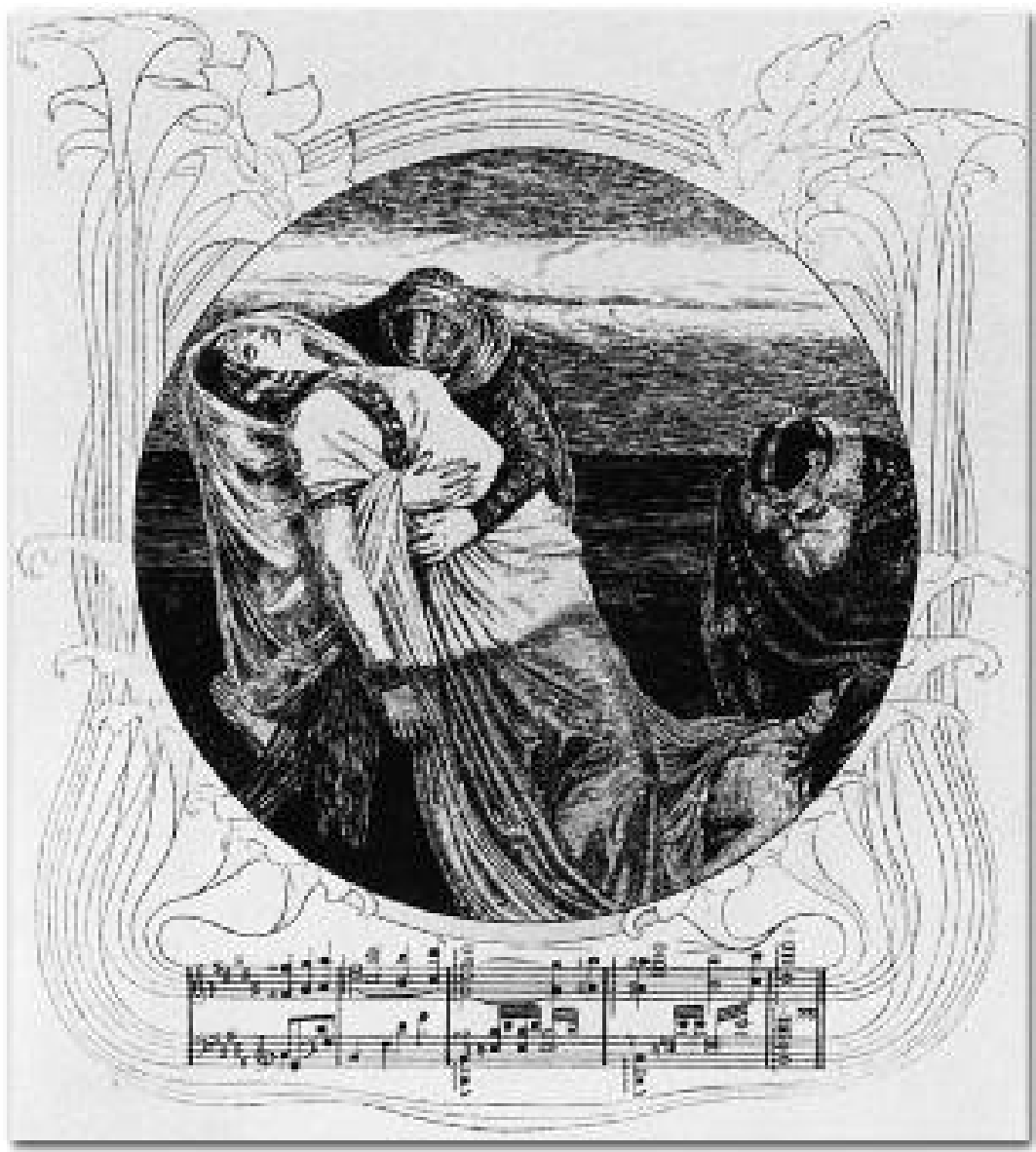

Tristano e Isotta (atto III). Incisione dallo spartito Ricordi, Milano 1907.

l'appunto si possono estrapolare una serie di uguaglianze - Verità $=$ Notte, Musica $=$ Crepuscolo, Arte poetica = Giorno - che associano lo schema delle simbologie del tempo espresso in Tristan (giorno-crepuscolo-notte) a uno schema di drammaturgia traducibile pressappoco così: nel dramma la musica è l'intermediario fondamentale per raggiungere quella verità artistica che si ottiene solo con la fusione perfetta di poesia e musica. Nell'allegoria sessuale dell'ewig ein di Tristano e Isotta s'insinua così anche il corrispettivo più diretto dell'ewig ein poetico-musicale del dramma wagneriano. ${ }^{18}$

${ }^{18}$ Un'efficace interpretazione della teoria wagneriana dei rapporti tra poesia e musica come equivalente del rapporto uomo-donna nei drammi wagneriani (in particolare nel ciclo del Ring) è formulata in JEAN-JACQUES NATTIEZ, Wagner l'androgino. Saggio sull'interpretazione, Torino, Einaudi, 1997 (su Tristan cfr. in particolare le pp. 149-156). 


\section{Guido Paduano \\ La storia d'amore (parafrasi di Tristan und Isolde)}

I

Fa rotta sulla Cornovaglia una splendida nave agli ordini del principe bretone Tristano, con a bordo Isotta, figlia del re d'Irlanda, che Tristano ha chiesto in sposa per conto dello zio Marke, re di Cornovaglia. Questo matrimonio mette fine al conflitto in cui Tristano ha ucciso il guerriero irlandese Morold, promesso sposo di Isotta, che aveva imposto a Marke un feroce tributo.

Dall'alto dell'albero, un marinaio canta una canzone d'amore: amore di terre lontane per una giovane irlandese, rimasta suo malgrado in patria, mentre il vento, sintono ai sospiri di lei, riporta l'amato nella sua.

La canzone fa sobbalzare Isotta come parlasse di lei, che invece suo malgrado naviga verso il paese straniero; l'allusione innocente alla contrapposizione tra i popoli la strappa al torpore assorto in cui si trova e che respinge ai suoi margini il viaggio e la meta: è la sua confidente Brangania a dirle che la navigazione sta per finire, e a nominare la verde terra che le attende. Esplode allora, apparentemente dal trauma dell'estraneità etnica, intesa come sconfitta e prigionia, come vergogna, la spaventevole furia di Isotta: come sua madre ella possiede le arti della magia, ma ridotta alla manipolazione delle erbe, dopo che ha perso l'antico controllo sugli elementi, sul mare, sui venti. Però la distruzione, che non è in suo potere, occupa appassionatamente i suoi desideri: che la nave si spezzi e si inabissi, e vada dispersa ogni vita che essa contiene.

Brangania resta attonita, ma in questa esplosione vede almeno la fine del disperante silenzio di Isotta, che ha lasciato senza un saluto il padre e la madre e ha attraversato il mare fredda, pallida e muta, senza prendere cibo né sonno. Dal candore di Brangania emergono così segnali ambigui, perché associati, per lunga tradizione, non al genere dell'angoscia, ma a quella sua specie che è il disagio amoroso. Lo stesso vale per l'ostinazione nel silenzio, che copre una realtà definita inconfessabile dal codice della virtù femminile. Ma l'ineffabile è detto con altri linguaggi: con la canzone del marinaio che torna a farsi sentire, con lo sguardo di Isotta che immediatamente trova Tristano e resta legato a lui, infine con parole che, provenendo da profondità e autenticità remote, si cristallizzano in un assoluto non comunicabile: «Da me scelto, da me perduto, nobile e sacro, ardito e vile. Capo consacrato alla morte! Cuore consacrato alla morte!».

La coincidenza degli opposti viene subito spiegata: il vincitore di tutte le battaglie teme ed evita lo sguardo di Isotta. 
Come uscire da questa insopportabile solitudine? Perché questo desidera Isotta: il desiderio di morte, balenato nella tempesta universale dei venti e del mare, ma poi specificato nella consacrazione alla morte di Tristano, non è che la soluzione disperata di un desiderio positivo, per quanto siano minacciosamente indefiniti i suoi contorni: avere Tristano per sé, non fosse che appena prima di morire, o per morire insieme.

Per far cessare l'assenza dell'uomo amato, Isotta deve potergliela rimproverare nella lingua degli obblighi sociali. L'artificiosità di questa costruzione si specchia nel fatto che le premesse su cui si fonda erano state rifiutate con ribrezzo da Isotta nelle sue prime parole: chi aveva detto che mai, né oggi né domani, la Cornovaglia l'avrebbe accolta, ora come sposa di re Marke proclama il suo diritto all'attenzione, al riguardo, al servizio cortese di Tristano. «Che pensi tu del servo?», sono le sue parole a Brangania: naturalmente questa designazione resta oscura alla confidente, e Isotta è costretta a specificarla: sentiamo allora squillare in bocca a Brangania un luminoso elogio di Tristano, l'eroe senza pari, quintessenza dei valori, simbolo del mondo fiabesco e prezioso che sta per tramontare di fronte al mondo nudo e feroce della passione.

E infatti Isotta entra sì nell'atmosfera mitica così evocata, ma con ironia sanguinosa, a denunciare l'offesa che le reca il riserbo di lui, e anche ad accennare rapidamente l'altra, più grave: quella di portarla in dono allo zio come un oggetto privo di volontà, una sposa cadavere. Brilla la dignità della persona e della femminilità, ma ne traluce una ben più profonda angoscia per la coartazione della sua vera e nascosta volontà.

Della presunta infrazione alle convenienze Brangania potrà andare a chiedere riparazione, e ordinare a Tristano di presentarsi al servizio di Isotta. A pregarlo di venirti a salutare, suggerisce la conciliante Brangania, solo perché Isotta possa ribadire con ieratica violenza che egli le appartiene, che è la sua signora, la sua padrona. Nella fissità di queste parole, urla l'esigenza controllata del possesso amoroso.

Preannunciata da Kurwenal, il fedelissimo scudiero di Tristano, Brangania è davanti all'eroe e gli comunica che Isotta desidera vederlo. Se è per il lungo disagio del viaggio, risponde Tristano, può rassicurarla: presto finirà. $\mathrm{E}$ a quel punto, al confine tra l'azzurro del mare e il verde dei prati (trema nelle sue parole un carezzevole amore per la sua patria d'adozione), certamente si accosterà a Isotta per accompagnarla e consegnarla al suo sposo. Non prima - quando Brangania insiste perché vada subito da Isotta, il rifiuto si fa esplicito: l'onore le è dovuto in qualunque circostanza (indipendentemente quindi da un contatto diretto), ma Tristano non può abbandonare il timone: la sua vigilanza è l'indispensabile garanzia di un felice arrivo presso re Marke. Nominandolo, l'espressione si dilata in un inchino cortese, che esprime insieme il crisma della regalità e il limpido dovere della fedeltà.

A questo punto, non resta a Brangania che ripetere le imperiose parole con cui Isotta si dichiarava padrona di Tristano. Per Tristano è tanto difficile rispondere che commette una scortesia inattesa: dà il permesso di rispondere al suo posto al ruvido Kurwenal, che è colpito a fondo nella devozione al suo signore e idolo.

Kurwenal nega la qualificazione subalterna di Tristano con argomenti certo non delicati, ma di solare buon senso. Non potrà dirsi servo di Isotta Tristano quando è lui 
che ne fa una regina, è lui che l'ha conquistata e la dona allo zio. L'insinuazione che Isotta non sia dopotutto che una preda di guerra, si sviluppa nella canzone che Kurwenal intona a squarciagola, seguito dalla ciurma: una canzone di guerra in onore di Tristano, che ricorda la sconfitta di Morold.

Perfino Brangania ora è offesa, ma a Isotta importa solo di ciò che ha detto precisamente Tristano: quando le vengono ripetute la sue parole sulla necessità di stare al timone per giungere da re Marke, le ripete una terza volta, pervertendo nel sarcasmo il rispetto dovuto al re, e tornando dolorosamente all'idea di sé come oggetto. Le parti si sono invertite: tocca all'Irlanda pagare tributo, e questo tributo è la sua stessa persona.

Ma se adesso è lei in potere di Tristano, una volta è stato lui in suo potere: su una piccola barca approdò in Irlanda un uomo pallido e malato che si faceva chiamare, per inquieta astuzia, con le sillabe del suo nome disposte in ordine inverso, Tantris: ma la sua malattia era indotta da una ferita infertagli in combattimento, e una tacca nella sua spada corrispondeva a una scheggia nella testa di Morold, mandata da Tristano in Irlanda come lugubre trofeo. Isotta allora volle ucciderlo e alzò su di lui la spada; ma Tristano la guardò negli occhi - lui che adesso evita il suo sguardo - e lei lo risparmiò, curando anzi con le sue arti di maga la ferita. Eterna riconoscenza fu promessa dall'eroe: ma invece egli tornò trasformato da vinto in vincitore, per chiederla in sposa per lo zio, lo stanco re di Cornovaglia. Attraverso l'epiteto passa appena un'ombra dell'antico tema della malmaritata, ma è pretestuoso come tutto ciò che sostituisce la verità ineffabile, per cui invece la colpa di Tristano consiste tutta e sola nel rifiuto di sé, nel mancare di fede al muto impegno dello sguardo, nel far prevalere su di esso le ragioni del mondo, che destinano Isotta ad essere pegno di pace, e lui ad esserne il conquistatore: per questo ruolo Isotta ha pronta una parola orribile: avventura.

Pretestuoso in particolare è il rigurgito di orgoglio nazionale che le fa disprezzare Marke come tributario o vassallo, e dunque indegno della sua mano. Come le ha ricordato Kurwenal, e come lei stessa ha appena detto, proprio le imprese di Tristano hanno ribaltato lo status politico.

Con pazienza Brangania, che in una subitanea illuminazione ricorda di avere lei stessa assistito Tantris, ritesse la tela delle argomentazioni ragionevoli. Come si può accusare Tristano di essere ingrato per aver adempiuto insieme all'obbligo feudale e a quello verso la sua salvatrice, riunendoli in uno solo? In cambio del suo aiuto ha offerto a Isotta un regno su cui egli stesso avrebbe potuto vantare dei diritti, e uno sposo nobile e mite (ecco come si può addolcire il termine "stanco»). Ma Brangania va anche oltre, e troppo oltre: come si può disprezzare Marke, quando il più nobile degli eroi gli è così devoto?

Le ragioni di Brangania smantellano l'alibi politico, e sotto quella corazza scoprono la sostanza dolorante dell'amore, che Isotta, adesso, non può più sottrarsi a comunicare, a meno di rinunciare non solo al dialogo con Brangania e alla consolazione che discende dal riconoscimento della propria infelicità, ma più in generale all'espressione della sua identità: «Non amata, vedere sempre accanto a me il più nobile degli uomini! Come potrei sopportare un simile strazio?» 
Eppure anche di fronte a questa esplicitezza la strategia del tabu trova modo di oscurare il messaggio. Senza nominare Tristano, Isotta ha ripetuto con una minima variazione la perifrasi con cui Brangania lo ha appena designato («il più nobile degli eroi»), ma in Brangania il bisogno di normalità e di pace è così grande da renderla più cieca di quando non ha riconosciuto Tristan/Tantris, e da farle credere che Isotta tema di non avere l'amore di Marke, l'amore canonico e legittimo. Sorride di questa ipotesi, Brangania, orgogliosa del fascino della sua signora come Kurwenal lo è del mito di Tristano: chi potrebbe non amare Isotta? Si potrebbe ammettere solo in un uomo stregato da una magia, ma anche a questo è facile mettere rimedio con i farmaci che la regina d'Irlanda ha donato alla figlia nel partire. Senza questi aiuti, il suo amore materno non l'avrebbe esposta ai rischi di una terra straniera. Sì, risponde amaramente Isotta, ma il farmaco che dà maggior aiuto è quello su cui lei stessa ha inciso un forte segno, il filtro di morte che ha in sé il potere di vendicare il tradimento (che è il nome da lei dato alla presunta unilateralità del suo amore), e dare pace all'angoscia dell'anima.

Mentre Brangania arretra spaventata, si sentono le grida della ciurma che ammaina le vele: la nave sta per approdare. Entra infatti Kurwenal per chiedere a Isotta, da parte di Tristano, di prepararsi ad accompagnarlo incontro al re.

Tristano dunque sembra aver vinto la sua battaglia ed essere riuscito ad avvolgere l'amore di Isotta in un silenzio definitivo, giacché le voci dei marinai scandiscono lo scadere del tempo possibile per un colloquio intimo. Ma proprio la disperazione del momento estremo, la tragicità irrimediabile dell'ipotesi che nulla accada, ribalta la situazione: improvvisamente Isotta decide di sfruttare la vulnerabilità di Tristano alle ragioni politiche che si risolvono nella lealtà verso Marke; la conosce bene perché ne è stata già vittima, quando l'uomo da lei salvato si è ripresentato a trattare la pace. In base a questo codice, nessun altro che Tristano può accompagnare Isotta davanti al re: lui stesso, del resto, ha fatto parola a Brangania di questa imprescindibilità. Qui dunque Tristano può essere colpito (e si può anche dire ricattato): Isotta non camminerà al fianco di Tristano se prima Tristano non otterrà il suo perdono per una colpa non espiata. Kurwenal, che in Isotta vede solo uno smodato orgoglio, annuncia seccamente che riferirà il messaggio.

Rimasta sola con Brangania, Isotta prorompe in un addio al mondo e ai genitori che lascia Brangania attonita e incredula. Isotta è decisa a usare il filtro di morte su se stessa, oltre che sul traditore Tristano, e con amara ironia ritorce le parole di Brangania: sì, sua madre ha ben provveduto ai rischi di una terra straniera, si deve esserle grati per il farmaco che cura la più profonda sofferenza, il più alto dolore. Lo prepari dunque Brangania, in nome della sua fedeltà.

Annunciato da Kurwenal, entra Tristano, ma passa un lungo tempo prima che il dialogo inizi, lasciando l'impressione che lui e Isotta saggino il terreno e prendano posizione come per un duello - discorsivo, ma mortale, e finalmente diretto, dopo le avvisaglie e le mediazioni della doppia ambasceria.

Parla per primo Tristano, con le parole apparentemente neutre e minimali della cortesia: «Ditemi, signora, che cosa desiderate». Apparentemente, perché l'appellativo di 
«signora» è una volta per tutte uscito dal codice con l'uso esasperato che ne ha fatto prima Isotta; inoltre Tristano, ignorando la parte specifica del messaggio di Kurwenal, quella relativa all'espiazione, dà l'impressione di rispondere, tardivamente, alla prima convocazione di Isotta. Ciò lo mette in una posizione di debolezza di cui Isotta approfitta subito, rimproverandogli adesso di persona la poca attenzione usata verso di lei. Responsabile di ciò, risponde Tristano, è il senso dell'onore e l'obbedienza verso il re, e all'incredulità ironica di Isotta (come è possibile che l'obbligo di onorare il re si risolva nel rifiutare obbedienza alla regina?), esplicita il dettato socio-antropologico: «il costume del mio paese insegna che durante il viaggio nuziale chi ha condotto la trattative di nozze si deve tenere lontano dalla sposa».

Perché, per quale preoccupazione? Ovvero, che cosa si teme che succeda? La domanda bruciante e impudica di Isotta esplode accanto a Tristano, che evita il passaggio al dialogo intimo, ricorrendo all'autoreferenzialità del codice: "Chiedetelo al costume».

Su questo punto Isotta non può che dichiararsi sconfitta, ma sul terreno di questa provvisoria vittoria, Tristano ha lasciato la coerenza della sua argomentazione: più nessuna traccia dell'obbligo di tenere ininterrottamente il timone! Le conseguenze di questa dimenticanza sono radicali: se le ragioni addotte sono dalla loro stessa varietà ridimensionate a pretesti, si apre uno spiraglio in direzione di un'autenticità di Tristano, anche nel suo caso opposta al significato superficiale delle sue parole, e destinata piuttosto a inverare il misterioso sguardo che un tempo ha alzato sugli occhi di Isotta. In questa luce, proprio l'ostinata resistenza di Tristano a mettersi nelle condizioni formali di recepire il messaggio di Isotta diviene prova della sua impossibilità a resistere alla sostanza del messaggio.

In ogni caso, la vittoria del codice sociologico permette alla pur sconfitta Isotta di addurlo anche a proprio vantaggio, aggrappandosi ancora al mascheramento offerto dal contenzioso politico, ovvero dal fantasma, ricorrente solo a questa finalità, della nazionalità offesa: costume infatti altrettanto inveterato è la conciliazione con un nemico tramite l'espiazione dell'offesa. Quale nemico, chiede, stavolta ingenuamente, Tristano e, come aveva fatto già prima Brangania, ricorda che si è celebrata una solenne conciliazione pubblica. Ma quella non conta per Isotta: in quella è stata promessa a Marke come una sposa cadavere e, conseguentemente, non si è ritenuta coinvolta in nessun impegno: ma c'è una vendetta privata che non è stata compiuta, quella di Morold giurata dalla sua promessa, a quanto ella stessa dice. Proprio questa riserva fa Tristano: tocca a lui ora infatti rivolgere una domanda impetuosa, provocante e impudica: davvero v'importa di questo? Anche in questo caso demistificare il pretesto indica una via per penetrare nell'abisso della causa vera. Ma la principessa irlandese non può che sostenere il suo pretesto: "Osi schernirmi? A me era promesso, il nobile eroe irlandese, io avevo benedetto le sue armi, nel mio nome entrava in battaglia. Quando egli cadde, cadde il mio onore». È lei adesso ad adottare il tono della mitologia cortese e fiabesca che prima, a proposito di Tristano e di Marke, usava solo con sarcasmo: anche a lei dunque il mondo preesistente all'amore era caro, ma la strumentalizzazione che ne viene fatta riverbera su di esso una nostalgia inattuale. E comunque la promessa, l'onore, 
la benedizione delle armi, le insegne: non è d'amore che si parla, la finzione non si spinge a nominare invano l'ineffabile.

Come finzione questa si rivela implacabilmente quando deve integrare nel sistema argomentativo un resoconto dell'incontro fra Isotta e Tantris differente da quello fornito a Brangania, che è inconfutabile come tutto ciò che sulla scena viene comunicato ai confidenti; ma la nuova versione è funzionale a stabilire la necessità dell'espiazione, e la contorsione didascalica del discorso marca penosamente la funzionalità: "Malato e stanco, in mio potere, perché non ti ho ucciso? Posso risponderti facilmente». Non per ciò che solo l'eufemismo obbligava, nel dialogo con Brangania, a chiamare "pietà», Isotta ha risparmiato Tristano, ma per un barocco perfezionismo della cavalleria: il nemico doveva essere perfettamente guarito perché venisse consumata su di lui la vendetta in combattimento. Ma nel frattempo l'abilità diplomatica di Tristano, quella stessa che ha fatto di lei una sposa cadavere, gli ha conquistata l'amicizia di tutti: chi dunque adesso deve colpire Tristano?

Tristano non sfugge alla strettoia logica: deve colpire Tristano la sola persona estranea al patto della conciliazione: «Se Morold ti era tanto caro, allora riprendi in mano la spada, e vibrala con mano forte e salda, in modo da non farla cadere!»

L'insieme delle affermazioni di Isotta rende chiaro tuttavia che la sua estraneità alla conciliazione non concerne il permanere in lei del desiderio di vendetta, ma l'inconciliabilità dell'amore respinto. E questo chiaro anche a Tristano? Sì: un solo grandioso particolare lo indica. Le sue parole seguono con precisione ineccepibile il percorso apparente di Isotta: ma improvvisamente, in questo momento e per la prima volta, le si rivolge con il tu: c'è in questo una verità più forte di ogni contenuto mistificatorio, ed è questo a mostrare sincera la sua offerta della vita, che di conseguenza va letta non come acquiescenza a una vendetta tutta esterna, ma come dono d'amore: il primo, l'ultimo, il solo possibile. Sono dunque già vicinissimi Tristano e Isotta, quanto quest'offerta è vicina alla morte data col veleno come violenza amorosa.

Senza il tu, sarebbe legittimo pensare che Tristano porti alle estreme conseguenze l'architettura di Isotta proprio per scoprirne la mistificazione (volgarmente parlando, che sia andato a 'vedere' il suo bluff). Questa è infatti proprio la sensazione di Isotta, che si sente prigioniera del suo discorso e ne esce con una fatica tortuosa, simile a quella necessaria per costruirlo; uccidendo Tristano non solo offenderebbe Marke, il suo sposo, ma ancor più offenderebbe se stessa, accettando di essere sottovalutata: uccidere Tristano, infatti, vorrebbe dire negare l'importanza del dono che lui solo era in grado di portargli, quello che ha scelto con uno sguardo misuratore, posato sul suo corpo ad affermare sì un possesso, ma il possesso gelido e interlocutorio di un sensale. Isotta parla dello sguardo che le ha rivolto Tantris quando lei ha alzato la spada per ucciderlo, lo stesso sguardo che lei stessa ha posto all'origine del suo amore. In questa velenosa ambiguità sta la possibilità di capire perché Isotta considera Tristano un traditore.

Brangania ha preparato il filtro; altre grida dei marinai fanno intendere che l'approdo sta per avvenire: lo avverte Isotta, disperatamente vigile, prima di Tristano, assorto 

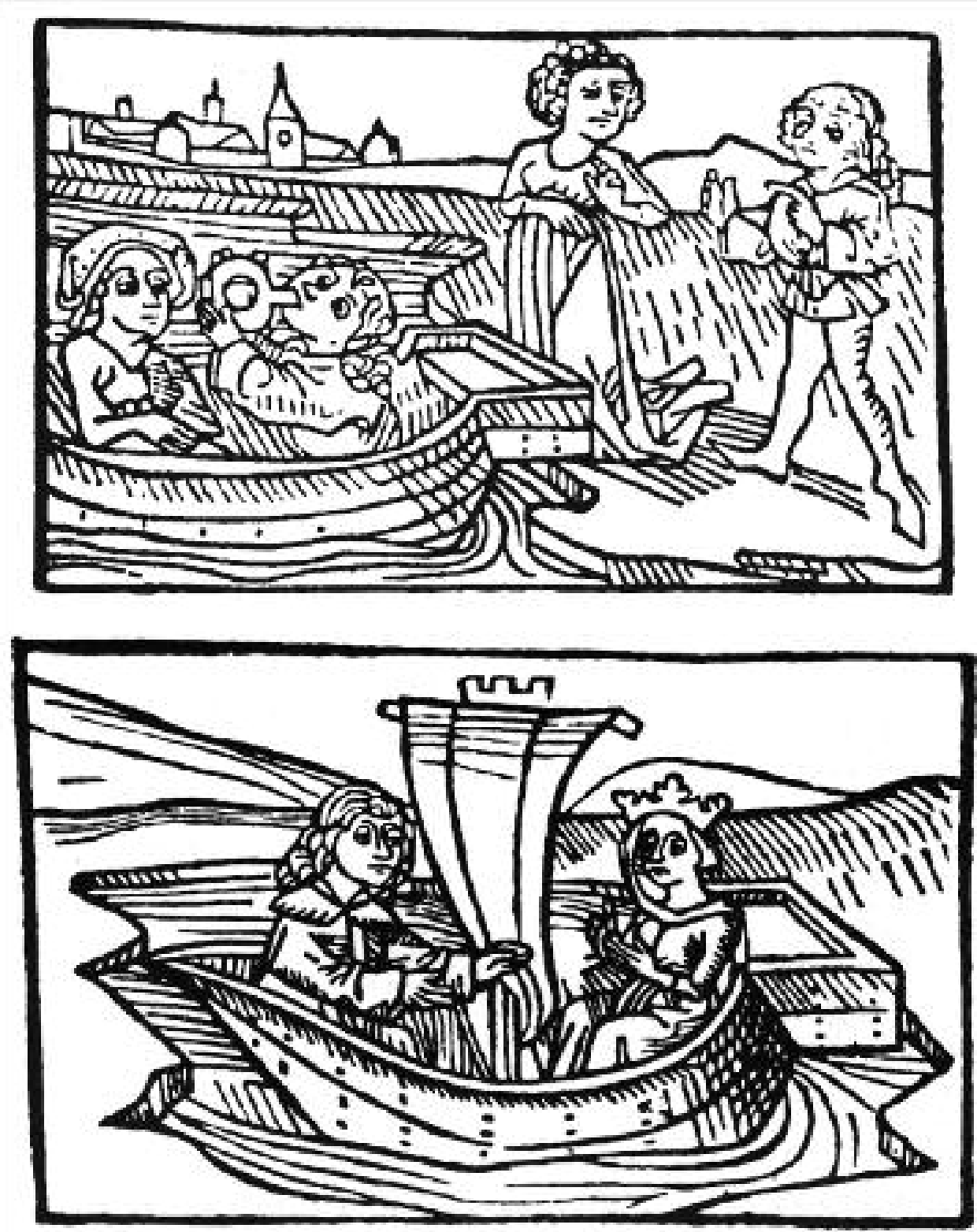

Tristano beve il filtro magico e L'arrivo di Isotta a Kareol, xilografie da Hienach volget die Histori von Herren Tristrant und der schönen Isalden von Irlannde, Augsburg, Anton Sorg, 1484 (rist. anastatica Hildesheim, Olms, 1989), versione in prosa del romanzo in versi Tristrant di Eilhart von Oberg (sec. XII). 
nello stesso oscuro altrove in cui Isotta è apparsa all'inizio. Capisce quello che Isotta gli ha taciuto, la verità amorosa sotto la maschera dell'ostilità; tace a sua volta quello che lei non ha capito, che la sua rinuncia a lei è anch'essa e da sempre atto d'amore. Vero è che la sua verità è sepolta ben più profondamente di quella che traspare da Isotta con commovente immediatezza; e però, non abbastanza profondamente.

Quando Isotta gli offre la coppa, tornando con lo strazio ossessivo dell'ironia a descrivere l'offerta cerimoniale e oggettuale della sua persona a re Marke, Tristano gliela strappa di mano: in bocca a lui, gli ultimi ordini alla ciurma, che segnano la consumazione del suo ruolo sociale e il congedo da esso, precedono un giuramento che attesta l'onore e la fedeltà al suo re, ma insieme l'infelicità, la coscienza della resistenza attuata contro se stesso e dell'inganno portato al suo proprio cuore, e l'accettazione consapevole del veleno come rimedio: l'ultimo farmaco che gli viene dalla regina dell'Irlanda per la sua guarigione piena.

Ma Isotta gli strappa a sua volta la coppa: berla da solo sarebbe un nuovo tradimento, dopo la separatezza che Tristano ha creato con la riconciliazione pubblica, e applicato nell'incubo sordo del viaggio.

Passano lunghi attimi nell'attesa consapevole della fine. Ma la fine non sopravverrà, perché l'affetto di Brangania ha supplito al principio di conservazione che in Isotta è stato ucciso dall'amore, e nella precipitazione del gesto irreversibile ha scambiato il filtro di morte con quello d'amore. È questo dunque che opera, ma l'effetto è soltanto quello di estrarre da entrambi il solo aspetto dell'amore ancora ignoto, la tenerezza immediata, le parole equivalenti a carezze, "caro", "cara»: in esse si addolcisce, permanendo, il rimprovero di Isotta, si libera il silenzio di Tristano. Identico, armonioso, unisonico è il linguaggio dei corpi.

Ma Brangania è subito sbigottita e pentita: la lunga sofferenza che germoglierà dal suo atto le fa rimpiangere la morte rapida che ha impedito.

Dal loro mondo esclusivo, inopinatamente raggiunto, con terribile ironia, proprio mentre raggiungono il mondo sociale, Tristano e Isotta guardano indietro al penoso itinerario dei fantasmi sconfitti: l'onore di Tristano, la vergogna di Isotta. Respinti insieme nello stesso limbo, benché la vergogna di Isotta sia sempre stata inautentica, e l'onore di Tristano sempre autentico, ma all'inautenticità lo fa regredire l'autenticità superiore dell'amore.

Eppure una differenza si riproduce nell'ambito pragmatico, più angosciosa che mai: mentre l'Irlanda è definitivamente alle spalle di Isotta, l'universo degli obblighi di Tristano preme dalla riva e dal castello che corona la scogliera, e preme tanto più rischiosamente quanto più Tristano lo disconosce: quando Kurwenal gli annuncia l'arrivo del re, ansioso di incontrare la sua sposa, la risposta di Tristano, fortunatamente quasi dispersa dall'esultanza collettiva, è "Quale re?»: il sacro legame di fedeltà, l'asse portante dell'agire, non è solo sconfitto, è precipitato nell'ignoto e nell'assurdo.

Ma ha appena il tempo Brangania di spiegare ad Isotta che cosa è successo, mentre la avvolge nel mantello reale e tutt'intorno squillano le trombe a ribadire la saldezza della regalità e del sistema imperniato su di essa, che torna a Tristano la coscienza del 
conflitto, che lo fa uscire dall'istante di felicità incondizionata: l'inadempienza degli obblighi e l'inganno sono all'origine della sua gioia.

Nelle stanze della regina, Isotta e Brangania sono sole: il re e il suo seguito sono appena partiti per una battuta di caccia; i loro corni echeggiano allontanandosi. Abbastanza lontani li giudica Isotta perché a Tristano possa venir dato il segnale di accorrere da lei, ma non è di questo parere la prudente Brangania: suono e distanza sono in funzione dei loro stati d'animo, che a vicenda si rimproverano. Per Isotta, Brangania si fa ingannare dalla paura, fino a sentire il segnale d'allarme dove è soltanto la voce amichevole degli elementi, il riso del vento sulle fronde, il mormorio della fonte. Per Brangania, Isotta ingloba anche la natura nell'avidità della sua passione.

Ma le sue preoccupazioni vanno ben oltre il calcolo del tempo che può garantire la tranquillità al convegno amoroso: teme piuttosto che la relazione fra Tristano e Isotta non sia ignota a tutti come Isotta confida. Certo l'agitazione della sposa al momento dell'arrivo è stata attribuita dal buon re Marke ai disagi del viaggio; ma c'è qualcuno che da allora sospetta e li spia, Melot.

Più che mai su questo dissente Isotta: certo che Melot sa, ma essendo buon amico di Tristano favorisce il loro amore; è stato lui a progettare la caccia che Brangania sospetta invece essere una trappola. Con la stessa perentorietà con cui proclama quest'amicizia, Isotta ordina a Brangania di spegnere la lampada, solo segnale di luce nella notte che già avvolge i cuori in un brivido di piacere. Di nuovo Brangania si sottrae: la lampada è utile a mettere in guardia dal pericolo che incombe costantemente su di loro, da quando lei stessa ha preparato per la sua padrona il disonore, anziché la morte che le era stata richiesta. Ma Isotta la libera da ogni responsabilità: in quella circostanza è stato l'amore stesso, la Minne, ad affermare la sua sovranità su vita e morte, e sulla persona che arbitrariamente aveva scelto la morte. A Brangania che ripete il suo invito alla prudenza, Isotta ripete la forza imperativa dell'amore che risplende nella notte, non tollerando altre luci; il suo ordine deve essere eseguito anche a costo che la lampada spenta configuri lo spegnimento della vita. Dopo avere accettato la morte come esito disperato dell'amore, Isotta accetta adesso la morte come rischio della scommessa amorosa. Sconfitta nelle sue richieste, Brangania manifesterà la sua fedeltà stando di guardia.

Al lume spento, e al cenno che Isotta fa con un fazzoletto, entra Tristano. L'incontro tra i due amanti è un crescendo inarrestabile di passione, di appropriamento, di certezza, di splendore dei sensi, di idoleggiamento del corpo, di aspirazione all'unità. Con tenero sollievo riappare in questa prospettiva il vicino passato della loro lontananza: Tristano lamenta di avere a lungo aspettato il calare del sole, segno nemico, segno di impossibilità e separatezza, e quando finalmente il sole è calato, una luce artificiale si è assunta gli stessi compiti, finché Isotta l'ha spenta, sfidando nel giorno, più ancora che il rischio temuto da Brangania la pertinace negazione dell'amore. 
Sul giorno si avventa l'odio di Tristano: vorrebbe poterlo spegnere in tutta la sua vasta capacità di provocare sofferenza, con la stessa semplicità del gesto con cui Isotta ha spento la lampada - tardi tuttavia rispetto all'ansia del desiderio.

Ben altro ritardo, tuttavia, può Isotta, con ferma tenerezza, rimproverare a Tristano: nelle sue parole, assieme all'ampiezza simbolica del giorno si estende la sua demonizzazione, fondata sulla rievocazione delle vicende precedenti al filtro di Brangania. La luce respingente del giorno rappresenta l'insieme degli ostacoli posti alla libertà amorosa, e dunque, per una metafora che irrompe a questo punto nel dialogo con valore fondante, l'insieme delle relazioni mondane che con la loro stessa esistenza escludono la solitudine a due. In testa a tutte, per valore significativo e per concreta nocività, la relazione di dipendenza e fedeltà fra Tristano e Marke, in nome della quale è stato stabilito che non per sé ma per lo zio Tristano chiedesse Isotta in sposa.

Sì, conferma Tristano: ma una forma del giorno era anche la bellezza stessa di Isotta, e l'onore pubblicamente e universalmente a lei tributato, che gliela facevano sentire inattingibile: «nella chiara luce del giorno, come poteva Isotta essere mia?»

Isotta nega: lei era sua per il solo fatto di averlo scelto. Meno condizionabile dalle esigenze del mondo, su cui fa prevalere la propria volontà, Isotta è, proprio per questo, meno radicale nel rifiutarle. Si capovolge così la presentazione iniziale, con l'antitesi fra la perfetta cortesia di Tristano e la selvaggia incomunicabilità di Isotta: solo circostanziale questa seconda, mascheramento della scissione interiore la prima.

La vicenda di questa scissione Tristano ora la racconta con un ritmo ansioso, esasperando la contraddizione per cui Isotta gli si è insieme presentata come icona gloriosa del mondo e come portatrice di un ideale segreto, maturato nella profondità inconscia del suo io, caro proprio perché inattingibile. Inoltre, rivela che il mondo sociale ha agito su di lui non solo nella forma positiva della fedeltà verso lo zio, ma anche nell'invidia, nella diffidenza, nella meschinità che hanno esercitato su di lui un'azione provocatrice, determinando in risposta il suo sacrificio.

Nella sua risposta, Isotta rivive la sofferenza bruciante che il sacrificio di Tristano le ha procurato, e che, in forma poco meno esplicita, già prima del filtro aveva manifestato: esplicita ora diventa soltanto la compresenza, o anche l'identità, fra l'amore e l'odio che nutriva per lui. Vengono rievocati il riserbo di Tristano nel sottrarsi a Isotta, e l'arma con cui Isotta l'ha contrastato e che adesso viene reinterpretata nei termini dell'opposizione notte/giorno: Isotta rivendica infatti l'adozione del veleno come negazione del giorno 'traditore', come ingresso nello spazio notturno privo di inganni, sede di un amore eterno, fondato e consacrato dalla morte comune. Tristano conferma, e conferma anche di avere con il medesimo spirito accolto il presunto veleno. Che fosse invece il filtro amoroso ha rilievo soltanto - e non è poco - per il fatto che il legame amoroso, comunque eternamente stabilito in quell'attimo, può o deve manifestarsi sulla terra, nella sopravvivenza empirica delle due persone.

Il dialogo tra Tristano e Isotta affronta infatti a questo punto e a questo proposito la sua fase più ricca e sofferta, mettendo in discussione due antitesi che possono, o no, essere intese come equivalenti: quella tra vita e morte e quella tra giorno e notte. 
Certo, quando la disperata Isotta ha voluto uccidere e morire, questa sembrava la sola possibile scelta amorosa, e dunque le due opposizioni erano forzatamente identiche; quando il filtro d'amore fa sì che Tristano sopravviva manifestando il suo profondissimo e nascosto amore, le opposizioni si divaricano, perché al giorno, comunque sconfitto, si oppone una realtà vittoriosa che non è più la morte, ma appunto l'amore, la notte d'amore. In altre parole, mentre la schiavitù di Tristano nei confronti del giorno riduceva ogni esistenza possibile alla vita sociale, il filtro di Brangania, o meglio la volontà della Minne - mi spingerei perfino a dire il suo principio di autoconservazione - apre il conflitto tra due forme di esistenza, il vivere sociale e il vivere notturno.

In questo senso, proprio Tristano corregge Isotta, la quale sembra rammaricarsi del fatto che l'inganno di Brangania abbia restituito Tristano al giorno: al contrario, «attraverso la porta della morte, dove è fluito per me ampio e ricco, mi ha aperto ciò che avevo sperimentato solo in sogno, il regno meraviglioso delle notte». Così la luce del giorno è svanita.

Non del tutto, ribatte impetuosamente Isotta, così come la loro vita d'amore non è tutta la vita, ma un tempo ansiosamente strappato alla situazione che Tristano ha creato: è la regalità di Marke la forma predominante del giorno, e ad essa Isotta è stata donata: «Come ho potuto sopportarlo, come posso, ancora adesso, sopportarlo?».

Perché, risponde Tristano, la totalità amorosa risiede nell'interiorità: ciò che importa è che l'astuzia invidiosa del mondo può sì separarli (materialmente), ma non più ingannarli. Chi è consacrato alla notte, lo è una volta per tutte, e i fascini del giorno, la gloria, l'onore, il potere, non hanno più presa su di lui: la sua presenza nel giorno non è che attesa e desiderio della notte, dove gli sorride, primaria ed eterna, lei sola vera, la gioia d'amore.

L'invocazione alla notte avvolge ora insieme Tristano e Isotta, che le chiedono di scendere su di loro e accoglierli nel suo grembo, di sciogliere nel suo sacro crepuscolo l'inganno del giorno. Allo splendore del sole succedono le stelle del piacere, mentre l'incanto notturno palpita nel contatto dei corpi - dei cuori, delle bocche, del respiro.

Così si raggiunge la certezza che il mondo non esiste come realtà distinta e contrapposta al soggetto amante, questa contrapposizione essendo appunto il capitale inganno del giorno; il mondo coincide con la persona dell'amante, o meglio con le due persone unite nell'amore e nell'unisono: sta in loro tutta la vitalità e il palpito dell'universo, raccolti nel desiderio autentico e consapevole di non svegliarsi mai più (cioè di non concedersi mai più al giorno).

Su questa professione si eleva lontana e quasi ironica la voce di Brangania dalla sua guardia sulla torre: la notte sta per tramontare, è necessaria l'attenzione al giorno e ai suoi pericoli, ai rapporti sociali tanto compromessi.

A Isotta che pur lievemente lo invita a prestare ascolto, Tristano sussurra: «Lasciami morire». Di nuovo la notte si identifica con la tentazione della morte, se la vita li chiama così perentoriamente al risveglio ed al giorno.

$\mathrm{Ma}$ a questa tentazione Isotta si sottrae con una resistenza commovente, investendo di volontà positiva il margine problematico della distinzione tra i vicini universi della not- 
te e della morte, e dilatandolo fino a fare della morte l'altra nemica dell'amore, oltre al giorno. Dall'una e dall'altro partono infatti aggressioni simmetriche e complementari all'amore: mentre il giorno ne attacca i valori (cui è puntualmente avverso), la morte ne attacca la sussistenza empirica, avvolgendolo nella sua vanificazione universale.

Non è così per Tristano, il quale sostiene che la morte non rappresenta una vera minaccia per l'amore. Per il corpo, per la vita sì, ma l'amore non può morire assieme ad essi, perché è eterno e dunque immortale. Ma se non può morire l'amore, nemmeno Tristano può morire, cioè venir meno all'amore, che rappresenta la sostanza del suo essere.

Isotta non si lascia persuadere: per quanto l'amore sia una forza eterna, esso si realizza nel legame temporale tra due esistenze limitate, legame rappresentato dalla soave e fragile congiunzione «e», che associa i loro nomi, ma anche la concreta presenza dei loro corpi. Esso non potrebbe che scomparire assieme alla vita delle persone.

No, ribatte Tristano, non la congiunzione sarebbe vanificata, ma al contrario le imperfezioni e i limiti che adesso la vita empirica pone alla totalità dell'amore. Il corpo è dunque visto come la sede della limitazione e della ricattabilità umana (nella fattispecie rispetto alle esigenze mondane rappresentate dal giorno), piuttosto che come quel meraviglioso strumento di comunicazione amorosa che pure sta vibrando nell'intensità del loro dialogo.

Isotta ancora obietta: la domanda paradossale di Tristano (se l'amore è immortale, come può morire la persona dedicata interamente all'amore?) ha secondo lei una semplice risposta: con la morte della persona amata.

E tuttavia questa prospettiva della fine comune ha così grande dolcezza, e così grande maturazione rispetto al medesimo progetto che selvaggiamente Isotta aveva formulato utilizzando il filtro di morte, che si tramuta senza soluzione di continuità in una fantasia idoleggiata. Implicitamente, nel linguaggio intenso dei gesti, anche Isotta accetta l'idea che la morte sia la rimozione delle impurità e il compimento perfetto dell'amore.

La morte anzi è già un calmo possesso, che si può addirittura collocare nel passato, e in tal modo conferisce carattere definitivo alla precarietà che la vita amorosa condivide con qualunque altra forma di vita: «Così morimmo senz'essere separati, per l'eternità una cosa sola, senza fine, senza risveglio, senza angoscia, senza nome, abbracciati nell'amore, totalmente devoti a noi stessi, vivendo soltanto per l'amore».

Fedelmente Isotta riprende una per una queste parole, tranne proprio all'inizio: "Così morremmo", dice, anziché "così morimmo». Per lei l'iniziazione alla morte è ancora una prospettiva ipotetica, da cui la separa un tenerissimo brivido.

Ma appena di nuovo risuona in lontananza la voce di Brangania a chiedere attenzione e a prospettare i limiti della notte, le parti si invertono rispetto a prima: è Isotta a invocare la morte contro quei limiti, e a chiedere che «il giorno ceda alla morte» - la stessa frase che prima aveva sollevato le sue obiezioni e aperto il grande dibattito.

Al posto di esso, c'è ora una nuova invocazione alla notte d'amore, dolce ed eterna, che impercettibilmente trasmette la propria mitologia alla morte: morte bella, morte d'amore. La morte che non ispira più timore, ma ospita il calore antico e primigenio, libero dall'angoscia del risveglio, il luogo della fida solitudine a due, l'eternità di un fo- 
colare domestico dilatato in spazi smisurati: qui per Tristano Isotta non è più Isotta, è Tristano, e Isotta a sua volta si rivolge a Tristano chiamandolo non più Tristano, ma Isotta. Rinunciare al proprio nome, distintivo e parziale, è abolire coscientemente ogni residuo di separazione: ma mentre nello sfavillare della soggettività attraverso l'alterità arriva a compimento la vicenda amorosa, precipita improvvisamente sulla scena il risveglio del mondo.

Si ode prima il grido tardivo di Brangania, poi quello di Kurwenal che all'ultimo momento la sostituisce nella guardia, appena prima che entrino Marke e il suo seguito. In un silenzio spettrale albeggia, e Tristano accoglie per l'ultima volta il livido giorno - ugualmente nel suo aspetto astronomico e nelle sue valenze simboliche.

È Melot, sul conto del quale non si era ingannata Brangania, a rivendicare la veridicità della denuncia che ha avanzato contro Tristano, mettendo in pegno la sua testa, e si vanta di avere salvaguardato il nome e l'onore del re. Risponde Marke, con infinita amarezza, che ben diversamente stanno le cose: il tradimento di Tristano, che con il suo atto più libero, la sua scelta più essenziale, l'ha colpito a morte, è una privazione affettiva che nessuno può riparare, tanto meno Melot - di cui Marke si vendica con il disprezzo aristocratico e implicito che gli spetta per avere a sua volta tradito uno dei frastagliati e contraddittori obblighi feudali, l'amicizia per Tristano.

Questo e gli altri fantasmi del giorno Tristano li esorcizza ed espelle tutti con violenza dall'universo segreto. Ma non è facile farlo rispetto a Marke che gli chiede conto della sua relazione con lui: mondana sì, ma tanto profonda da avere a lungo fronteggiato, e addirittura apparentemente prevalso sull'amore.

Per Marke non c'è più al mondo né fedeltà né onore né virtù, se non si possono più ritrovare in Tristano: la storia dei suoi passati servigi si riduce all'assurdo, se essi devono venire ripagati dal disonore del re. Eppure Marke aveva mostrato la sua riconoscenza facendo di Tristano il suo erede e trattandolo, una volta rimasto vedovo senza figli, come un figlio. Proprio per salvaguardare la sua eredità, Marke non avrebbe voluto risposarsi, ma aveva ceduto alle pressioni della corte e del paese, e soprattutto del medesimo Tristano, che minacciò lo zio di andarsene per sempre dalla Cornovaglia se non avesse ricevuto l'incarico di andare lui stesso a procurargli una sposa: la donna meravigliosa in cui si rintraccia la quintessenza della felicità, e alla quale Marke mai avrebbe osato aspirare.

Questo comportamento di Tristano sembra a posteriori ispirato alla crudeltà più feroce: la felicità che egli ha procurato allo zio si risolve in un maggior rischio di perdita e di dolore - e lui stesso ha procurato entrambi allo zio. Non solo: ha seminato in lui il veleno del sospetto, ha inquinato la sua fiducia, costringendolo a tendergli un umiliante agguato, il successo del quale si identifica con l'infelicità e il disonore. C'è in questa massima divaricazione dei suoi comportamenti un mistero inesplicabile.

Tristano annuisce: ai perché del re non c'è risposta; lo straziato, superstite affetto nei suoi confronti si esprime solo nel tono tenero che assume il rifiuto di rispondere.

Ma il lamento di Marke ha fatto rivedere da una nuova prospettiva il travagliato iter, già noto dal racconto di Tristano, che ha portato l'eroe in Irlanda a chiedere la mano di 
Isotta: lo scontro tra ragioni del giorno e interiorità notturna fa ora intravedere un lucido accanimento suicida, un percorso verso l'annientamento intrapreso soffocando l'amore e confinandolo nella zona ineffabile, dove Tristano lo ha mantenuto fino alla fine del viaggio per nave: un percorso non essenzialmente diverso da quello che porta l'amore, una volta esplicitato, a cercare nella sparizione fisica il suo compimento.

Tristano infatti si rivolge a Isotta, invitandola a seguirlo nella sua terra: lo spazio oscuro, primario, prenatale, che abbandonò per venire alla luce, quando sua madre morì nel partorirlo. Questa richiesta ha il carattere formale di una proposta di matrimonio, e negli stessi termini risponde Isotta, contrapponendola alla richiesta, fattale un tempo, di andare in una terra straniera, sposa di un estraneo (che dolorosamente la ascolta). Allora obbedì a forza; ora che Tristano la richiede per sé e per la sua terra, che comprende tutti i mondi, e che in quella stessa notte essa ha accettata come propria, il suo assenso è pieno, e lietamente riprende l'antica formula matrimoniale: dove è la casa e il focolare di Tristano, là sarà Isotta.

Questa castissima cerimonialità suscita il furore di Melot, che incita il re a vendicarsi. Con amare parole Tristano commenta il comportamento dell'uomo che un tempo era suo amico, e che è stato abbagliato a sua volta dalla bellezza di Isotta: avendo agito per gelosia, Melot non è il restauratore dei valori sociali offesi, ma, appunto, il traditore di un traditore.

Questa accusa è accompagnata dal gesto con cui Tristano sfida a duello Melot; ma appena questi sguaina la spada, Tristano se ne lascia trafiggere, lasciando cadere la sua.

III

Gravemente ferito, ma vivo, Tristano è stato trasportato da Kurwenal nel fatiscente castello dei suoi avi, a Kareol in Bretagna. Ora è sprofondato in una specie di coma o trance, e Kurwenal e un pastore, anch'egli fedelissimo, aspettano che si risvegli; ma Kurwenal teme che il risveglio di Tristano coincida con la sua morte, e ripone ogni superstite fiducia nell'arrivo di Isotta. Per questo il pastore è stato mandato di vedetta: il suono ineffabilmente triste della sua zampogna segnala appunto una risposta negativa, sia pure provvisoriamente negativa.

E questo suono a destare Tristano con la voce di una remota familiarità: «l'antica melodia!». Kurwenal è invaso da immensa gioia, e infaticabilmente risponde alle lente domande di Tristano, per quanto esse testimonino disagio, pena e fatica del riadattarsi all'esistere. Il mondo, che lo ha richiamato a sé, gli sfugge nella nettezza dei suoi confini: lo sbigottiscono il richiamo ai suoi antenati e ai suoi beni - che inutilmente Kurwenal tenta di riportargli alla memoria, ricordandogli che ha donato il suo castello al popolo quando partì per la Cornovaglia. Per l'ultima volta la citazione delle sue imprese eroiche prende il tono trionfale dell'inno.

Tristano chiede allora se si trova in Cornovaglia: è dunque in grado di afferrare singoli nuclei semantici collegati a singole parole, non la dimensione sintattica del discorso e neppure dunque la diacronia temporale che essa riflette. Pur scoraggiato, Kurwe- 


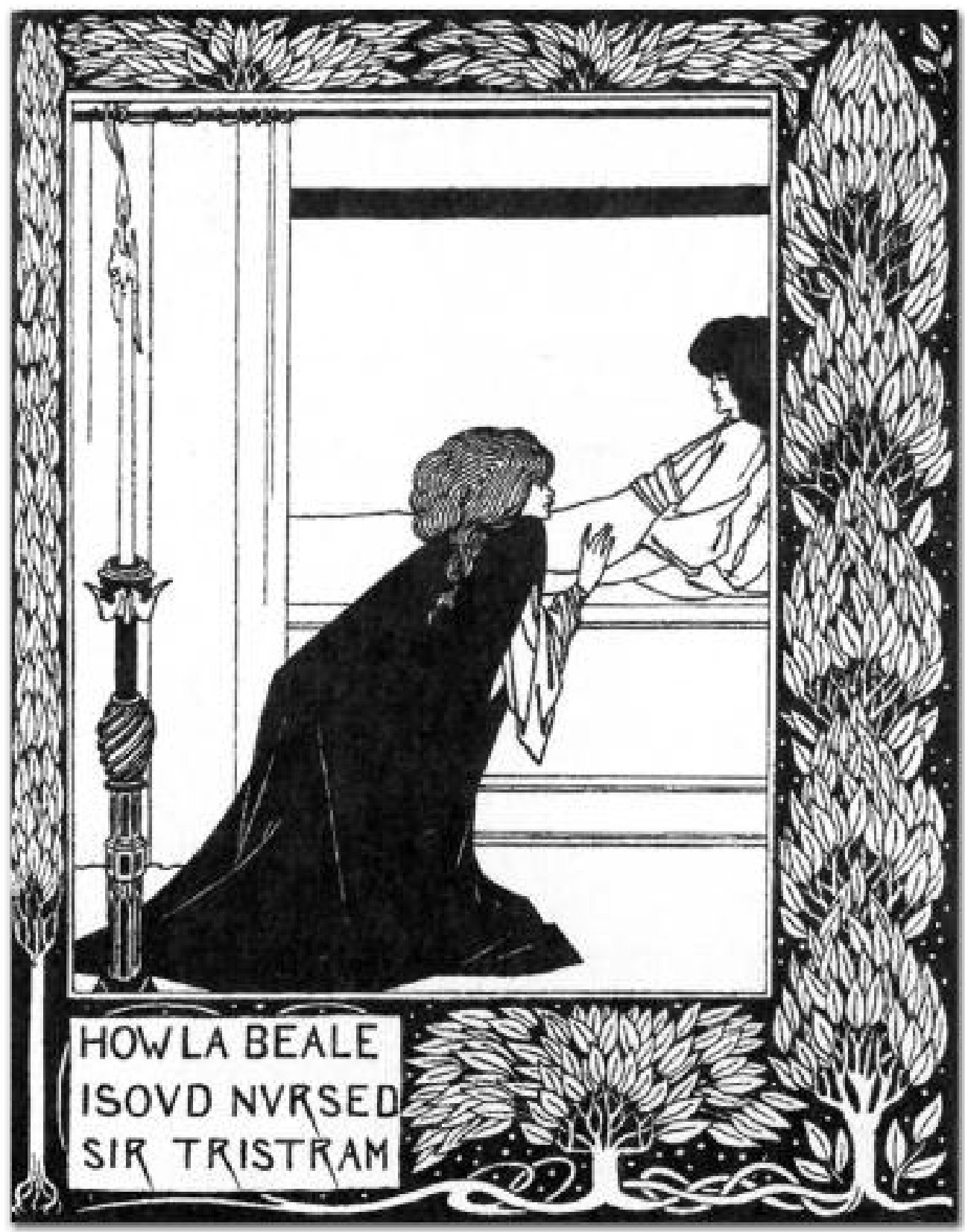

Aubrey Beardsley (1872-1898), How La Beale Isoud Nursed Sir Tristram, illustrazione da Sir ThOMas Malory, The Birth Life and Acts of King Arthur, London, Dent, 1893 (edizione moderna di ID., Le Morte d'Arthur, London, William Caxton, 1485). 
nal ripete con pazienza le vicende che, dopo il duello, hanno riportato Tristano nella sua vera terra, sotto il vecchio sole, che dovrebbe restituirgli la sanità. Tuttavia lo sforzo generoso e commovente di riaccendere in Tristano l'appartenenza si infrange contro il suo quieto scetticismo: non crede alla guarigione, e neppure al ritrovamento della vera patria: tale egli considera piuttosto lo spazio senza sole, già da lui promesso a Isotta, al quale lo ha strappato il risveglio; la notte cosmica indicibile, dove il sapere coincide con l'oblio. Ma al giorno Tristano è stato riportato da un richiamo nostalgico di Isotta, che appartiene ancora a quel mondo, al regno del sole.

Dobbiamo intendere che Isotta è ancora prigioniera dell'universo sociale o, per esprimersi più sbrigativamente e brutalmente, di Marke? Non pare che sia così: la sua appartenenza al giorno sembra piuttosto data dal solo fatto di essere ancora viva; vale a dire, di nuovo e più che mai d'ora in avanti, la notte si sovrappone alla morte, e il desiderio amoroso si spinge nella vita come in una regione ostile, che prende ad ostaggi l'amore e l'amata. In ciò si realizza l'ansia del giorno che divora Tristano, e che non ha più nulla a che fare col sistema degli obblighi feudali: quel giorno è effettivamente spuntato per l'ultima volta con l'irruzione di Marke nella notte amorosa. Ma altrettanto è respinta da Tristano una nuova immagine del giorno, che ha forzato adesso le porte della notte e lo ha riportato, con un impeto selvaggio, dentro l'inganno della luce. Anche senza i contenuti mondano-sociali, ma per il solo fatto di essere identificata con la vita, essa si oppone all'amore, ed è equiparata, nel ricordo dell'eroe, alla lampada che Isotta tardava a spegnere.

Come allora, Tristano aspetta con impazienza che essa sia spenta, e che si faccia finalmente buio nella casa nuziale sua e di Isotta. Ricompare dunque l'equivalenza simbolica tra lo spegnimento della lampada e quello della vita, ma non più come il prezzo che Isotta era disposta a pagare, nell'asprezza della lotta mondana, per avere Tristano, bensì come desiderio autonomo, coerente e univoco.

La commovente purezza di Kurwenal fraintende diametralmente l'appello di Tristano alla sua amata: mentre egli torna a chiederle la morte, come nel terribile momento dell'approdo in Cornovaglia, il servo fedele insiste nell'aspettarla come sola chance del risanamento. Chi ha curato la ferita di Morold, un nemico gigantesco e implacabile, saprà bene curare il colpo portato da un traditore meschino. Per questo l'ha mandata a chiamare, e Tristano vivrà, purché ella stessa sia ancora viva - cosa che Tristano conferma prolungando l'equivoco: il rovello che l'ha destato è proprio il sussistere di Isotta in vita, o meglio, dovremmo dire, nei limiti e nella prigionia nella vita.

Ma se anche sono opposte le finalità per cui Tristano e Kurwenal aspettano Isotta, la notizia del suo arrivo esplode dentro Tristano nelle forme della gratitudine per Kurwenal. Alla sua fedeltà tocca un riconoscimento che fa da commosso congedo all'intero mondo cavalleresco: essa ha orientato il suo agire sempre, senza riserve, per Marke e contro Marke, contro Isotta e per Isotta, e sempre nella abnegazione di sé. È la più nobile e integra fra tutte le manifestazioni del giorno.

In nome della fedeltà e ingenuità di Kurwenal, così strettamente connesse che Kurwenal vive per Tristano senza mai capirlo, l'eroe gli chiede di andare di vedetta e dedi- 
care tutta la sua attesa all'arrivo di Isotta, che si presenta impetuosamente alla sua immaginazione. Kurwenal non vorrebbe lasciarlo solo; una notizia ancora negativa arriva dalla triste nenia del pastore, che già prima aveva richiamato Tristano alla veglia.

Tristano vi riconosce lo stesso suono delle notizie angosciose che ricevette nella sua infanzia, la morte del padre e la morte della madre. Essa è dunque la melodia che esprime il suo destino: quello di nutrire un desiderio infinito e inappagabile in vita, che si traduce in una inappagata volontà di morte. Due volte Isotta è stata vicinissima a dargliela: la prima volta quando, dopo averlo guarito dalla ferita, riconobbe in lui l'uccisore del suo fidanzato, la seconda quando gli offrì quello che entrambi credevano il veleno della riconciliazione.

Ma la speranza di una definitiva guarigione - non da una ferita, ma dalla vita - fu vanificata dal filtro di Brangania, che prima Tristano aveva difeso contro Isotta, ma che adesso è lui invece a maledire per la sofferenza immensa che gli ha procurato.

Il fatto è che, rispetto alla situazione precedente, al filtro è qui assegnato un diverso ruolo, in riferimento a un diverso conflitto. La possibilità di una esistenza notturna contrapposta all'esistenza sociale, che allora il filtro schiudeva, impedendo la morte, adesso è eliminata dal riconoscimento della morte come unico spazio amoroso - che è il grande evento compiutosi nella notte d'amore.

Conseguentemente, il filtro che impedisce la morte è considerato a posteriori, senza troppi riguardi per la farmacia di Brangania, nemico a sua volta dell'amore. Tanto è vero che assume i caratteri del bruciore, della luce, del soffocamento, i caratteri cioè del giorno che ogni volta rinasce per dare in pasto al sole, come a un uccello rapace, la sofferenza interminabile. Inoltre, mentre un tempo il filtro ha creato l'unione degli amanti, abolendo la distanza creata dai doveri sociali di Tristano, adesso è accusato di avere creato un desiderio solitario e unilaterale, che Isotta è chiamata non già ad appagare, ma a spegnere nella quiete suprema e definitiva.

Del resto la composizione del filtro come la definisce Tristano ( «io stesso l'ho preparato con l'angoscia di mio padre, con la sofferenza di mia madre, con le lacrime d'amore di ogni tempo, col riso e col pianto, con le gioie e con le ferite») richiama ben più la varietà contraddittoria e la vicenda dell'esistere che non l'immutabile valore della Minne, alla quale invece Isotta lo attribuiva.

È dunque forse ancora e solo l'ingenuità di Kurwenal a leggere quella di Tristano come una maledizione dell'amore, quando invece è più plausibile che sia soltanto una maledizione della vita, rivolta cioè a «quello che ci disturba, che impedisce a Tristano di amare Isotta per sempre, di vivere eternamente per lei».

Svenuto al culmine della sua invettiva, Tristano rinviene invocando l'arrivo della nave di Isotta, prefigurando la sua dolce immagine apportatrice di pace e conforto, e del perdono che un tempo gli aveva offerto. "Come sei bella!», dice, e anche la visione di lei che trascorre le onde fiorite sembra ricreare la condizione primaria dell'innamoramento.

E finalmente la certezza interna di Tristano (Isotta, che è tutto il suo mondo, non può essere scomparsa dal mondo) contagia la realtà: appena ha invitato Kurwenal a ve- 
dere ciò che lui vede così chiaro e luminoso, risuona di nuovo la zampogna, ma in un tono eloquente di letizia. Allora Kurwenal sale al posto di vedetta e scorge la nave: immagine di forza, di bellezza, di gioia.

E gioia è anche in Tristano per questa visione che pure occupa la luce del giorno; la si misura dalla sua preoccupazione e angoscia, quando la nave, compiendo il suo percorso regolare, scompare dietro uno scoglio. Teme la risacca, l'inesperienza o la malafede del pilota, che potrebbe essere amico di Melot; si sente perduto nei brevi attimi che solo per la sua impazienza costituiscono un ingiustificato ritardo della nave nel riapparire; insulta Kurwenal, poi si unisce al suo grido di gioia quando la nave riappare, ed esprime la sua gratitudine per lui destinandogli tutti i suoi averi (Kurwenal non avverte l'immediatezza sinistra di questa promessa). Già si distingue a bordo Isotta, che salta subito a terra dopo l'approdo. Su pressante richiesta di Tristano, Kurwenal si precipita ad aiutarla, dopo avere raccomandato a Tristano di rimanere nel suo letto.

Ma una volta solo, Tristano è preso dalla più grande agitazione; il desiderio che brucia in lui prospetta un'immagine ancora diversa del giorno: una radiosa voluttà assolata, una gioia delirante e febbrile, che si ribella e si svincola dalla morte. O almeno dall'attendere passivamente la morte come un dono dalle mani di Isotta. Tornato l'eroe di un tempo, Tristano vuole conquistarla, e conquistare con essa Isotta, che solo così potrà essere eternamente sua.

A entrambe va incontro, non solo trascurando il consiglio prudente di Kurwenal, ma strappandosi le bende e versando il proprio sangue, come già contro Morold: marca lucente dell'offerta eroica, ma segno anche di una trionfale fretta di far sparire il mondo.

Si sente in lontananza la voce carezzevole di Isotta, che giunge a Tristano come il segno carnale della vita, e dunque come la lampada che tarda a spegnersi. Andare verso di lei con l'ultimo impeto amoroso, significa finalmente spegnere la lampada e conquistare il buio amato. Pronunciando in risposta all'appello di Isotta il nome di lei, Tristano muore.

Sul suo corpo, Isotta pronuncia un disperato lamento, che è insieme un rimprovero. Morendo da solo, Tristano l'ha ancora una volta tradita: come quando si disponeva a bere da solo il veleno, e prima ancora, come quando ha separato i loro destini, obbligandola a sposare re Marke. Così a Isotta è stata sottratta la parte più preziosa della propria esistenza, l'ultima ora che ha sognato nell'angoscia dei giorni separati, la sola, eternamente breve, ultima felicità del mondo. Poi sarebbero morti insieme, secondo la promessa celebrata nella loro notte, e che aveva sedato gli ultimi dubbi dettati dalla volontà di vivere - quella stessa che ora, nel desiderio frustrato dell'ultima ora, più che mai pulsa ribelle.

Non da solo doveva morire Tristano e non della ferita che lei avrebbe potuto sanare, se non fosse arrivata troppo tardi. E tuttavia, in questa sensazione di essere beffata da un destino ironicamente sordo e squallido, Isotta legge meno precisamente che non nel suo rimprovero a Tristano. Non sarebbe infatti arrivata troppo tardi, se Tristano non avesse con feroce passione anticipato la propria fine; e dunque non è vero che Tri- 
stano sia morto della ferita, ucciso da un maligno agente estraneo. Anche a non voler ricordare che quella ferita è il prodotto preciso di una sua scelta, che ha armato la mano di Melot sottraendosi contemporaneamente alla difesa, Tristano se n'è una seconda volta appropriato, usandola come strumento di una precipitosa devozione alla notte e alla morte: questa è dunque la causa del suo ultimo tradimento, mentre i precedenti discendevano al contrario dalla sua servitù nei confronti del giorno.

Invocando ripetutamente, con meravigliosa infantile testardaggine, il risveglio di Tristano, Isotta si abbatte svenuta sul suo cadavere, quando rumori confusi annunciano l'inaspettata irruzione del mondo fin qui escluso.

Il pastore comunica a Kurwenal l'approdo di un'altra nave; su essa Kurwenal riconosce Marke e Melot - il re che ispirava le più alte perifrasi del linguaggio cortese è adesso nominato con sbrigativa brutalità, equiparato al detestatissimo nemico.

Anche nel linguaggio, Kurwenal rende testimonianza di un'idea univoca del mondo, incentrata sull'eroe appena morto. Poi, contro il presunto attacco, si dispone alla difesa con ordinata disperazione. Sbarra la porta e resta sconcertato di fronte alla opposta richiesta di Brangania, che con grande sorpresa vede fra i 'nemici' e accusa di tradimento. Quando l'ordine di sgombrare gli viene da Melot, Kurwenal assapora con gioia feroce la possibilità di vendicare il tradimento subito dal suo signore. Infatti Melot è abbattuto da lui, e muore col nome di Tristano sulle labbra.

Ancora Brangania cerca Isotta per darle notizie a suo dire buone, di fortuna, di salvezza, e inutilmente tenta di fermare Kurwenal, che si scaglia contro Marke e il suo seguito, dopo aver rivolto al re poche parole dignitose: "Qui infuria la morte. Qui non c'è altro da prendere, re. Se la vuoi, vieni!». Viene colpito e cade ai piedi del cadavere di Tristano, dopo averlo indicato all'ansiosa richiesta del re. Morendo si scusa con il suo signore di raggiungerlo nella morte che, a differenza di lui, non aveva desiderato lui che era quasi impazzito di felicità a veder tornare sulle labbra di Tristano la dolcezza della vita.

Come Isotta, anche Marke chiede disperatamente a Tristano di svegliarsi, e come Isotta si sente tradito dalla sua morte, che gli impedisce di dargli la più alta prova del suo affetto.

Le sue parole vengono subito dopo spiegate da Brangania, che ha preso Isotta tra le sue braccia, cercando di farla rinvenire: è stata lei a confessare al re la propria responsabilità nello scambio dei filtri. "Come fui felice, dice Marke, di trovare l'amico libero da ogni colpa!». Né il buon re si è limitato ad assolvere Tristano: riconoscendo che il filtro, che lo aveva portato a rompere il suo patto di fedeltà, portava alla luce una valore più profondo e più autentico, Marke ha rinunciato ai suoi diritti sulla sposa, e le è corso dietro per celebrare la propria rinunzia e l'unione di lei con Tristano.

$\mathrm{Ma}$ anche lui è arrivato troppo tardi, in tempo soltanto per accrescere il raccolto della morte. Chi porta la pace, non può gareggiare in velocità con l'impeto della sventura.

Forse però la generosa utopia di Marke incontra un ostacolo più specifico della generale vulnerabilità dell'uomo: anche nel suo caso essere arrivato troppo tardi è solo un modo di esprimere l'inattingibilità di chi è oggetto del beneficio; non è facile immagi- 
nare sposato a Isotta quel Tristano che la aspettava - e poi neppure l'ha aspettata - per morire; e che già nel loro primo incontro si chiedeva, con piena e candida certezza: «nella chiara luce del giorno, come poteva Isotta essere mia?».

La benignità come l'ostilità del mondo esterno sono ugualmente marginali, se non irrilevanti, alla storia d'amore.

Isotta infatti avverte appena l'affetto che la circonda, e solo per coinvolgere i presenti nella sua ultima invocazione e contemplazione di Tristano.

Come nella notte d'amore, a un'affermazione tenace e dolente della volontà di vita tiene dietro da parte sua un'appropriazione sovrana della morte come essenza dell'anima amante. Qui non c'è di mezzo un ragionamento persuasivo, ma il risveglio dallo svenimento che la porta a guardare il corpo dell'amato con altri occhi, aspettando da lui non la resurrezione individuale ma il fluire intoccabile della vita cosmica: «Dolce e lieve, come sorride, come apre graziosamente gli occhi, lo vedete, amici? Non lo vedete? Come il cuore fieramente gli si gonfia e pieno e nobile sgorga nel petto! Come dalle labbra gioiosamente dolci fluisce un dolce respiro! Amici, vedete: non sentite, non vedete? Odo io soltanto questa melodia, che così lieve e meravigliosa, piangendo nella gioia, esprimendo tutto, dolce e conciliante, risuona da lui e penetra in me, si libra in alto, echeggia soavemente e mi avvolge nel suono? I suoni limpidi che mi circondano sono forse onde di morbide brezze? O sono vortici di piacevoli vapori? Come si gonfiano e mormorano intorno a me! Devo respirarli, ascoltarli? Devo berli, immergermi in loro? Dolcemente esalare nei vapori? Nel mare ondeggiante, nel tutto palpitante del respiro del mondo, naufragare, affondare inconsciamente, piacere supremo!». 


\section{TRISTAN UND ISOLDE}

Libretto di Richard Wagner

Edizione a cura di Riccardo Pecci, con guida musicale all'opera 


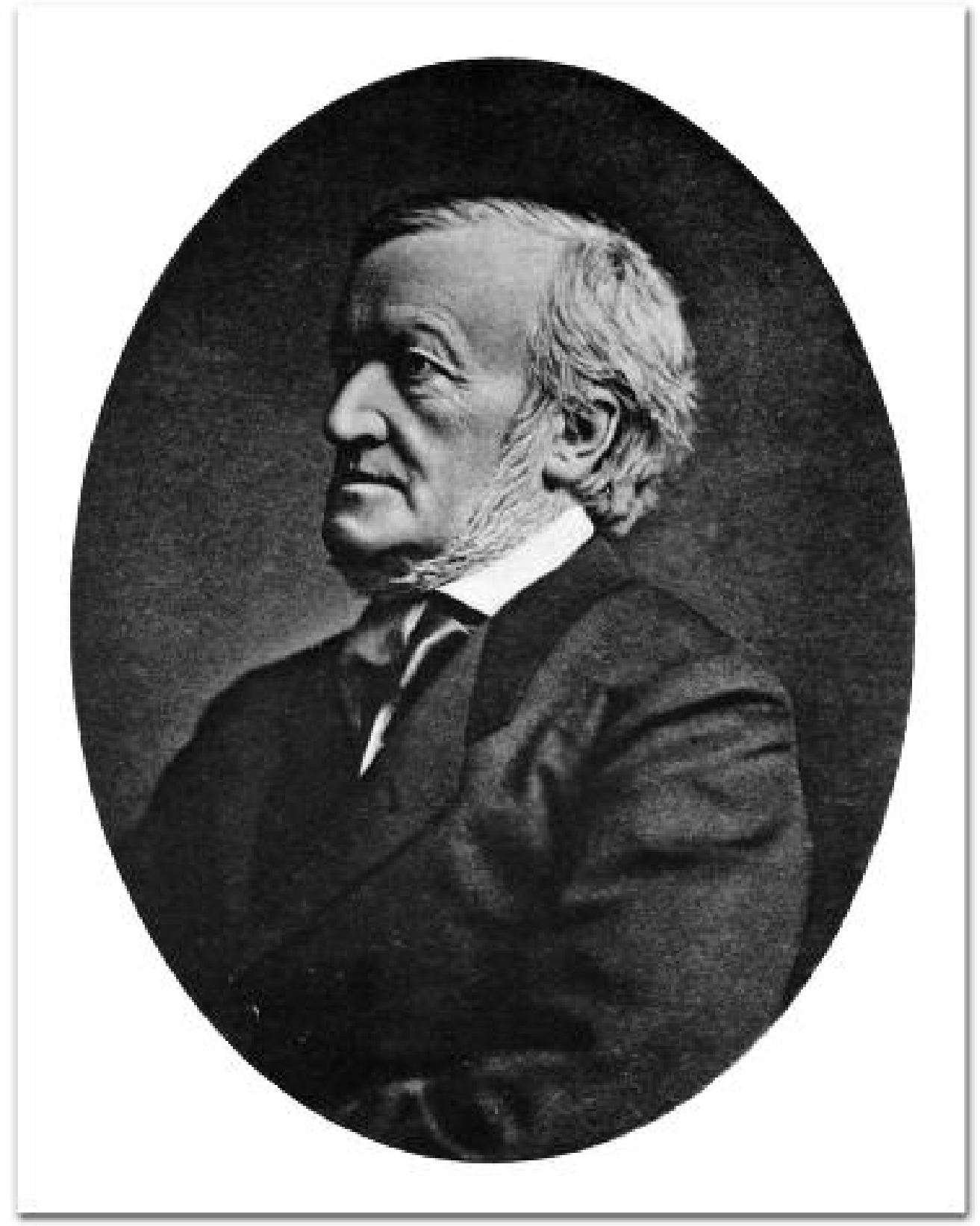

Richard Wagner in una foto di Joseph Albert (Monaco, 1 maggio 1882). 


\title{
Tristan und Isolde, libretto e guida all'opera
}

\author{
a cura di Riccardo Pecci
}

Tristan und Isolde, e basta: non dramma, non «azione» (Handlung) in tre atti. È con la lapidaria nudità di tre parole, senza sottotitoli di sorta, che Wagner apre il libretto del Tristan nella veste da lui stesso curata per le sue Gesammelte Schriften und Dichtungen: ${ }^{1}$ un'anomala nudità che ben si presta ad enfatizzare il ruolo del tutto speciale che Tristan ricopre nella storia del teatro musicale europeo. In linea con recenti operazioni editoriali, ${ }^{2}$ si è deciso appunto di utilizzare come base dell'edizione del libretto del Tristan und Isolde il testo delle Gesammelte Schriften. ${ }^{3}$ È infatti certamente questa (per assetto metrico, scelte ortografiche, punteggiatura) la forma attentamente meditata nella quale Wagner avrebbe voluto che il suo poema si presentasse agli occhi dei suoi lettori: forma preferibile, per attenzione ai valori poetico-letterari, a quella che viene normalmente offerta al pubblico. ${ }^{4}$ Ad essa sono state nondimeno apportate tutte le modifiche strettamente necessarie per riallinearlo al testo effettivamente intonato nella partitura, cui si attiene a sua volta la storica traduzione italiana di Guido Manacorda ${ }^{5}$ (autore negli anni Venti e Trenta del secolo scorso di versioni commentate dei poemi wagneriani per la Biblioteca Sansoniana Straniera «che per precisione, qualità letteraria e vastità dell'apparato esegetico hanno pochi termini di confronto anche fuori del nostro paese»). ${ }^{6}$ I punti di divergenza della partitura rispetto alla lezione delle Gesam1873.

${ }_{1}^{1}$ Richard WaGner, Gesammelte Schriften und Dichtungen, 10 voll., Leipzig , E. W. Fritzsch, 1871-1883, vII,

2 Vedi Richard Wagner, Tristan und Isolde. Textbuch mit Varianten der Partitur, a cura di Egon Voss, Stuttgart, Reclam, 2003, e anche Wagner's «Ring of the Nibelung»: A Companion. The full German text with the acclaimed English translation by Stewart Spencer and commentaries by Barry Millington, Elisabeth Magee, Roger Hollinrake and Warren Darcy, a cura di Stewart Spencer e Barry Millington, London, Thames \& Hudson, 1993. Questa edizione del poema del Ring des Nibelungen pubblica il testo delle Gesammelte Schriften, rettificandone didascalie e testo sulla base delle varianti della partitura - tra l'altro, ben più sostanziose che non nel caso del Tristan. (Con una scelta infelice, tuttavia, le didascalie sono offerte solo in traduzione inglese, senza possibilità di riscontro a fronte.)

3 Secondo l'edizione stabilita da Egon Voss (cfr. nota precedente). Si è tenuto conto anche del testo del poema in Richard Wagner, Werke, Schriften und Briefe, a cura di Sven Friedrich, Berlin, Direct Media, 2004, pp. 3070-3188.

${ }^{4}$ Un esempio classico di edizione in commercio del libretto del Tristan è ID., "Tristan und Isolde»: Textbuch, Einführung und Kommentar, a cura di Kurt Pahlen e Rosmarie König, Mainz, Schott, @ 1998, 2010 (19801). $1932^{2}$

${ }_{5}$ ID., Tristano e Isolda, a cura di Guido Manacorda, Firenze, Le Lettere, 1994 (ed. or. Firenze, Sansoni,

6 MAurizIo GIANI, Luigi Torchi traduttore di Wagner, in Tra le note. Studi di lessicologia musicale, a cura di Fiamma Nicolodi e Paolo Trovato, Fiesole, Cadmo, 1996, pp. 93-104: 93. 
melte Schriften (coll'esclusione delle varianti meramente grafiche o interpuntive) sono segnalati nella colonna del testo tedesco dal grassetto grigio e da esponenti in cifre romane, che rinviano a note a piè di pagina. ${ }^{7}$ E stato effettuato qualche piccolo cambiamento alla versione di Manacorda per uniformarla al testo tedesco della nostra edizione. ${ }^{8}$

Le cifre arabe in esponente nella sezione tedesca del libretto rimandano alla guida all'ascolto che scorre a piè di pagina. L'analisi musicale è stata condotta sull'edizione critica della partitura approntata da Isolde Vetter ed Egon Voss per i tipi della Schott, nell'ambito dei Sämtliche Werke di Wagner (1970-). ${ }^{9}$ Gli esempi musicali sono riduzioni in suoni reali della partitura o (più spesso) di un sottoinsieme significativo delle parti orchestrali annotate dal compositore.

\begin{tabular}{|c|c|}
\hline ATTO PRIMO & \\
\hline ATTO SECONDO & \\
\hline ATTO TERZO & \\
\hline APPENDICI: & $\begin{array}{l}\text { L'orchestra } \\
\text { Le voci }\end{array}$ \\
\hline
\end{tabular}

7 Parole, versi e didascalie in grassetto grigio senza alcun esponente di nota devono essere intesi come aggiunte della partitura al testo delle Gesammelte Schriften (sigla GS); nei casi invece in cui sono seguiti da un esponente in cifre romane, esso rinvia a una nota a piè di pagina dove si riporta la lezione delle Gesammelte Schriften. Sono state segnalate anche alcune divergenze grafiche o di interpunzione che incidono sul significato del verso.

${ }^{8}$ Tali cambiamenti sono segnalati dal grassetto grigio. Per rispetto dell'integrità dei versi di Manacorda si sono conservati i nomi dei personaggi da lui scelti - peraltro o di uso comune (Tristano, Brangania) o di suggestivo «sapore» wagneriano, per usare un termine dello stesso Manacorda (Isolda, in luogo del più corrente Isotta). Abbiamo fatto un'unica eccezione per "Marco», che non ci è parso adeguatamente giustificato ed è stato sostituito in linea con le traduzioni più recenti - con il nome tedesco (Marke). Qualche intervento sulla punteggiatura è stato effettuato tacitamente. Abbiamo conservato le occasionali parentesi quadre di Manacorda, con le quali il traduttore indicava alcune integrazioni atte a rendere la sua versione più comprensibile.

9 RICHARD WAGNER, Tristan und Isolde. Handlung in 3 Aufzügen WWV 90, a cura di Isolde Vetter ed Egon Voss, Mainz, Schott, 1990-1993 (Sämtliche Werke, Reihe A: Notenbände mit Kritischem Bericht im Anhang, Band 8/I-III). Cfr. la ristampa negli Eulenburg Miniature Scores: ID., Tristan und Isolde, London-Mainz-Madrid, Eulenburg, 2001. Abbiamo consultato anche l'edizione Peters della partitura (circa 1911: rist. New York, Dover, 1973) e della riduzione per canto e pianoforte (di Felix Mottl e Gustav F. Kogel: EP Nr. 3407, @ 1942); entrambe recano le annotazioni scenico-musicali di Mottl (1856-1911), direttore nel 1886 della prima esecuzione a Bayreuth del Tristan. I luoghi citati sono individuati mediante l'atto e il numero di battute; le note con iniziale maiuscola identificano tonalità maggiori ( $\mathrm{Sib}=\mathrm{Si}$ bemolle maggiore), minuscola per le minori. 


\section{Richard Wagner \\ TRISTAN \\ UND ISOLDE}

PERSONEN

TRISTAN

KÖNIG MARKE

ISOLDE

KURWENAL

MELOT

BRANGÄNE

EIN HIRT

EIN STEUERMANN

EIN JUNGER SEEMANN

Männerchor: Schiffsvolk, Ritter und Knappen
Tenor $^{\mathrm{I}}$

Baß

Sopran

Bariton

Tenor

Sopran

Tenor

Bariton

Tenor

1. und 2. Tenor, 1. und 2. Bass

\section{Schauplatz der Handlung}

Erster Aufzug: Zur See auf dem Verdeck vor Tristans Schiff, während der Überfahrt vor Irland nach Kornwall.

Zweiter Aufzug: In der Königlichen Burg Markes in Kornwall.

Dritter Aufzug: Tristan Burg in Bretagne.

\footnotetext{
${ }^{\mathrm{I}}$ Il testo segnalato in grassetto grigio nel frontespizio non proviene da GS bensì dalla prima edizione della partitura (Leipzig, Breitkopf \& Härtel, 1860).
} 


\section{Richard Wagner \\ TRISTANO E ISOLDA}

PERSONAGGI

TRISTANO

RE MARKE

ISOLDA

KURWENAL

MELOT

BRANGANIA

UN PASTORE

UN PILOTA

UN GIOVANE MARINAIO
Tenore

Basso

Soprano

Baritono

Tenore

Soprano

Tenore

Baritono

Tenore

Coro di voci maschili: equipaggio, cavalieri e scudieri Tenori I e II, Bassi I e II

LUOGHI DELL'AZIONE

Atto primo: In mare, sul ponte della nave di Tristano, durante la traversata dall'Irlanda alla Cornovaglia.

Atto secondo: Nel castello reale di Marke in Cornovaglia.

Atto terzo: Castello di Tristano in Bretagna. 


\section{«Fra le spire d'un grande serpente»: il preludio a Tristan und Isolde}

Il 'serpente' che inquietava Puccini (si veda il racconto di Guido Marotti, qui a p. 9), ha sì la testa protesa in avanti, ma anche una coda saldamente ancorata al passato. La modernità di Tristan und Isolde, al tempo stesso, affonda radici profonde (spesso misconosciute) nella tradizione. Guardiamo dunque un poco dentro questo preludio - o meglio, Einleitung, «introduzione», come lo definisce la partitura. Nelle parole di Wagner, il tema di questa introduzione orchestrale è il «desiderio [Sehnsucht]»: un desiderio «inestinguibile, una brama [Verlangen] che si rigenera eternamente». Al punto che è possibile un' «unica liberazione [Erlösung]: morire, perire, soccombere [Untergehen], non svegliarsi più [Nichtmehrerwachen]!». ${ }^{1}$

Questa intenzione di fondo ispira a Wagner «un passaggio molto sviluppato e articolato" che copre circa dieci minuti di musica: nel corso del preludio, "la brama insaziabile [das unersättliche Verlangen]» viene mostrata dall'orchestra nella sua evoluzione

dalla confessione più rattenuta, dall'attrazione più tenera e passando attraverso sospiri ansiosi, speranze e sgomenti, lamenti e desideri, piacere e tormento, fino a toccare l'impeto più travolgente, lo sforzo violento di trovare una breccia [...]. Invano! Impotente il cuore torna a struggersi di desiderio, un desiderio senza meta perché ogni meta è solo un nuovo desiderio. Finché, nell'estremo languore, allo sguardo ormai velato si affaccia il presentimento di una voluttà estrema: è la volontà della morte, del non-essere-più [des Nichtmehrseins].

In queste poche righe, come vedremo, è impresso con ragguardevole precisione l'itinerario musicale del Vorspiel. S'inizia appunto dalla "confessione più rattenuta»: i violoncelli intonano pianissimo il motivo del Dolore (Leidensmotiv: $x$ nell'es. 1), ${ }^{2}$ una linea melodica che si flette verso il basso con passo semitonale, alla quale repli-

${ }^{1}$ Richard WAGner, Programmatische Erläuterungen. I. «Tristan und Isolde»: Vorspiel, in ID., Werke, Schriften und Briefe, a cura di Sven Friedrich, Berlin, Direct Media, 2004, pp. 6626-28. La traduzione (modificata) è quella di ID., Scritti scelti, a cura di Dietrich Mack, introduzione di Ernst Bloch, Parma, Guanda, 1988, pp. 140-141.

2 I nomi che attribuiamo per comodità ai Leitmotive della partitura sono quelli suggeriti da ALFRED LORENZ, Das Geheimnis der Form bei Richard Wagner, 4 voll., II: Der musikalische Aufbau von Richard Wagners "Tristan und Isolde», Berlin, Max Hesse, 1926 (rist. Tutzing, Schneider, 1966). A sua volta, Lorenz segue largamente (ma non pedissequamente) le classiche proposte di HaNs vON WolZOGEN, Thematischer Leitfaden durch die Musik zu Richard Wagner's «Tristan und Isolde», nebst einem Vorworte über den Sagenstoff des Wagner'schen Dramas, Leipzig, Feodor Reinboth, $1888^{3}$; integrandole con qualche spunto di ERNST KURTH, Romantische Harmonik und ibre Krise in Wagners "Tristan», Berlin, Max Hesses Verlag, $1923^{3}$. 
ca specularmente l'oboe con un motivo cromatico ascendente, quello del Desiderio (Sehnsuchtsmotiv: $y$ nell'es. 1). Nel loro punto d'incontro (b. 2), dai legni si sprigiona la «chiave magica» di cui parlava Puccini: l'enigmatico accordo dissonante $\mathrm{Fa}_{2}-\mathrm{Si}_{2}$ Re\#- $\#_{3}$ Sol\# $(z$ nell'es. 1), ovvero l'“accordo del Tristan' (Tristanakkord) per antonomasia. ${ }^{3}$ Nella battuta successiva, il Tristanakkord risolve sulla settima di dominante di la, tonalità cui il preludio allude nei suoi snodi strutturali, senza però mai acquietarsi in essa.

ESEMPIO 1 (Einleitung, bb. 1-3)

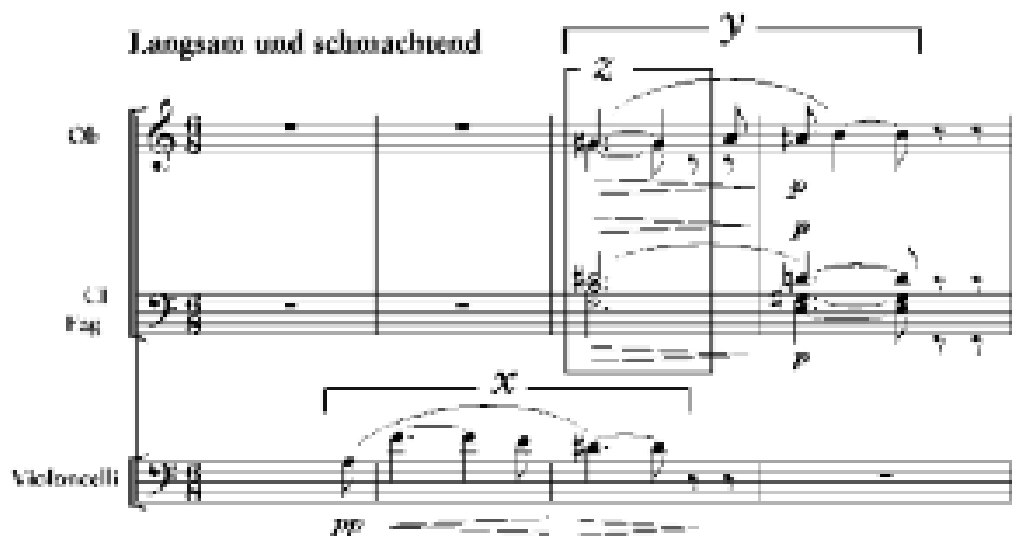

Il procedimento si rivelerà assolutamente caratteristico dello stile del Tristan: la tensione del primo accordo (es. 1z) risolve infatti su un secondo accordo (la settima di dominante) che tuttavia è a sua volta dissonante, e dunque bisognoso di risoluzione. E così via, potenzialmente all'infinito: perfetta traduzione musicale di quel desiderio che è al centro del Tristan und Isolde, «un desiderio senza meta perché ogni meta è solo un nuovo desiderio».

Il contenuto di queste tre battute (motivi $x, y$, accordo $z$ ) determinerà in modo decisivo il suono del Tristan. In particolare, già Hans von Wolzogen individuava nel motivo del Desiderio l'Hauptmotiv, il «motivo principale» dell'intero dramma e dell'intera musica del Tristan: ${ }^{4}$ di fatto, questa cellula di quattro note a distanza di semitono $\left(\mathrm{Sol} \#^{3}-\mathrm{La}^{3}-\mathrm{La} \#^{3}-\mathrm{Si}^{3}\right)$ sarà virtualmente implicata in ogni pagina dal cromatismo pervasivo della scrittura di Wagner. ${ }^{5}$

Su questo materiale, Wagner imbastisce ora un vero e proprio 'tema del Desiderio' (Sehnsuchtsthema), di diciassette battute, dalla struttura riconoscibile, che funzionerà

${ }^{3}$ Nell'efficace gergo musicale anglosassone, si tratta di un accordo di settima semi-diminuita (balf-diminished seventh chord), o 'accordo semi-diminuito', la cui struttura intervallare è omologa alla cosiddetta $7^{\mathrm{a}}$ di terza specie della nostra manualistica.

4 Wolzogen, Thematischer Leitfaden cit., p. 15.

5 ThOMAs S. Grey, Wagner's musical prose: texts and contexts, Cambridge, Cambridge University Press, 1995, p. 355. 
da primo tema dell'intero preludio: cominciando con la ripetizione in progressione dell'es. 1 (il punto d'arrivo è ora una terza minore sopra). ${ }^{6}$

La conclusione del tema è segnata da un'intensità crescente, e consiste in un' «ampia e peculiare anacrusi dominantica» ${ }^{7}$ che si scarica infine in un'enfatica cadenza d'inganno V-VI in la (es. 2). Ancora una volta, la meta della tonica non è stata pienamente raggiunta.

ESEMPIO 2 (bb. 16-23)
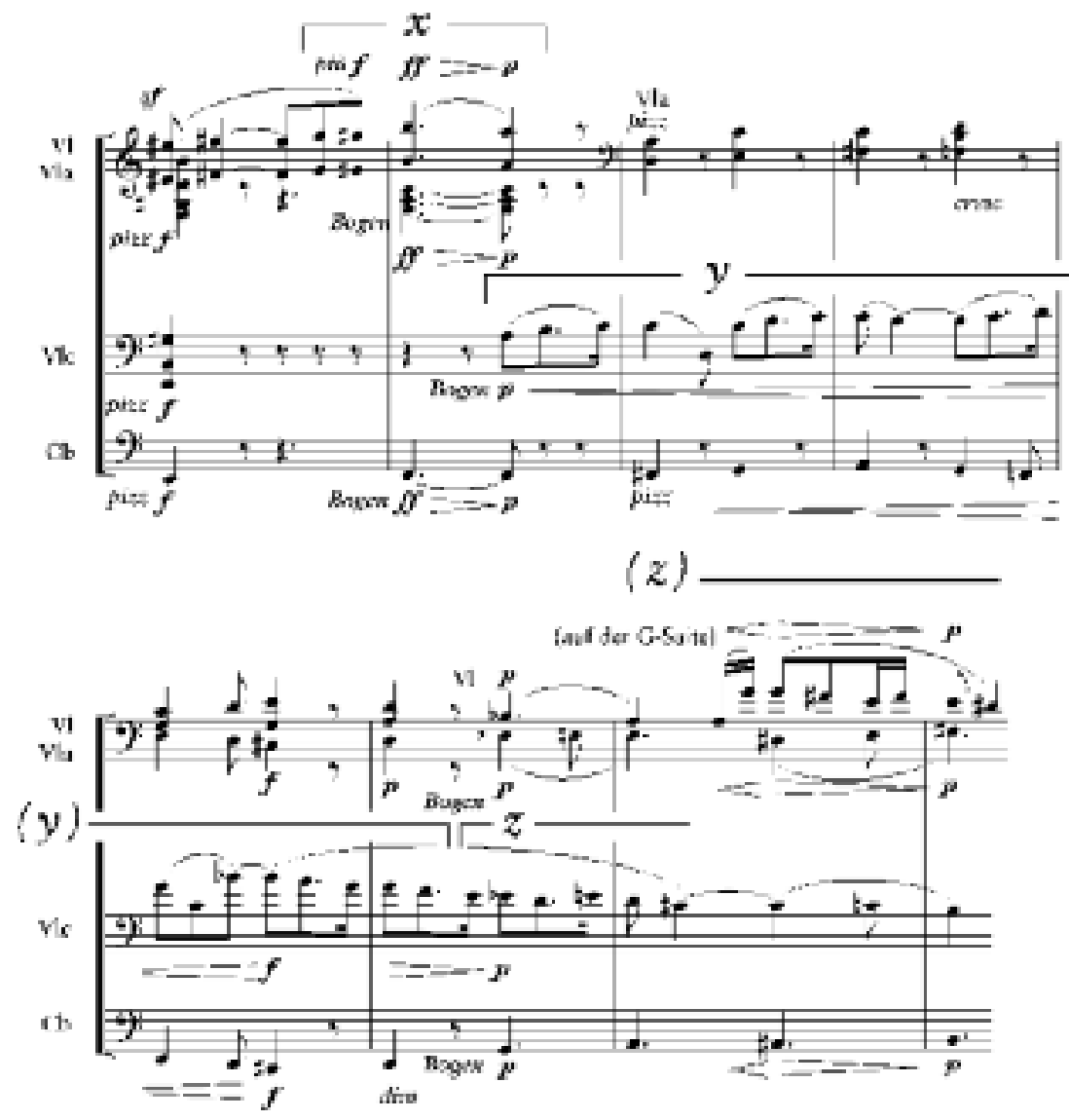

${ }^{6}$ Cfr. Alfred Lorenz, Die Formale Gestaltung des Vorspiels zu «Tristan und Isolde», «Zeitschrift für Musikwissenschaft», 5, 1922/23, pp. 546-557 (ripubblicato in Zur musikalischen Analyse, a cura di Gerhard Schuhmacher, Darmstadt, Wissenschaftliche Buchgesellschaft, 1974, pp. 455-474, poi confluito in ID., Das Geheimnis der Form, pp. 12-28). Una traduzione inglese è in RiCHARD WAGNER, Prelude and Transfiguration from "Tristan and Isolde», a cura di Robert Bailey, New York, Norton, 1985, pp. 204-20: il volume più ampio finora consacrato al nostro preludio, che ripubblica i principali studi analitici sull'argomento. Questa raccolta va ora integrata almeno con il capitolo VI di ERIC CHAFE, The Tragic and the Ecstatic: The Musical Revolution of Wagner's «Tristan und Isolde», Oxford, Oxford University Press, 2005, e Joseph Kerman, The Prelude and the play, in Richard Wagner: «Tristan und Isolde», a cura di Arthur Groos, Cambridge, Cambridge University Press, 2011, pp. 53-68.

${ }^{7}$ KURTH, Romantische Harmonik, p. 320. 
La cadenza d'inganno è resa incredibilmente espressiva da un'appoggiatura in ottava $\left(\mathrm{Si}_{3}-\mathrm{Si}_{4}\right)$ a distanza tritonale dal basso $\left(\mathrm{Fa}_{1}\right)$ : eseguita in fortissimo da violini e viole, essa fa parte del cosiddetto motivo del Fato (Verhängnismotiv: $x$ nell'es. 2). Il La, nota di risoluzione dell'appoggiatura, viene immediatamente preso in consegna dai violoncelli, che ne fanno il punto di partenza del secondo tema del preludio: una melodia larga e diatonica (vedi $y$ e $z$ nell'es. 2), che nell'atto primo si farà interamente carico del «segreto di un amore non confessato» 8 celato nello sguardo di Tristan a Isolde (di qui, il nome corrente di motivo dello Sguardo, o Blickmotiv). L'es. 2 salda dunque i due temi del preludio in un unico "complesso», ${ }^{9}$ destinandolo a un ruolo cruciale nell'economia musicale del Tristan - a cominciare dal preludio stesso.

La sezione centrale del preludio dà poi voce a quel gioco inconcludente di «sospiri ansiosi, speranze e sgomenti, lamenti e desideri, piacere e tormento» di cui scriveva Wagner: una sezione tutta costruita sui materiali fin qui esposti, nella quale udiamo per la prima volta il cupo profilo di un motivo nei bassi ( $x$ nell'es. 3 ).

ESEMPIO 3 (bb. 27-29)

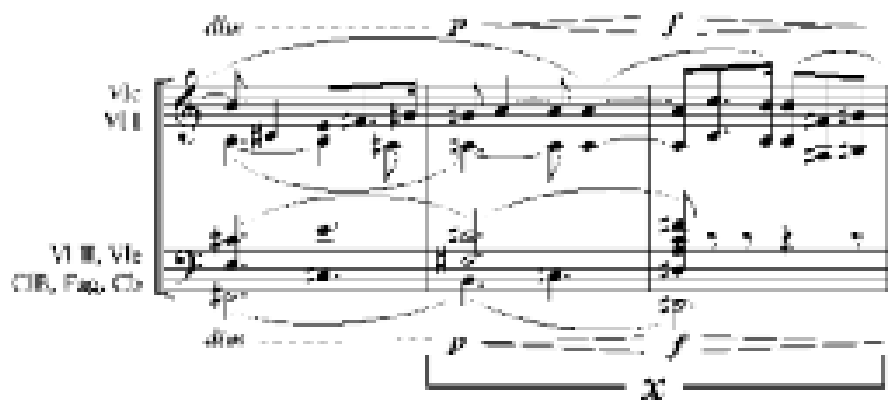

Incapace di saziarsi, il desiderio cresce nella musica verso «l'impeto più travolgente, lo sforzo violento di trovare una breccia», in uno slancio che si giova di una nuova figura plastica degli archi:

ESEMPIO 4 (bb. 63-64)

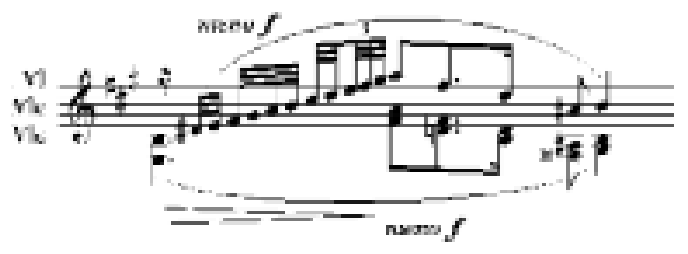

Ma l'es. 4 è appena la superficie della musica: la figura dissimula infatti una ripresa integrale del complesso formato dai due temi del Desiderio e dello Sguardo (da b. 66),

${ }^{8}$ ERnest Newman, Le opere di Wagner [The Wagner Operas, 1991], Milano, Mondadori, 1981, p. 219.

9 Kerman, The Prelude and the play cit., p. 56. 
che lungo un crescendo mozzafiato tentano effettivamente di aprirsi una breccia. Tale è lo sforzo che l'asse tonale ne viene provvisoriamente spostato verso il Mib, una tonica remotissima da quella originaria. «Invano!", scriveva laconico Wagner: e infatti, nella sezione finale del preludio (da b. 86), la musica sconfitta ripiega su se stessa e recupera i toni sommessi dell'apertura, in una ripresa frammentaria e dolente del complesso tematico Desiderio-Sguardo, che si lascia alle spalle il la e muove verso il do della prima scena dell'atto (rivelando il suo carattere di introduzione). Alla fine del preludio, non resta in orchestra che una volontà di morte, del «non-essere-più».

Della musica di questo preludio, lo scorcio iniziale si è ormai imposto come una sorta di 'firma' di Wagner: ed è regolarmente contrabbandato come manifesto di un'originalità irriducibile a qualunque schema. Eppure, come abbiamo visto, il 'serpente' sinfonico del Vorspiel si mostra in bilico tra passato e futuro. La sovversione wagneriana non rinuncia cioè a giocare ambiguamente con gli elementi forniti dalla tradizione: ad esempio, utilizzando lo schema Esposizione-Ripresa, e perfino il consueto bitematismo sonatistico (il secondo tema del preludio si permette addirittura di cominciare regolarmente nella tonalità relativa di la, Do). Anche per quanto riguarda l'avvio del preludio, la caccia ai possibili modelli ha individuato precedenti in Beethoven, Spohr, Liszt, Berlioz ecc. Senza bisogno di moltiplicare gli esempi e di uscire dal grande repertorio della musica europea, in fondo ascoltiamo qualcosa di non molto lontano dalle battute iniziali del Tristan già nell' insospettabile' Jupiter di Mozart (si provi a decelerare mentalmente un passaggio dal Molto Allegro dal Finale, di cui riproduciamo un frammento nell'es. 5).

ESEMPIO 5: Mozart, Sinfonia in Do maggiore KV 551 Jupiter, Iv, bb. 243-46

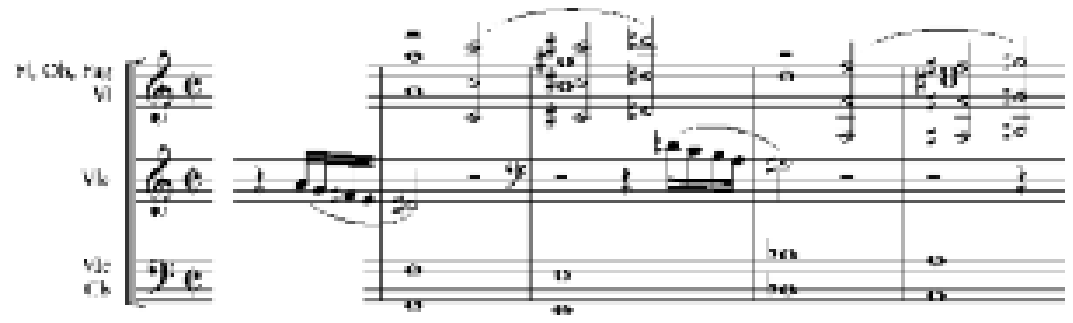




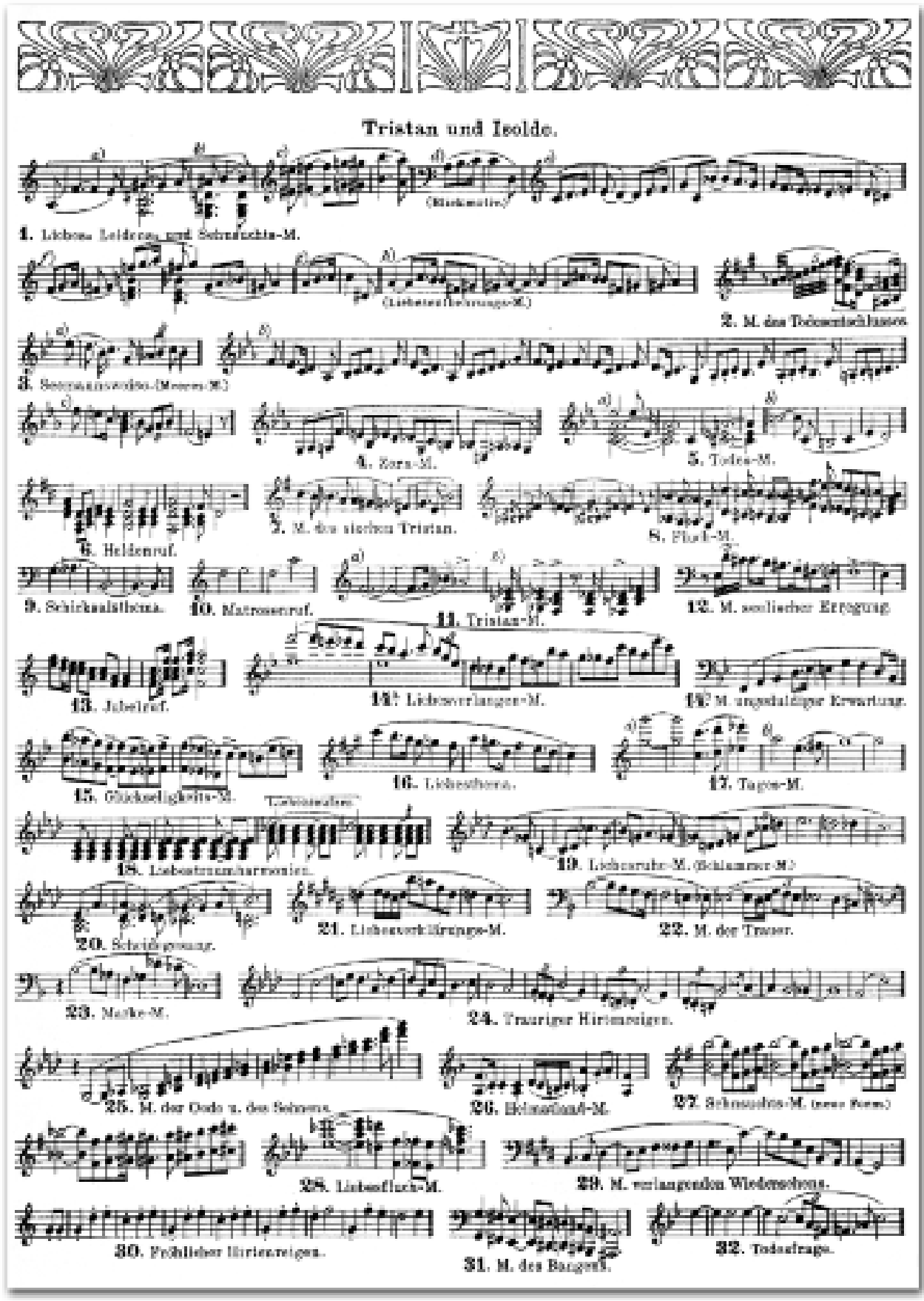

Tavola dei Leitmotive redatta da Carl Waack e riprodotta nello spartito di Tristan und Isolde stampato a Lipsia da Breitkopf \& Härtel, 1914. 


\section{ERSTER AUFZUG}

\section{ERSTE SZENE ${ }^{\mathrm{I}}$}

Zeltartiges Gemach auf dem Vorderdeck eines Seeschiffes, reich mit Teppichen behangen, beim Beginne nach dem Hintergrunde zu gänzlich geschlossen; zur Seite führt eine schmale Treppe in den Schiffsraum hinab.

(Isolde auf einem Rubebett, das Gesicht in die Kissen gedrückt. - Brangäne, einen Teppich zurückgeschlagen haltend, blickt zur Seite über Bord)

STIMME EINES JUNGEN SEEMANNES (aus der Höhe, wie vom Maste her, vernehmbar)

West-wärts ${ }^{1}$

schweift der Blick;

ost-wärts

streicht das Schiff.

Frisch weht der Wind

der Heimat zu: -

\section{ATTO PRIMO}

\section{SCENA PRIMA}

Camera a foggia di tenda, sulla tolda di un naviglio, riccamente drappeggiata con tappeti; da principio, completamente chiusa verso il fondo. Da un lato, una scaletta scende sottocoperta.

(Isolda su un divano, il viso stretto tra i cuscini. Brangania tenendo rialzato il lembo d'un tappeto, guarda di lato, sopra bordo)

VOCE DI UN GIOVANE MARINAIO (si sente come se venisse dall'alto dell'albero)

Verso occidente

erra lo sguardo;

verso oriente

scivola il naviglio.

Fresco soffia il vento

verso la patria:

\footnotetext{
${ }^{\text {I }}$ GS: la suddivisione in scene manca.
}

${ }^{1}$ I violoncelli soli avevano inaugurato la musica del preludio: ora tocca alla voce nuda di un marinaio l'intonazione di una composita melodia in do (che inizia con un'allusione discreta agli intervalli del motivo del Dolore, es. $1 x$ ). La stessa melodia vocale tornerà al principio della scena successiva, serrando tonalmente e motivicamente la prima scena di Tristan und Isolde. Scena emblematica del metodo di lavoro del Wagner maturo, alla quale non a caso è stata dedicata nel 1895 un'analisi pionieristica, quella di Jean Hubert (Étude sur quelques pages de Richard Wagner), che ci servirà a entrare nel linguaggio formale del Tristan. Qui Hubert mostra come Wagner realizzi un'unità di tipo sinfonico utilizzando un materiale tematico gerarchizzato, e sottoposto a procedimenti di frammentazione, ripetizione, progressione, imitazione e trasformazione dei motivi. Quattro battute energiche (kräftig) del canto del marinaio (es. 6) forniscono il «tema generatore» (thème générateur) della musica, che funzionerà da pivot (da cardine, da elemento centrale) per l'intera scena.

ESEMPIO 6 (I, bb. 116-119)

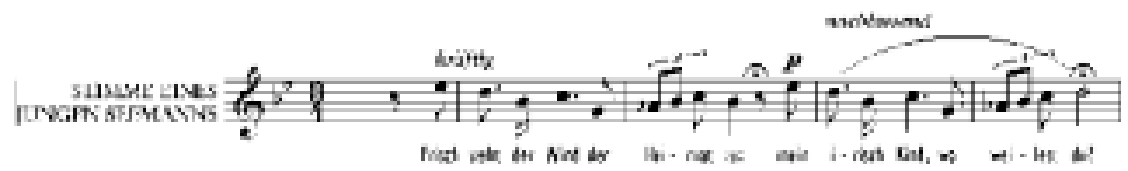

Ad esso Wagner affianca vari «temi sussidiari, complementari» (thèmes subsidiares): distinguibili a loro volta, secondo Hubert, in motivi che «sono già stati uditi e lo saranno ancora lungo l'opera» (e che dunque riguardano il dramma nella sua interezza) e motivi che invece posseggono un mero valore locale. Tra i primi, il motivo dello Sguardo (es. 2y), che in una versione lebhaft («vivace») scuote Isolde dalla sua immobilità iniziale. E quello del Desiderio (es. 1y), che viene qui peraltro intonato per la prima volta (alle parole «zu tobender Stürme I wüthendem Wirbel!»: «Di rombanti procelle I al furente vortice!») nell'esplosione selvaggia di Isolde ( Entartet Geschlecht»: "Razza degenere») che fa da centro della scena, incorniciata dagli interventi di Brangäne. Ma questo gruppo di motivi conta anche quello debuttante della Collera di Isolde (Zornmotiv: es. 7), che accompagna nervoso nel forte degli archi lo scatto della donna in apertura di scena («Wer wagt mich zu höhnen?»: «Chi osa schernirmi?»). 
mein irisch Kind,

wo weilest du?

Sind's deiner Seufzer Wehen, die mir die Segel blähen? -

Wehe! Wehe, du Wind!

Weh! Ach wehe, mein Kind!

Irische Maid,

du wilde, minnige Maid!

ISOLDE (jäh auffahrend)

Wer wagt mich zu höhnen?

(Sie blickt verstört um sich)

Brangäne, du? -

Sag, wo sind wir?

BRANGÄNE (an der Öffnung)

Blaue Streifen

stiegen im ${ }^{\text {II }}$ Westen auf;

sanft und schnell

segelt das Schiff;

auf ruhiger See vor Abend

erreichen wir sicher das Land.

ISOLDE

Welches Land?

BRANGÄNE

Kornwalls grünen Strand.

ISOLDE

Nimmermehr!

Nicht heut, noch ${ }^{\text {III }}$ morgen!

BRANGÄNE (läßt den Vorhang zufallen, und eilt bestürzt zu Isolde)

Was hör' ich? Herrin! Ha! mia fanciulla d'Irlanda,

dove te ne stai?

Sono gli aliti dei tuoi sospiri,

che mi gonfiano le vele?

Ve', ve', o vento!...

Ve', ah ve', mia bimba!...

o fanciulla d'Irlanda,

o selvaggia, amorosa fanciulla!

ISOLDA (improvvisamente sobbalzando)

Chi osa schernirmi?

(guarda insospettita intorno a sé)

Sei tu Brangania?

Dimmi... dove siamo?

BRANGANIA (all'apertura [della tenda])

Strie d'azzurro

si sono alzate ad oriente;

soave, veloce

veleggia il veliero:

su mare tranquillo avanti sera,

toccheremo sicuri la terra.

ISOLDA

Quale terra?

BRANGANIA

La verde spiaggia di Cornovaglia.

ISOLDA

Giammai!

Non oggi, né domani!

BRANGANIA (lascia cadere la tenda e s'affretta, sbalordita, ad Isolda)

Che odo io? Signora! Ah!

segue nota 1

ESEMPIO 7 (bb. 137-139)

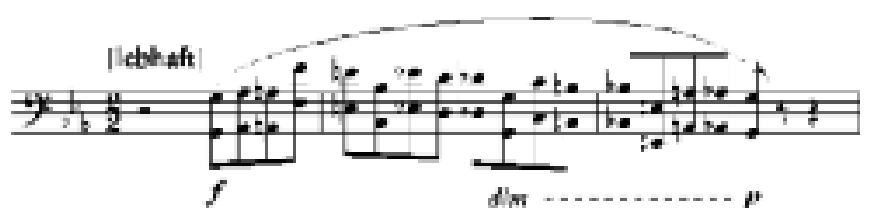

II GS: «in». Merita un'osservazione anche la parola Westen («occidente»), attestata sia dalle Gesammelte Schriften che dalla partitura. Il termine sembra infatti palesemente fuori posto: se si naviga dall'Irlanda verso la Cornovaglia, si procede «verso levante» (ost-wärts), come canta il giovane marinaio, dunque le «strisce azzurre» della terraferma non potranno sorgere a ponente! Ciò spiega perché nelle edizioni musicali e in quelle del libretto (compresa la fonte utilizzata da Manacorda) la parola venga spesso rettificata in Osten: «oriente», come traduce appunto Manacorda.

III GS: «nicht». 
ISOLDE (wild vor sich hin)

Entartet Geschlecht, unwert der Ahnen!

Wohin, Mutter, vergabst du die Macht,

über Meer und Sturm zu gebieten?

O zahme Kunst

der Zauberin,

die nur Balsamtränke noch braut!

Erwache mir wieder,

kühne Gewalt,

herauf aus dem Busen,

wo du dich bargst!

Hört meinen Willen,

zagende Winde!

Heran zu Kampf

und Wettergetös',

zu tobender Stürme

wüthendem Wirbel!

Treibt aus dem Schlaf

dies träumende Meer,

weckt aus dem Grund

seine grollende Gier;

zeigt ihm die Beute,

die ich ihm biete;

zerschlag' es dies trotzige Schiff,

des zerschellten Trümmer verschling's!

Und was auf ihm lebt, den wehenden Athem,

den lass' ich euch Winden zum Lohn!

BRANGÄNE (im äußersten Schreck, um Isolde sich bemühend)

0 weh! $!^{\text {IV }}$

Ach! Ach!

Des Übels, das ich geahnt!

Isolde! Herrin!

Theures Herz!

Was bargst du mir so lang?

Nicht eine Thräne

weintest du Vater und Mutter;

kaum einen Gruß

den Bleibenden botest du:

von der Heimat scheidend

kalt und stumm,
ISOLDA (selvaggiamente, come trasognata)

Razza degenere!

Indegna degli avi!

Dove, o Madre,

cedesti il potere

di comandare al mare e alla tempesta?

O ammansita

arte di maga,

che solo intruglia bevande di balsami!

A me ancor svégliati

0 ardito potere;

su dal mio cuore,

ove ti celasti!

Udite il mio volere

0 venti docili!

Suvvia alla lotta

e all'uragano!

Di rombanti procelle

al furente vortice!

Dal sonno scotete

questo mare che sogna;

dall'abisso svegliate,

l'irosa sua brama!

Mostrategli la preda

che io gli prometto!

Ch'egli sfracelli questo naviglio insolente,

e dello sfracello le rovine ingoi!

E quel che su di lui vive,

e il respiro che spira,

a voi venti in ricompensa lascio!

BRANGANIA (con estremo terrore, affaccendandosi intorno ad Isolda)

Guai!

Ahimè! Ahimè!

qual male ho presagito!

Isolda! Signora!

Cuor caro!

Che mi nascondesti così a lungo?

Non una lagrima

piangesti per tuo padre e tua madre;

appena un saluto

ai rimanenti porgesti.

Dalla patria partendo

fredda e muta;

\footnotetext{
IV GS: «Weh! O weh!».
} 
bleich und schweigend

auf der Fahrt,

ohne Nahrung,

ohne Schlaf,

starr und elend,

wild verstört: ${ }^{\mathrm{v}}$

wie ertrug ich, ${ }^{\text {vI }}$

so dich sehend

nichts dir mehr zu sein,

fremd vor dir zu stehn?

$\mathrm{O}$, nun melde

was dich müht!

Sage, künde

was dich quält.

Herrin Isolde,

trauteste Holde!

Soll sie wert sich dir wähnen,

vertraue nun Brangänen!

ISOLDE

Luft! Luft!

Mir erstickt das Herz.

Öffne! Öffne dort weit!

(Brangäne zieht eilig die Vorhänge in der Mitte auseinander)

\section{ZWEITE SZENE}

(Man blickt dem Schiff entlang bis zum Steuerbord, über den Bord hinaus auf das Meer und den Horizont. Um den Hauptmast in der Mitte ist Seevolk, mit Tauen beschäftigt, gelagert; über sie hinaus gewabrt man am Steuerbord Ritter und Knappen ebenfalls gelagert; von ihnen etwas entfernt Tristan, mit verschränkten Armen stehend, und sinnend in das Meer blickend; zu Füßen ibm, nachlässig ausgestreckt, Kurwenal. - Vom Maste her, aus der Höhe, vernimmt man wieder den Gesang des jungen Seemannes)

DER JUNGE SEEMANN

Frisch weht der Wind

der Heimat zu: -

mein irisch Kind,

wo weilest du? pallida e taciturna

lungo il viaggio;

senza cibo,

senza sonno;

rigida e misera

selvaggiamente turbata:

come sostenni

vedendoti così,

di non essere più niente per te,

e di starmi straniera al tuo cospetto?

Oh, dimmi dunque,

quel che t'angoscia!

Dimmi, palesa,

quel che ti tortura!

Madonna Isolda,

dolce, carissima!

Perch'ella creda per te ancor valere, confidati dunque a Brangania!

ISOLDA

Aria! Aria!

Mi soffoca il cuore!

Apri, spalanca costà!

(Brangania s'affretta a scostare le tende di mezzo.)

\section{SCENA SECONDA}

(La vista corre lungo la nave fino al timone, e, al di sopra del parapetto, al mare libero ed all'orizzonte. Intorno all'albero maestro, nel mezzo, i marinai stanno stesi per terra, affaccendati ai cordami; dietro di essi, a poppa, egualmente sdraiati, cavalieri e scudieri; un poco appartato da loro Tristano, in piedi, le braccia conserte, in atto di guardare meditabondo il mare; ai piedi di lui, negligentemente sdraiato, Kurwenal. Dall'alto dell'albero si sente nuovamente il canto del giovine marinaio)

\section{IL GIOVINE MARINAIO}

Fresco soffia il vento

verso la patria:

mia fanciulla d'Irlanda,

dove te ne stai?

\footnotetext{
v GS: «wild verstört, I starr und elend, -».

vI GS: «ich's,».
} 
Sind's deiner Seufzer Wehen, die mir die Segel blähen? -

Wehe! Wehe, du Wind!

Weh! Ach wehe, mein Kind!

ISOLDE (deren Blick sogleich Tristan fand, und starr auf ibn geheftet bleibt, dumpf für sich)

Mir erkoren, -

mir verloren, -

hehr und heil,

kühn und feig -:

Tod geweihtes Haupt! ${ }^{2}$

Tod geweihtes Herz!

(Zu Brangäne, unheimlich lachend)

Was hältst du von dem Knechte?

BRANGÄNE (ibrem Blicke folgend)

Wen meinst du?

ISOLDE

Dort den Helden
der meinem Blick
den seinen birgt,
in Scham und Scheue
abwärts schaut: -
g, wie dünkt er dich?

Sono gli aliti dei tuoi sospiri, che mi gonfiano le vele?

Ve' ve', o vento!

ve'! ah! ve', mia bimba!

ISOLDA (il cui sguardo ha subito scorto Tristano, ed è rimasto rigido e fisso su di lui, sordamente, tra sé)

Per me eletto,

per me perduto,

alto e puro,

prode e vile!

Capo sacro alla morte!

Cuor sacro alla morte!

(A Brangania, con riso sinistro)

Che ne pensi, di quel servo?

BRANGANIA (seguendo il suo sguardo)

Di chi parli?

ISOLDA

Di quell'eroe colà,
che al mio sguardo
il suo nasconde,
che pudico e timido,
guarda altrove:
mmi, che te ne pare?

${ }^{2}$ Introdotto da una lenta scala cromatica, che inanella una serie incalzante di motivi del Desiderio (es. $1 y$ ) sul crescendo in tremolo degli archi ( «Mir erkoren, -» ecc.), udiamo dalla voce di Isolde il motivo della Morte (Todesmotiv: es. 8) che incorpora (alla lettera $x$ dell'es. 8 ) il profilo di quello Fato (es. $2 x$ ). Motivo indissociabile dalla sua armonia, che trascolora nel timbro muovendosi tra legni e ottoni.

ESEMPIO 8 (bb. 318-324)

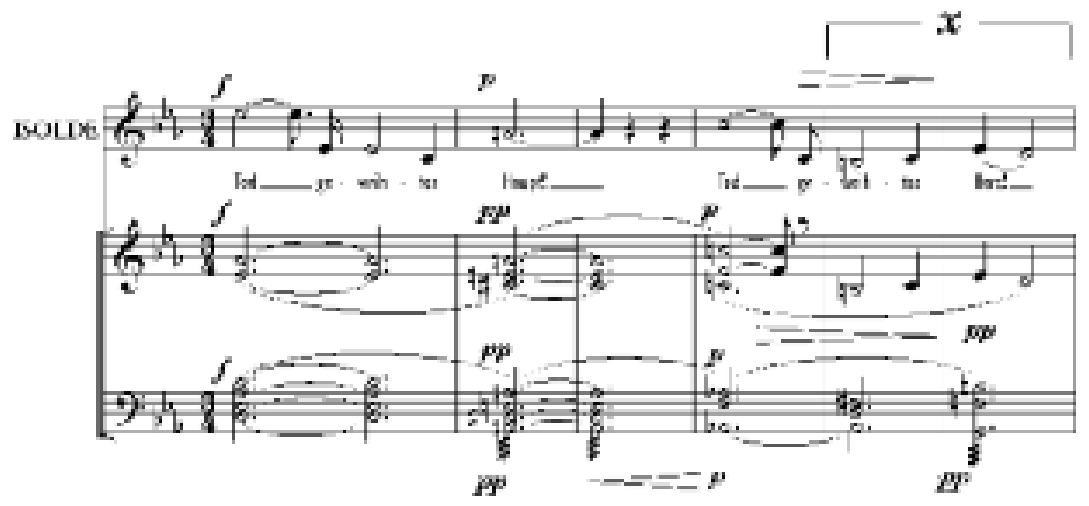

Sono le ultime battute controllate dalla tonalità della prima scena, do: intorbidata però (nella terza battuta del motivo) dall'inserzione di una triade maggiore sul La (tenuta in pianissimo da trombe, tromboni e timpani) che fa di questa musica il perfetto pendant alle enigmatiche parole della protagonista. 
BRANGÄNE

Frägst du nach Tristan, teure Frau, dem Wunder aller Reiche, dem hochgepriesnen Mann, dem Helden ohne Gleiche, des Ruhmes Hort und Bann?

ISOLDE (sie verhöhnend)

Der zagend vor dem Streiche sich flüchtet wo er kann, weil eine Braut er als Leiche für seinen ${ }^{\mathrm{VII}}$ Herrn gewann! -

Dünkt es dich dunkel, mein Gedicht?

Frag ihn denn selbst, den freien Mann,

ob mir zu nahn er wagt?

Der Ehren Gruß

und zücht'ge Acht

vergißt der Herrin

der zage Held,

daß ihr Blick ihn nur nicht erreiche -

den Helden ${ }^{\mathrm{VIII}}$ ohne Gleiche!

$\mathrm{O}$, er weiß

wohl warum! -

$\mathrm{Zu}$ dem Stolzen geh, meld ihm der Herrin Wort:

meinem Dienst bereit

schleunig soll er mir nahn.

BRANGÄNE

Soll ich ihn bitten,

dich zu grüßen?

ISOLDE

Befehlen ließ'

dem Eigenholde

Furcht der Herrin

ich, Isolde.

(Auf Isoldes gebieterischen Wink entfernt sich Brangäne, und schreitet verschämt dem Deck entlang dem Steuerbord $z u$, an den arbeitenden Seeleuten vorbei. Isolde, mit starrem Blicke ihr folgend, zieht sich rücklings nach dem Ruhebett zurück, wo sie sit-
BRANGANIA

Chiedi tu di Tristano, cara Signora?

Della meraviglia di tutti i reami, dell'uomo altamente celebrato?

Dell'eroe senza pari, tesoro e vanto di gloria?

ISOLDA (beffandola)

Colui che tremante di fronte al colpo

fugge, dov'egli può,

perché una sposa cadavere

al suo sovrano ha conquistato!

Oscuro ti sembra

il mio enigma?

Chiedi dunque a lui stesso, all'uomo libero

s'egli osa avvicinarsi a me?

Il saluto d'onore

ed il costumato omaggio,

oblia di fronte alla signora,

quel timido eroe;

purché lo sguardo di lei non raggiunga

l'eroe senza pari!

Oh, ei sa

sicuramente, perché!

A quel superbo va,

e la parola annunzia della sua signora!

Pronto al mio servizio, rapido a me s'accosti.

BRANGANIA

Devo pregarlo

di salutarti?

ISOLDA

Ho fatto comandare

al mio servo,

timore della sua signora;

io Isolda!

(Al cenno imperativo di Isolda, Brangania si allontana e si dirige vergognosa lungo la coperta verso poppa, passando avanti ai marinai che lavorano. Isolda, mentre la segue con lo sguardo fisso, retrocede, camminando all'indietro, verso il divano, do-

\footnotetext{
VII GS: «er seinem».

VIII GS: «Kühnen».
} 
zend während des Folgenden bleibt, das Auge unabgewandt nach dem Steuerbord gerichtet)

KURWENAL (der BRANGÄNE kommen sieht, zupft, obne sich zu erheben, Tristan am Gewande)

Hab Acht, Tristan!

Botschaft von Isolde.

TRISTAN (auffahrend)

Was ist? - Isolde? -

(Er faßt sich schnell, als Brangäne vor ibm anlangt und sich verneigt)

Von meiner Herrin? -

Ihr gehorsam

was zu hören

meldet höfisch

mir die traute Magd?

BRANGÄNE

Mein Herre Tristan,

euch $^{\mathrm{IX}} \mathrm{zu}$ sehen

wünscht Isolde,

meine Frau.

TRISTAN

Grämt sie die lange Fahrt, die geht zu End';

eh noch die Sonne sinkt,

sind wir am Land:

was meine Frau mir befehle, treulich sei's erfüllt.

BRANGÄNE

So mög' Herr Tristan

zu ihr gehn:

das ist der Herrin Will'.

TRISTAN

Wo dort die grünen Fluren

dem Blick noch blau sich färben,

harrt mein König

meiner Frau:

zu ihm sie zu geleiten

bald nah' ich mich der Lichten;

keinem gönnt' ich

diese Gunst.

BRANGÄNE

Mein Herre Tristan,

höre wohl: v'ella rimane a sedere durante la scena che segue, l'occhio fisso verso poppa)

KURWENAL (che scorge Brangania venire, senza alzarsi, tira Tristano per la veste)

Attento, Tristano!

Messaggio d'Isolda.

TRISTANO (trasalendo)

Che c'è? Isolda?...

(Si riprende rapidamente, allorché Brangania giunge presso di lui e s'inchina)

Dalla mia signora?

A lei obbediente,

che cosa udire

cortesemente annunzia

a me, la fedele ancella?

BRANGANIA

Signor mio Tristano,

di vedervi

desidera Isolda,

la mia signora.

TRISTANO

Se l'affligge il lungo viaggio,

esso volge alla fine;

prima ancora che tramonti il sole, siamo giunti a terra.

quel che mi comanda la mia sovrana

fedelmente s'adempia.

BRANGANIA

Che sire Tristano voglia dunque

da lei andare:

questo è il volere della mia signora.

TRISTANO

Laggiù dove le verdi pianure

ancora si colorano d'azzurro alla vista,

il mio re attende

la mia signora;

per accompagnarla a lui,

presto io m'accosterò alla luminosa:

a nessuno cederei

questo onore.

BRANGANIA

Mio signor Tristano, odi bene:

\footnotetext{
Ix GS: «dich».
} 
deine Dienste

will die Frau,

daß du zur Stell' ihr nahtest,

dort wo sie deiner harrt.

TRISTAN

Auf jeder Stelle

wo ich steh', getreulich dien' ich ihr,

der Frauen höchster Ehr'.

Ließ’ ich das Steuer

jetzt zur Stund',

wie lenkt' ich sicher den Kiel

zu König Markes Land?

BRANGÄNE

Tristan, mein Herre,

was höhnst du mich?

Dünkt dich nicht deutlich

die thör'ge Magd,

hör meiner Herrin Wort!

So hieß sie sollt' ich sagen: -

befehlen ließ'

dem Eigenholde

Furcht der Herrin

sie, Isolde.

KURWENAL (aufspringend)

Darf ich die Antwort sagen?

TRISTAN (ruhig)

Was wohl erwidertest du?

KURWENAL

Das sage sie

der Frau Isold'. -

Wer Kornwalls Kron'

und Englands Erb'

an Irland's Maid vermacht,

der kann der Magd

nicht eigen sein,

die selbst dem Ohm er schenkt.

Ein Herr der Welt

Tristan der Held!

Ich ruf's: du sag's, und grollten

mir tausend Frau Isolden.

(Da Tristan durch Gebärden ihm zu wehren sucht, und Brangäne entrüstet sich zum Weggehen wendet, singt Kurwenal der zögernd sich Entfernenden mit böchster Stärke nach:) i tuoi servigi

vuole la mia signora;

che tu subito a lei venga,

là dove ella t'attende.

TRISTANO

Ovunque

io sto,

fedelmente a lei servo,

ch'è onore supremo delle donne;

s'io lasciassi il timone,

così senz'altro,

come drizzerei sicuro il naviglio

alla terra di re Marke?

BRANGANIA

Tristano mio signore!

Perché ti beffi di me?

Se a te non par chiara

la semplicetta ancella,

odi la parola della mia signora!

Così ella mi comandò ch'io dovessi dire:

ha fatto comandare

al suo servo,

timore della sua signora:

ella, Isolda.

KURWENAL (balzando in piedi)

Posso io rispondere?

TRISTANO (tranquillo)

$\mathrm{E}$ che mai risponderesti tu?

KURWENAL

Questo dica ella

a madonna Isolda!

Colui che la corona di Cornovaglia

e l'eredità d'Inghilterra

lega alla fanciulla d'Irlanda, non può a quella fanciulla

esser servo,

che egli stesso dona allo zio.

Un dominatore del mondo

è l'eroe Tristano!

Io lo grido: tu dillo, e che se la prendano

con me mille madonne Isolde!

(Poiché Tristano con gesti cerca di farlo tacere, e Brangania, adirata, s'appresta a tornarsene, Kurwenal canta dietro di lei, che si allontana esitando, con tutta forza:) 
»Herr Morold zog

zu Meere her,

in Kornwall Zins zu haben;

ein Eiland schwimmt

auf ödem Meer,

da liegt er nun begraben:

sein Haupt doch hängt

im Iren-Land,

als Zins gezahlt

von Engeland.

Hei! unser Held Tristan!

Wie der Zins zahlen kann! «

(Kurwenal, von Tristan fortgescholten, ist in den Schiffsraum des Vorderdeckes binabgestiegen. Brangäne, in Bestürzung zu Isolde zurückgekehrt, schließt hinter sich die Vorhänge, während die ganze Mannschaft von außen den Schluß von Kurwenals Liede wiederholt)

ALLE MÄNNER

"Sein Haupt doch hängt

im Iren-Land,

als Zins gezahlt

von Engeland.

Hei! unser Held Tristan!

Wie der Zins zahlen kann!»

\section{DRITTE SZENE}

(Isolde und Brangäne allein, bei volkommen wieder geschlossenen Vorhängen. Isolde erhebt sich mit verzweiflungsvoller Wuthgebärde)

BRANGÄNE (ihr zu Füßen stürzend)

Weh! Ach, wehe!

Dies zu dulden!

ISOLDE (dem furchtbarsten Ausbruche nahe, schnell sich zusammenfassend)

Doch nun von Tristan:

genau will ich's vernehmen.

BRANGÄNE

Ach, frage nicht!

ISOLDE

Frei sag's ohne Furcht!

BRANGÄNE

Mit höf'schen Worten

wich er aus.
«Sire Morold se ne venne

per mare

in Cornovaglia, per aver tributo;

un'isola galleggia

sul mare deserto.

Colà egli ora giace sepolto!

Il suo capo tuttavia è appeso

in Irlanda

come tributo pagato

dall'Inghilterra:

viva l'eroe nostro Tristano,

come ei può pagare tributo!»

(Kurwenal, ripreso ancora da Tristano, è sceso sotto coperta; Brangania tornata smarrita da Isolda, tira $i$ cortinaggi dietro di sé, mentre dal di fuori tutto l'equipaggio riprende la fine della canzone di Kurwenal)

\section{TUTTI GLI UOMINI}

«Il suo capo tuttavia è appeso

in Irlanda,

come tributo pagato

dall'Inghilterra!

Viva l'eroe nostro Tristano,

com'ei può pagare tributo!»

\section{SCENA TERZA}

(Isolda e Brangania sole, con $i$ cortinaggi di nuovo ermeticamente chiusi. Isolda si alza con gesto disperato e furente)

BRANGANIA (precipitandosi ai suoi piedi)

Guai, ah! guai!

Questo dover soffrire!

ISOLDA (presso a prorompere nel modo piu terribile, ma subito rattenendosi)

[La risposta,] dunque, di Tristano!

Voglio conoscerla con precisione.

BRANGANIA

Ah! non domandare!

ISOLDA

Francamente dilla senza timore!

BRANGANIA

Con detti cortesi

se l'è cavata. 
ISOLDE

Doch als du deutlich mahntest?

BRANGÄNE

Da ich zur Stell'

ihn zu dir rief:

wo er auch steh',

so sagte er,

getreulich dien' er ihr,

der Frauen höchster Ehr';

ließ' er das Steuer

jetzt zur Stund',

wie lenkt er sicher den Kiel

zu König Markes Land?

ISOLDE (schmerzlich bitter)

»Wie lenkt' er sicher den Kiel

zu König Marke’s Land « -

(Grell und heftig)

den Zins ihm auszuzahlen,

den er aus Irland zog!

BRANGÄNE

Auf deine eignen Worte

als ich ihm die entbot,

ließ seinen Diener ${ }^{\mathrm{X}}$ Kurwenal -

ISOLDE

Den hab' ich wohl vernommen;

kein Wort, das mir entging.

Erfuhrest ${ }^{\mathrm{XI}}$ du meine Schmach, nun höre, was sie mir schuf. -

Wie lachend $\operatorname{sie}^{3}$

mir Lieder singen,
ISOLDA

Ma quando chiaramente l'ammonisti?

BRANGANIA

Quando immantinente

a te io lo chiamai:

dovunque egli stia,

così egli disse,

fedelmente egli serve a colei,

ch'è onore supremo delle donne;

s'egli lasciasse il timone,

così senz'altro,

come drizzerebbe sicuro il naviglio

alla terra di re Marke?

ISOLDA (dolorosamente amara)

"Come drizzerebbe sicuro il naviglio

alla terra di re Marke?»

(Con stridula violenza)

Per pagargli il tributo

ch'egli ha tratto d'Irlanda!

BRANGANIA

Alle tue proprie parole

quando io glie le riportai,

lasciò che il suo servitore Kurwenal!...

ISOLDA

Bene io l'ho udito:

nessuna parola, che mi sia sfuggita.

Poiché hai conosciuto la mia onta, odi ora quel che me l'ha procurata.

Come ridendo coloro

mi han cantato canzoni,

\footnotetext{
x GS: «Treuen».

xI GS: «Erfuhrst».
}

3 Nella scena precedente, il dileggio sgarbato di Kurwenal e della ciurma nei confronti di Isolde e della sua ancella ha introdotto un tipo di linguaggio nuovo: quello del rude Lied popolaresco in due parti che Kurwenal ha cantato dietro a Brangäne («Wer Kornwalls Kron'»; «Herr Morold zog»). Questo è appunto il linguaggio del 'giorno', nella sua forma più cinica e sguaiata. Da esso prendiamo nuovamente le distanze tornando con Brangäne dietro le cortine chiuse della tenda. Il racconto di Isolde si articola in due ampie sezioni, ben riconoscibili. Nella prima, Isolde rivela come avesse risanato il malconcio Tantris-Tristan: il «tema generatore» della musica come avrebbe detto Hubert - è qui un motivo nuovo, introdotto forte e sehr bewegt ("molto mosso») da legni e violini primi, il motivo di Tristan infermo (Motiv des siechen Tristan, $x$ nell'es. 9).

ESEMPIO 9 (bb. 602-604)

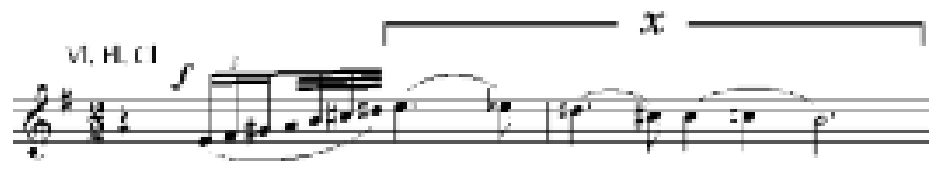


wohl könnt' auch ich erwidern: von einem Kahn, der klein und arm

an Irlands Küsten ${ }^{\mathrm{XII}}$ schwamm, darinnen krank ein siecher Mann elend im Sterben lag. Isoldes Kunst

ward ihm bekannt; mit Heil-Salben und Balsamsaft der Wunde, die ihn plagte, getreulich pflag sie da.

Der » Tantris «

mit sorgender List sich nannte, als » Tristan «

Isold' ihn bald erkannte, da in des Müß'gen Schwerte eine Scharte sie gewahrte, darin genau

sich fügt' ein Splitter, der einst im Haupt des Iren-Ritter, zum Hohn ihr heimgesandt, mit kund'ger Hand sie fand. -

Da schrie's mir auf aus tiefstem Grund; mit dem hellen Schwert ich vor ihm stund, an ihm, dem Über-Frechen, Herrn Morolds Tod zu rächen.

Von seinem Lager ${ }^{\text {XIII }}$

blickt' er her, nicht auf das Schwert, nicht auf die Hand, - anch'io potrei bene rispondere!

Di una navicella, che piccola e povera,

presso la costa d'Irlanda andava alla deriva;

entr'essa malato

un uomo consunto

miseramente giaceva presso alla morte.

L'arte d'Isolda

fu a lui conta;

con salutiferi unguenti,

e succhi balsamici

la ferita, che lo torturava,

fedelmente allora ella curò.

Il «Tantris»

con inquieta astuzia ei si nomò; come «Tristan»

Isolda subito lo riconobbe, che nella spada del languente una tacca ella scorse, entro la quale esattamente s'adattava una scheggia, cui un giorno nel capo

del cavaliere irlandese per smacco a lei inviato, con esperta mano ella aveva trovato.

Allora un grido mi si levò

dal mio più profondo!

Con la lucente spada

io stetti a lui dinnanzi;

su di lui, il temerario,

per vendicare la morte di Sire Morold.

Dal suo giaciglio

egli volse lo sguardo...

non sulla spada,

non sulla mano,...

segue nota 3

Il profilo cromatico discendente può essere sentito come un prolungamento del motivo del Dolore (es. $1 x$ ). L'intera pagina è solcata da questo motivo, condotto attraverso un'inesauribile gamma di sfumature di tempo e intensità. Verso la fine, una viola sola intarsia sehr ausdrucksvoll und zart («con molta dolcezza ed espressione») il motivo dello Sguardo (es. 2y), legandone indissolubilmente il significato all'incontro degli occhi dell'infermo e della sua guaritrice ( «er sah mir in die Augen»). Dopo una cadenza in mi, la seconda parte del racconto («Er schwur mit tausend Eiden»: «Ei giurò con mille giuramenti») attacca sehr feurig («con molto fuoco»), mentre Isolde avvampa di rabbia per la slealtà e l'infamia di Tristan, e urla propositi di vendetta: negli archi e nei legni gravi imperversa il motivo della Collera (es. 7).

XII GS: «Küste».

XIII GS: «Bette». 
er sah mir in die Augen.

Seines Elendes

jammerte mich;

das Schwert - ich ließ es ${ }^{\mathrm{xIV}}$ fallen!

Die Morold schlug, die Wunde, sie heilt' ich, daß er gesunde, und heim nach Hause kehre, mit dem Blick mich nicht mehr beschwere.

BRANGÄNE

O Wunder! Wo hatt' ich die Augen?

Der Gast, den einst ich pflegen half -?

ISOLDE

Sein Lob hörtest du eben; "Hei! Unser Held Tristan! « -

Der war jener traur'ge Mann. Er schwur mit tausend Eiden mir ew'gen Dank und Treue.

Nun hör' wie ein Held

Eide hält! -

Den als Tantris

unerkannt ich entlassen, als Tristan

kehrt $^{\mathrm{xv}}$ er kühn zurück; auf stolzem Schiff

von hohem Bord, Irlands Erbin begehrt ${ }^{\mathrm{XVI}}$ er zur Eh'

für Kornwall's müden König, für Marke, seinen Ohm.

Da Morold lebte, wer hätt' es gewagt uns je solche Schmach zu bieten?

Für der zinspflicht'gen ${ }^{\text {xVII }}$

Kornen Fürsten

um Irlands Krone zu werben?

Ach, ${ }^{\text {XVIII }}$ wehe mir!

Ich ja war's,

die heimlich selbst mi guardò negli occhi.

Della sua miseria

mi dolse;...

la spada... la lasciai cadere!

La ferita, quella che Morold inferse,

io la guarii, così che sano

in patria, al focolare tornasse,...

e della sua vista non più mi gravasse!

BRANGANIA

Oh meraviglia! I miei occhi dov'erano?

L'ospite, ch'io un giorno

aiutai a curare?

ISOLDA

La sua lode hai testé udita:

«Viva il nostro eroe Tristano»...

Era lui quell'infelice.

Ei giurò con mille giuramenti

a me eterna fede e gratitudine!

Ora odi come un eroe

i giuramenti tiene!

Colui che come Tantris

sconosciuto io congedai, come Tristano

indietro tornò arditamente su un superbo vascello

d'alto bordo;

l'erede d'Irlanda

egli chiedeva in nozze,

per lo stanco re di Cornovaglia,

per Marke suo zio.

Quando Morold viveva, chi avrebbe osato

mai proporci un'onta simile?

Per il tributario

principe di Cornovaglia

sollecitare la corona d'Irlanda!

Ah guai a me!

Io fui quella

che segretamente di mia mano

\footnotetext{
xIV GS: «das ließ ich».

xV GS: «kehrt'».

XVI GS: «begehrt'».

XVII GS: «zinspflichtigen».

XVIII GS: «O».
} 
die Schmach sich schuf!

Das rächende Schwert, statt es zu schwingen, machtlos ließ ich's fallen: nun dien' ich dem Vasallen.

BRANGÄNE

Da Friede, Sühn' und Freundschaft von Allen ward beschworen, wir freuten uns all' des Tags;

wie ahnte mir da, daß dir es Kummer schüf'?

ISOLDE

O blinde Augen!

Blöde Herzen!

Zahmer Muth, verzagtes Schweigen!

Wie anders prahlte

Tristan aus, was ich verschlossen hielt!

Die schweigend ihm

das Leben gab, vor Feindes Rache inn schweigend ${ }^{\mathrm{XIX}}$ barg; was stumm ihr Schutz zum Heil ihm schuf, mit ihr - gab er es preis.

Wie siegprangend, heil und hehr, laut und hell wies er auf mich: "das wär' ein Schatz, mein Herr und Ohm; wie dünkt' euch die zur Eh'?

Die schmucke Irin

hol' ich her;

mit Steg' und Wegen ${ }^{\mathrm{xx}}$

wohl bekannt,

ein Wink, ich flieg'

nach Irenland;

Isolde, die ist euer: mir lacht das Abenteuer! « -

Fluch dir, Verruchter!

Fluch deinem Haupt! mi costruii l'onta.

La spada della vendetta.

invece di vibrarla, impotente la lasciai cadere!

Ora io servo al vassallo!

BRANGANIA

Quando pace e conciliazione e amicizia

fu giurata da tutti,

noi tutti gioimmo di quel giorno;

come avrei allora presagito,

che t'avrebbe dato affanno?

ISOLDA

O occhi ciechi!

Cuori deboli!

Coraggio ammansito,

Pusillo tacere!

Come altrimenti andò

Tristano vantando,

quel ch'io tenevo celato!

Colei che in silenzio a lui

aveva donato la vita,

ed alla vendetta nemica

lui, in silenzio aveva sottratto;

quel che la di lei muta protezione

per sua salvezza a lui aveva creato...

insieme con lei egli ha dato altrui in balia!

Come magnificando vittoria,

florido e nobile,

ad alta e chiara voce

egli accennò a me:

"Questo sarebbe un tesoro,

o mio signore e zio;

come vi sembra ella per un matrimonio?

La graziosa Irlandese,

io vado a prendere;

di strade e sentieri

ben conscio,

un cenno, ed io volo

in Irlanda;

Isolda è vostra!...

Mi sorride l'avventura!»

Maledizione a te, infame!

Maledizione al tuo capo!

\footnotetext{
XIX GS: «schweigend ihn».

xx GS: «Wege».
} 
Rache! Tod!

Tod uns beiden!

BRANGÄNE (mit ungestümer Zärtlichkeit sich auf Isolde stürzend)

O Süße! Traute!

Teure! Holde!

Goldne Herrin!

Lieb' Isolde!

(Sie zieht Isolde allmählich nach dem Ruhebett)

Hör' mich! Komme!

Setz dich her! -

Welcher Wahn? $?^{\mathrm{Xx}}$

Welch eitles Zürnen?4

Wie magst du dich betören, nicht hell zu seh'n noch hören?

Was je Herr Tristan

dir verdankte,

sag, konnt' er's höher lohnen,

als mit der herrlichsten der Kronen?

So dient' er treu

dem edlen Ohm,

dir gab er der Welt

begehrlichsten Lohn:

dem eignen Erbe,

echt und edel,

entsagt' er zu deinen Füßen,

als Königin dich zu grüßen.

(Da Isolde sich abwendet, fährt sie immer traulicher fort)

Und warb er Marke

dir zum Gemahl,

wie wolltest du die Wahl doch schelten,

muß er nicht wert dir gelten?

Von edler Art

und mildem Mut,

wer gliche dem Mann

an Macht und Glanz?
Vendetta! Morte!

Morte a noi due!

BRANGANIA (precipitandosi su Isolda con impetuosa tenerezza)

O dolce! O diletta!

Cara! Piena di grazia!

Aurea signora!

Isolda amata!

(Ella trae lentamente Isolda verso il divano)

Odimi! Vieni!

Qui adàgiati!

Quale follia?

Quale collera vana?

Come puoi tu così turbarti,

da non veder più chiaro né udire?

Quel che mai Sire Tristano

a te fu debitore,

dimmi, poteva egli più altamente compensare,

che con la più superba delle corone?

Così egli fedelmente servì

al nobile zio;

ed a te egli donò del mondo

il più desiderato premio,

al proprio retaggio,

egli nobile e puro,

rinunzia ai tuoi piedi

per salutarti regina!

(Siccome Isolda torce il viso, prosegue in tono sempre più confidenziale)

E s'egli ottenne Marke

a te per sposo,

come vorresti tu mai la scelta biasimare,

o può Marke non sembrarti degno?

Di nobile schiatta,

ed animo mite,

chi a lui potrebbe assomigliare

in potenza e splendore?

xxI GS: «Hör' mich! Komme! I Setz dich her! - I (Sie zieht Isolde allmäblich nach dem Ruhebett.) I Welcher Wahn?».

${ }^{4}$ Dalla furia di Isolde e dal suo motivo della Collera (es. 7) approdiamo al suadente «Welcher Wahn?» di Brangäne attraverso un passaggio che dimostra tutta la capacità wagneriana di «mediare e legare intimamente tra loro [Vermittelung und innige Verbindung] tutti i momenti della transizione fra gli stati estremi» (come scrive Wagner nella nota lettera a Mathilde Wesendonck sull'arte della transizione del 29 ottobre 1859). In un rassicurante e sommesso Mib, ora l'ancella si assume l'ingrato compito di placare Isolde e di "presentarle la situazione in una luce diversa» (così l'abbozzo in prosa del 1857). Dopo le convulsioni della sua signora, il canto di Brangäne si distende in periodi di otto battute, tracciando simmetrie insinuanti e carezzevoli quanto i gesti dell'ancella. 
Dem ein hehrster Held

so treulich dient, wer möchte sein Glück nicht teilen,

als Gattin bei ihm weilen?

ISOLDE (starr vor sich bin blickend)

Ungeminnt

den hehrsten Mann

stets mir nah zu sehen, -

wie könnt' ich die Qual bestehen!

BRANGÄNE

Was meinst ${ }^{\mathrm{xxII}}$ du Arge?

Ungeminnt? -

(Sie nähert sich ibr wieder schmeichelnd und kosend)

Wo lebte der Mann,

der dich nicht liebte?

Der Isolden ${ }^{\text {xxIII }}$ säh',

und in Isolden

selig nicht ganz verging'?

Doch, der dir erkoren,

wär' er so kalt,

zög' ihn von dir

ein Zauber $a b$,

den bösen wüßt' ich

bald zu binden;

ihn bannte der Minne Macht.

(Mit geheimnißvoller Zutraulichkeit ganz nahe zu Isolden $)^{5}$

Kennst du der Mutter

Künste nicht?

Wähnst du, die alles
Di colui, al quale il più nobile degli eroi

così fedelmente serve, chi rifiuterebbe di partecipare alla fortuna, e vicino a lui di vivere qual sposa?

ISOLDA (guardando fissa e trasognata innanzi a sé)

Non amata,

il più nobile degli uomini

vedere sempre presso di me:

come potrei questo supplizio tollerare?

BRANGANIA

Cattiva, che dici?

Non amata?

(Si avvicina a lei vezzeggiandola e carezzandola)

Dove vivrebbe mai un uomo,

che non t'amasse?

che vedesse Isolda,

ed in Isolda

beatamente tutto non si perdesse?

Pure, se colui che ti è stato scelto

così gelido fosse,

e da te lo distogliesse

una magia,

il maligno saprei io

subito incatenare;

la potenza dell'amore lo incanterebbe.

(Con misteriosa confidenza, vicinissima a Isolda)

Non conosci di tua madre

le arti?

Pensi tu, che colei la quale tutto

\footnotetext{
xXII GS: «wähnst».

XxIII GS: «Isolde».
}

5 L'allusione misteriosa di Brangäne alle arti magiche della madre di Isolde introduce una brusca cesura nella musica. Dopo un fremito dei violoncelli soli, i legni reintroducono il motivo del Desiderio in un contesto e in una tonalità ( $\mathrm{Sol}_{3} \mathrm{HLa}_{3}-\mathrm{La \#}_{3}-\mathrm{Si}_{3}$ ) che sono quelle delle prime battute del Vorspiel (ossia l'es. 1). Da questo momento, il preludio si reimpossessa della scena musicale: l'orchestra moltiplica i riferimenti alle pagine introduttive del Tristan, mescolandole a una cupa pulsazione dei timpani e interpolandovi il motivo della Morte (es. 8: «Rache für den Verrat, $-»$ : «Vendetta per il tradimento...»). Tra i molti lacerti riconoscibili del preludio ci sono anche la cadenza d'inganno con appoggiatura del motivo del Fato (es. 2), il poderoso crescendo dell'es. $3 x$ nei fagotti e negli ottoni gravi («Du irrst, ich kenn' ihn besser») e perfino l'es. 4 («So reihte sie die Mutter»). L'improvviso richiamo della ciurma dall'esterno («Ho! he! ha! he!») spezza però questo esercizio di memoria musicale e introduce il canto gioioso dei marinai (il primo di Wagner dai tempi lontani dell'Olandese volante), che si appropria anche del «Frisch weht der Wind» della prima scena (es. 6), piegato alla misura di $\mathbf{8}$. La chiassosa parentesi però non dura molto: rispedito Kurwenal a convocare Tristan, Isolde tornerà a occuparsi dei filtri materni con Brangäne. Così farà anche l'orchestra: nel momento in cui Isolde ritorce violenta contro la «malfida ancella» le sue stesse parole («Kennst du der Mutter»), il motivo del Desiderio si ripresenta nella forma dell'es. 1. Seguito, ancora una volta, da brandelli del preludio. 
klug erwägt,

ohne Rat in fremdes Land

hätt' sie mit dir mich entsandt?

ISOLDE (düster)

Der Mutter Rat

gemahnt mich recht;

willkommen preis' ich

ihre Kunst: -

Rache für den Verrat, -

Ruh in der Not dem Herzen! -

Den Schrein dort bring mir her.

BRANGÄNE

Er birgt, was heil dir frommt.

(Sie holt eine kleine goldene Trube herbei, öffnet sie, und deutet auf ibren Inbalt)

So reihte sie die Mutter, die mächt'gen Zaubertränke.

Für Weh und Wunden

Balsam hier;

für böse Gifte

Gegen-Gift: -

(Sie zieht ein Fläschchen hervor)

den hehrsten Trank,

ich halt' ihn hier.

ISOLDE

Du irrst, ich kenn' ihn besser;

ein starkes Zeichen

schnitt ich inm ein.

(Sie ergreift ein Fläschchen und zeigt es)

Der Trank ist's, der mir taugt! ${ }^{\mathrm{xxIV}}$

BRANGÄNE (entsetzt zurückweichend)

Der Todestrank!

(Isolde hat sich vom Rubebett erhoben, und vernimmt jetzt mit wachsendem Schrecken den Ruf des Schiffsvolkes:)

SCHIFFSVLK (außen)

»Ho! he! ha! ${ }^{\mathrm{Xxv}}$ he!

Am Untermast

die Segel ein!

"Ho! he! ha! ${ }^{\mathrm{xxv}}$ he!

ISOLDE

Das deutet schnelle Fahrt.

Weh' mir! Nahe das Land! accortamente pondera, senza consiglio in paese straniero mi avrebbe con te inviata?

ISOLDA (cupa)

Il consiglio di mia madre

bene mi ammonisce;

benenuta io lodo

la sua arte:...

Vendetta per il tradimento...

Pace ad un cuore in angoscia!...

Portami qui lo scrigno!

BRANGANIA

Esso contiene quel che ti porterà salute.

(Ella va a prendere un piccolo scrigno d'oro, lo apre

ed accenna al suo contenuto)

Così tua madre li ha disposti

i potenti magici filtri.

Per fiere ferite,

qui balsamo;

per violenti veleni,

contraveleni.

(Estrae una piccola fiala)

Il più nobile filtro, eccolo qui.

ISOLDA

Tu sbagli, io lo conosco meglio;

un profondo segno

io v'ho inciso.

(Ella prende una fialetta e la mostra)

Questo è il filtro che vale per me!

BRANGANIA (retrocede spaventata)

Il filtro della morte!

(Isolda si è alzata dal divano ed intende con crescente terrore il grido della ciurma:)

LA CIURMa (dal di fuori)

«Ho! he! ha! he!

All'artimone

raccogliete le vele!

Ho! he! ha! he!»

ISOLDA

Questo significa rapido viaggio.

Guai a me! Vicina è la terra!

xxIV GS: «Der Trank ist's, der mir frommt. I (Sie ergreift ein Fläschchen und zeigt es)».

xxv GS: «He! ha! ho!». 


\section{VIERTE SZENE}

(Durch die Vorhänge tritt mit Ungestüm Kurwenal herein.)

KURWENAL

Auf, auf! Ihr Frauen!

Frisch und froh!

Rasch gerüstet!

Fertig nun, hurtig und flink!

(Gemessener)

Und Frau Isolden

sollt' ich sagen

von Held Tristan,

meinem Herrn: -

vom Mast der Freude Flagge,

sie wehe lustig ins Land;

in Markes Königschlosse

mach' sie ihr Nah'n bekannt.

Drum Frau Isolde

bät' er eilen,

fürs Land sich zu bereiten, daß er sie könnt' geleiten.

ISOLDE (nachdem sie zuerst bei der Meldung in Schauer zusammengefahren, gefaßt und mit Würde)

Herrn Tristan bringe

meinen Gruß,

und meld ihm was ich sage. -

Sollt' ich zur Seit' ihm gehen,

vor König Marke zu stehen,

nicht möcht' es nach Zucht

und Fug geschehn,

empfing' ich Sühne

nicht zuvor

für ungesühnte Schuld:

drum such' er meine Huld.

(Kurwenal macht eine trotzige Gebärde. Isolde fährt mit Steigerung fort)

Du merke wohl

und meld es gut! -

Nicht woll ${ }^{\text {XxvI }}$ ich mich bereiten,

ans Land ihn zu begleiten;

(Sich mäßigend)

nicht werd' ich zur Seit' ihm gehen,

vor König Marke zu stehen,
SCENA QUARTA

(Attraverso i cortinaggi entra Kurwenal impetuosamente.)

KURWENAL

Su! Su! Donne!

Vive e vispe!

Presto, preparatevi!

$\mathrm{Su}$, pronte, agili e preste!

(Moderandosi)

Ed a madonna Isolda

dovrei dire,

da parte di sire Tristano,

mio signore:

dall'albero il gagliardetto di gioia

gaiamente sventoli verso terra;

nel reale castello di Marke,

renda noto l'avvicinarsi di lei.

E però madonna Isolda

egli pregherebbe d'affrettarsi, ad apprestarsi allo sbarco, così che la possa accompagnare.

ISOLDA (trasalisce dapprima all'annunzio rabbrividendo; poi riprendendosi e con dignità)

A sir Tristano porta

il mio saluto,

ed annunziagli quel che io dico.

Se io dovessi camminargli al fianco,

per presentarmi a re Marke,

non potrebbe questo secondo il costume

e la legge avvenire,

s'io non ricevessi soddisfazione

prima,

per una colpa non ancora espiata:...

e però, cerchi la mia clemenza.

(Kurwenal fa un gesto di sfida. Isolda continua con maggior forza)

Tu intendi bene,

e riferisci bene!

Non io mi preparerò

ad accompagnarlo nello sbarco;

(Contenendosi)

non io gli camminerò al fianco,

per presentarmi a re Marke,

XxvI GS: «Wollt'». 
begehrte Vergessen

und Vergeben

nach Zucht und Fug

er nicht zuvor

für ungebüßte Schuld: -

die böt' ihm meine Huld.

KURWENAL

Sicher wißt,

das sag' ich ihm:

nun harrt, wie er mich hört!

(Er geht schnell zurück.)

ISOLDE (eilt auf Brangäne zu und umarmt sie heftig)

Nun leb wohl, Brangäne!

Grüß mir die Welt,

grüße mir Vater und Mutter!

BRANGÄNE

Was ist? ${ }^{\text {xvII }}$ Was sinnst du?

Wolltest du fliehn?

Wohin soll ${ }^{\mathrm{XxVIII}}$ ich dir folgen?

ISOLDE (schnell gefaßt)

Hörtest du nicht?

Hier bleib' ich;

Tristan will ich erwarten. -

Getreu ${ }^{x x I x}$ befolg,

was ich befehl':

den Sühne-Trank

rüste schnell, -

du weißt, den ich dir wies? ?xx $^{\mathrm{xx}}$

BRANGÄNE

Und welchen Trank?

ISOLDE (entnimmt dem Schreine das Fläschchen)

Diesen Trank!

In die goldne Schale

gieß ihn aus;

gefüllt faßt sie ihn ganz.

BRANGÄNE (voll Grausen das Fläschchen empfangend)

Trau' ich dem Sinn? ISOLDE

Sei du mir treu! se non chiederà oblio

e perdono,

secondo il costume e la legge,

egli prima,

per la colpa non espiata:...

quelli gli offrirebbe la mia grazia.

KURWENAL

Sappiate di certo, questo gli rapporterò;

ora attendete, com'egli mi ascolterà!

(Si ritira in fretta)

ISOLDA (s'affretta da Brangania e la abbraccia con violenza)

Ed ora addio, Brangania!

Salutami il mondo,

salutami mio padre e mia madre!

BRANGANIA

Che c'è? Che mediti?

Vorresti fuggire?

Dove debbo io seguirti?

ISOLDA (si riprende rapidamente)

Non hai udito?

Qui io rimango:

voglio attender Tristano.

Fedelmente obbedisci

a quel che ti comando;

la bevanda della riconciliazione

rapida appresta;

tu sai, quella che ti ho mostrata?

BRANGANIA

E quale filtro?

ISOLDA (toglie la fialetta dallo scrigno)

Questo!

Nella coppa d'oro

versalo:

piena, lo contiene tutto.

BRANGANIA (piena d'orrore, ricevendo la fiala)

Debbo credere alla mia vista?

ISOLDA

Siimi fedele!

\footnotetext{
XXVII GS: «ist's?».

xxVIII GS: «sollt'».

xxIx GS: «Treu».

xxx GS: «wies.».
} 


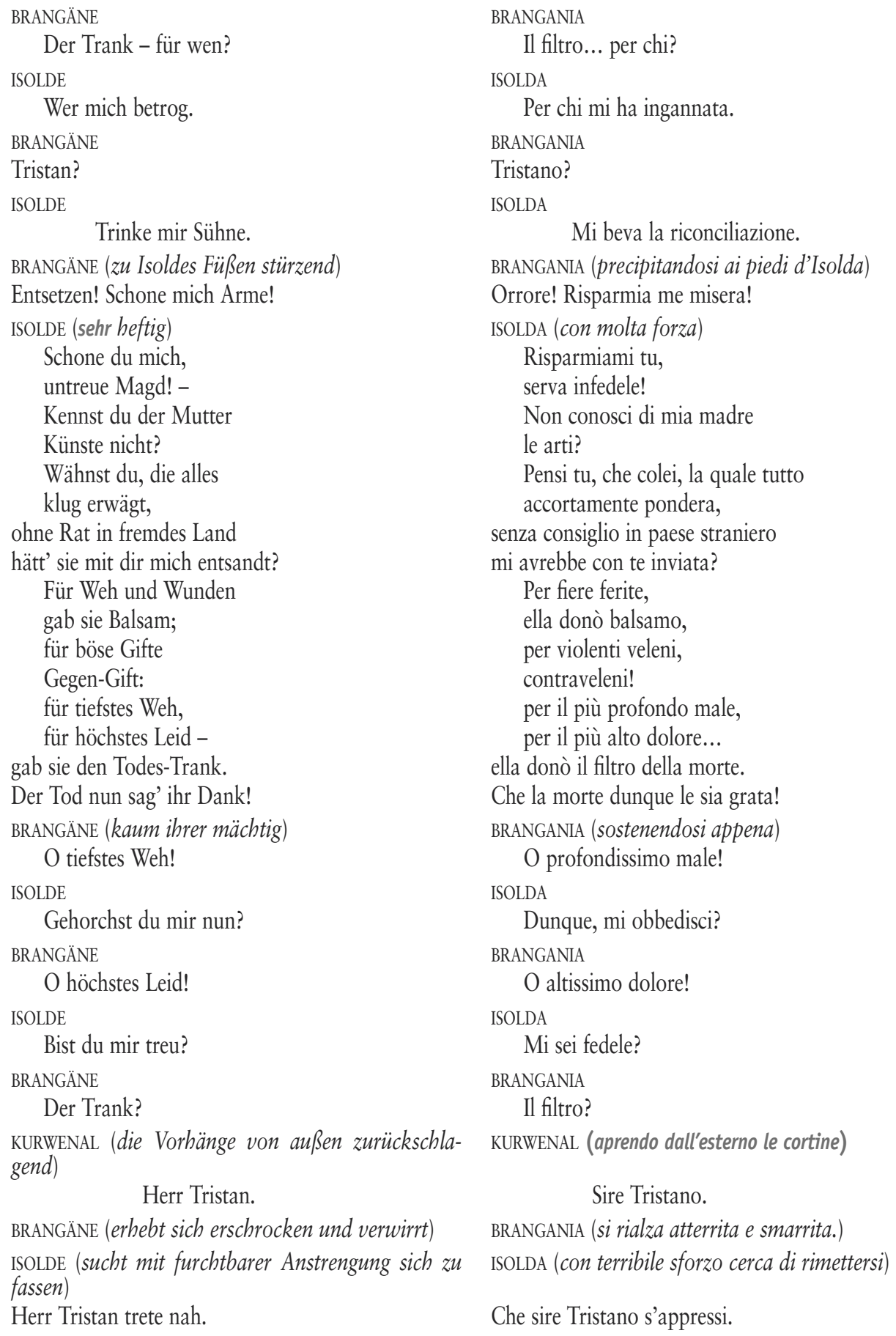




\section{FÜNFTE SZENE}

(Kurwenal geht wieder zurück. Brangäne, kaum ihrer mächtig, wendet sich in den Hintergrund. Isolde, ibr ganzes Gefübl zur Entscheidung zusammenfassend, schreitet langsam, mit großer Haltung, dem Rubebette zu, auf dessen Kopfende sich stützend sie den Blick fest dem Eingange zuwendet. Tristan tritt ein, und bleibt ehrerbietig am Eingange stehen. Isolde ist mit furchtbarer Aufregung in seinen Anblick versunken. - Langes Schweigen) ${ }^{6}$

\section{SCENA QUINTA}

(Kurwenal nuovamente si ritira. Brangania, sostenendosi appena, si volge verso il fondo. Isolda, raccogliendo tutte le forze per l'istante decisivo, s'avvia lentamente, e con gran contegno verso il divano, ad una delle estremità del quale s'appoggia, mentre volge lo sguardo fisso verso l'ingresso. Tristano entra e rimane rispettosamente in piedi sulla soglia. - Isolda, spaventevolmente agitata, s'immerge nella sua vista. - Lungo silenzio)

${ }^{6}$ La fissità e il silenzio di questa apertura di scena sono movimentati e riempiti da una delle invenzioni tematiche più memorabili del Wagner tristaniano. Per due volte corni e legni suonano un disegno di quattro note contraddistinto da una drammatica escursione dinamica (piano < fortissimo: $x$ dell'es. 10); per altrettante volte, gli archi rispondono con una serie di accordi staccati e molto pesanti, cui contribuisce la quarta corda dei violini (motivo delle Usanze: Sittemotiv, y dell'es. 10).

ESEMPIO 10 (bb. 1318-1324)
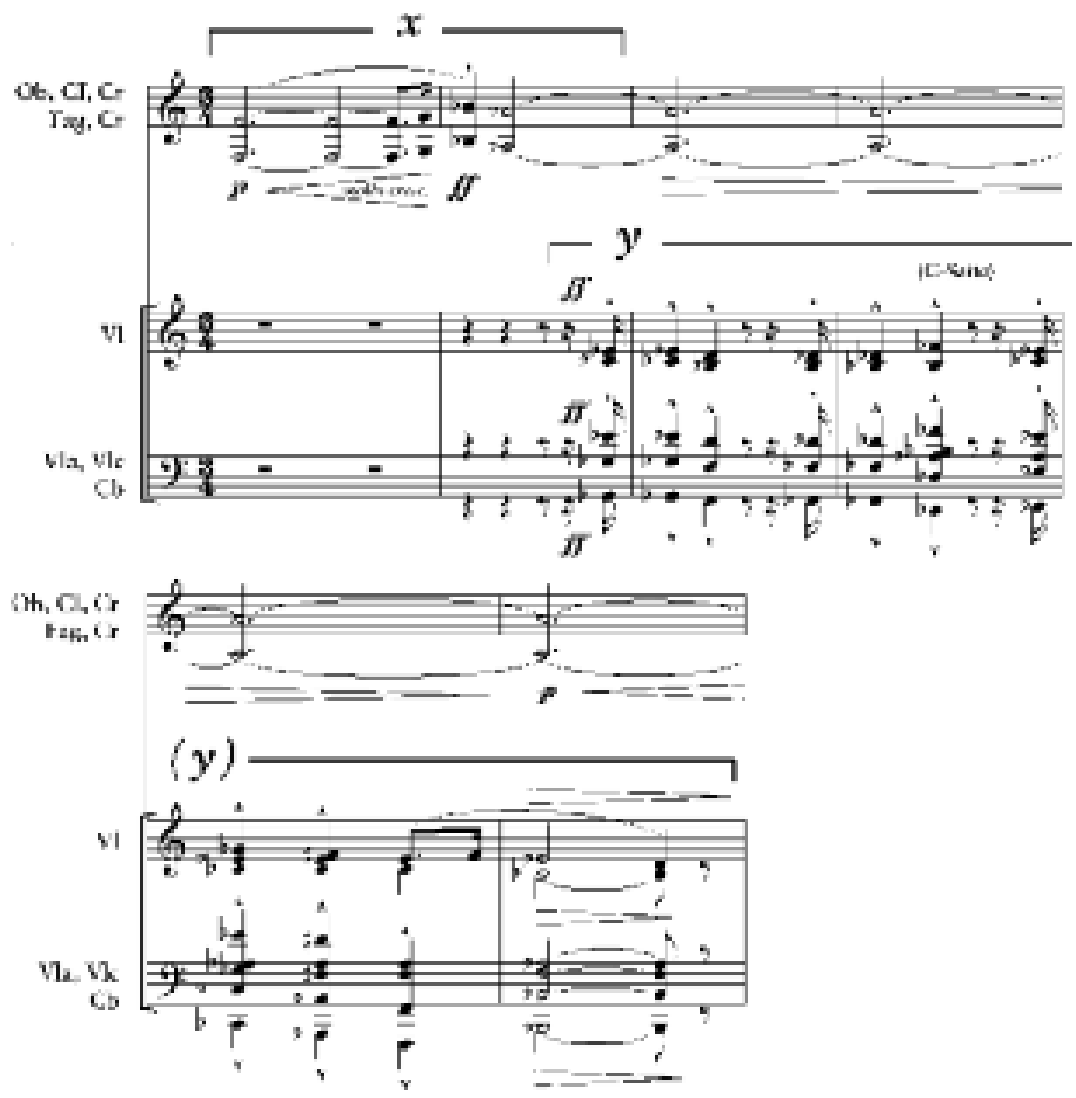

Il primo (es. 10x) è una variante del motivo del Fato (es. $2 x$ ); il secondo (es. 10y) ricorda invece il contorno della seconda frase del motivo dello Sguardo (che nel preludio coinvolgeva a sua volta la quarta corda dei violini: 
TRISTAN

Begehrt, Herrin,

was ihr wünscht.

ISOLDE

Wüßtest du nicht

was ich begehre,

da doch die Furcht

mir's zu erfüllen

fern meinem Blick dich hielt?

TRISTAN

Ehr-Furcht

hielt mich in Acht.

ISOLDE

Der Ehre wenig

botest du mir:

mit offnem Hohn

verwehrtest du

Gehorsam meinem Gebot.

TRISTAN

Gehorsam einzig

hielt mich in Bann.

ISOLDE

So dankt' ich Geringes

deinem Herrn,

riet dir sein Dienst

Un-Sitte

gegen sein eigen Gemahl?

TRISTAN

Sitte lehrt

wo ich gelebt:

zur Brautfahrt
TRISTANO

Comandate, signora,

quel ch'è vostro desiderio.

ISOLDA

Non sai tu forse

quel ch'io desidero;

da poi che la paura

di adempierlo

ti ha tenuto lontano dalla mia vista?

TRISTANO

Rispetto

mi ha trattenuto.

ISOLDA

Poco onore

m'hai reso;

con aperto dispregio

rifiutasti

obbedienza al mio comando.

TRISTANO

Obbedienza soltanto

m'ha tenuto appartato.

ISOLDA

Così poco dovrei io essere grata

al tuo signore,

se il suo servizio t'ha indotto

a mal costume

verso la sua stessa consorte?

TRISTANO

Il costume insegna

dove io ho vissuto;

nel condurla allo sposo,

segue nota 6

es. $2 z$ ). Le due idee punteggiano la contesa iniziale sulle usanze, nella quale la deferenza di Tristan («Begehrt, Herrin») viene messa a dura prova dalle obiezioni di Isolde, che evoca infine il debito di sangue sulle armonie del motivo della Morte (es. 8) («Blut-Schuld»). Una versione mitigata del motivo della Collera (es. 7) ci parla dei sentimenti rattenuti della donna: ora, sulle note del motivo di Tristan infermo (es. 9), Isolde rievoca la stanza nella quale l'uomo giaceva malato in suo potere, e il voto che le resta da adempire ( "Da stand er herrlich»: «Là egli si drizzava splendido»). Nel resto della scena, l'es. 9 continuerà a giocare un ruolo da contraltare lirico dell'es. $10 x$. Il proposito di vendetta di Isolde contro l'uccisore del principe irlandese che le era destinato («Rache für Morold») riporta infatti in orchestra il motivo iniziale dei corni (es. 10x) in una versione particolarmente bellicosa $\mathrm{e}$ in accelerando. Ma gli accenti guerreschi cedono ancora una volta al timbro degli archi, e al languido cromatismo del motivo di Tristan infermo: Isolde offre il brindisi alla pace in un assolo di intensità elevatissima ( «Wie sorgt' ich schlecht»). Siamo al punto culminante della scena, annunciato a ripetizione dagli ottoni gravi con il motivo dell'es. $3 x$ sul tremolo degli archi: mentre Brangäne prepara esitante la coppa, dall'esterno irrompe di nuovo il suono delle voci della ciurma, che produce però qui un effetto allucinato. Sulle note di un'ultima trasformazione del motivo di Tristan infermo, Isolde rinnova con successo la sua offerta di riconciliazione ( «Du hörst den Ruf?»). 
der Brautwerber meide fern die Braut.

ISOLDE

Aus welcher Sorg'?

TRISTAN

Fragt die Sitte!

ISOLDE

Da du so sittsam, mein Herr Tristan, auch einer Sitte sei nun gemahnt: den Feind dir zu sühnen, soll er als Freund dich rühmen.

TRISTAN

Und welchen Feind? ISOLDE

Frag' deine Furcht!

Blut-Schuld

schwebt zwischen uns.

TRISTAN

Die ward gesühnt.

ISOLDE

Nicht zwischen uns.

TRISTAN

Im offnen Feld

von $^{\mathrm{xXX}}$ allem Volk

ward Ur-Fehde geschworen.

ISOLDE

Nicht da war's, wo ich Tantris barg, wo Tristan mir verfiel.

Da stand er herrlich, hehr und heil; doch was er schwur, das schwor ${ }^{\mathrm{XxXII}}$ ich nicht: zu schweigen hatt' ich gelernt.

$\mathrm{Da}$ in stiller Kammer

krank er lag, mit dem Schwerte stumm ich vor ihm stund, schwieg - da mein Mund, bannt' - ich meine Hand,

XXXI GS: «VOr».

XxXII GS: «schwur». colui che ha fatto la domanda si tenga lontano dalla fidanzata.

ISOLDA

Per qual timore?

TRISTANO

Domandate al costume!

ISOLDA

Poiché così [sei] costumato, mio signor Tristano, un altro costume sia qui ricordato: per riconciliarti il nemico, questi deve poterti celebrare amico. TRISTANO

E quale nemico?

ISOLDA

Chiedi alla tua paura!

Faida di sangue, si libra tra noi.

TRISTANO

Fu cancellata.

ISOLDA

Non tra noi.

TRISTANO

In campo aperto, davanti a tutto il popolo, fu giurata rinunzia alla vendetta.

ISOLDA

Non là avvenne dov'io Tantris nascosi, dove Tristan cadde in mio potere.

Là egli si drizzava splendido florido e nobile; pure quel ch'egli giurò non lo giurai io:

a tacere io avevo appreso.

Quando nella camera silenzioso malato egli giacque, muta, con la spada avanti a lui io stetti: tacque allora la mia bocca, e fermai la mia mano,... 
doch was einst mit Hand

und Mund ich gelobt,

das schwur ich schweigend zu halten.

Nun will ich des Eides walten.

TRISTAN

Was schwurt ihr, Frau?

ISOLDE

Rache für Morold.

TRISTAN

Müht euch die?

ISOLDE

Wagst du zu höhnen (xxxIII $^{\text {}}$

Angelobt war er mir,

der hehre Irenheld;

seine Waffen hatt' ich geweiht,

für mich zog er zum ${ }^{\text {xxIv }}$ Streit.

$\mathrm{Da}$ er gefallen,

fiel meine Ehr';

in des Herzens Schwere

schwur ich den Eid,

würd' ein Mann den Mord nicht sühnen,

wollt' ich Magd mich dess' erkühnen. -

Siech und matt

in meiner Macht, warum ich dich da nicht schlug? ${ }^{\mathrm{xxxv}}$

Das sag dir selbst mit leichtem Fug. ${ }^{\text {XxxvI }}$

Ich pflag des Wunden,

daß den heil Gesunden

rächend schlüge der Mann,

der Isolden ihn abgewann. -

Dein Los nun selber

magst du dir sagen:

da die Männer sich all' ihm vertragen,

wer muß nun Tristan schlagen?

TRISTAN (bleich und düster)

War Morold dir so wert, nun wieder nimm das Schwert, und führ es sicher und fest, (Er reicht ihr sein Schwert dar) daß du nicht dir's entfallen läßt. ${ }^{\text {xxvII }}$ pure quel che un giorno con la mano

e con la bocca giurai,

questo in silenzio giurai di mantenere.

Ora io voglio adempiere al mio giuramento.

TRISTANO

Che giuraste, madonna?

ISOLDA

Vendetta per Morold.

TRISTANO

Vi sta a cuore?

ISOLDA

Osi schernirmi?

A me fidanzato era

il nobile eroe d'Irlanda;

le sue armi io avevo consacrato;

per me egli traeva a battaglia.

Com'egli cadde

cadde il mio onore;

nell'angoscia del mio cuore

giurai il giuramento:

se nessun uomo avesse vendicato quella morte,

io, fanciulla, l'avrei osato.

Malato, languente

in mio potere,

perché allora io non ti colpii?

Te lo dirò io stessa, con aperta ragione.

Io mi curai del ferito,

affinché sano e integro,

lo colpisse con la sua vendetta l'uomo, che lo togliesse di mano a Isotta.

La tua sorte, dunque, tu stesso

puoi dirti:

poiché tutti gli uomini con lui vivono d'accordo, chi deve ormai colpire Tristano?

TRISTANO (pallido e cupo)

Se Morold t'era così caro,

prendi dunque nuovamente la sua spada,

e vibrala salda e sicura,

(Le porge la sua spada)

così che non te la lasci sfuggire.

\footnotetext{
xxxIII GS: «mir Hohn? -».

xxxIV GS: «in».

xxxv GS: «schlug,».

xxxvI GS: «dir mit leichtem Fug: -».

xxxvII GS: «daß du nicht dir's entfallen läßt. I (Er reicht ihr sein Schwert hin)».
} 
ISOLDE

Wie sorgt' ich schlecht um deinen Herren; was würde König

Marke sagen, erschlüg' ich ihm den besten Knecht, der Kron' und Land ihm gewann, den allertreusten Mann?

Dünkt dich so wenig was er dir dankt, bringst du die Irin ihm als Braut, daß er nicht schölte, schlüg' ich den Werber, der Urfehde-Pfand

so treu ihm liefert zur Hand? -

Wahre dein Schwert!

Da einst ich's schwang,

als mir die Rache

im Busen rang,

als dein messender Blick

mein Bild sich stahl,

ob ich Herrn Marke

taug' als Gemahl:

das Schwert - da ließ ich's sinken.

Nun laß uns Sühne trinken!

(Sie winkt Brangäne. Diese schaudert zusammen, schwankt und zögert in ihrer Bewegung. Isolde treibt sie durch gesteigerte Gebärde an. Als Brangäne zur Bereitung des Trankes sich anläßt, vernimmt man den Ruf des)

SCHIFFSVOLKES (von außen)

Ho! he! ha! he!

Am Obermast

die Segel ein!

Ho! he! ha! he!

TRISTAN (aus finsterem Brüten auffahrend)

Wo sind wir?

ISOLDE

Hart am Ziel.

Tristan, gewinn' ich Sühne?

Was hast du mir zu sagen?

TRISTAN (düster)

Des Schweigens Herrin

heißt mich schweigen:
ISOLDA

Come male io provvederei

al tuo signore;

che direbbe re

Marke,

se io gli uccidessi

il miglior suo servo,

che terra e corona gli ha guadagnato,

l'uomo suo più fedele?

Ti sembra così poco

quel ch'egli ti deve,

da poi che porti l'irlandese

a lui fidanzata;

che egli non avesse a prendersela,

s'io gli uccidessi colui che ha chiesto la mia mano,

ed in pegno della conciliazione

così fedelmente in sua mano consegna?

Conserva la tua spada!

Giacché io la brandii

quando a me la vendetta

lottava nel cuore:...

quando il tuo sguardo scrutatore

segretamente s'appropriava la mia immagine,

per giudicare. se io a re Marke

valessi quale sposa:...

quella spada... allora, io la lasciai cadere.

Dunque beviamo la riconciliazione!

(Ella accenna a Brangania, la quale trasalisce, barcolla e indugia nel suo muoversi. Isolda la incita con gesto piu imperioso. Nel momento in cui Brangania si accinge a preparare la bevanda, si ode il grido della)

CIURMA (dal di fuori)

Ho! he! ha! he!

All'albero maestro

raccogliete le vele!

Ho! he! ha! he!

TRISTANO (riscotendosi da una cupa meditazione)

Dove siamo?

ISOLDA

Ormai alla meta.

Tristano, otterrò la conciliazione?

Che hai da dirmi?

TRISTANO (cupamente)

La signora del silenzio

m'ordina il silenzio:... 
fass' ich was sie verschwieg, verschweig' ich was sie nicht faßt.

ISOLDE

Dein Schweigen fass' ich, weichst du mir aus.

Weigerst du die Sühne mir?

(Neue Schiffsrufe)

SCHIFFSVLK (von außen)

Ho! he! ha! he!

(Auf Isolde's ungeduldigen Wink reicht Brangäne ibr die gefüllte Trinkschale.)

ISOLDE (mit dem Becher zu Tristan tretend, der ibr starr in die Augen blickt)

Du hörst den Ruf?

Wir sind am Ziel:

in kurzer Frist

(Sehr ernst)

stehn wir -

(Mit leisem Hohne)

vor König Marke.

Geleitest du mich,

dünkt dich's ${ }^{\text {xxxvIII }}$ nicht lieb,

darfst du so ihm sagen?

»Mein Herr und Ohm,

sieh die dir an!

Ein sanft'res Weib

gewännst du nie.

Ihren Angelobten

erschlug ich ihr einst,

sein Haupt sandt' ich ihr heim;

die Wunde, die

seine Wehr mir schuf,

die hat sie hold geheilt;

mein Leben lag

in ihrer Macht,

das schenkte mir

die milde Magd,

und ihres Landes

Schand' und Schmach,

die gab sie mit darein, -

dein Ehgemahl zu sein.

So guter Gaben

holden Dank se io comprendo quel ch'ella tace,

io taccio quel ch'ella non comprende.

ISOLDA

Se il tuo silenzio io comprendo,

tu mi sfuggi.

Rifiuteresti a me la conciliazione?

(Nuove grida dei marinai)

CIURMA (dal di fuori)

Ho! he! ha! he!

(Ad un impaziente cenno di Isolda, Brangania le porge la coppa piena)

ISOLDA (accostandosi con la coppa a Tristano che la fissa negli occhi)

Odi tu il grido?

Siamo giunti alla meta:...

tra poco

(Molto seriamente)

noi saremo...

(Con lieve scherno)

davanti a re Marke.

Se tu mi accompagnerai,

non ti sembrerà caro,

se gli potrai dire:

"Mio signore e zio,

guárdatela:

una più dolce donna

mai avresti ottenuto.

Il suo fidanzato

un giorno le uccisi,

ed il capo di lui le inviai a casa;

la ferita che

la sua arma m'inferse,

ella me l'ha benignamente guarita;

la mia vita fu

in suo potere:...

me la regalò

la mite fanciulla,

e della sua patria

l'onta e il disonore

ella donò per giunta,

per essere tua consorte.

Di così grandi doni

grata riconoscenza

xxxVIII GS: «dich». 
schuf mir ein süßer

Sühne-Trank:

den bot mir ihre Huld,

zu sühnen ${ }^{\text {xxxix }}$ alle Schuld. «

SCHIFFSRUF (von außen)

Auf das Tau!

Anker los! ${ }^{\mathrm{XL}}$

TRISTAN (wild auffahrend)

Los den Anker!

Das Steuer dem Strom!

Den Winden Segel und Mast!

(Er entreißt Isolden ungestüm die Trinkschale)

Wohl kenn' ich Irlands

Königin,

und ihrer Künste

Wunderkraft:

den Balsam nützt' ich,

den sie bot;

den Becher nehm' ich nun,

daß ganz ich heut genese!

Und achte auch

des Sühne-Eids,

den ich zum Dank dir sage. -

Tristan's Ehre -

höchste Treu':

Tristans Elend -

kühnster Trotz.

Trug des Herzens;

Traum der Ahnung:

ew'ger Trauer

einz'ger Trost,

Vergessens güt'ger Trank!

Dich trink' ich sonder Wank.

(Er setzt an und trinkt)

ISOLDE

Betrug auch hier?

Mein die Hälfte!

(Sie entwindet ihm den Becher)

Verräther, ich trink' sie dir! ${ }^{7}$ mi suscitò una dolce

bevanda di riconciliazione, la quale la grazia di lei mi offerse per cancellare ogni colpa».

LA CIURMA (dal di fuori)

Su le corde!

Via l'ancora!

TRISTANO (alzandosi con impeto)

Alzate l'ancora!

Il timone alla corrente!

$\mathrm{Al}$ vento vele ed albero!...

(Strappa a Isolda con impeto la coppa)

Bene io conosco d'Irlanda

la regina

e delle sue arti

la forza magica.

Io profittai del balsamo

ch'ella m'offerse:

la coppa ora prendo,

così che io del tutto oggi guarisca.

Poni mente anche

al giuramento di conciliazione,

che per ringraziamento ti faccio.

Onor di Tristano...

altissima fedeltà:

sofferenza di Tristano...

arditissima sfida.

Frode del cuore;

fantasma del presagio:

d'un lutto eterno

solo sollievo,

benigna bevanda d'oblio!

io ti bevo senza vacillare.

(Accosta [la coppa] alle labbra e beve)

ISOLDA

Inganno anche qui?

A me la metà!

(Gli strappa la coppa)

Traditore! Alla tua salute la bevo!

xxxix GS: «büßen».

XL GS: «ab!».

7 Isolde canta questo verso su una deformazione del gesto iniziale della partitura, il motivo del Dolore (es. $1 x$ ) cantato dai violoncelli: che ora diventa $\mathrm{Lab}_{3}-\mathrm{Fa}_{4}-\mathrm{Mi}_{4}-\mathrm{Mi} b_{4}$, e si chiude su una riorchestrazione in fortissimo del primo 'accordo del Tristan' (es. 1z: $\mathrm{Fa}_{2}-\mathrm{Si}_{2}-\mathrm{Re}_{3}-\mathrm{Hol}_{3}$, sia pure annotato come $\mathrm{Fa}_{2}-\mathrm{Dob}_{2}$ - $\mathrm{Mi}_{3}$ - $\mathrm{Lab}_{3}$ ). Non è una coincidenza: il liquido della coppa proietta Tristan e Isolde dentro la musica del Vorspiel, del quale (dopo le sostanziose allusioni a metà atto: vedi nota 5) ora udiamo una ripresa pressoché integrale - sia pure riorganizzata 
(Sie trinkt. Dann wirft sie die Schale fort. - Beide, von Schauer erfaßt, blicken sich mit höchster Aufregung, doch mit starrer Haltung, unverwandt in die Augen, in deren Ausdruck der Todestrotz bald der Liebesglut weicht. - Zittern ergreift sie. Sie fassen sich krampfhaft an das Herz, - und fübren die Hand wieder an die Stirn. - Dann suchen sie sich wieder mit dem Blicke, senken ibn verwirrt, und beften ihn von Neuem mit steigender Sehnsucht auf einander)

ISOLDE (mit bebender Stimme)

Tristan!

TRISTAN (überströmend)

Isolde!

ISOLDE (an seine Brust sinkend)

Treuloser Holder!

TRISTAN (mit Glut sie umfassend)

Seligste Frau!

(Sie verbleiben in stummer Umarmung. Aus der Ferne vernimmt man Trompeten und Posaunen, von außen auf dem Schiffe den Ruf der)

MÄNNER

Heil! Heil!

König Marke!

König Marke Heil!

BRANGÄNE (die, mit abgewandtem Gesicht, voll Verwirrung und Schauder sich über den Bord gelehnt hatte, wendet sich jetzt dem Anblick des in Liebesumarmung versunkenen Paares zu, und stürzt bänderingend, voll Verzweiflung, in den Vordergrund)

Wehe! Weh!

Unabwendbar

ew'ge Not

für kurzen Tod!

Tör'ger Treue

trugvolles Werk

blüht nun jammernd empor!

(Tristan und Isolde fahren verwirrt aus der Umarmung auf)
(Ella beve. Quindi getta via la coppa. - Ambedue, presi da un brivido, si guardano con suprema esaltazione, ma senza muoversi, fissamente negli occhi; nei quali l'espressione di una sfida alla morte presto cede a quella della fiamma d'amore. - Un tremito li assale. Si portano spasmodicamente la mano al cuore... e la riportano nuovamente alla fronte. - Poi si cercano nuovamente con lo sguardo, lo abbassano smarriti, e quindi nuovamente lo fissano l'uno sull'altra con crescente passione)

ISOLDA (con voce tremante)

Tristano!

TRISTANO (prorompendo) Isolda!

ISOLDA (cadendo nelle sue braccia)

Amico infedele!

TRISTANO (abbracciandola con ardore)

Dolcissima donna!

(Rimangono in muto amplesso. Di lontano si sentono trombe e tromboni; ed all'esterno sul vascello il grido degli)

UOMINI

Salve! Salve!

Re Marke!

Re Marke, salve!

BRANGANIA (che col viso distolto, piena di smarrimento e di orrore, si era appoggiata al parapetto, si volge ora a guardare la coppia sprofondata nell'amoroso amplesso, e si precipita torcendosi le mani per la disperazione verso il davanti della scena)

Guai! Guai!

Ineluttabile

eterno soffrire

invece d'una rapida morte!

Di una folle fedeltà

la frodolenta opera

fiorirà ormai nel dolore!

(Tristano e Isolda si svincolano smarriti dall'abbraccio)

segue nota 7

e tutt'altro che letterale. Ripresa drammaticamente disturbata dal mondo del 'giorno' (le grida degli uomini che salutano re Marke, la lucida disperazione di Brangäne), che preme dall'esterno e reclama i suoi diritti. Dopo questa prima interruzione, il Vorspiel riprende febbrilmente il suo corso, fino a infrangersi - all'apertura delle cortine - sull'eloquente Do della musica del 'giorno': quella della festa rumorosa dei cavalieri e dei marinai, alla quale si uniscono le fanfare di trombe e tromboni dietro le quinte (combinate con una riscrittura del motivo di «Frisch weht der Wind»: es. 6). Per l'ultima volta il Vorspiel riguadagna la scena («Was ist, Brangäne?»), per essere spazzato via dalle ultime battute di esultanza collettiva in Do. 
TRISTAN

Was träumte mir

von Tristans Ehre?

ISOLDE

Was träumte mir

von Isoldes Schmach?

TRISTAN

Du mir verloren?

ISOLDE

Du mich verstoßen?

TRISTAN

Trügenden Zaubers

tückische List!

ISOLDE

Törigen Zürnens

eitles Dräun!

TRISTAN

Isolde!

ISOLDE

Tristan!

TRISTAN

Süßeste Maid!

ISOLDE

Trautester Mann! ${ }^{\mathrm{XLI}}$

BEIDE

Wie sich die Herzen

wogend erheben!

Wie alle Sinne

wonnig erbeben!

Sehnender Minne

schwellendes Blühen,

schmachtender Liebe

seliges Glühen!

Jach in der Brust

jauchzende Lust!

Isolde! Tristan!

Tristan! Isolde!

Welten-entronnen

du mir gewonnen!

Du mir einzig bewußt, höchste Liebes-Lust!
TRISTANO

Che cosa mi sognai

dell'onor di Tristano?

ISOLDA

Che cosa mi sognai

dell'onta di Isolda?

TRISTANO

Tu perduta per me?

ISOLDA

Io da te respinta?

TRISTANO

D'ingannevole magia

perfida astuzia!

ISOLDA

D'una folle ira,

vana minaccia!

TRISTANO

Isolda!

ISOLDA

Tristano!

TRISTANO

Dolcissima fanciulla!

ISOLDA

Dilettissimo uomo!

AMBEDUE

Come i cuori

ondeggiando balzano!

Come tutti i sensi

tremano di voluttà!

D'una bramosa passione

ondeggiante fiorire,

d'un languente amore

ardere beato!

Súbita nel petto

gioia gioiosa!

Isolda! Tristano!

Tristano! Isolda!

Sottratto al mondo,

o tu a me conquistato!

A me solo cognita

altissima gioia d'amore!

xLI GS: «ISOLDE | Trautester Mann! | TRISTAN | Süßeste Maid!». 
(Die Vorbänge werden weit auseinander gerissen. Das ganze Schiff ist von Rittern und Schiffsleuten erfüllt, die jubelnd über Bord winken, dem Ufer zu, das man, mit einer bohen Felsenburg gekrönt, nahe erblickt. Tristan und Isolde bleiben, in ihren gegenseitigen Anblick verloren, ohne Wahrnehmung des um sie Vorgehenden)

BRANGÄNE (zu den Frauen, die auf ibren Wink aus dem Schiffsraum heraufsteigen)

Schnell den Mantel,

den Königsschmuck!

(Zwischen Tristan und Isolde stürzend)

Unsel'ge! Auf!

Hört wo wir sind.

(Sie legt Isolden, die es nicht gewahrt, den Mantel um. Trompeten und Posaunen, vom Lande her, immer deutlicher.)

ALLE MÄNNER
Heil! Heil!
König Marke!
König Marke Heil!
Heil! Heil dem König!
KURWENAL (lebhaft herantretend)
Heil Tristan!
Glücklicher Held! -
ALLE MäNNER
Heil König Marke!
KURWENAL
Mit reichem Hofgesinde
dort auf Nachen
naht Herr Marke.
Hei! wie die Fahrt ihn freut,
daß er die Braut sich freit!
TRISTAN (in Verwirrung aufblickend)
Wer naht?
KURWENAL
TRISTAN Der König.
Welcher König?
(Kurwenal deutet über Bord.)
DIE MÄNNER (die Hüte schwenkend)
Heil! König Marke!
(Tristan starrt wie sinnlos nach dem Lande)
XLII

(I cortinaggi vengono aperti in tutta la loro ampiezza; tutto il vascello è pieno di cavalieri e marinai, che al di sopra del parapetto accennano alla riva, la quale, coronata da un alto castello, si vede vicina. Tristano ed Isolda rimangono perduti nella loro reciproca contemplazione, senza avvedersi di quel che avviene intorno a loro.)

BRANGANIA (alle donne che al suo cenno salgono sopra coperta)

Presto il mantello,

l'ornamento regale!

(Precipitandosi fra Tristano e Isolda)

Infelici! Su!

Udite dove siamo.

(Ella impone ad Isolda, che non se ne avvede, il mantello. Trombe e tromboni, dalla terraferma, sempre più distinti.)

TUTTI GLI UOMINI

Salve! Salve!

Re Marke!

Re Marke, salve!

Salve! Salve al re!

KURWENAL (avanzandosi con vivacità)

Salve Tristano!

Fortunato Eroe!

TUTTI GLI UOMINI

Re Marke, salve!

KURWENAL

Con ricco seguito

colà sulla barca

s'appressa re Marke.

Evviva! Come lo allieta il tragitto

per congiungersi alla fidanzata!

TRISTANO (levando smarrito lo sguardo)

Chi s'appressa?

KURWENAL

Il re.

TRISTANO

Quale re?

(Kurwenal accenna oltre il parapetto.)

GLI UOMINI (agitando il berretto)

Salve! Re Marke!

(Tristano guarda verso la terra con gli occhi fissi, come fuori di coscienza)

XLII GS: «TRISTAN | Marke! Was will er? I (Er starrt wie sinnlos nach dem Lande)». 
ISOLDE (in Verwirrung, zu Brangäne)

Was ist, Brangäne?

Welcher Ruf? ${ }^{\mathrm{XIIII}}$

BRANGÄNE

Isolde! Herrin!

Fassung nur heut!

ISOLDE

Wo bin ich? Leb' ich?

Ha, welcher Trank?

BRANGÄNE (verzweiflungsvoll)

Der Liebestrank.

ISOLDE (starrt entsetzt auf Tristan)

Tristan!

TRISTAN

Isolde!

ISOLDE

Muß ich leben?

(Sie stürzt obnmächtig an seine Brust.)

BRANGÄNE (zu den Frauen)

Helft der Herrin!

TRISTAN

O Wonne voller Tücke!

O Trug-geweihtes Glücke!

(Ausbruch allgemeinen Jauchzens)

DIE MÄNNER

Kornwall Heil! ${ }^{\mathrm{XLIV}}$

(Leute sind über Bord gestiegen, andere haben eine Brücke ausgelegt, und die Haltung aller deutet auf die soeben bevorstehende Ankunft der Erwarteten, als der Vorhang schnell fällt.)
ISOLDA (SMARRITA, a Brangania)

Che c'è Brangania?

Quale grido?

BRANGANIA

Isolda! Signora!

Sii in te, oggi almeno!

ISOLDA

Dove mi trovo? Vivo?

Ah! quale filtro?

BRANGANIA (disperatamente)

Il filtro d'amore.

ISOLDA (fissa atterrita Tristano)

Tristano!

TRISTANO

Isolda!

ISOLDA

Devo io vivere?

(Cade svenuta nelle sue braccia)

BRANGANIA (alle donne)

Soccorrete alla signora!

TRISTANO

$\mathrm{O}$ voluttà piena di frode!

O felicità consacrata dall'inganno!

(Esplosione di giubilo generale)

GLI UOMINI

Cornovaglia salve!

(Della gente è salita scavalcando $i$ parapetti, altri hanno collocato un ponte, ed il contegno di tutti accenna all'imminente arrivo degli attesi, quando il sipario cala rapidamente.)

xLIII GS: «Was ist? Brangäne! | Ha! Welcher Ruf?».

xLIV GS: «DIE MÄNNER | Heil dem König! | Kornwall Heil!». 


\section{ZWEITER AUFZUG}

\section{ERSTE SZENE ${ }^{8}$}

Garten mit hohen Bäumen vor dem Gemache Isoldes, zu welchem, seitwärts gelegen, Stufen hinaufführen. Helle, anmuthige Sommernacht. An der geöffneten Türe ist eine brennende Fackel aufgesteckt.

\section{SCENA PRIMA}

Giardino con alti alberi davanti alla camera d'Isolda, alla quale conducono, di fianco, alcuni gradini. Chiara, deliziosa notte d'estate. Presso la porta aperta è confitta una torcia accesa.

8 L'«introduzione» orchestrale (Einleitung) dell'atto secondo si oppone a quella del primo per il piglio sehr lebhaft («molto vivace») del tempo, e per la dinamica fortissimo delle battute d'esordio: e tuttavia, anche qui il primo accordo è carico di tensione (Mib-Sol-Re) e risolve su un accordo a sua volta dissonante, dunque bisognoso di risoluzione (la settima diminuita Mit-Sol-Sib-Reb). È il consueto principio tristaniano: «un desiderio senza meta perché ogni meta è solo un nuovo desiderio». Del resto, l'atto si apre all'insegna della continuità con l'ultima scena del primo: nulla è cambiato da quando, sul ponte del vascello di Tristan, si è messo in moto il «desiderio inestinguibile» degli amanti. In questo incipit flauti, oboi e violini incidono il motivo del Giorno (Tagesmotiv): ESEMPIO 11 (II, bb. 1-3)

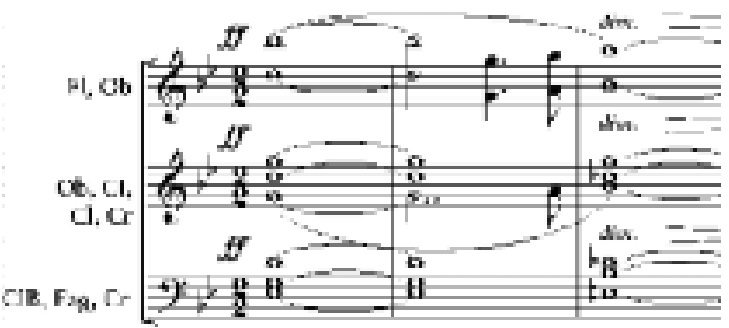

Sul tremolo degli archi, la dinamica collassa immediatamente verso il piano, e in poche battute ci troviamo in attesa sulla dominante della tonalità che governa l'introduzione e la prima scena nel suo complesso, Sib. Ad animare la sosta è il profilo ascendente del motivo dell'Impazienza (Thema der Ungeduld), nel piano del clarinetto basso, sotto le figure febbrili degli archi divisi:

ESEMPIO 12 (bb. 9-10)

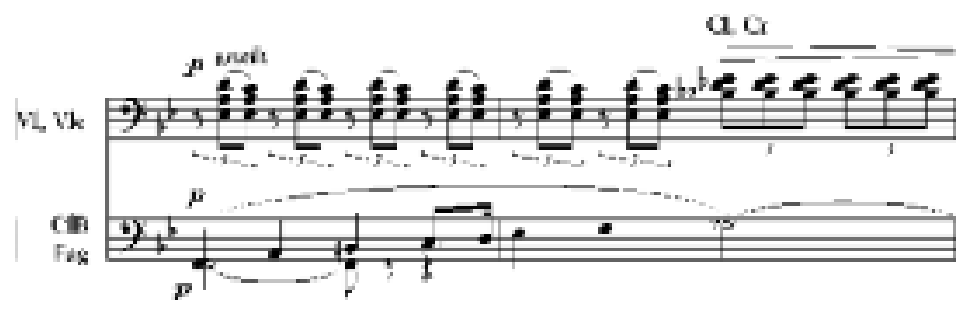

Ma l'introduzione ha in serbo un altro paio di idee musicali che saranno decisive per il resto del dramma. La prima si combina senza intralciarlo con l'incedere costante dell'es. 12, all'altra estremità dello spettro sonoro, nel timbro del flauto (motivo del Richiamo d'amore: Liebesrufmotiv):

ESEMPIO 13 (bb. 29-32)

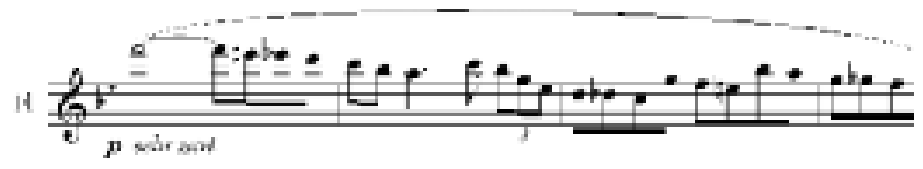


(Jagdgetön. Brangäne, auf den Stufen am Gemache, späbt dem immer entfernter vernebmbaren Jagdtrosse nach. Brangäne blickt ängstlich in das Gemach zurück, darin sie Isolde nahen sieht. Isolde tritt, feurig bewegt, aus dem Gemach zu Brangäne')

ISOLDE

Hörst du sie noch?

Mir schwand schon fern der Klang.

BRANGÄNE (lauschend)

Noch sind sie nah:

deutlich tönt's da her.

ISOLDE (lauschend)

Sorgende Furcht

beirrt dein $\mathrm{Ohr}$;

dich täuscht des Laubes

säuselnd Getön',

das lachend schüttelt der Wind.

BRANGÄNE

Dich täuscht des ${ }^{\text {II }}$ Wunsches

Ungestüm,
(Fanfare di caccia. Brangania, sui gradini che conducono alla camera, presta ascolto al tumulto della caccia che sempre più s'allontana. Guarda impensierita dietro di sé nella camera e vi scorge Isolda che si approssima. Uscendo dalla camera, Isolda viene verso Brangania, in grande agitazione.)

ISOLDA

Li odi tu ancora?

Già a me sparve lontano il clamore.

BRANGANIA (prestando orecchio)

Ancora sono vicini;...

da questa parte si sente chiaro il rumore.

ISOLDA (prestando ascolto)

Un timore angoscioso

inganna il tuo orecchio.

T'illude del fogliame

la sussurrante canzone,

cui ridendo agita il vento.

BRANGANIA

Te illude del desiderio

l'impeto,

segue nota 8

La seconda, presentata dai violini in fortissimo (motivo della Beatitudine: Seligkeitsmotiv), è ben altrimenti carica di passionalità e compare all'apice di un molto crescendo innescato dal motivo del Desiderio (es. 1y):

ESEMPIO 14 (bb. 45-46)

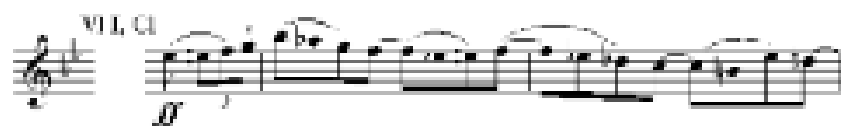

Al levarsi del sipario, infine, dalle quinte giunge ovattato il suono della fanfara di sei corni (sfruttati da Wagner nel loro potenziale di Jagdhörner, di strumenti da caccia), che scompare lentamente con un effetto di dissolvenza orchestrato con precisione dal compositore. Il resto della scena indugia su tutti questi materiali tematici, che dipingono l'ascolto inquieto, l'agitazione, il desiderio di Isolde. È un magistrale quadro notturno pieno di ombre, sussurri (sordine, suoni chiusi dei corni e sul ponticello degli archi) e accensioni improvvise, con il quale Wagner ha dischiuso non poche vie alla modernità. Un ulteriore, importante elemento tematico viene dall'assolo di Isolde su Monna Minne - ovvero «Dama Passione», come traduce letteralmente Franco Serpa («Frau Minne kenntest du nicht?»). Il coronamento di questa pagina è infatti un tema d'Amore in La (Liebesthema: es. 15), imparentato con il Richiamo d'amore (es. 13) e cantato dai violini in ottave, sul quale Isolde si dichiara posseduta dalla Dama ("wie sie es wendet» ecc.):

ESEMPIO 15 (bb. 419-420)

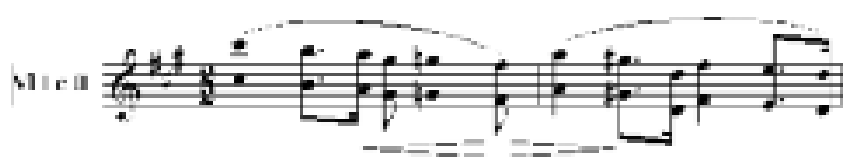

I Gs: «Zu ihr tritt aus dem Gemache, feurig bewegt, Isolde».

II GS: «deines». 
zu vernehmen was du wähnst: -

(Sie lauscht)

ich höre der Hörner Schall.

ISOLDE (wieder lauschend)

Nicht Hörnerschall

tönt so hold;

des Quelles sanft

rieselnde Welle

rauscht so wonnig da her:

wie hört' ich sie,

tosten noch Hörner?

Im schweigender ${ }^{\text {III }} \mathrm{Nacht}$

nur lacht mir der Quell:

der meiner harrt

in schweigender Nacht,

als ob Hörner noch nah dir schallten,

willst du ihn fern mir halten?

BRANGÄNE

Der deiner harrt -

o hör mein Flehen! ${ }^{\text {IV }}$

dess' harren Späher zur Nacht.

Weil du erblindet, wähnst du den Blick

der Welt erblödet für euch? -

Als $^{\mathrm{v}}$ dort an Schiffes Bord,

von Tristans bebender Hand

die bleiche Braut

kaum ihrer mächtig,

König Marke empfing, -

als alles verwirrt

auf die Wankende sah,

der güt'ge König,

mild besorgt,

die Mühen der langen Fahrt,

die du littest, laut beklagt':

ein Einz'ger war's -

ich achtet' es wohl -

der nur Tristan faßt' in's Auge;

mit böslicher List,

lauerndem Blick

sucht' er in seiner Miene

zu finden, was ihm diene. di udire, quel che tu pensi.

(Presta ascolto)

Io odo il suono dei corni.

ISOLDA (prestando nuovamente ascolto)

Non suono di corni

così gratamente suona:

della fonte la dolcemente

mormorante onda

mormora verso di noi così soavemente.

Come l'udrei,

se rimbombassero i corni ancora?

Nel silenzio della notte,

solo mi ride la fonte;

colui che m'attende

nella silente notte,

come se i corni ancora vicino a te suonassero, vuoi tu tenermelo lontano?

BRANGANIA

Colui che t'attende, -

O odi il mio avviso! -

Lui attendono spie durante la notte.

Perché tu sei cieca,

credi tu che la vista

del mondo sia indebolita per voi?

Quando colà, a bordo del vascello,

dalla tremante mano di Tristano

la pallida fidanzata, appena in grado di reggersi,

il re Marke accolse;

quando tutti smarriti

guardarono la vacillante,

il buon re,

con dolce premura,

la fatica del lungo viaggio,

che tu avevi sopportato, ad alta voce compianse:...

un solo fu,

io ben l'osservai,

che solo Tristano tenne d'occhio;

con maligna astuzia,

con sguardo indagatore,

egli cercava nel suo viso

di trovare quel che a lui servisse.

\footnotetext{
III GS: «Schweigen der».

IV GS: «Warnen!».

v GS: «Da».
} 
Tückisch lauschend

treff' ich ihn oft:

der heimlich euch umgarnt,

vor Melot seid gewarnt.

ISOLDE

Meinst du Herrn Melot?

$\mathrm{O}$ wie du dich trügst!

Ist er nicht Tristans

treuester Freund?

Muß mein Trauter mich meiden,

Dann weilt er bei Melot allein.

BRANGÄNE

Was mir ihn verdächtig,

macht dir ihn teuer.

Von Tristan zu Marke

ist Melots Weg;

dort sät er üble Saat.

Die heut im Rat

dies nächtliche Jagen

so eilig schnell beschlossen, einem edlern Wild, als dein Wähnen meint, gilt ihre Jägers-List.

ISOLDE

Dem Freunde zu Lieb'vI

erfand diese List

aus Mit-Leid

Melot der Freund:

nun willst du den Treuen schelten?

Besser als du

sorgt er für mich;

ihm öffnet er,

was mir du ${ }^{\text {vII }}$ sperrst.

O spare mir des Zögerns Not!

Das Zeichen, Brangäne!

o gieb das Zeichen!

Lösche des Lichtes

letzten Schein!

$\mathrm{Daß}$ ganz sie sich neige,

winke der Nacht!

Schon goß sie ihr Schweigen

durch Hain und Haus;
Perfidamente in ascolto,

spesso io l'incontro:...

costui vi tende segretamente una rete:

guardatevi da Melot.

ISOLDA

Intendi tu di sire Melot?

oh, come t'inganni!

Non è egli di Tristano

l'amico più fido?

Quando il mio amatore deve evitarmi, solo con Melot egli s'indugia.

BRANGANIA

Quel che me lo rende sospetto,

lo rende caro a te!

Da Tristano a Marke

va la strada di Melot;...

colà cattiva semenza ei semina.

Coloro che oggi in consiglio

questa caccia notturna

così precipitosamente decisero...

a più nobile selvaggina,

che la tua mente pensi,

vale la loro astuzia di cacciatori.

ISOLDA

Per amor dell'amico

trovò quest'astuzia,

per compassione,

Melot, l'amico.

Vuoi tu ora accusare quel fido?

Meglio di te

egli a me provvede;

all'amico egli apre

le vie che tu mi chiudi.

O risparmiami l'angoscia dell'attendere!

Il segnale, Brangania!

Oh! da' il segnale!

Spegni della luce

l'ultimo barbaglio!

Perché tutta s'inchini, accenna alla notte!

Già ella versò il suo silenzio

per le selve e le case,

VI GS: «lieb».

vII GS: «du mir». 
schon füllt sie das Herz

mit wonnigem Graus:

o lösche das Licht nun aus!

Lösche den scheuchenden Schein!

Laß meinen Liebsten ein!

BRANGÄNE

O lass' die warnende Zünde!

Laß die Gefahr ${ }^{\mathrm{VIII}}$ sie dir zeigen! -

O wehe! Wehe!

Ach mir Armen!

Des unseligen Trankes!

Daß ich untreu

einmal nur

der Herrin Willen trog!

Gehorcht' ich taub und blind,

dein - Werk

war dann der Tod:

doch deine Schmach,

deine schmählichste Not,

mein - Werk

muß ich Schuld'ge es ${ }^{\mathrm{IX}}$ wissen!

ISOLDE

Dein - Werk?

O tör'ge Magd!

Frau Minne kenntest du nicht?

Nicht ihres Zaubers ${ }^{\mathrm{x}}$ Macht?

Des kühnsten Mutes

Königin,

des Welten-Werdens

Walterin,

Leben und Tod

sind untertan ihr, ${ }^{\mathrm{XI}}$

die sie webt aus Lust und Leid, in Liebe wandelnd den Neid.

Des Todes Werk, nahm ich's vermessen zur Hand,

Frau Minne hat

es meiner Macht ${ }^{\mathrm{XII}}$ entwandt.

Die Todgeweihte

\footnotetext{
VIII GS: «Die Gefahr laß».

IX GS: «sie».

x GS: «ihrer Wunder».

XI GS: «ihr untertan,».

XII GS: «meiner Macht es».
}

già ella riempie i cuori

d'un orrore voluttuoso.

Oh! spegni, dunque, la luce!

Spegni il bagliore che allontana!

$\mathrm{Fa}$ ' che il mio adorato entri!

BRANGANIA

Oh lascia la fiamma che ti guarda, lascia che t'insegni il pericolo!

O guai! guai!

Povera me!

Sciagurato filtro!

Che infedele

una sol volta,

ingannai la volontà della mia signora!

Avessi obbedito sorda e cieca,

opera tua

sarebbe stata la morte.

Invece il tuo disonore,

la tua miseranda angoscia,...

opera mia

debbo io colpevole riconoscere!

ISOLDA

Opera... tua?

O fanciulla semplicetta!

Monna Minne non conosci?

Non la potenza dei suoi miracoli?

Del più temerario coraggio

regina?

Del divenire del mondo

reggitrice?

Vita e morte

sono a lei soggetti;

ella li intesse di piacere e di patire, in passione mutando l'invidia.

L'opera della morte

l'avevo temerariamente intrapresa,...

Monna Minne l'ha

sottratta al mio potere.

La consacrata alla morte 
nahm sie in Pfand,

faßte das Werk

in ihre Hand;

wie sie es wendet,

wie sie es endet,

was sie mir kühre,

wohin mich führe, ${ }^{\mathrm{XIV}}$

ihr ward ich zu eigen: -

nun laß mich gehorsam zeigen!

BRANGÄNE

Und mußte der Minne

tückischer Trank

des Sinnes Licht dir verlöschen;

darfst du nicht sehen,

wenn ich dich warne:

nur heute hör,

o hör mein Flehen!

Der Gefahr leuchtendes Licht -

nur heute! heut! -

die Fackel dort lösche nicht!

ISOLDE ${ }^{\mathrm{XV}}$

Die im Busen mir

die Glut entfacht,

die mir das Herze

brennen macht,

die mir als Tag

der Seele lacht,

Frau Minne will,

es werde Nacht,

daß hell sie dorten leuchte,

(während sie auf die Fackel zueilt)

wo sie dein Licht verscheuchte. -

(Sie nimmt die Fackel von der Türe)

Zur Warte du!

Dort wache treu.

Die Leuchte -

wär's meines Lebens Licht, -

lachend

sie zu löschen zag' ich nicht.

(Sie wirft die Fackel zur Erde, wo sie allmählich verlischt. ${ }^{\mathrm{XVI}}$ Brangäne wendet sich bestürzt ab, um auf come pegno ella ha preso,

e l'opera assunta

nelle sue mani.

Comunque la volga,

comunque la compia,

quel ch'ella mi scelga,

ovunque mi conduca,

sua serva sono diventata:

lascia dunque ch'io mi mostri obbediente!

BRANGANIA

Se dovesse dell'amore

il frodolento filtro

la luce spegnerti dello spirito;

se non puoi vedere

quello ond'io ti guardo:

almeno oggi odi, oh odi il mio supplicare!

Del pericolo la luce lucente solo oggi, oggi!

la fiaccola colà non spegnere!

ISOLDA

Colei che in cuore a me

venta la fiamma,

che a me il cuore

fa ardere,

colei che a me qual giorno

ride alla mia anima...

Monna Minne vuole:

si faccia notte,

perché ella chiara risplenda colà,

(si affretta alla fiaccola)

dove la tua luce l'ha cacciata.

(Prende la fiaccola dalla porta)

Tu in vedetta:

colà veglia fedele!

La fiaccola,

fosse anche la luce della mia vita,...

ridendo

non tremo di spegnere!

(Ella getta la fiaccola a terra, dove a poco a poco si spenge. Brangania si ritrae costernata, per raggiun-

\footnotetext{
XIII GS: «kühret,».

XIV GS: «führet».

XV GS: «ISOLDE I (auf die Fackel zueilend und sie erfassend)».

${ }^{\mathrm{xVI}}$ GS: «Sie hat die Fackel herabgenommen und verlöscht sie am Boden».
} 
einer äußeren Treppe die Zinne zu ersteigen, wo sie langsam verschwindet. Isolde lauscht und späht, zunächst schüchtern, in einen Baumgang. Von wachsendem Verlangen bewegt, schreitet sie dem Baumgang näher und späht zuversichtlicher. Sie winkt mit einem Tuche, erst seltener, dann häufiger, und endlich, in leidenschaftlicher Ungeduld, immer schneller. Eine Gebärde des plötzlichen Entzückens sagt, daß sie den Freund in der Ferne gewahr geworden. Sie streckt sich höher und höher, und, um besser den Raum zu übersehen, eilt sie zur Treppe zurück, von deren oberster Stufe aus sie dem Herannahenden zuwinkt. ${ }^{\mathrm{xVII}}$ )

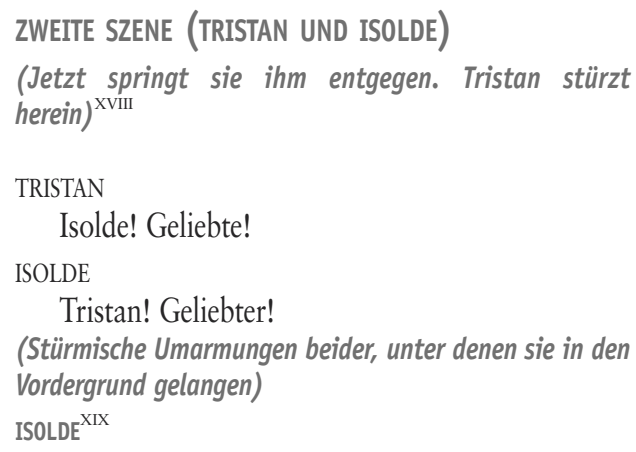

Bist du mein??

TRISTAN

Hab' ich dich wieder?

ISOLDE

Darf ich dich fassen? gere, salendo una scala esterna, la terrazza merlata, sulla quale essa lentamente scompare. (Isolda orecchia e spia, dapprima timidamente, in un viale. Mossa da crescente desiderio, s'accosta al viale e spia con maggior sicurezza. Ella accenna con un fazzoletto, dapprima a rari intervalli, poi più spesso, ed infine con appassionata impazienza, sempre più rapidamente. Un gesto di gioia improvvisa dice che ha scorto l'amico in lontananza. Ella si drizza sempre più, sempre più, e per dominare meglio il terreno, retrocede in fretta alla scala, dal gradino piu alto della quale, ella accenna all'amico che s'avvicina.)

\section{SCENA SECONDA (TRISTANO E ISOLDA)}

(A questo punto ella gli balza incontro. Tristano entra precipitosamente)

TRISTANO

Isolda! Cara!

ISOLDA

Tristano! Caro!

(Impetuosi amplessi, durante i quali essi raggiungono il proscenio.)

ISOLDA

Sei tu mio?

TRISTANO

Ti ho nuovamente?

ISOLDA

Ti posso abbracciare?

xVII GS: «Isolde blickt erwartungsvoll in einen Baumgang. Sie winkt. Ihre entzückte Gebärde deutet an, daß sie den von fern herannahenden Freund gewahr geworden. Ungeduldige höchste Spannung. -».

xVIII GS: «Tristan stürzt herein; sie fliegt ihm mit einem Freudenschrei entgegen. Glühende Umarmung».

xIx GS: l'intera sequenza di versi fino a «Ewig, ewig ein!» è attribuita a entrambi i personaggi; si è provveduto a segnalare il cambio di personaggio in base alla partitura.

9 È il momento, per dirla con Wagner, delle «effusioni dei sentimenti a lungo repressi» e delle «alte esclamazioni di gioia» (dall'abbozzo in prosa del 1857). Dall'istante in cui Isolde ha scorto Tristan in lontananza, l'orchestra ha cominciato a ripetere in modo ossessivo un disegno (es. 16) che - con l'abbraccio della coppia - ha ceduto il posto a un frenetico motivo della Beatitudine (es. 14). Ora, durante i rapidi scambi dei due amanti, l'es. 16 riprende quota, si fa canto ("O süße, hehrste») e dialoga con il tema d'Amore (es. 15). Il tutto, mentre Tristan e Isolde celebrano ripetutamente l'essere-uno, la soppressione della distanza, con l'unisono delle loro voci. L'es. 16 e il tema d'Amore continuano poi a impegnare l'orchestra mentre gli amanti rievocano la desolazione della lontananza («Wie lange fern!»); ai due motivi si aggiunge qui il profilo affine del Richiamo d'amore (es. 13). ESEMPIO 16 (bb. 531-532)

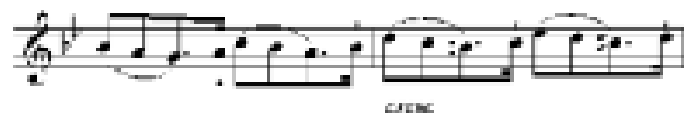


TRISTAN

Kann ich mir trauen?

ISOLDE

Endlich! Endlich!

TRISTAN

An meiner Brust!

ISOLDE

Fühl' ich dich wirklich?

TRISTAN

Seh' ich dich selber? ${ }^{\mathrm{xx}}$

ISOLDE

Dies deine Augen?

TRISTAN

Dies dein Mund?

ISOLDE

Hier deine Hand?

TRISTAN

Hier dein Herz?

ISOLDE

Bin ich's? Bist du's?

Halt' ich dich fest?

TRISTAN

Bin ich's? Bist du's?

Ist es kein Trug?

BEIDE

Ist es kein Traum?

O Wonne der Seele!

O süße, hehrste,

kühnste, schönste,

seligste Lust!

TRISTAN

Ohne Gleiche!

ISOLDE

Überreiche!

TRISTAN

Überselig!

ISOLDE

Ewig!

TRISTAN

Ewig!
TRISTANO

Posso credere a me stesso?

ISOLDA

Finalmente! Finalmente!

TRISTANO

Al mio cuore!

ISOLDA

Veramente ti sento?

TRISTANO

Proprio te vedo?

ISOLDA

Sono questi i tuoi occhi?

TRISTANO

Questa la tua bocca?

ISOLDA

Qui la tua mano?

TRISTANO

Qui il tuo cuore?

ISOLDA

Sono proprio io? Sei proprio tu?

Ti tengo stretto?

TRISTANO

Sono proprio io? Sei proprio tu?

Non è un inganno?

AMBEDUE

Non è un sogno?

O delizia dell'anima,

o dolce, nobilissima,

arditissima, bellissima,

beatissima gioia!

TRISTANO

Senza pari!

ISOLDA

Traboccante!

TRISTANO

Sovrumana!

ISOLDA

Eterna!

TRISTANO

Eterna!

xx GS: «Bist du es selbst?». 


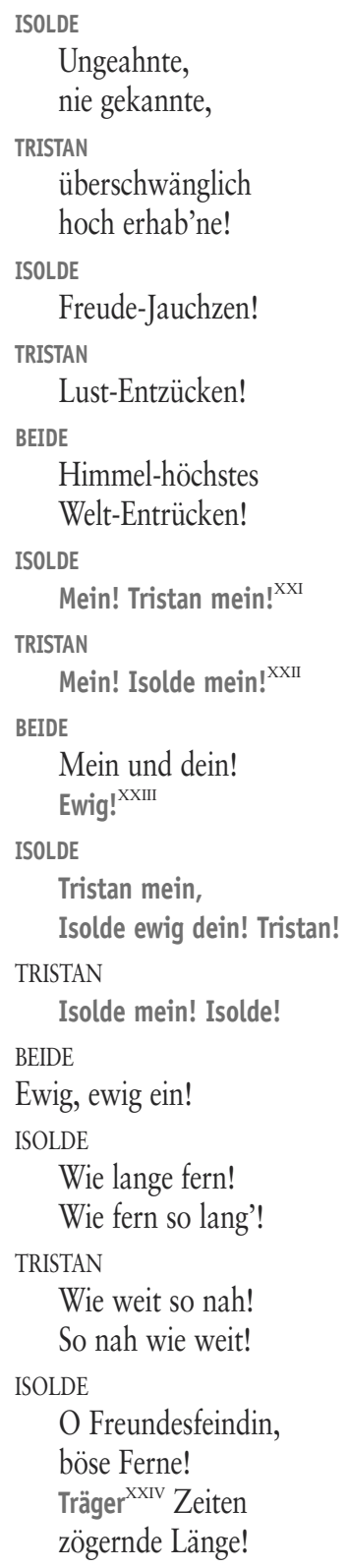

Tristan mein,

Isolde ewig dein! Tristan!

TRISTAN

Isolde mein! Isolde!

BEIDE

Ewig, ewig ein!

ISOLDE

Wie lange fern!

Wie fern so lang'!

TRISTAN

Wie weit so nah!

So nah wie weit!

ISOLDE

O Freundesfeindin,

böse Ferne!

Träger $^{\text {XxIv }}$ Zeiten

zögernde Länge!

ISOLDA

Non presentita,

mai conosciuta,

TRISTANO

sconfinata, alta, sublime!

ISOLDA

Ebrezza di gioia!

TRISTANO

Estasi di piacere!

AMBEDUE

Altissimo celeste

rapimento dal mondo!

ISOLDA

Mio! Tristano mio!

TRISTANO

Mia! Isotta mia!

AMBEDUE

Mio e tuo!

Eternamente!

ISOLDA

Tristano mio,

Isolda eternamente tua! Tristano!

TRISTANO

Isolda mia! Isolda!

AMBEDUE

Eternamente, eternamente uno!

ISOLDA

Quanto tempo lontani!

Come lontani per tanto tempo!

TRISTANO

Così lontani [essendo] così vicini!

Così vicini [eppure] così lontani!

ISOLDA

O nemica degli amici

maligna lontananza!

Di pigri tempi

indugiante lentezza!

\footnotetext{
xxI GS: «Mein Tristan!».

xxII GS: «Mein Isolde!».

XxIII GS: «Immer ein!».

xxIv GS: «O träger».
} 
TRISTAN

O Weit' und Nähe,

hart entzweite!

Holde Nähe,

öde Weite!

ISOLDE

Im Dunkel du, im Lichte ich!

TRISTAN

Das Licht! Das Licht!

O dieses Licht!

Wie lang verlosch es nicht!

Die Sonne sank,

der Tag verging;

doch seinen Neid

erstickt' er nicht:

sein scheuchend Zeichen

zündet er an,

und steckt's an der Liebsten Türe,

daß nicht ich zu ihr führe.

ISOLDE

Doch der Liebsten Hand

löschte das Licht.

Wess' die Magd sich wehrte,

scheut' ich mich nicht;

in Frau Minnes Macht und Schutz

bot ich dem Tage Trutz.

TRISTAN $^{10}$

Dem Tage! Dem Tage! ${ }^{\mathrm{Xxv}}$

Dem tückischen Tage,

dem härtesten Feinde

Haß und Klage!

Wie du das Licht,

o könnt' ich die Leuchte,

der Liebe Leiden zu rächen,

dem frechen Tage verlöschen!

\section{TRISTANO}

O lontananza e vicinanza, duramente separate!

Grata vicinanza, desolata lontananza!

ISOLDA

Tu all'oscuro, io alla luce!

TRISTANO

La luce! La luce!

O questa luce,

per quanto tempo non si è spenta!

Tramontato era il sole

e scomparso il giorno;

pure la propria invidia

egli non soffocava:

il suo segnale che allontana

egli accende;

e lo infigge presso la porta dell'amata,

perché io non possa recarmi da lei.

ISOLDA

Ma la mano dell'amica

ha spento la luce;

di quello che l'ancella non osò

non ebbi io paura:

nella potenza e nella protezione di monna Minne

io lanciai al giorno la mia sfida.

TRISTANO

$\mathrm{Al}$ giorno! Al giorno!

Al frodolento giorno,

al più crudele dei nemici

odio ed accusa!

Come tu la luce,

oh potess'io la fiaccola,

per vendicare i dolori dell'amore,

al prepotente giorno spengere!

10 Inizia qui il cosiddetto Tagesgespräch, la 'conversazione sul giorno' nella quale indugiano i due amanti: conversazione davvero lunga (al punto che Wagner ne meditò un accorciamento: vedi nota XXXV) e che musicalmente rappresenta la prima vera cesura dalla riunione di Tristan e Isolde in avanti. Ed è una sorta di 'festa orgiastica' (Lorenz) del motivo del Giorno (es. 11), che proprio in queste prime battute fa il suo drammatico reingresso. Come 'tema generatore' di queste pagine (nel solito senso di Hubert), il motivo del Giorno verrà infatti piegato infaticabilmente dalla mano di Wagner a inflessioni le più diverse, che interessano ritmo, intervalli, dinamica, timbro, armonia, intonazione espressiva. Un virtuosismo manipolatorio che ha probabilmente pochi termini di confronto in tutto il Tristan - e non solo.

xxv Gs: «Dem Tag! Dem Tag!». 
Gibt's eine Not, gibt's eine Pein, die er nicht weckt mit seinem Schein? Selbst in der Nacht dämmernder Pracht hegt ihn Liebchen am Haus, streckt mir drohend ihn aus.

ISOLDE

Hegt' ihn die Liebste am eignen Haus, im eignen Herzen hell und kraus hegt' ihn trotzig einst mein Trauter, Tristan, der mich verriet! ${ }^{\mathrm{xxv}}$ War's nicht der Tag, der aus ihm log, als er nach Irland werbend zog, für Marke mich zu frein, dem Tod die Treue zu weihn? TRISTAN

Der Tag! Der Tag, der dich umgliß, dahin, wo sie der Sonne glich,

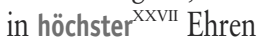
Glanz und Licht Isolden ${ }^{\text {XxVIII }}$ mir entrückt'!

Was mir das Auge so entzückt', das ${ }^{\text {xxix }}$ Herze tief zur Erde drückt': in lichten Tages Schein, wie war Isolde mein?

ISOLDE

War sie nicht dein, die dich erkor, was log der böse
C’è un'angoscia, c'è una pena, ch'egli non susciti con la sua luce?

Anche della notte nella magnificenza crepuscolare, la mia piccola lo mantiene nella sua casa, lo protende minacciosamente contro di me! ISOLDA

Se la tua amica lo mantiene nella propria dimora, nel suo cuore, chiaro, fiammeggiante lo mantenne spavaldamente un giorno il mio amico:

Tristano,... che mi tradi!! Non fu il giorno che in lui menti, quand'egli in Irlanda andò quale messo di nozze, per sposarmi a re Marke, e la sua fedele consacrare alla morte?

TRISTANO

Il giorno! Il giorno

che intorno t'irraggiò, e colà, dove ella rassomigliò al sole, di altissimi onori nella luce e nello splendore, Isolda a me rapi!

Quel che a me l'occhio così estasiava, il cuore profondamente a terra abbatteva: nella chiara luce del giorno, come poteva Isolda essere mia? ISOLDA

Non fu ella tua colei che ti scelse, quale menzogna il maligno

\footnotetext{
XxVI GS: «betrog.».

xxVII GS: "hehrster».

xxvIII GS: «Isolde».

xxIx GS: «mein».
} 
Tag dir vor,

daß, die für dich beschieden, die Traute du verrietest?

TRISTAN

Was dich umgliß

mit hehrster ${ }^{\mathrm{xx}}$ Pracht,

der Ehre Glanz,

des Ruhmes Macht,

an sie mein Herz zu hangen,

hielt mich der Wahn gefangen.

Die mit des Schimmers

hellstem Schein

mir Haupt und Scheitel

licht beschien,

der Welten-Ehren

Tages-Sonne,

mit ihrer Strahlen

eitler Wonne,

durch Haupt und Scheitel

drang mir ein,

bis in des Herzens

tiefsten Schrein.

Was dort in keuscher Nacht

dunkel verschlossen wacht', was ohne Wiss' und Wahn ich dämmernd dort empfah'n, ein Bild, das meine Augen zu sehn ${ }^{\mathrm{XxX}}$ sich nicht getrauten, von des Tages Schein betroffen lag mir's da schimmernd offen.

Was mir so rühmlich

schien und hehr,

das rühmt' ich hell

vor allem Heer:

vor allem Volke

pries ich laut

der Erde schönste

Königin. ${ }^{\mathrm{xx} X I}$

Dem Neid, den mir

der Tag erweckt', ${ }^{\prime}$ xxIII

dem Eifer, den giorno ti mentì, perché, colei che era a te destinata, la tua amata, tu avessi a tradire?

TRISTANO

Come intorno ti irraggiarono

con nobile magnificenza

lo splendore dell'onore,

e la potenza della gloria;

di stringermi ad essi col mio cuore,

m'irretì il mio delirio.

La stella, che del suo splendore

con la più lucente fiamma,

a me capo e fronte

chiaramente illuminava -

degli onori mondani

il sole del giorno -

dei suoi raggi

con la vana delizia

attraversando e capo e fronte,

penetrò in me

del cuore

fin nella più profonda lacuna.

Quel che, colà in casta notte

chiuso all'oscuro vegliava,

quel che, senza saperlo e senza sognarlo

in quella lacuna crepuscolare avevo accolto:

un'immagine, che i miei occhi

non si fidavano di contemplare,

colpita dai raggi del giorno

mi apparve aperta nel suo splendore.

Quel che a me così glorioso

appariva ed augusto,

io lo vantai apertamente

davanti a tutte le schiere;

davanti a tutto il popolo

ad alta voce celebrai

come la più bella della terra,

la regina.

All'invidia, che contro di me

il giorno aveva suscitato,

alla gelosia, che

\footnotetext{
xxx GS: «hehrer».

XxxI GS: «schaun».

xxxII GS: «Königs-Braut».

xxxIII GS: «erweckt».
} 
mein Glücke schreckt', der Mißgunst, die mir Ehren

und Ruhm begann zu schweren, denen bot ich Trotz, und treu beschloß, um Ehr' und Ruhm zu wahren, nach Irland ich zu fahren.

ISOLDE

O eitler Tages-Knecht! Getäuscht von ihm, der dich getäuscht, wie mußt' ich liebend um dich leiden, den, in des Tages falschem Prangen, von seines Gleißens Trug befangen, ${ }^{\text {xxxIV }}$ dort, wo ihn Liebe heiß umfaßte, im tiefsten Herzen hell ich haßte! Ach, in des Herzens Grunde wie schmerzte tief die Wunde! Den dort ich heimlich barg, wie dünkt' er mich so arg wenn in des Tages Scheine der treu gehegte Eine der Liebe Blicken schwand, als Feind nur vor mir stand.

Das als Verräter dich mir wies, dem Licht des Tages wollt' ich entfliehn, dorthin in die Nacht dich mit mir ziehn, wo der Täuschung Ende mein Herz mir verhieß, wo des Trugs geahnter

Wahn zerrinne: dort dir zu trinken ew'ge Minne, mit mir - dich im Verein wollt' ich dem Tode weihn. la mia fortuna aveva atterrito, al disfavore, che al mio onore ed alla mia fama cominciava a portar danno: a tutti io lanciai la mia sfida, e fedelmente decisi, per conservare il mio onore e la mia gloria, di recarmi in Irlanda.

ISOLDA

O vano servo del giorno!

Da quello illuso

che t'illudeva, quanto dovetti io amando

per te soffrire!

Colui, del giorno

nella falsa magnificenza,

e del suo splendere

dall'inganno preso, colà, dove l'amore ardentemente l'aveva accolto, dal più profondo del cuore apertamente odiai.

Ah! nel profondo del cuore, come profondo mi straziava la ferita!

Colui che colà segretamente avevo celato, come mi sembrò odioso, quando, nella luce del giorno, egli solo fedelmente amato, sparve agli sguardi d'amore e davanti a me stette, nemico.

Quella luce che traditore mi ti mostrava,

a quella luce del giorno

volli sfuggire;

e laggiù in quella notte

te con me trarre,

dove la fine dell'illusione

il mio cuore mi prometteva;

dove dell'inganno il presagito

errore si sperdesse;

per libare a te colà

eterno amore,

te insieme con me

volli io consacrare alla morte.

xxxIV GS: «umfangen». 
TRISTAN $^{\text {Xxxv }}$

In deiner Hand

den süßen Trank, ${ }^{\text {Xxxvi }}$

als ich ihn erkannt

den sie mir bot;

als mir die Ahnung

hehr und gewiß

zeigte, was mir

die Sühne verhieß:

da erdämmerte mild

erhabner Macht

im Busen mir die Nacht;

mein Tag war da vollbracht.

ISOLDE

Doch ach! Dich täuschte

der falsche Trank,

daß dir von Neuem

die Nacht versank;

dem einzig am Tode lag,

den gab er wieder dem Tag.

TRISTAN

O Heil dem Tranke!

Heil seinem Saft!

Heil seines Zaubers

hehrer Kraft!

Durch des Todes Tor,

wo er mir floß,

weit und offen

er mir erschloß,

darin ich sonst ${ }^{\mathrm{xxxII}}$ nur träumend gewacht,

das Wunderreich ${ }^{\text {xxxviII }}$ der Nacht.

Von dem Bild in des Herzens

bergendem Schrein

scheucht' er des Tages

täuschenden Schein,

daß nacht-sichtig mein Auge

wahr es zu sehen tauge.

ISOLDE

Doch es rächte sich
TRISTANO

Nella tua mano

il dolce filtro

quand'io lo riconobbi

ch'ella m'offriva;

quando un presentimento

nobile, certo,

mostrò quel che a me

la riconciliazione prometteva,

allora sorse, come un dolce crepuscolo

di sublime potenza,

nel mio cuore la notte;

fu il mio giorno consumato.

ISOLDA

Pure, ahimè, t'illuse

il perfido filtro,

così che a te nuovamente

s'affondò la notte:

colui, che solo pensava alla morte,

il filtro donò nuovamente al giorno!

TRISTANO

Oh! benedetto quel filtro!

Benedetto il suo succo!

Benedetto della sua magia

il nobile potere!

A traverso la porta della morte,

là dove per me fu versato,

ampio ed aperto

esso mi schiuse

quello che io non avevo ancora visto che in sogno:

il reame meraviglioso della notte.

Dall'immagine, del cuore

nel celeste scrigno [racchiusa],

esso cacciò del giorno

l'ingannevole luce,

affinché il mio occhio veggente nella notte

valesse a contemplarla nella sua realtà.

ISOLDA

Pure si vendicò

xxxv Secondo le intenzioni di Wagner (da lui realizzate in diverse esecuzioni e confermate a Hermann Levi nel 1880) la sezione seguente del Tagesgespräch di Tristan e Isolde, fino al verso «Wie ertrag' ich's noch?» (corrispondente alle bb. 933-1036 della partitura) può essere tagliata.

xxxvI GS: «Tod».

xxxvII GS: «sonst ich».

xxxVIII GS: «Wonnereich». 
der verscheuchte Tag;

mit deinen Sünden

Rats er pflag:

was dir gezeigt

die dämmernde Nacht, an des Tag-Gestirnes

Königs-Macht

mußtest du's übergeben,

um einsam

in öder Pracht

schimmernd dort zu leben. -

Wie ertrug ich's nur?

Wie ertrag' ich's noch?

TRISTAN

$\mathrm{O}$ ! nun waren wir

Nacht-geweihte:

der tückische Tag, ${ }^{11}$

der Neid-bereite, trennen konnt' uns sein Trug, doch nicht mehr täuschen sein Lug.

Seine eitle Pracht,

seinen prahlenden Schein

verlacht, wem die Nacht

den Blick geweih't:

seines flackernden Lichtes

flüchtige Blitze

blenden uns nicht mehr. ${ }^{\mathrm{xx} x \mathrm{x}}$

Wer des Todes Nacht

liebend erschaut,

wem sie ihr tief

Geheimniß vertraut,

des Tages Lügen,

Ruhm und Ehr',

Macht und Gewinn,

so schimmernd hehr, wie eitler Staub der Sonnen sind sie vor dem zersponnen. ${ }^{\mathrm{XI}}$ il giorno cacciato;

coi tuoi peccati

egli prese consiglio;

quel che ti aveva mostrato

la notte crepuscolare,

alla della costellazione del giorno

regale potenza,

dovesti consegnare:

per solitario,

in deserta magnificenza

e splendidamente vivere in essa.

Come l'ho mai potuto sopportare?

Come lo sopporto ancora?

TRISTANO

Oh eravamo ormai

consacrati alla notte!

Il frodolento giorno,

pronto all'invidia,

ci poteva separare col suo inganno,

ma non più illudere con la sua menzogna!

La sua vana magnificenza,

il suo vanitoso bagliore

deride, colui al quale la notte

ha consacrato la vista!

Della sua luce vacillante

i lampi fuggitivi

non ci abbagliano più.

Chi la notte della morte

ha visto in amore;

colui al quale ella il suo profondo

segreto ha affidato,

le menzogne del giorno,

la gloria e l'onore,

la potenza e la ricchezza,

per quanto splendidi e nobili,

come vana polvere solare

davanti a lui si sono dissipati!

11 L'ultima parola prima del 'tempo lento' della scena d'amore (vedi nota 12) spetta a Tristan, che devia definitivamente la conversazione dal 'giorno' alla «santa notte» della morte e alla «beatitudine d'amore» (Liebes-Wonne) che vi si cela. Clarinetto e oboe reintroducono il motivo del Richiamo d'amore (es. 13) («In des Tages eitlem Wähnen»), mentre il motivo della Morte (es. 8) torna nella voce di Tristan («das Sehnen hin I zur heil'gen Nacht») e nell'orchestra in una riscrittura egemonizzata dalla sonorità del Tristanakkord (es. 1z) - una riscrittura che presto si consoliderà in una nuova forma (vedi $y$ dell'es. 17).

xxxix GS: «blenden nicht mehr I unsre Blicke».

XL. GS: seguono questi versi, che non sono stati musicati: «Selbst um der Treu' | und Freundschaft Wahn I dem treusten Freunde I ist's getan, I der in der Liebe I Nacht geschaut, I dem sie ihr tief I Geheimnis vertraut». 
In des Tages eitlem Wähnen

bleibt ihm ein einzig Sehnen,

das Sehnen hin

zur heil'gen Nacht,

wo ur-ewig,

einzig wahr

Liebes-Wonne ihm lacht.

(Tristan zieht Isolde sanft zur Seite auf eine Blumenbank nieder, senkt sich vor ihr auf die Knie und schmiegt sein Haupt in ihren Arm.)

BEIDE ${ }^{\mathrm{X} L \mathrm{I}}$

$\mathrm{O}$ sink hernieder, ${ }^{12}$

Nacht der Liebe,
Nella vana illusione del giorno

rimane a colui una sola aspirazione...

l'aspirazione laggiù,

verso la sacra notte,

dove ab aeterno,

unico vero,

a lui sorride la voluttà d'amore.

(Tristano trae Isolda dolcemente al suo fianco su d'un sedile fiorito, le si inginocchia davanti, e poggia il capo tra le sue braccia)

AMBEDUE

Oh scendi quaggiù, notte d'amore;

XLI GS: «(zu immer innigerer Umarmung auf einer Blumenbank sich niederlassend)».

12 Su un morbido tappeto accordale ritmato dagli archi divisi e con sordina, Tristan intona i suoi primi due versi su una melodia che scompone il Tristanakkord del preludio (es. 1z: ora notato come $\mathrm{Mib}_{3}-\mathrm{Fa}_{3}-\mathrm{Lab}_{3}$-Dob D $_{4}$ ), seguito da Isolde. È il principio del cosiddetto 'canto notturno' (Nachtgesang) dei due amanti, due strofe con coda in tempo «moderatamente lento" (Mäßig langsam) che gravitano attorno al Lab e danno il via al lungo "tempo lento' della scena. Il Nachtgesang attinge parte dei suoi materiali da Träume (Sogni): l'ultimo dei Fünf Gedichte für eine Frauenstimme mit Pianoforte-Begleitung WwV 91 (Wesendonck-Lieder), piccolo ciclo liederistico composto e rivisto tra il 1857 ed il 1858 da Richard Wagner su testi 'tristaneggianti' firmati da Mathilde Wesendonck. È interessante notare come Wagner intessa nel filo melodico vocale-strumentale della prima strofa anche il motivo del Giorno (es. 11), il cromatismo del Desiderio (es. 1y) e perfino allusioni a quello dello Sguardo (es. $2 y$ ). La seconda strofa (da "Barg im Busen») ripristina il tempo iniziale e (da «Herz an Herz dir») aggiunge ai materiali precedenti un'anticipazione del motivo del Sopore (Schlummermotiv: vedi $x$ nell'es. 17), e quella riscrittura a base di Tristanakkorde del motivo della Morte (es. 8) che fisserà la sua forma definitiva alla conclusione in forte della strofa ( «liebe-heiligstes Leben»: cfr. $y$ dell'es. 17). La coda del brano sfuma nel 'canto di veglia' (Wachtgesang) che la voce di Brangäne diffonde dallo spalto, in dialogo con due coppie di violini: una pagina costruita sui materiali del Nachtgesang sui quali l'ancella lascia fluttuare la sua lenta, ipnotica melodia (a sua volta modellata sugli intervalli del motivo del Giorno). I due amanti reagiscono agli avvertimenti di Brangäne con un 'canto dell'alba' (Morgenlied) in Solb («Lausch, Geliebter!»), su un impasto di archi in pianissimo, che introduce subito la forma definitiva del motivo del Sopore (es. 17x: una trasformazione dell'es. 4), a fianco della nuova veste del motivo della Morte (es. 17y).

ESEMPIO 17 (bb. 1266-1272)

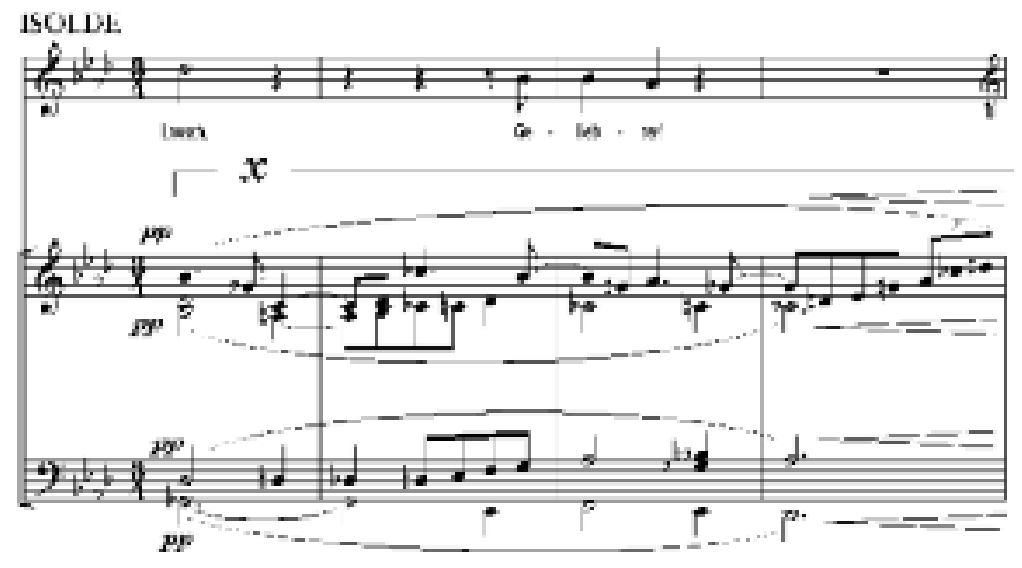


gib Vergessen,

daß ich lebe;

nimm mich auf

in deinen Schoß,

löse von

der Welt mich los!

TRISTAN $^{\text {XLII }}$

Verloschen nun

die letzte Leuchte;

ISOLDE

was wir dachten,

was uns deuchte, dona l'oblio

che io viva;

accoglimi

nel tuo seno;

scioglimi

via dal mondo!

TRISTANO

Spente ormai

le ultime luci;

ISOLDA

quel che noi pensammo,

quel che a noi parve;

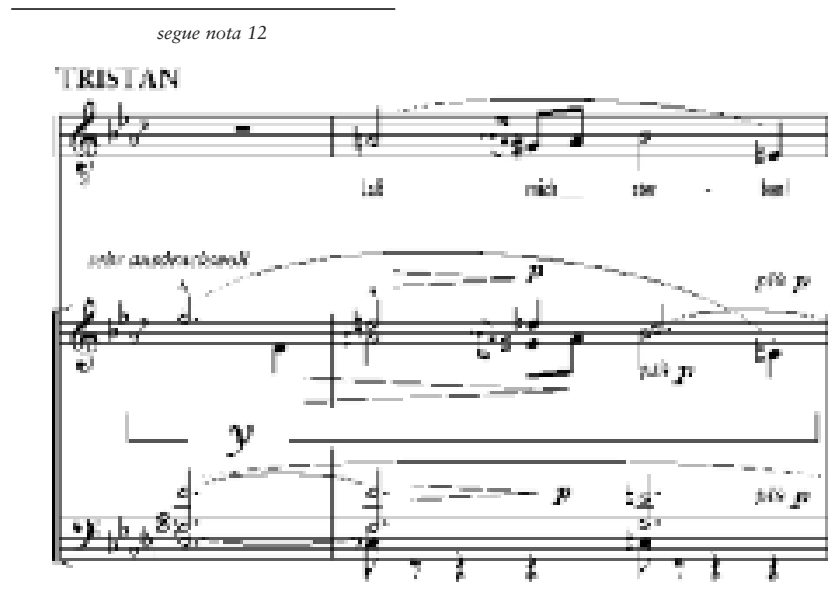

Dopo l'intermezzo della filosofica riflessione sulla «soave paroletta» $e$ (che l'orchestra accompagna con un fluido movimento dedotto dall'incipit dell'es. $17 x$ ), il tempo lento si chiude simmetricamente con un secondo canto a due («So starben wir»), seguito da una ripresa accorciata dell'avvertimento di Brangäne e del 'canto dell'alba' di Tristan e Isolde («Soll ich lauschen?»). «So starben wir» è il celeberrimo 'canto di morte' (Sterbelied) della coppia, in $\mathbf{8}$ sul tremolo impercettibile degli archi con sordina (es. 18), che 'ruota' su se stesso in progressioni e giocherà un ruolo cruciale nella scena conclusiva di Tristan (vedi nota 24).

ESEMPIO 18 (bb. 1377-1380)

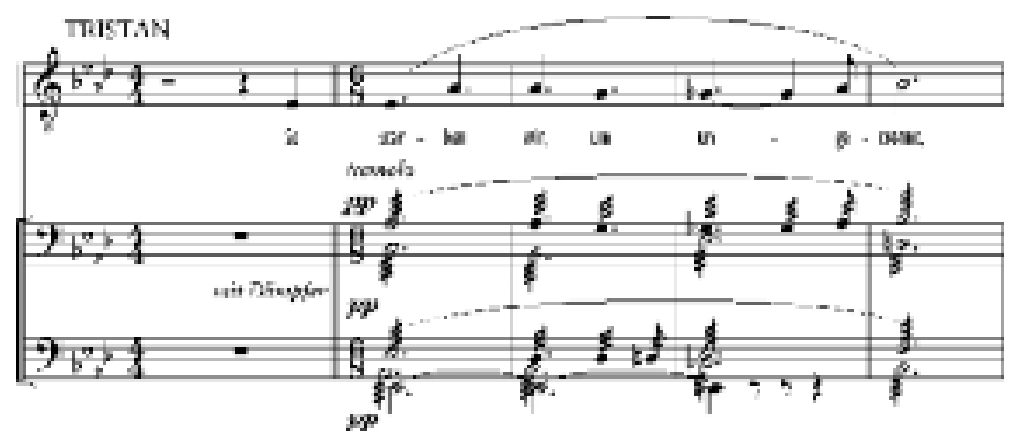

XLII GS: l'intera sequenza di versi fino a «hold bewußter Wunsch» è attribuita a entrambi i personaggi; si è provveduto a segnalare il cambio di personaggio in base alla partitura. 
TRISTAN

all' Gedenken,

ISOLDE

all' Gemahnen,

BEIDE

heil'ger Dämmrung

hehres Ahnen

löscht des Wähnens Graus

Welt-erlösend aus.

ISOLDE

Barg im Busen

uns sich die Sonne,

leuchten lachend

Sterne der Wonne.

TRISTAN

Von deinem Zauber

sanft umsponnen,

vor deinen Augen

süß zerronnen,

ISOLDE

Herz an Herz dir,

Mund an Mund,

TRISTAN

Eines Athems

Ein'ger Bund; -

BEIDE

bricht mein Blick sich

wonn'-erblindet,

erbleicht die Welt

mit ihrem Blenden:

ISOLDE

die uns ${ }^{\text {XLIII }}$ der Tag

trügend erhellt,

TRISTAN

zu täuschendem Wahn

entgegengestellt,

BEIDE

selbst - dann

bin ich die Welt,

wonne-hehrstes Weben

liebe-heiligstes Leben, ${ }^{\text {XLIV }}$
TRISTANO

ogni ricordo...

ISOLDA

ogni sovvenire,...

AMBEDUE

di un sacro crepuscolo

l'augusto presagio

l'orrore dell'illusione scioglie, liberando dal mondo.

ISOLDA

[Da poi che] s'è nascosto in cuore

a noi il sole,

splendono ridendo

stelle di voluttà.

TRISTANO

Dal tuo incanto

lievemente circonfuso,

davanti ai tuoi occhi

dolcemente perduto;

ISOLDA

il mio cuore sul tuo, sulla tua la mia bocca;

TRISTANO

di un solo respiro

unico vincolo;...

AMBEDUE

si smarrisce il mio sguardo

abbagliato dalla voluttà,

impallidisce il mondo

col suo barbaglio.

ISOLDA

Quel mondo, che il giorno

ingannevolmente illumina,

TRISTANO

per mentita illusione

a noi contrapposto,

AMBEDUE

io stesso, dunque,

sono quel mondo:

sacra trama di voluttà

santa vita di passione

\footnotetext{
XLIII GS: «mir».

xuv GS: «liebe-heiligstes Leben, I wonne-hehrstes Weben».
} 
nie-wieder-Erwachens

wahnlos

hold bewußter Wunsch.

(Tristan und Isolde versinken wie in gänzliche Entrücktheit, in der sie, Haupt an Haupt auf die Blumenbank zurückgelehnt, verweilen. $)^{\mathrm{XLV}}$

BRANGÄNE (unsichtbar, von der Höhe der Zinne)

Einsam wachend

in der Nacht,

wem der Traum

der Liebe lacht,

hab' der Einen

Ruf in Acht,

die den Schläfern

Schlimmes ahnt,

bange zum

Erwachen mahnt.

Habet Acht!

Habet Acht!

Bald entweicht die Nacht.

ISOLDE (leise)

Lausch, Geliebter!

TRISTAN (ebenso)

Laß mich sterben!

ISOLDE (allmählich sich ein wenig erhebend)

Neid'sche Wache!

TRISTAN (zurückgelehnt bleibend)

Nie erwachen!

ISOLDE

Doch der Tag

muß Tristan wecken?

TRISTAN (ein wenig das Haupt erhebend)

Laß den Tag

dem Tode weichen!

ISOLDE (nicht heftig)

Tag und Tod

mit gleichen Streichen

sollten unsre

Lieb' erreichen?

TRISTAN (sich mehr aufrichtend)

Unsre Liebe?

Tristans Liebe? del mai più svegliarsi

vigile,

dolce, consapevole volere.

(Tristano ed Isolda si perdono nella plenitudine del-

l'estasi, indugiando, abbandonati sul sedile fiorito, capo appoggiato a capo.)

VOCE DI BRANGANIA (invisibile, dall'alto della terrazza merlata)

Solitaria vigilante

nella notte;

colui, al quale il sogno

dell'amore sorride,

presti di una sola

al grido attenzione;

di colei che ai dormienti

presagisce il periglio,

ed ansiosamente al

risveglio li chiama.

Attenti!

Attenti!

Presto cede la notte.

ISOLDA (sommessamente)

Odi, mio caro!

TRISTANO (c.s.)

Lasciami morire!

ISOLDA (lentamente di poco sollevandosi)

Invidiosa vigilia!

TRISTANO (rimanendo supino)

Mai più svegliarsi!

ISOLDA

E pure il giorno

dovrà svegliare Tristano?

TRISTANO (sollevando un poco il capo)

Lascia che il giorno

ceda alla morte!

ISOLDA (senza irruenza)

Giorno e morte

con gli stessi colpi,

dovrebbero il nostro

amore colpire?

TRISTANO (sollevandosi maggiormente)

Il nostro amore?

L'amore di Tristano?

xLV GS: «(Mit zurückgesenkten Häuptern lange schweigende Umarmung Beider)». 
Dein' und mein',

Isoldes Liebe?

Welches Todes Streichen

könnte je sie weichen?

Stünd' er vor mir,

der mächt'ge Tod,

wie er mir Leib

und Leben bedroht, ${ }^{\text {XLVI }}$

die ich so willig

der Liebe ${ }^{\mathrm{XLVII}}$ lasse! -

wie wäre seinen Streichen

die Liebe selbst zu erreichen?

(Immer inniger mit dem Haupt sich an Isolde schmiegend)

Stürb' ich nun ihr,

der so gern ich sterbe,

wie könnte die Liebe

mit mir sterben?

Die ewig lebende

mit mir enden?

Doch stürbe nie seine Liebe, wie stürbe dann Tristan

seiner Liebe?

ISOLDE

Doch - unsre Liebe,

heißt sie nicht Tristan

und - Isolde?

Dieß süße Wörtlein: und,

was es bindet,

der Liebe Bund,

wenn Tristan stürb',

zerstört' es nicht der Tod?

TRISTAN (sehr ruhig)

Was stürbe dem Tod,

als was uns stört,

was Tristan wehrt

Isolde immer zu lieben,

ewig ihr nur ${ }^{\mathrm{XLVIII}} \mathrm{zu}$ leben?

ISOLDE

Doch, dieses Wörtlein: - und, ${ }^{\text {XLIX }}$
Il tuo e il mio?

L'amore d'Isolda?

Quale colpo di morte

potrebbe mai vincerlo?

Stesse avanti a me

la morte potente,

a me persona

e vita minacciando,

che così volentieri

ad amore io sacrifico;

come sarebbe ai suoi colpi

l'amore stesso raggiungibile?

(Sempre piu intimo, col capo stretto alla persona d'Isolda)

Morissi anche io d'amore, onde così volentieri io muoio,

come potrebbe l'amore

con me morire,

l'eterno vivente

con me finire?

Ma, se l'amore di lui non potrà mai morire, come potrebbe mai Tristano morire

al suo amore?

ISOLDA

Ma il nostro amore

non si chiama Tristano

e... Isolda?

Questa dolce paroletta: $e$

quel ch'essa congiunge,

questo vincolo d'amore,

se Tristano morisse,

non verrebbe distrutto dalla morte?

TRISTANO (con molta calma)

Che cosa soccomberebbe alla morte,

se non quel che ci disturba,

se non quel che impedisce a Tristano

di amare sempre Isolda,

e di vivere eternamente per lei?

ISOLDA

Pure se questa paroletta: $e, \ldots$

\footnotetext{
xIVI GS: «bedroht'».

xLVII GS: «der Liebe I so willig».

xLVIII GS: «nur ihr».

xuIX GS: «Doch das Wörtlein: und».
} 
wär' es zerstört,

wie anders als

mit Isoldes eignem Leben

wär' Tristan der Tod gegeben?

(Tristan zieht, mit bedeutungsvoller Gebärde, Isolde sanft an sich.)

TRISTAN

So starben wir,

um ungetrennt,

ewig einig,

ohne End',

ohn' Erwachen,

ohn' Erbangen, ${ }^{\mathrm{L}}$

namenlos

in Lieb' umfangen,

ganz uns selbst gegeben

der Liebe nur zu leben.

ISOLDE (wie in sinnender Entrücktheit zu ihm aufblikkend)

So stürben wir,

um ungetrennt -

TRISTAN

Ewig einig,

ohne End' -

ISOLDE

Ohn' Erwachen -

TRISTAN

Ohn' Erbangen -

ISOLDE

Namenlos

BEIDE

in Lieb' umfangen -

Ganz uns selbst gegeben, der Liebe nur zu leben! ${ }^{\mathrm{LI}}$

(Isolde neigt wie überwältigt das Haupt an seine Brust.)

BRANGÄNE (wie vorher)

Habet Acht!

Habet Acht!

Schon weicht dem Tag die Nacht. fosse annientata;

come altrimenti che

con la vita stessa d'Isolda,

potrebbe essere data la morte a Tristano?

(Tristano, con gesto pieno d'amore, attira Isolda dolcemente a sé.)

TRISTANO

Così siamo morti:

per inseparati,

eternamente congiunti,

senza fine,

senza risveglio,

senza sospetto,

ineffabilmente

presi in amore, a noi soli intenti, vivere d'amore.

ISOLDA (guardandolo come in estasi pensosa)

Così noi moriremmo:

per inseparati,...

TRISTANO

eternamente congiunti,

senza fine,...

ISOLDA

senza risveglio,...

TRISTANO

senza sospetto,...

ISOLDA

ineffabilmente

AMBEDUE

presi in amore, a noi soli intenti, vivere solo all'amore!

(Isolda come sopraffatta china il capo sopra il suo petto.)

BRANGANIA (come prima)

Attenti!

Attenti!

Già cede al giorno la notte.

\footnotetext{
${ }^{L}$ GS: «ohne Bangen».

LI GS: «TRISTAN | Ewig einig - I ISOLDE | Ohne End' - | TRISTAN | Ohn’ Erwachen - I ISOLDE | Ohne Bangen - | TRISTAN | Namenlos | in Lieb' umfangen - | ISOLDE | Ganz uns selbst gegeben, I der Liebe nur zu leben?».
} 


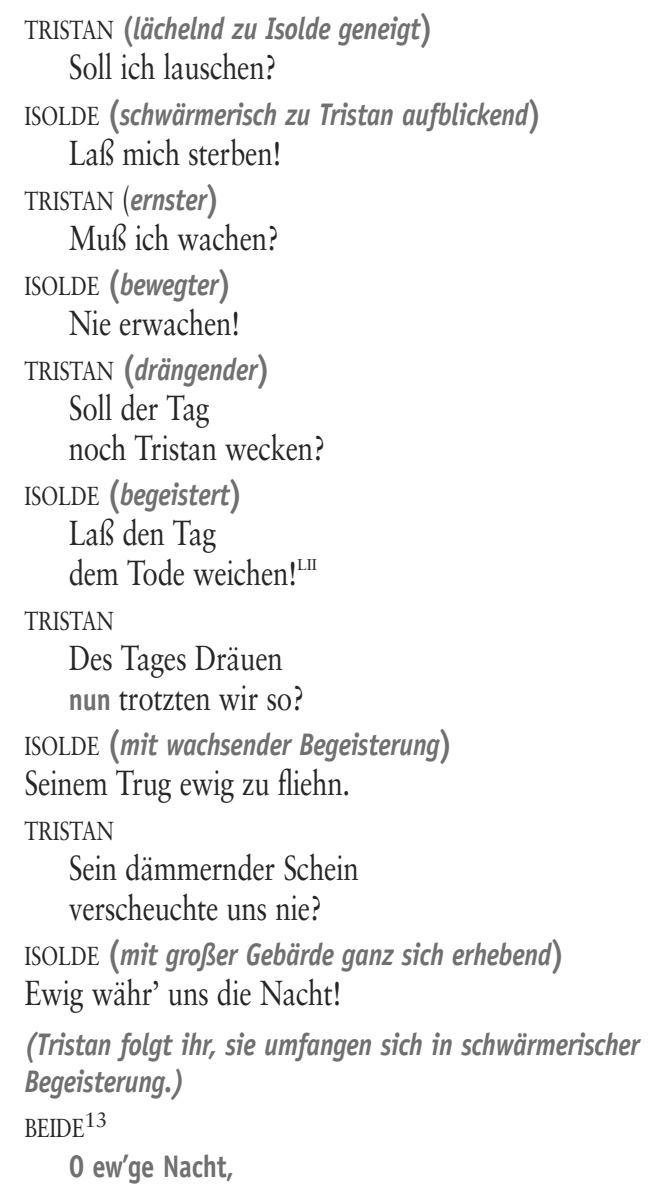

TRISTANO (chino su Isolda, e sorridente)

Debbo prestare ascolto?

ISOLDA (guardando Tristano con passione)

Lasciami morire!

TRISTANO (più gravemente)

Debbo vegliare?

ISOLDA (più animatamente)

Mai più svegliarsi!

TRISTANO (più incalzante)

Dovrà il giorno

ancora svegliare Tristano?

ISOLDA (esaltata)

Lascia che il giorno

ceda alla morte!

TRISTANO

La minaccia del giorno

così noi sfideremmo?

ISOLDA (con crescente esaltazione)

Alla sua frode per sempre fuggire!

TRISTANO

Il bagliore del suo crepuscolo

non ci caccerebbe più mai?

ISOLDA (alzandosi del tutto con gesto solenne)

Che la notte duri eterna per noi!

(Tristano la segue: essi si abbracciano con appassionata esaltazione.)

AMBEDUE

O notte eterna,

LII GS: seguono questi versi, che non sono stati musicati: «TRISTAN: Soll der Tod I mit seinen Streichen I ewig uns I den Tag verscheuchen? I IsOLDE: Der uns vereint, I den ich dir bot, I laß ihm uns weihn, I dem süßen Tod! I Mußte er uns I das eine Tor, I an dem wir standen, I verschließen; I zu der rechten Tür', I die uns Minne erkor, I hat sie den Weg nun gewiesen».

13 Preparata da una graduale intensificazione dinamica e agogica (cominciata a «Des Tages Dräuen»), ha inizio la sezione in tempo Allegro della scena d'amore (Sehr lebhaft und schnell: «molto vivace e veloce»), sulle note del nuovo motivo della Morte (es. 17y). La climax di tutta la scena coincide con uno sviluppo pressoché ininterrotto prima del 'canto di morte' (es. 18), poi del motivo della Beatitudine (es. 14), che cattura l' «ardente esaltazione» degli amanti abbracciati. Nell'insieme è riconoscibile una forma AA'B, imperniata sulla tonalità di Si: le due sezioni A (rispettivamente da «Wie sie fassen?» e da «traut allein») allineano una catena di progressioni sul "canto di morte', poi regolarmente ornate da una figura dolce di Trasfigurazione introdotta dal clarinetto (Verklärungsfigur: $x$ nell'es. 19), e si chiudono su una combinazione di motivo del Fato (es. $2 x$ ) e del Desiderio (es. 1y); infine, la tensione così accumulata si scarica sulla sezione B («endlos ewig | ein-bewußt»: l'acme dell'estasi), che raggiunge il parossimo in un lungo crescendo basato sul motivo della Beatitudine. Finché la musica si installa sulla dominante di Si, pronta a cadenzare sulla tonica, mentre il movimento si fa «ancora più incalzante» (Noch drängender). Ma il grido di Brangäne e l'ingresso di Kurwenal armato faranno deragliare violentemente la conclusione. 
süße Nacht! ${ }^{\text {LIII }}$

Hehr erhabne,

Liebes-Nacht!

ISOLDE ${ }^{\mathrm{LIV}}$

Wen du umfangen,

TRISTAN

wem du gelacht,

BEIDE

wie - wär' ohne Bangen

aus dir er je erwacht?

Nun banne das Bangen,

ISOLDE

holder Tod,

BEIDE

sehnend verlangter

Liebes-Tod!

In deinen Armen,

dir geweiht,

ur-heilig Erwarmen,

von Erwachens Not befreit.

TRISTAN

Wie sie ${ }^{\mathrm{LV}}$ fassen?

Wie sie lassen,

diese Wonne,

BEIDE

fern der Sonne,

fern der Tage

Trennungs-Klage?

ISOLDE

Ohne Wähnen dolce notte!

Augusta, sublime

notte d'amore!

ISOLDA

Colui che tu hai stretto,

TRISTANO

colui al quale hai sorriso,

AMBEDUE

come senza timore

si sveglierebbe mai da te?

Bandisci dunque il timore,

ISOLDA

o dolce morte,

AMBEDUE

0 ardentemente invocata

morte d'amore!

Nelle tue braccia,

a te sacri,

[quale] ardore santo ed antico, libero dall'angoscia del risveglio.

TRISTANO

Come comprenderla,

come lasciarla,

questa voluttà,

AMBEDUE

lontana dal sole,

lontana dal giornaliero

dolore della separazione?

ISOLDA

Senza illusione

segue nota 13

ESEMPIO 19 (bb. 1542-1545)

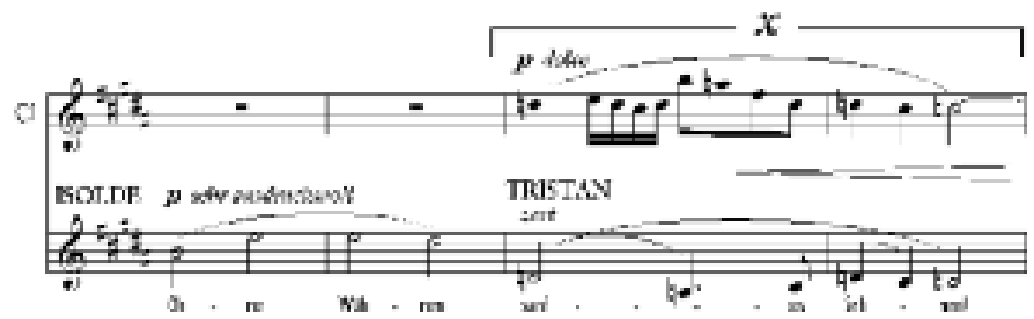

LIII GS: «O süße Nacht! | Ew’ge Nacht!».

LIV GS: l'intera sequenza di versi fino alla conclusione della scena è attribuita a entrambi i personaggi; si è provveduto a segnalare il cambio di personaggio in base alla partitura.

LV GS: «es». 
TRISTAN

sanftes Sehnen,

ISOLDE

ohne Bangen

TRISTAN

süß Verlangen;

ohne Wehen

BEIDE

hehr Vergehen,

ISOLDE

ohne Schmachten

BEIDE

hold Umnachten;

TRISTAN

ohne Meiden,

BEIDE

ohne Scheiden, ${ }^{\mathrm{LVI}}$

traut allein,

ewig heim,

in ungemess'nen Räumen

übersel'ges Träumen.

ISOLDE

Du Isolde,

Tristan ich,

nicht mehr Isolde!

TRISTAN

Tristan du,

ich Isolde,

nicht mehr Tristan! $!^{\mathrm{LVII}}$

ISOLDE

ohne Nennen,

ohne Trennen,

neu Erkennen,

neu Entbrennen;

BEIDE

endlos ewig

ein-bewußt:

heiß erglühter Brust

höchste Liebes-Lust!
TRISTANO

mite aspirare;

ISOLDA

senza timore

TRISTANO

dolce desiderare;

senza dolore

AMBEDUE

alto disciogliersi;

ISOLDA

senza languire,

AMBEDUE

grato annottare;

TRISTANO

senza distacco,

AMBEDUE

senza separazione, caramente soli, ad un focolare eterno, in interminati spazi, sovrumano sognare.

ISOLDA

Tu Isolda, io Tristano, non più Isolda!

TRISTANO

Tu Tristano, io Isolda, non più Tristano!

ISOLDA

Senza chiamarsi, senza separarsi, nuovo riconoscere, nuovo ardere;

AMBEDUE

senza fine eternamente, intimamente consci: cuore ardente come la fiamma, suprema voluttà d'amore!

LVI GS: «ohne Scheiden, I ohne Meiden».

IVII GS: «Du Isolde, I Tristan ich, I nicht mehr Tristan, I nicht Isolde;». 


\section{DRITTE SZENE}

(Sie verbleiben in verzückter Stellung. Brangäne stößt einen grellen Schrei aus.)

KURWENAL (stürzt mit entblößtem Schwerte herein) ${ }^{\mathrm{LVIII}}$

Rette dich, Tristan!

(Er blickt mit Entsetzen hinter sich in die Szene zurück. Marke, Melot und Hofleute [in Jägertracht], kommen aus dem Baumgange lebhaft nach dem Vordergrunde und halten entsetzt der Gruppe der Liebenden gegenüber an. ${ }^{\text {LIX }}$ Brangäne kommt zugleich von der Zinne herab, und stürzt auf Isolde zu. Diese, von unwillkürlicher Scham ergriffen, lehnt sich mit abgewandtem Gesicht auf die Blumenbank. Tristan, in ebenfalls unwillkürlicher Bewegung, streckt mit dem einen Arme den Mantel breit aus, so daß er Isolde vor den Blicken der Ankommenden verdeckt. In dieser Stellung verbleibt er längere Zeit, unbeweglich den starren Blick auf die Männer gerichtet, die in verschiedener Bewegung die Augen auf ihn heften. Morgendämmerung)

TRISTAN (nach längerem Schweigen)

Der öde Tag -

zum letzten Mal!

MELOT (zu Marke) Lx

Das sollst du, Herr, mir sagen,

ob ich ihn recht verklagt?

Das dir zum Pfand ich gab,

ob ich mein Haupt gewahrt?

Ich zeigt' ihn dir

in offner Tat:

Namen und Ehr'

hab' ich getreu

vor Schande dir bewahrt.

MARKE (nach tiefer Erschütterung, mit bebender Stim$m e)^{\mathrm{LXI}}$

Tatest du's wirklich?'14

\section{SCENA TERZA}

(Rimangono in atto estatico. Brangania lancia un grido lacerante.)

KURWENAL (entra precipitosamente con la spada sguainata)

Sálvati, Tristano!

(Egli guarda atterrito dietro di sé, verso la scena. Marke, Melot e cortigiani [in abito di cacciatori] s'avviano in fretta dal viale verso il davanti della scena, e si arrestano inorriditi di fronte al gruppo degli amanti. Brangania scende subito dalla terrazza merlata ed accorre presso Isolda. La quale, presa da inconsapevole pudore, si appoggia, volgendo il viso, sul sedile fiorito. Tristano, con movimento egualmente inconscio, apre con un braccio il mantello, cosi ch'egli nasconde Isolda alla vista dei venienti. In questa posizione rimane un tempo abbastanza lungo, immobile, lo sguardo rigido e fisso sugli nomini, che con vario contegno lo fissano alla loro volta. Primi albori.)

TRISTANO (dopo un silenzio piuttosto lungo)

L'ignobile giorno

per l'ultima volta!

MELOT (a Marke)

Questo, o signore, mi dirai, se io l'abbia con ragione accusato?

Se quel che in pegno io t'ho dato,

la mia testa, l'abbia salvata?

Io te l'ho mostrato

in fatto aperto:

nome ed onore

fedelmente io t'ho

dall'onta preservato.

MARKE (con profonda commozione e con voce tremante)

Questo veramente hai fatto?

\footnotetext{
IVIII GS: "(Man hört einen Schrei Brangänes, zugleich Waffengeklirr. - Kurwenal stürzt, mit gezücktem Schwerte zurückweichend, herein)».

LIX GS: «Unmittelbar folgen ihm, heftig und rasch, Marke, Melot und mehrere Hofleute, die den Liebenden gegenüber zur Seite anhalten, und in verschiedener Bewegung die Augen auf sie heften».

LX GS: aggiunge «der in sprachloser Erschütterung steht».

LXI GS: «(mit zitternder Stimme)».

14 Per il lamento di Marke Wagner immagina un fondo orchestrale essenziale, sparso di silenzi, realizzato dai soli archi privati del loro registro acuto (i violini per lo più tacciono), ai quali si uniscono quasi esclusivamente fa-
} 
Wähnst du das? -

Sieh ihn dort,

den Treusten aller Treuen;

blick auf ihn,

den freundlichsten der Freunde:

seiner Treue

frei'ste That

traf mein Herz

mit schmerzlichstem ${ }^{\text {LXII }}$ Verrat!

Trog mich Tristan,

sollt' ich hoffen,

was sein Trügen

mir getroffen,

sei durch Melots Rat

redlich mir bewahrt?

TRISTAN (krampfhaft heftig)

Tags-Gespenster!

Morgen-Träume -

täuschend und wüst -

entschwebt, entweicht!

MARKE (mit tiefer Ergriffenheit)

Mir - dies?
Questo tu pensi?

Eccolo là,

il più fido di tutti i fidi;

guardalo

il più amico di tutti gli amici:

della sua fede

l'atto più libero

ha colpito il mio cuore

col più doloroso tradimento!

Se mi tradiva Tristano

dovevo io sperare,

che il male dal suo tradimento

a me fatto,

dal consiglio di Melot

mi fosse riparato?

TRISTANO (con violenza spasmodica)

Spettri del giorno!

Sogni del mattino

illusori ed orridi!

Sparite! Andatevene!

MARKE (con profondo turbamento)

A me questo?

segue nota 14

gotti e corni. A dividere la scena con Marke è invece il clarinetto basso, sorta di 'doppio' strumentale della voce addolorata del re tradito, che articola il lamento in tre parti. È questo strumento a prendere la parola per primo, con il profilo della figura dell'Afflizione (Trauerfigur: es. 20), discendente e cromatico come quello del Dolore (es. $1 x)$, che si aggira vanamente su se stesso un po' come le linee contorte di basso della frustrazione di Wotan nell'Anello del Nibelungo.

ESEMPIO 20 (bb. 1689-1696)

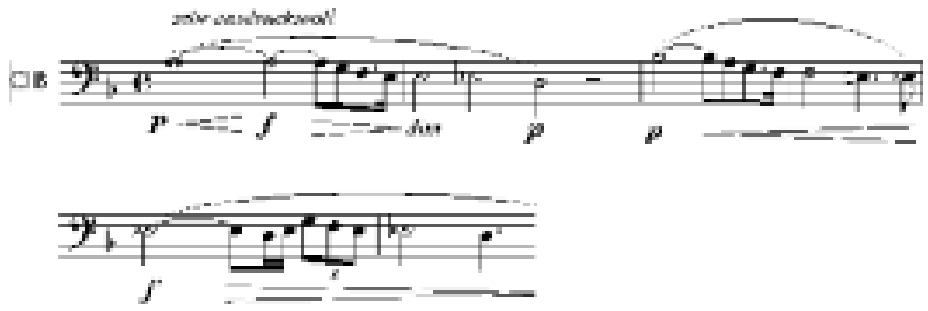

Ed è sempre il clarinetto basso a inaugurare la parte centrale con il Markemotiv (o 'motivo di Marke'), subito ceduto alla voce del re (es. 21: «Wozu die Dienste»). Nella voce dello strumento i due motivi si fondono, ed è appunto con la combinazione Trauerfigur + Markemotiv che il clarinetto basso vara la parte finale del lungo monologo, che conferma la tonica di re («Nun, da durch solchen»).

ESEMPIO 21 (bb. 1758-1760)

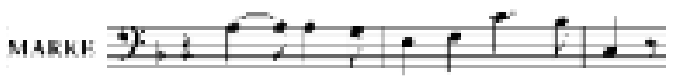

LXII GS: «feindlichstem». 


$$
\begin{aligned}
& \text { Dies -, Tristan, - mir? - } \\
& \text { Wohin nun Treue, } \\
& \text { da Tristan mich betrog? } \\
& \text { Wohin nun Ehr' } \\
& \text { und echte Art, } \\
& \text { da aller Ehren Hort, } \\
& \text { da Tristan sie verlor? } \\
& \text { Die Tristan sich } \\
& \text { zum Schild erkor, } \\
& \text { wohin ist Tugend } \\
& \text { nun entflohn, } \\
& \text { da meinen Freund sie flieht? } \\
& \text { da Tristan mich verriet? }
\end{aligned}
$$

(Schweigen. - Tristan senkt langsam den Blick zu Boden; in seinen Mienen ist, wäbrend Marke fortfährt, zunehmende Trauer zu lesen)

Wozu die Dienste

ohne Zahl,

der Ehren Ruhm, der Größe Macht,

die Marken du gewannst, mußt' Ehr' und Ruhm, Größe und Macht, mußte die Dienste ohne Zahl

dir Markes Schmach bezahlen?

Dünkte zu wenig dich mein ${ }^{\text {IXIII }}$ Dank, daß, was du mir erworben, Ruhm und Reich, ich $^{\text {LXIV }}$ zu Erb' und Eigen dir gab?

$D{ }^{\mathrm{LXV}}$ kinderlos einst

schwand sein Weib, so liebt' er dich, daß nie aufs Neu'

sich Marke wollt' vermählen.

Da alles Volk

zu Hof und Land

mit Bitt' und Dräuen

in ihn drang,
Questo, Tristano, a me?

Dove, dunque, la fede, se m’è Tristano infedele?

Dove, dunque, l'onore, ed il puro costume, poiché il fiore di ogni onore, Tristano, l'ha perduto?

Quella che a sé Tristano

scelse per scudo,

dov'è quella virtù

ora fuggita,

poiché ella fugge il mio amico, e m'ha tradito Tristano?

(Silenzio. - Tristano china leggermente lo sguardo al suolo; nel suo viso, mentre Marke prosegue, si legge un crescente dolore)

A che i servigi senza numero, degli onori la gloria della grandezza la potenza, che tu acquistasti a Marke; se doveva onore e gloria grandezza e potenza, se doveva i servigi senza numero a te l'onta di Marke pagare?

Così poco ti parve

la mia riconoscenza; che quel che tu mi avevi acquistato, gloria e regno, io ti donassi in eredità?

Poiché senza figli un giorno morì la sua donna, così egli t'amò che giammai più volle Marke toglier moglie. Come tutto il popolo, a corte ed in paese, con preghiere e minacce su di lui premé

\footnotetext{
LXIII GS: «sein».

LXIV GS: «er».

LXV GS: «Dem».
} 
die Königin dem Lande, ${ }^{\text {LXVI }}$

die Gattin sich zu kiesen;

da selber du

den Ohm beschworst,

des Hofes Wunsch,

des Landes Willen

gütlich zu erfüllen:

in Wehr wider ${ }^{\text {LXVII }}$ Hof und Land,

in Wehr selbst gegen dich,

mit List und Güte ${ }^{\text {LXVIII }}$

weigert' er sich,

bis, Tristan, du ihm drohtest

für immer zu meiden

Hof und Land,

würdest du selber

nicht entsandt,

dem König die Braut zu frei'n.

Da ließ er's denn so sein. -

Dies wundervolle ${ }^{\text {IXIX }}$ Weib,

das mir dein Mut gewann, ${ }^{\text {LXx }}$

wer durft' es sehen,

wer es kennen,

wer mit Stolze

sein es nennen,

ohne selig sich zu preisen?

Der mein Wille

nie zu nahen wagte,

der mein Wunsch

Ehrfurcht-scheu entsagte, die so herrlich

hold erhaben

mir die Seele

mußte laben,

trotz - Feind und Gefahr,

die fürstliche Braut

brachtest du mir dar.

Nun, da durch solchen

Besitz mein Herz

du fühlsamer schufst

als sonst dem Schmerz, perché una regina al paese,

una sposa a sé scegliesse;

come tu stesso

scongiurasti lo zio,

che il desiderio della corte,

e la volontà del paese

benignamente adempisse:

in guerra contro corte e paese,

in guerra contro te stesso,

con accortezza e bontà

egli si rifiutò,

finché, Tristano, tu lo minacciasti, di abbandonare per sempre

e corte e paese,

se tu stesso non fossi

inviato

a cercare al re la fidanzata;

allora soltanto egli consentì.

Questa meraviglia di donna

che il tuo coraggio a me conquistò, chi la potrebbe vedere,

chi conoscere,

chi con orgoglio

chiamarla sua,

senza ritenersi beato?

Colei alla quale la mia volontà

non ha mai osato accostarsi, colei alla quale il mio desiderio

pieno di sacro rispetto rinunziava,

colei che così generosa

dolce, sublime,

a me l'animo

doveva confortare,

sfidando nemico e pericolo,

la regale sposa,

tu a me portasti.

Ora, poiché con un tale

possesso il mio cuore

sensibile rendesti più

che mai al dolore,

\footnotetext{
LXVI GS: «Reiche».

LXVII GS: "gegen».

LxVIII GS: «mit Güt' und List».

LXIX GS: "wunderhehre».

LXX GS: «erwarb».
} 
dort wo am weichsten

zart und offen,

würd' ich ${ }^{\mathrm{LXXI}}$ getroffen,

nie zu hoffen,

daß je ich könne genesen, ${ }^{\text {LXXII }}$

warum so sehrend

Un-seliger,

dort - nun mich verwunden?

Dort mit der Waffe

quälendem Gift,

das Sinn und Hirn

mir sengend versehrt;

das mir dem Freund

die Treue verwehrt,

mein offnes Herz

erfüllt mit Verdacht,

daß ich nun heimlich

in dunkler Nacht

den Freund lauschend beschleiche, meiner Ehren End' erreiche?

Die kein Himmel erlöst, warum - mir diese Hölle?

Die kein Elend sühnt, warum - mir diese Schmach?

Den unerforschlich tief ${ }^{\mathrm{LXXIII}}$ geheimnißvollen Grund, wer macht der Welt ihn kund?

TRISTAN (das Auge mitleidig zu Marke erhebend) ${ }^{15}$

O König, das -

kann ich dir nicht sagen;

und was du frägst,

das kannst du nie erfahren. - là dove, se nel punto più tenero

in modo delicato e aperto,

io fossi stato colpito,

non sarebbe stata speranza

ch'io potessi mai più guarire;

perché così pungente,

sciagurato,

proprio là m'hai dunque ferito?

Colà col d'un'arma

torturante veleno,

che spirito e cervello

mi brucia consumando;

e che verso l'amico mi

impedisce la fede,

ed il mio cuore aperto

riempie di sospetto;

così che ora segretamente

nell'ombra della notte,

in agguato mi pongo per spiare l'amico,

e per ottenerne la fine del mio onore?

Questo, che nessun paradiso discioglierà, perché a me quest'inferno?

Questa, che nessun supplizio espierà, perché a me quest'onta?

Questo insondabile e profondo

e misterioso abisso

chi lo rivelerà al mondo?

TRISTANO (alzando gli occhi pieni di compassione verso Marke)

$\mathrm{O}$ re, questo

non posso io dirti;

e quel che tu chiedi

tu non potrai mai sapere.

LXXI GS: «es».

LXXII GS: "gesunden».

LXXIII GS: "Den unerforschlich | furchtbar tief».

15 Le parole della laconica risposta di Tristan sono interpolate nella ripresa letterale - ben altrimenti eloquente - del tema del Desiderio (il Sehnsuchtsthema, ossia il primo tema del preludio all'atto primo), ristrumentato per soli legni e corni. Tema che viene 'congelato' all'altezza della prima battuta dell'es. 2, per lasciare il posto al motivo del Sopore (es. 17x). È il momento del congedo degli amanti ( Wohin nun Tristan scheidet»), due strofe affini (la prima cantata da Tristan, la seconda da Isolde), nelle quali si infiltrano memorie musicali dal tempo lento della scena d'amore. Il tempo torna Lebhaft («vivace») e l'orchestra si riempie di scalette febbrili quando Melot estrae la spada e affronta l'amico di un tempo. A portare a conclusione la scena è una coda in diminuen$d o$, nella quale le prime quattro note del motivo di Marke (es. 21) si rincorrono dolorosamente verso il grave per un paio di ottave sopra la triade tenuta di tonica di re, fino a spegnersi nel più piano nei corni. Ma è una violenta triade di re nel tutti dell'orchestra a siglare l'atto. 
(Er wendet sich zu Isolde, die sehnsüchtig zu ihm aufblickt) ${ }^{\mathrm{LXXIV}}$

Wohin nun Tristan scheidet, willst du, Isold', ihm folgen?

Dem Land, das Tristan meint, der Sonne Licht nicht scheint:

es ist das dunkel

nächt'ge Land,

daraus die Mutter

mich entsandt, ${ }^{\text {LXXV }}$

als, den im Tode

sie empfangen,

im Tod sie ließ

an das ${ }^{\mathrm{LXXVI}}$ Licht gelangen.

Was, da sie mich gebar, ihr Liebesberge war, das Wunderreich der Nacht, aus der ich einst erwacht, das bietet dir Tristan,

dahin geht er voran.

Ob sie ihm folge

treu und hold,

das sag' ihm nun Isold'.

ISOLDE

Als ${ }^{\text {LXXVII }}$ für ein fremdes Land

der Freund sie einstens warb, dem Un-holden

treu und hold,

mußt' Isolde folgen.

Nun führst du in dein Eigen, dein Erbe mir zu zeigen;

wie flöh' ich wohl das Land,

das alle Welt umspannt?

Wo Tristans Haus und Heim,

da kehr' Isolde ein:

auf dem sie folge

treu und hold,

den Weg nun zeig Isold'!

(Tristan neigt sich langsam über sie und küßt sie sanft auf die Stirn. Melot fährt wütend auf.)
(Si volge verso Isolda, che lo guarda con passione)

Dove Tristano ora se n'andrà, vuoi tu seguirlo, Isolda?

Alla terra, a cui pensa Tristano, non luce luce di sole:

è dessa l'oscura

notturna terra,

onde mia madre

mi inviò,

quando, me che in morte

aveva concepito,

in morte ella fece

ch'io giungessi alla luce.

Quel che, quando mi partorì

era il suo asilo d'amore,

il mirabile regno della notte,

ond'io un giorno mi svegliai;

quello a te offre Tristano,

colà egli ti precede:

se lo seguirà

dolce e fedele,...

questo ora gli dica Isolda.

ISOLDA

Quando per una terra straniera

l'amico un giorno la richiese, lui non benigno, fedele e benigna

dovette Isolda seguire.

Ora tu mi conduci nel tuo regno,

per mostrarmi il tuo retaggio;

come fuggirei io il paese

che comprende tutti i mondi?

Dov'è la casa ed il focolare di Tristano,

là torni Isolda:

la via ov'ella lo segua

dolce e fedele;

la via mostra ad Isolda!

(Tristano si curva lentamente su di lei e la bacia lievemente sulla fronte. Melot balza in furore.)

\footnotetext{
LXXIV GS: «(Er wendet sich seitwärts zu Isolde, welche die Augen sehnsüchtig zu ihm aufgeschlagen hat)».

LXXV GS: «einst mich sandt'».

LXXVI GS: «Zum».

LXXVII GS: «Da».
} 
MELOT (das Schwert ziehend)

Verräter! Ha!

Zur Rache, König!

Duldest du diese Schmach?

TRISTAN (zieht sein Schwert und wendet sich schnell um)

Wer wagt sein Leben an das meine?

(Er beftet den Blick auf Melot)

Mein Freund war der;

er minnte mich hoch und teuer:

um Ehr' und Ruhm

mir war er besorgt wie keiner.

Zum Übermut

trieb er mein Herz:

die Schaar führt' er,

die mich gedrängt,

Ehr' und Ruhm mir zu mehren,

dem König dich zu vermählen. -

Dein Blick, Isolde,

blendet auch ihn:

aus Eifer verriet

mich der Freund

dem König, den ich verriet. -

(Er dringt auf Melot ein)

Wehr dich, Melot!

(Als Melot ihm das Schwert entgegenstreckt, läßt Tristan das seinige fallen und sinkt verwundet in Kurwenals Arme. Isolde stürzt sich an seine Brust. Marke bält Melot zurück. - Der Vorhang fällt schnell.)
MELOT (traendo la spada)

Traditore! Ah!

Vendetta, o re!

Soffriresti tu un'onta simile?

TRISTANO (trae la spada e si volta bruscamente)

Chi osa rischiare la propria vita contro la mia?

(Figge lo sguardo su Melot)

Costui era mio amico;

egli assai e caramente m'amava,

del mio onore e della mia gloria

come nessun altro per me si curava.

Alla tracotanza

egli spingeva il mio cuore:

la schiera egli guidava

che mi premeva,

per crescermi gloria ed onore,

affinché io ti sposassi al re.

Il tuo sguardo, o Isolda

anche lui ha abbagliato,

per gelosia ha tradito

me, l'amico

presso il re, che io ho tradito.

(Si precipita su Melot)

Difenditi, Melot!

(Nel momento in cui Melot gli oppone la spada, Tristano lascia cadere la sua, e cade ferito nelle braccia di Kurwenal. Isolda si precipita al suo petto. Marke trattiene Melot. - La tela cala rapidamente.) 


\section{DRITTER AUFZUG}

\section{ERSTE SZENE ${ }^{16}$}

(Burggarten. Zur einen Seite hohe Burggebäude, zur anderen eine niedrige Manerbrüstung, von einer Warte unterbrochen; im Hintergrunde das Burgthor. Die Lage ist auf felsiger Höhe anzunehmen; durch Öffnungen blickt man auf einen weiten Meereshori-

\section{SCENA PRIMA}

(Giardino d'un castello. Da un lato alti muri, dall'altro, un basso parapetto, interrotto da una torre di vedetta; in fondo, la porta del castello stesso. Si immagina che esso sia situato sull'alto di una roccia; a traverso i vani, la vista si stende su un ampio oriz-

16 Il Mäßig langsam («moderatamente lento») che inaugura l'atto terzo è probabilmente la pagina più nota di una sorta di 'micro-filone' interno al Musikdrama wagneriano, ossia quella del 'preludio di desolazione'; filone che include almeno il corrispondente preludio di Parsifal e l'introduzione/transizione alla fuga di Siegmund e Sieglinde nell'atto secondo di Walküre. Affine, nei tre casi citati, è la strategia strumentale: la quarta corda dei violini dà l'avvio a un melodizzare 'desolato', scavato sul registro grave degli altri archi. Affine, soprattutto, è la peculiare strategia che li accomuna: la sorpresa di un'alterazione radicale di un filo musicale particolarmente importante del tessuto leitmotivico. L'atto si apre infatti su una sconcertante riscrittura diatonica in fa del motivo cromatico del Desiderio (es. 1y):

ESEMPIO 22 (III, bb. 1-4)

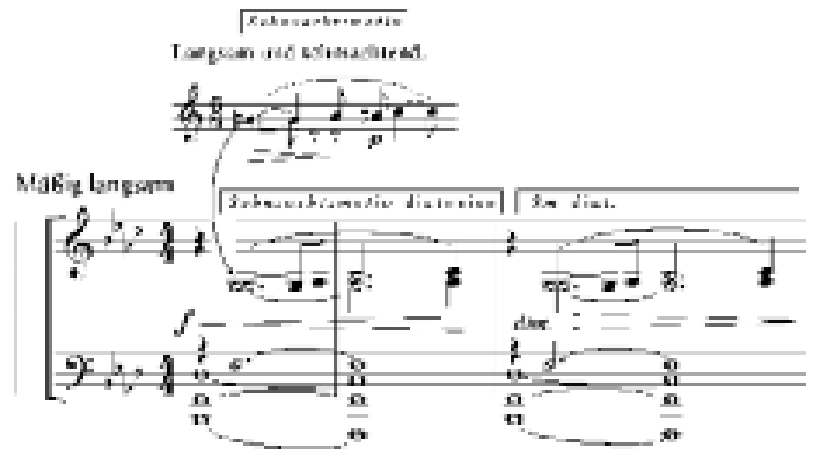

L'es. 22 si sfalda poi in una successione di terze e tritoni (es. 23), che - come scriveva Maurice Kufferath alla fine dell'Ottocento - «lasciano una singolare impressione di vuoto e di solitudine [une singulière impression de vide et de solitude]; pare di trovarsi dinanzi all'infinito dell'oceano inflessibile, mentre lo sguardo interroga invano il triste orizzonte".

ESEMPIO 23 (bb. 5-8)

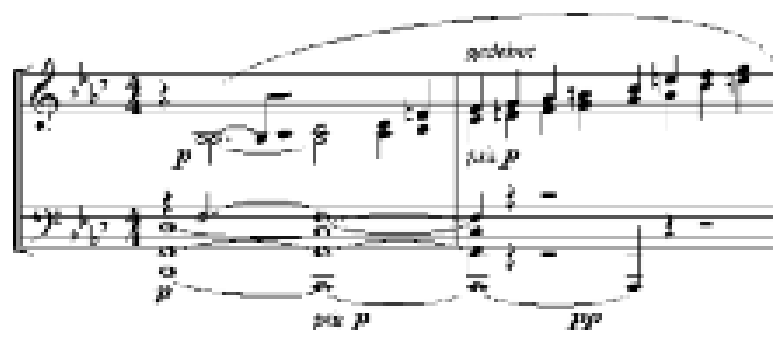

Introdotto sempre piano da corni e violoncelli, udiamo poi il motivo della Privazione d'amore (Liebesentbehrungsmotiv: es. 24), caratterizzato da una mistura di cromatismo e consonanza che avrà grandi sviluppi in Parsifal. 
zont. Das Ganze macht den ${ }^{\mathrm{T}}$ Eindruck der Herrenlosigkeit, übel gepflegt, hie und da schadhaft und bewachsen. Im Vordergrunde, an der inneren Seite, liegt, unter dem Schatten einer großen Linde, Tristan, auf einem Rubebette schlafend, wie leblos ausgestreckt. Zu Häupten ihm sitzt Kurwenal, in Schmerz über ibn hingebeugt, und sorgsam seinem Atem lauschend. - Von der Außenseite her hört man, beim Aufziehen des Vorhanges, einen Hirtenreigen, sebnsüchtig und traurig auf einer Schalmei geblasen. Endlich erscheint der Hirt selbst über der Mauerbrüstung mit dem Oberleibe, und blickt teilnehmend herein.)

$$
\begin{aligned}
& \text { HIRT (leise) } \\
& \text { Kurwenal! He! - } \\
& \text { Sag, Kurwenal! - } \\
& \text { Hör doch, Freund! }
\end{aligned}
$$

zonte di mare. L'insieme dà l'impressione della mancanza dei padroni; mal mantenuto, qua e là danneggiato e coperto d'erbe. Sul davanti della scena, nella parte interna, giace Tristano sotto l'ombra d'un gran tiglio, dormiente sopra un divano, steso come un corpo esanime. Al suo capezzale siede Kurwenal dolorosamente curvo su di lui, in atto di spiare con ansia il suo respiro. - Dalla parte esterna si ode, all'alzarsi del sipario, una danza pastorale, nostalgicamente e tristemente suonata da una zampogna. Infne appare il pastore con la parte superiore del corpo al di sopra del parapetto del muro e guarda dentro con curiosità).

IL PASTORE (sommessamente)

Kurwenal! Ehi!

Di', Kurwenal!

Senti, dunque, amico!

segue nota 16

ESEMPIO 24 (bb. 11-13)

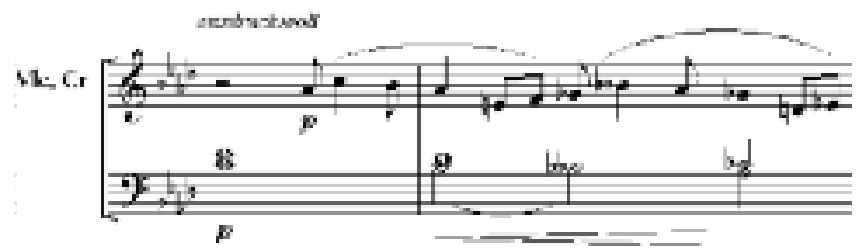

Gli ess. 22-24 provengono in realtà da Im Treibhaus (Nella serra), il terzo dei già citati Wesendonck-Lieder (vedi nota 12), indicato da Wagner stesso come Studie zu «Tristan und Isolde». I versi 'notturni' della Wesendonck compiangevano il «destino» delle «misere piante» strappate al luogo natio e confinate tra le pareti di una serra inospitale: a queste piante ora l'atto terzo del dramma di Wagner dà un nome ed un volto - quelli del Tristan visionario strappato ad Isolde e condotto morente in Bretagna. Su questi motivi Wagner imbastisce l'introduzione strumentale: al levarsi del sipario, udiamo da dietro le quinte il «suono nostalgico e triste» della cornamusa (in realtà, un corno inglese). Mentre l'orchestra tace, lo strumento suona la alte Weise, l'«antica canzone» (ancora in fa: es. 25) che sarà riconosciuta da Tristan:

ESEMPIO 25 (bb. 52-57)

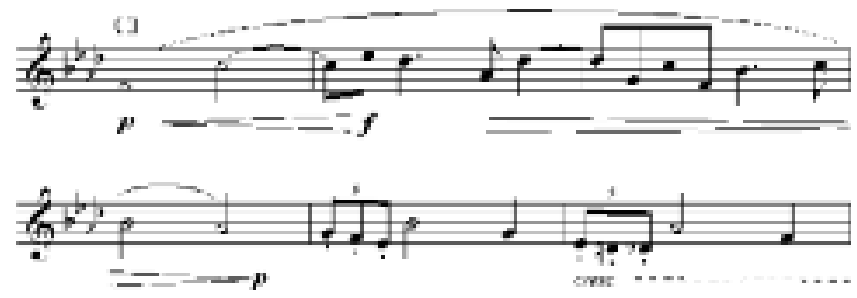

La successiva conversazione tra il pastore e Kurwenal conferma il fa come tonalità di controllo e vede il ritorno della desolazione strumentale del preludio: l'azione scenica viene sorretta dallo stesso tessuto motivico-orchestrale di una cinquantina di battute che abbiamo udito prima del sipario (sia pure, s'intende, con tutte le sostituzioni, le soppressioni, i dislocamenti resi necessari dal dramma).

I GS: «einen». 
(Kurwenal wendet ein wenig das Haupt nach ihm)

Wacht er noch nicht?

KURWENAL (schüttelt traurig mit dem Kopf)

Erwachte er,

wär's doch nur

um für immer zu verscheiden,

erschien zuvor

die Ärztin nicht,

die einz'ge, die uns hilft.

Sah'st du noch nichts?

Kein Schiff noch auf der See? -

HIRT

Eine and're Weise

hörtest du dann,

so lustig als ich sie nur ${ }^{\text {III }}$ kann.

Nun sag' auch ehrlich,

alter Freund:

was hat's mit unsrem Herrn?

KURWENAL

Laß die Frage; -

du kannst's doch nie erfahren. -

Eifrig späh,

und siehst du ein ${ }^{\text {IV }}$ Schiff,

$\mathrm{so}^{\mathrm{V}}$ spiele lustig und hell!

HIRT (sich wendend und mit der Hand über'm Auge, nach dem Meer spähend)

Öd' und leer das Meer! -

(Er setzt die Schalmei an den Mund und entfernt sich blasend) $)^{\mathrm{VI}}$

TRISTAN (nach langem Schweigen, obne Bewegung, dumpf)

Die alte Weise -17

was weckt sie mich?

(Die Augen aufschlagend und das Haupt wendend)

Wo - bin ich?

II GS: «(Da Kurwenal das Haupt nach ihm wendet)».

III GS: «wie ich sie».

IV GS: «das».

v GS: «dann».

VI GS: «(Er setzt die Schalmei an und verschwindet blasend: etwas ferner hört man längere Zeit den Reigen.)».

17 La ripetizione dell' «antica canzone» del corno inglese desta infine Tristan. Ma è un risveglio apparente, come la musica esprime con ineguagliabile efficacia: Tristan non rientra affatto nel mondo del 'giorno', rappresentato invece dal fido Kurwenal. Che non ha capito granché di quanto è successo al suo signore, e torna qui a parlare con entusiasmo l'unico linguaggio che conosca - quello appunto del 'giorno' e delle salde architetture dei suoi
(Kurwenal volge un poco il capo verso di lui)

Non si sveglia ancora?

KURWENAL (scuote tristemente il capo)

Se si svegliasse,

sarebbe soltanto

per lasciarci per sempre:

a meno che prima non apparisse

la sanatrice,

la sola, che ci può soccorrere...

Non hai visto ancora niente?

Nessun vascello ancora sul mare?

IL PASTORE

Un'altra aria

sentiresti allora,

così gaia, come meglio io posso.

Ora dimmi sinceramente,

mio vecchio amico:

che è successo al nostro signore?

KURWENAL

Lascia la domanda:

non potrai mai saperlo.

Scruta diligentemente,

e se tu vedi un naviglio,

suona un'aria gaia e serena!

IL PASTORE (voltandosi e scrutando verso il mare con la mano sugli occhi)

Deserto e vuoto il mare!

(Egli accosta la zampogna alle labbra e si allontana suonando)

TRISTANO (dopo un lungo silenzio, immobile, cupo)

La vecchia aria;...

a che ella mi desta?

(Aprendo gli occhi e volgendo un poco il capo)

Dove sono? 


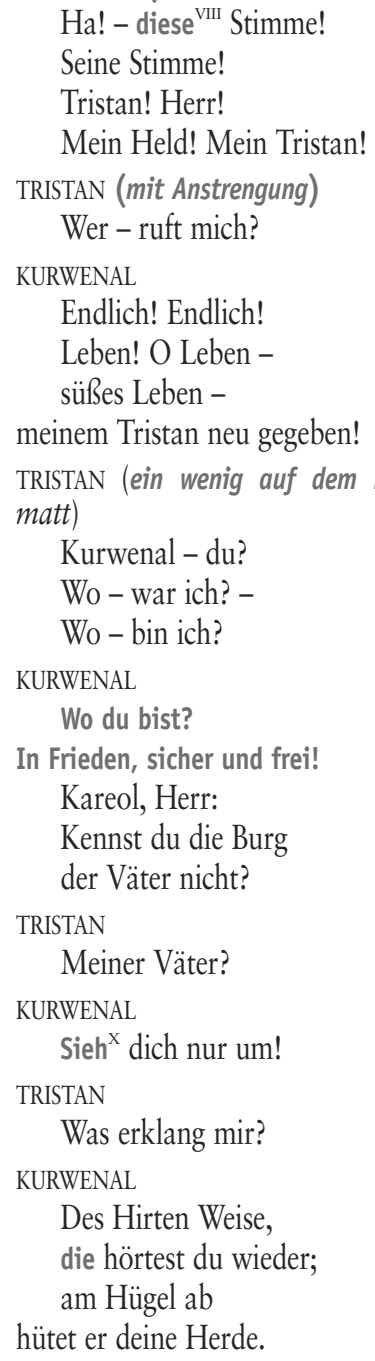

Des Hirten Weise, die hörtest du wieder; am Hügel $a b$ hütet er deine Herde.

\author{
KURWENAL (sobbalza, spaventato) \\ Ah! quella voce! \\ La sua voce! \\ Tristano! Signore! \\ Mio eroe! Mio Tristano \\ TRISTANO (a fatica) \\ Chi mi chiama? \\ KURWENAL \\ Finalmente! Finalmente! \\ Vita, o vita! \\ Dolce vita, \\ al mio Tristano nuovamente donata!
}

TRISTANO (alzandosi un poco sul suo giaciglio, esausto)
Kurwenal?... Tu?
Dov'ero?
Dove sono?

KURWENAL

Dove sei?

In pace, libero e sicuro!
Kareol, signore:
non conosci la rocca
degli avi?

TRISTANO

Dei miei avi?

KURWENAL

Guàrdati semplicemente intorno! TRISTANO

Che è che mi suona?
KURWENAL
L'aria del pastore
hai udito nuovamente;
lungo il pendio della collina
guarda i tuoi greggi.

segue nota 17

Lieder. Kurwenal cerca così prima di imbastire una gioiosa forma vocale-strumentale per celebrare Kareol, e poi («Nach Kornwall») ci fa risentire perfino un accenno del canto derisorio su Morold dell'atto primo (cfr. nota 3). Invano: la sua 'musica del giorno' è continuamente - e quanto emblematicamente! - compromessa da Tristan, con i suoi interventi che assomigliano (secondo Alfred Lorenz) a delle pause sognanti, senza tempo, interpolate nel flusso della musica rigidamente scandita del suo scudiero.

VII GS: «(ist erschrocken aufgefabren, lauscht und beobachtet)».

VIII GS: «die».

Ix Didascalia in GS; la partitura vi sostituisce «matt».

x GS: «Schau». 
TRISTAN

Meine Herde?

KURWENAL

Herr, das mein' ich!

Dein das Haus,

Hof und Burg.

Das Volk, getreu

dem trauten Herrn,

so gut es konnt',

hat's Haus und Hof ${ }^{\mathrm{XI}}$ gepflegt,

das einst mein Held

zu Erb' und Eigen

an Leut' und Volk verschenkt, als Alles er verließ,

in fremde ${ }^{\mathrm{xII}}$ Land' zu ziehn.

TRISTAN

In welches Land?

KURWENAL

Hei! nach Kornwall;

kühn und wonnig

was sich da Glanzes,

Glückes ${ }^{\mathrm{xIII}}$ und Ehren

Tristan, mein Held, hehr ertrotzt!

TRISTAN

Bin ich in Kornwall?

KURWENAL

Nicht doch: in Kareol.

TRISTAN

Wie kam ich her?

KURWENAL

Hei nun, wie du kamst?

Zu Roß rittest du nicht;

ein Schifflein führte dich her;

doch zu dem Schifflein

hier auf den Schultern

trug ich dich: die sind breit, sie trugen ${ }^{\mathrm{XIV}}$ dich dort zum Strand. Nun bist du daheim zu Land, im echten Land, im Heimat-Land,

\footnotetext{
XI GS: «Herd».

XII GS: «ferne».

XIII GS: «Glückes | GlanZ ».

xIV «die brachten».
}

TRISTANO

I miei greggi?

KURWENAL

Signore, lo credo bene!

Tua la casa,

il cortile, il castello.

Il popolo fedele

all'amato signore,

come meglio poteva,

ha curato casa e podere,

che un giorno il mio eroe

in eredità e possesso

donò alla sua gente ed al suo popolo,

quando tutto lasciò

per andare in terre lontane.

TRISTANO

In quale terra?

KURWENAL

Oh! in Cornovaglia:

ardito e gioioso

quale splendore colà

di fortuna e d'onore

Tristano, il mio eroe, nobilmente si conquistò!

TRISTANO

Sono in Cornovaglia?

KURWENAL

Ma no: in Kareol.

TRISTANO

Come me ne venni qui?

KURWENAL

Ah, dunque! Come venisti?

A cavallo di certo non venisti;

una navicella ti condusse qui:

però alla navicella

su queste spalle

io ti portai:... esse sono larghe:

esse ti portano colà alla spiaggia.

Ora tu sei a casa tua, a casa tua, a terra!

nella tua vera terra

nella terra tua patria; 
auf eigner Weid' und Wonne, im Schein der alten Sonne, darin von Tod und Wunden du selig sollst gesunden. (Er schmiegt sich an Tristans Brust)

TRISTAN (nach einem kleinen Schweigen)

Dünkt dich das, -18

ich weiß es anders,

doch kann ich's dir nicht sagen.

Wo ich erwacht,

weilt' ich nicht;

doch wo ich weilte,

das kann ich dir nicht sagen.

Die Sonne sah ich nicht, noch sah ich Land und ${ }^{\mathrm{xV}}$ Leute:

doch was ich sah,

das kann ich dir nicht sagen.

Ich war -

wo ich von je gewesen,

wohin auf je ich gehe:

im weiten Reich

der Welten Nacht.

Nur ein Wissen nella delizia dei tuoi pascoli, sotto i raggi del vecchio sole: ivi dalla morte e dalle ferite felicemente guarirai. (Si stringe al petto di Tristano)

TRISTANO (dopo un breve silenzio)

Questo a te sembra?

Io la so altrimenti, ma non te la posso dire.

Dov'io mi sono svegliato,...

non mi sono trattenuto;

però dov'io mi son trattenuto

non te lo posso dire.

Io non vidi il sole, né vidi io terra né popolo:

pure quel ch'io vidi

non te lo posso dire.

Io ero

dove sono sempre stato,

dove per sempre tornerò:

nell'ampio reame

della notte dei mondi.

Solo un sapere

18 Wagner stesso ci ha consegnato una definizione vagamente paradossale dell'atto terzo, che si applica perfettamente a quanto Tristan - e noi spettatori con lui - sta per sperimentare: una sorta di 'furia', di 'follia', di 'delirio sinfonico' (secondo un'annotazione di Cosima relativa al settembre 1878). Ossimoro, per dirla con Thomas Grey, che evoca una dialettica affascinante quanto impossibile tra due poli mutuamente esclusivi: tra il paradigma indiscusso del controllo in musica (la 'sinfonia') e la sua negazione, la perdita del controllo (quale appunto la 'furia', la 'follia', il 'delirio'). Il paradosso di un 'delirare sinfonicamente' (conciliazione dell'apparentemente inconciliabile, di 'sinfonia' e 'follia') è appunto al centro di queste famose pagine del Tristan. Nell'eccellente lettura freudiana di Joseph Kerman, «la lunga scena del 'delirio' documenta il tentativo di Tristan di riconquistare il dominio di sé, dragando la propria vita passata e i propri passati sentimenti per raggiungere una nuova sintesi». Un tentativo che avrà successo, realizzando una sorta di conversione del personaggio (vedi nota 19). Soprattutto, la scena sfrutta ai fini della conversione il parallelismo musicale e drammatico di un «ampio doppio ciclo simmetrico»: per due volte consecutive, infatti, Tristan evolve dall'introspezione all' «estatica pregustazione» dell'arrivo di Isolde, attraversando con l'orchestra i quattro episodi ricordo - maledizione - ricaduta - attesa. Un'articolazione quadripartita che (quanto alla musica) indusse nel 1906 Karl Grunsky a leggere nel ciclo una sorta di «saldatura dei quattro movimenti della sinfonia [der symphonischen Viersätzigkeit]». Qui siamo all'apertura del primo ciclo: la memoria di Tristan è sfocata, poi inizia a funzionare riportando a galla la melodia delle strofe di congedo degli amanti della fine dell'atto precedente (nota 13). È poi la volta di altri reperti musicali del passato. Ma, mentre il motivo del Giorno dilaga (es. 11), il ricordo cede il passo a una furibonda tirata contro tutte le immagini del luminoso - «giorno» (Tag), «luce» (Schein, Licht), «fiaccola» (Leuchte), «scintilla» (Zünde): più o meno, le parole di 'un pesce lasciato ad essiccare al sole', per stare ad un irriverente parallelo dello stesso Wagner. La maledizione al 'giorno' sfinisce Tristan, che ricade sul suo giaciglio. Poi però inizia l'attesa («Isolde kommt!»): un «molto vivace» (Sehr lebhaft) solcato da una figura giubilante in orchestra, imparentata con l'es. 16, che tende al cromatismo del Desiderio (es. 1y). Finché l'anelito diventa illusione: l'immagine della nave in procinto di attraccare eccita Tristan («Es naht, es naht») che, sui caldi accordi del Nachtgesang, dal duetto dell'atto secondo, pregusta la liberazione dal mondo con Isolde.

xv GS: «nicht sah ich Land noch». 
dort uns eigen:

colà è nostro:

göttlich ew'ges

il divino eterno

Ur-Vergessen, -

originario oblio!

wie schwand mir seine Ahnung?

Sehnsücht'ge Mahnung,

nenn' ich dich,

die neu dem Licht

des Tags mich zugetrieben?

Was einzig mir geblieben,

ein heiß-inbrünstig Lieben,

aus Todes-Wonne-Grauen

jagt's mich, ${ }^{\mathrm{XvI}}$ das Licht zu schauen,

das trügend hell und golden

noch dir, Isolden, scheint!

Come mi sparve il suo presagio?

Nostalgico ammonimento,

debbo io nominarti,

che nuovamente alla luce

del giorno m'ha spinto?

Quel che unicamente m'era rimasto, un ardente appassionato amore,

dal voluttuoso crepuscolo della morte

mi ha respinto a guardare la luce, che traditrice e chiara e aurea

ancora per te, Isolda, riluce!

(Kurwenal birgt, von Grausen gepackt, sein Haupt.)

(Kurwenal, preso da orrore, nasconde il capo)

TRISTAN (allmählich sich immer mehr aufrichtend)

Isolde noch ${ }^{\mathrm{x} V I I}$

im Reich der Sonne!

Im Tagesschimmer

noch Isolde!

Welches Sehnen,

welches Bangen,

sie zu sehen

welch Verlangen!

Krachend hört' ich

hinter mir

schon des Todes

Tor sich schließen:

weit nun steht es

wieder offen;

der Sonne Strahlen

sprengt' es auf:

TRISTANO (drizzandosi lentamente sempre piì)

Isolda ancora

nel reame del sole!

Nella luce del giorno

ancora Isolda!

Quale brama!

Quale ansia!

Di vederla

quale desiderio!

Con fragore udii

dietro di me

già della morte

la porta chiudersi:

bene ora ella sta

nuovamente aperta,

i raggi del sole

l'hanno forzata;

mit hell erschloss'nen Augen

muß ich der Nacht enttauchen, -

sie zu suchen,

sie zu sehen,

sie zu finden,

in der einzig

zu vergehen,

zu entschwinden

Tristan ist vergönnt.

con occhi aperti alla luce

debbo io risorgere dalla notte,...

per cercarla,

per vederla,

per trovarla

in lei unica

per perdersi

per sparire

Tristano è stato favorito.

XVI GS: «jagt mich's».

xvII A partire dal 1863, Wagner ha ripetutamente messo in chiaro che la sezione della partitura compresa tra l'intonazione di questo verso e quella del verso «von ihr mich scheuchte!» (bb. 350-418) dovrebbe essere omessa dall'esecuzione. 
Weh, nun wächst

bleich und bang

mir des Tages

wilder Drang!

Grell und täuschend

sein Gestirn

weckt zu Trug

und Wahn mir das ${ }^{\mathrm{x} \text { III }}$ Hirn!

Verfluchter Tag

mit deinem Schein!

Wach'st du ewig

meiner Pein?

Brennt sie ewig,

diese Leuchte,

die selbst Nachts

von ihr mich scheuchte!

Ach, Isolde!

Süße! Holde!

Wann - endlich, wann, ach wann

löschest du die Zünde,

(Immer mehr ermattend)

daß sie mein Glück mir künde?

Das Licht, wann löscht es aus?

(Er sinkt erschöpft leise zurück)

Wann wird es Ruh ${ }^{\text {'xIX }}$ im Haus?

KURWENAL (nach großer Erschütterung aus der Niederschlagenheit sich aufraffend) ${ }^{\mathrm{XX}}$

Der einst ich trotzt',

aus Treu' zu dir,

mit dir nach ihr

nun muß ich mich sehnen!

Glaub meinem Wort,

du sollst sie sehen,

hier - und heut -

den Trost kann ich dir geben,

ist sie nur selbst noch am Leben.

TRISTAN (sehr matt)

Noch losch das Licht nicht aus, noch ward's nicht Nacht im Haus.

Isolde lebt und wacht, sie rief mich aus der Nacht.
Ahimè, ora cresce

pallida, assillante

a me del giorno

la selvaggia angoscia;

con luce stridente, ingannevole,

la sua costellazione

sveglia all'inganno

ed all'illusione la mia mente!

Maledetto giorno

con la tua luce!

Veglierai tu sempre

per il mio tormento?

Arderà sempre

questa fiaccola

che, perfino di notte,

mi allontanò da lei?

Ah Isolda!

dolce Isolda!

Quando finalmente

quando, ah! quando,

spengerai tu la fiaccola

(Sempre più spossato)

perch'ella m'annunzi la mia fortuna?

La luce - quando si spegnerà?

(Ricade esausto lentamente)

Quando si farà quiete in questa casa?

KURWENAL (con grande commozione, risollevandosi dal proprio abbattimento)

Colei ch'io un giorno sfidai

per fedeltà verso di te,

con te verso di lei

debbo ora sospirare.

Credi alla mia parola

tu la vedrai

qui, oggi;

questo conforto io ti posso dare,...

pure ch'ella viva ancora.

TRISTANO (molto debolmente)

Ancora la luce non si è spenta, ancora non s'è fatto notte in casa;

Isolda vive e veglia,

ella m'ha richiamato dalla notte.

\footnotetext{
XVIII GS: «mein».

XIX GS: «Nacht».

xx GS: "(heftig ergriffen)».
} 
KURWENAL

Lebt sie denn, so laß dir Hoffnung lachen. Muß Kurwenal dumm dir gelten, heut sollst du ihn nicht schelten.

Wie tot lagst du

seit dem Tag, da Melot, der Verruchte, dir eine Wunde schlug.

Die böse Wunde,

wie sie heilen?

Mir tör'gem Manne

dünkt' es da,

wer einst dir Morolds

Wunde schloß, der heilte leicht die Plagen von Melots Wehr geschlagen.

Die beste Ärztin

bald ich fand;

nach Kornwall hab' ich

ausgesandt:

ein treuer Mann

wohl übers Meer

bringt dir Isolden her.

TRISTAN (außer sich)

Isolde kommt!

Isolde naht! -

(Er ringt gleichsam nach Sprache)

O Treue! hehre, holde Treue!

(Er zieht Kurwenal an sich und umarmt ihn)

Mein Kurwenal,

du trauter Freund,

du Treuer ohne Wanken,

wie soll dir Tristan danken?

Mein Schild, mein Schirm,

in Kampf und Streit

zu Lust und Leid

mir stets bereit:

wen ich gehaßt,

den haßtest du,

wen ich geminnt,

den minntest du.

Dem guten Marke,

dient' ich ihm hold,

wie warst du ihm treuer als Gold!

Mußt' ich verraten
KURWENAL

S'ella vive,

lascia dunque che ti rida la speranza.

Se anche Kurwenal ti deve parere sempliciotto,

oggi non avrai a lagnarti di lui.

Come morto sei giaciuto

dal giorno

in cui Melot, lo scellerato,

ti inferse una ferita.

La cattiva ferita,

come guarirla?

A me uomo semplice

parve allora,

che colei che un giorno a te di Morold

la ferita chiuse,

costei facilmente guarirebbe le piaghe

inferte dall'arma di Melot.

La migliore sanatrice

subito trovai;

in Cornovaglia ho io

inviato:

un uomo fedele

bene attraverso il mare

ti porterà qui Isolda.

TRISTANO (fuori di sé)

Isolda viene!

Isolda s'appressa!

(Come cercando a stento l'espressione)

O fede! Nobile

dolce fede!

(Trae a sé Kurwenal e lo abbraccia)

Mio Kurwenal, amico caro!

O fedele senza vacillare,

come ti deve ringraziare Tristano?

Mio scudo, mio schermo,

in lotta e battaglia,

in piacere e patire,

a me sempre pronto:

colui che io ho odiato

tu hai odiato,

colui che io ho amato

tu hai amato.

Al buon Marke, quand'io fedelmente lo servivo, a lui fosti più fedele dell'oro!

Quand'io dovetti tradire 
den edlen Herrn,

wie betrog'st du ihn da so gern!

Dir nicht eigen,

einzig mein,

mit-leidest du,

wenn ich leide:

nur - was ich leide,

das - kannst du nicht leiden!

Dieß furchtbare Sehnen,

das mich sehrt;

dieß schmachtende Brennen,

das mich zehrt:

wollt' ich dir's nennen,

könntest du's kennen, -

nicht dort ${ }^{\mathrm{xXI}}$ würdest du weilen;

zur Warte müßtest du eilen,

mit allen Sinnen

sehnend von hinnen

nach dorten trachten und spähen,

wo ihre Segel sich blähen;

wo vor den Winden,

mich zu finden,

von der Liebe Drang befeuert,

Isolde zu mir steuert! -

Es naht, es naht

mit mutiger Hast!

Sie weht, sie weht,

die Flagge am Mast.

Das Schiff, das Schiff!

Dort streicht es am Riff!

Sieh'st du es nicht?

(Heftig)

Kurwenal, sieh'st du es nicht?

(Da Kurwenal, um Tristan nicht zu verlassen, zögert, und Tristan in schweigender Spannung nach ihm blickt, ertönt, wie zu Anfang, näher, dann ferner, die klagende Weise des Hirten)

KURWENAL (niedergeschlagen)

Noch ist kein Schiff zu sehn!

TRISTAN (hat mit abnehmender Aufregung gelauscht, und beginnt nun ${ }^{\mathrm{xxII}}$ mit wachsender Schwermut $)^{19}$ il nobile signore,

come volentieri allora tu lo tradisti!

A te non appartieni,

mio solamente,

mi compassioni

se io patisco:

solo quel ch'io patisco

tu non puoi patire!

Questa spaventevole brama

che mi corrode;

questo spasimante ardore

che mi consuma,

se io te lo dicessi,

se tu lo potessi comprendere,

non là tu indugeresti,

ma t'affretteresti in vedetta...

con tutti i sensi

bramoso di qui dentro

al di fuori ti protenderesti e spieresti

là dove le sue vele si gonfiano;

dove, precedendo i venti.

per trovarmi,

infiammata dal fuoco d'amore,

Isolda verso di me naviga!...

Ecco s'appressa! Ecco s'appressa

con baldanzosa fretta!

Sventola, sventola...

la bandiera all'albero.

Il vascello! Il vascello!

Ecco rasenta la scogliera!

Non lo vedi?

(Impetuosamente)

Kurwenal, non lo vedi?

(Mentre Kurwenal per non lasciare Tristano, esita, e questi con silenziosa tensione guarda su di lui, risuona come da principio, vicina, poi piu lontana, l'aria lamentosa del pastore)

KURWENAL (abbattuto)

Nessun vascello si vede ancora!

TRISTANO (il quale ha prestato ascolto con agitazione in via di calmarsi, comincia ora con crescente malinconia)

\footnotetext{
xxI GS: «hier».

xXII GS: «dann».

19 Ad innescare il secondo ciclo del delirio è appunto la delusione dell'attesa: «la lamentosa melodia del pastore» spegne la speranza e fa ripiombare la musica in tonalità minore (il fa dell'alte Weise della cornamusa). Isol-
} 
Muß ich dich so versteh'n, du alte, ernste Weise, mit deiner Klage Klang? -

Durch Abendwehen

drang sie bang, als einst dem Kind

des Vaters Tod verkündet: durch Morgengrauen

bang und bänger, als der Sohn

der Mutter Los vernahm.

Da er mich zeugt' und starb, sie sterbend mich gebar, die alte Weise sehnsuchts-bang zu ihnen wohl auch klagend drang, die einst mich frug, und jetzt mich frägt,
Così debbo io intenderti, antica, austera melodia, col suono del tuo lamento?

Attraverso le arie della sera, angosciosamente ella penetrò, quando un giorno al fanciullo annunziò la morte del padre:... attraverso il crepuscolo del mattino, angosciosa, sempre più angosciosa, quando il figlio apprese la sorte della madre. Poiché egli generandomi morì, ed ella morendo mi partorì,... l'antica melodia, con aria bramosa, bene a loro anche penetrò col lamento; quella stessa che un giorno mi chiese ed ora mi chiede:

segue nota 19

de non giunge ancora, e Tristano ricomincia «con crescente tristezza» il suo esame introspettivo sulle note dell'alte Weise del corno inglese, che aveva varato il primo ciclo ed ora torna a lanciare il secondo. I livelli di tensione, come suggerisce Kerman, sono dosati accuratamente: all'interno di entrambi i cicli, gli episodi "aumentano di emotività» fino alla ricaduta, mentre nelle coppie di passi paralleli il secondo "è sotto ogni aspetto più profondo e più decisivo di quello precedente». A cominciare dal momento del ricordo: che diventa ora (secondo l'analisi illuminante di Grey) una sorta di 'conversazione musicale a tre attori', collocati in tre luoghi differenti dello spazio teatrale: (1) la alte Weise della cornamusa, dietro le quinte; (2) la voce di Tristan, sul palcoscenico; (3) l'orchestra. Dei tre, la prima ad iniziare è l' "antica canzone» del pastore, cui spetta indubbiamente il ruolo più esposto: la sua funzione è infatti quella di 'attivatore' della conversazione. Nella prima fase (Mäßig langsam: "moderatamente lento») la melodia passa dal corno inglese dietro le quinte ai fiati in orchestra e viceversa. Nella seconda fase («Nein! ach nein!»), la alte Weise attiva anche il resto dell'orchestra, che - sollecitata - prende a funzionare come una specie di «cassa di risonanza della memoria» di Tristano. Questa 'cassa' sprigiona attività leitmotivica nella forma di "un affollarsi di motivi passati», che si intrecciano con la melodia stessa in uno sviluppo di densità crescente. Sviluppo che finisce col debordare in una "polifonia da incubo" (nightmarish polyphony), cui si attaglia perfettamente quell'etichetta di 'delirio sinfonico' sulla quale ci siamo soffermati nella nota 18 (con buona pace di Grunsky, che rifiutava recisamente si potesse cogliere qualcosa di "patologico" ed «isterico» in questo secondo ciclo del monologo tristaniano). Questa volta, però, il ricordo non sfocia più nell'esteriore maledizione del 'giorno': Tristan è ritornato in contatto con il suo passato traumatico, e ora è disposto a riconoscere che i veleni del filtro sono stati distillati dalle sue stesse mani ( «ich selbst, ich selbst - ich hab' ihn gebraut!»). Questa consapevolezza è intonata su un gesto che amplifica il cromatismo discendente del motivo del Dolore (es. $1 x$ ), e che viene subito adottato dall'orchestra (motivo della Maledizione dell'amore: Liebesfluchmotiv, es. 26). Se la memoria questa volta scava più a fondo, anche la ricaduta è più drammatica di quella del primo ciclo: Kurwenal teme per la vita di Tristan. Ma anche l'attesa porta a un epilogo diverso: l'unica «guaritrice» (Ärztin), Isolde, sta giungendo davvero (vedi nota 20).

ESEMPIO 26 (bb. 810-11)

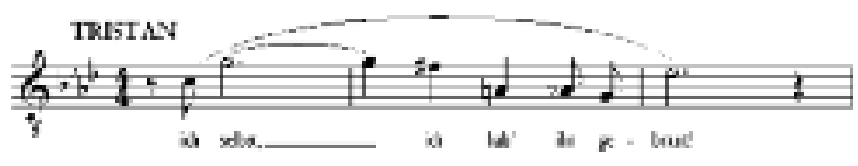


zu welchem Los erkoren

ich damals wohl geboren?

$\mathrm{Zu}$ welchem Los? -

Die alte Weise

sagt mir's wieder: -

mich sehnen - und sterben! ${ }^{\text {xxIII }}$

Nein! ach nein!

So heißt sie nicht:

Sehnen! Sehnen -

im Sterben mich zu sehnen, vor Sehnsucht nicht zu sterben! -

Die nie ${ }^{\mathrm{xxIV}}$ erstirbt,

sehnend nun ruft

um $^{\mathrm{xxv}}$ Sterbens Ruh

sie der fernen Ärztin zu. -

Sterbend lag ich

stumm im Kahn,

der Wunde Gift

dem Herzen nah:

Sehnsucht klagend

klang die Weise;

den Segel blähte der Wind

hin zu Irlands Kind.

Die Wunde, die

sie heilend schloß,

riß mit dem Schwert

sie wieder los;

das Schwert dann aber

ließ sie sinken,

den Gifttrank gab sie

mir zu trinken;

wie ich da hoffte,

ganz zu genesen,

da ward der sehrendste

Zauber erlesen,

daß nie ich sollte sterben, mich ew'ger Qual vererben.

Der Trank! Der Trank!

Der furchtbare Trank!

Wie vom Herzen zum Hirn

er wütend mir drang!

Kein Heil nun kann, a quale sorte destinato

nacqui io allora?

A quale sorte?

L'antica melodia

me lo ripete ancora:

a bramare... e morire!

No! Ah no!

Non così ella vuole!

Bramare! Bramare!

Nel morire bramare,

non di brama morire!

Quella melodia che non muore,

ora chiama bramando,

per la pace del morire

la lontana sanatrice...

Morente io giaceva,

e muto, nella navicella,

col veleno della ferita

presso al cuore;

bramosamente lamentando

risuonò la melodia;

il vento gonfiò la vela

verso la figlia d'Irlanda.

La ferita, che

ella guarendo chiuse,

con la spada sbendò

ella nuovamente;

ma poi la spada...

ella lasciò cadere;

la bevanda avvelenata ella dette

a me da bere:

quand'io sperai

di guarire veramente,

allora fu il più divorante

filtro scelto,

affinché io non avessi mai a morire,

ma ad assoggettarmi ad un tormento eterno.

Il filtro! Il filtro!

Il terribile filtro!

Come dal cuore al cervello

infuriando mi penetrò!

Nessun rimedio ora può,

xxIII GS aggiunge un verso: «sterben - und mich sehnen!».

xxIV GS: «nicht».

xxv GS: «nach». 
kein süßer Tod

je mich befrei'n

von der Sehnsucht Not.

Nirgends, ach nirgends

find' ich Ruh:

mich wirft die Nacht

dem Tage zu,

um ewig an meinen Leiden

der Sonne Auge zu weiden.

O dieser Sonne

sengender Strahl,

wie brennt mir das Hirn ${ }^{\mathrm{x} v 1}$

seine glühende Qual!

Für dieser Hitze

heißes Verschmachten

ach! keines Schattens

kühlend Umnachten!

Für dieser Schmerzen

schreckliche Pein,

welcher Balsam sollte

mir Lind'rung verleih'n?

Den furchtbaren Trank,

der der Qual mich vertraut,

ich selbst, ich selbst -

ich hab' ihn gebraut!

Aus Vaters-Not

und Mutter-Weh,

aus Liebestränen

eh und je,

aus Lachen und Weinen,

Wonnen und Wunden,

hab' ich des Trankes

Gifte gefunden!

Den ich gebrau't, der mir geflossen, den Wonne-schlürfend

je ich genossen, -

verflucht sei, furchtbarer Trank!

Verflucht, wer dich gebraut!

(Er sinkt ohnmächtig zurück)

KURWENAL (der vergebens Tristan zu mäßigen suchte, schreit entsetzt laut auf)

Mein Herre! Tristan! -

Schrecklicher Zauber! - nessuna dolce morte

mai liberarmi

dal tormento della brama;

in nessun luogo, ah! in nessun luogo

troverò pace:

mi rigetta la notte

nuovamente al giorno,

per eternamente del mio patire

gli occhi del sole pascere.

Oh di questo sole

lo struggente raggio,

come mi brucia la fronte

il suo ardente strazio!

Per di questo ardore

l'ardente languire,

ah! di nessun'ombra

[mai] fresco annottare!

Contro di questi tormenti

la terribile pena, quale balsamo potrebbe

mai portarmi sollievo?

Quel terribile filtro,

che m'ha consegnato alla sofferenza,

io stesso... io stesso

l'ho preparato!

Con l'angoscia di mio padre,

col soffrire di mia madre,

con lagrime d'amore,

in ogni tempo,...

con riso e con pianto,

con voluttà e con ferite,

ho del filtro

i veleni trovato!

Quel filtro che ho preparato,

che per me è stato versato,

che sorseggiando voluttà,

io ho un giorno bevuto,...

sia maledetto, terribile filtro!

Maledetto, chi t'ha preparato!

(Ricade svenuto)

KURWENAL (che invano ha cercato di calmare Trista-

no, grida spaventato)

Mio signore! Tristano!

Spaventevole incanto!

xxvı GS: «Herz». 
O Minne-Trug!

O Liebes-Zwang!

Der Welt holdester Wahn, wie ist's um dich getan! -

Hier liegt er nun, der wonnige Mann, der wie Keiner geliebt und geminnt!

Nun seht, was von ihm sie Dankes gewann, was je Minne sich ${ }^{\text {XxVII }}$ gewinnt!

(Mit schluchzender Stimme)

Bist du nun tot?

Lebst du noch?

Hat dich der Fluch entführt? (Er lauscht seinem Atem)

O Wonne! Nein! (zart)

Er regt sich! Er lebt! -

Wie sanft er die Lippen rührt!

TRISTAN (langsam wieder zu sich kommend)

Das Schiff - sieh'st du's noch nicht?

KURWENAL

Das Schiff? Gewiß, es ${ }^{\text {xxvIII }}$ naht noch heut';

es kann nicht lang mehr säumen.

TRISTAN

Und drauf Isolde, wie sie winkt wie sie hold mir Sühne trinkt?

Sieh'st du sie?

Siehst du sie noch nicht?

Wie sie selig,

hehr und milde

wandelt durch

des Meers Gefilde?

Auf wonniger Blumen

lichten ${ }^{\text {XxIx }}$ Wogen

kommt sie sanft ${ }^{\mathrm{xxx}}$

ans Land gezogen:
O inganno dell'ebrezza!

O tirannia dell'amore!

O dolcissima tra le illusioni del mondo, com'è finita per te!

Ecco egli ora giace,

l'uomo gioioso,

che ha amato e s'è inebriato come nessun altro!

Ora vedete, quale da lui

ringraziamento l'amore n'ha colto, che cosa mai ne guadagna!

(Con voce singhiozzante)

Sei dunque morto?

$\mathrm{O}$ vivi ancora?

La maledizione t'ha rapito?

(Ascolta il suo respiro)

O felicità! No!

Egli si muove, egli vive!...

(Con tenerezza)

Come dolcemente muove le labbra!

TRISTANO (lentamente tornando in sé)

Il vascello? Non lo vedi tu ancora?

KURWENAL

Il vascello? Certo

oggi stesso s'appresserà;

non può più tardar oltre.

TRISTANO

E su di esso Isolda,

com'ella accenna,...

come dolce

ella beve a me il perdono:

la vedi?

Non la vedi ancora?

Come beata,

e nobile e dolce,

si aggira per

i campi del mare?

Su di fiori deliziosi

onde luminose,

viene ella dolcemente

tratta a terra.

\footnotetext{
xxvII GS: «je sich Minne».

xxVIII GS: «das».

xxIX GS: «sanften».

xxx GS: «licht».
} 
sie lächelt mir Trost

und süße Ruh;

sie führt mir letzte

Labung zu.

Isolde! Ach, Isolde, wie schön bist du! - ${ }^{\mathrm{xxx}}$

Und Kurwenal, wie?

Du sähst sie nicht?

Hinauf zur Warte,

du blöder Wicht,

was so hell und licht ich sehe,

daß das dir nicht entgehe.

Hörst du mich nicht?

Zur Warte schnell!

Eilig zur Warte!

Bist du zur Stell'?

Das Schiff, das Schiff!

Isoldens Schiff -

du mußt es sehen,

mußt es sehen!

Das Schiff - säh'st du's noch nicht?

(Während Kurwenal noch zögernd mit Tristan ringt, läßt der Hirt von außen die Schalmei ertönen $\left.{ }^{\mathrm{xx} \times I}\right)$

KURWENAL (freudig aufspringend und der Warte zueilend)

O Wonne! Freude!20

Ha! Das Schiff!

Von Norden seh' ich's nahn.

TRISTAN (mit wachsender Begeisterung)

Wußt' ich's nicht?

Sagt' ich es nicht?

Daßs sie noch lebt,

noch Leben mir webt?

Die mir Isolde

einzig enthält,

wie wär' Isolde

mir aus der Welt?
Ella mi sorride conforto

e dolce riposo.

Porta ella a me l'ultimo

conforto.

Ah! Isolda, Isolda!

Come sei bella!

Kurwenal, come,

non la vedesti?

Su in vedetta,

uomo dalla corta vista!

Quel che così chiaro e luminoso io vedo,

che non ti sfugga.

Non mi odi?

Presto in vedetta!

Subito in vedetta!

Sei al tuo posto?

Il vascello! Il vascello!

Il vascello d'Isolda?

Lo devi vedere!

Lo devi vedere!

Il vascello? Non lo vedesti ancora?

(Mentre Kurwenal esitando lotta ancora con Tristano, il pastore dal di fuori fa risuonare la zampogna) KURWENAL (balzando in piedi con gioia e salendo precipitosamente al posto di vedetta)

$\mathrm{O}$ voluttà! $\mathrm{O}$ gioia!

Ah! Il vascello!

Lo vedo appressarsi dal nord.

TRISTANO (con crescente esaltazione)

Non lo sapevo forse?

Non lo dissi forse?

Che ella ancora vive, ed ancora per me tesse la vita?

Quel mondo che per me Isolda solo contiene,

come sarebbe Isolda

fuori di quel mondo?

xxxI GS: «wie hold, wie schön bist du! -».

xxxII GS: «einen lustigen Reigen vernehmen».

20 Una elementare melodia in Do del corno inglese (ma Wagner suggerisce la fabbricazione di un apposito Alpenhorn: vedi appendice alla guida) suggerisce l'avvistamento della nave di Isolde. L'orchestra si unisce al giubilo con una pagina Sehr lebhaft («molto vivace»), in cui si giova anche della melodia di «Barg im Busen» (vedi nota 12), appena reintrodotta nel tessuto leitmotivico. 
KURWENAL (von der Warte zurückrufend, jauchzend)

Hahei! Heiha! ${ }^{\text {xxxIII }}$

Wie es mutig steuert!

Wie stark der ${ }^{\text {xxiv }}$ Segel sich bläht!

Wie es jagt! Wie es fliegt!

TRISTAN

Die Flagge? Die Flagge?

KURWENAL

Der Freude Flagge

am Wimpel lustig und hell.

TRISTAN (auf dem Lager hoch sich aufrichtend)

Hahei ${ }^{\mathrm{xxx}}$ der Freude!

Hell am Tage

zu mir Isolde,

Isolde zu mir! -

Siehst du sie selbst?

KURWENAL

Jetzt schwand das Schiff

hinter dem Fels.

TRISTAN

Hinter dem Riff?

Bringt es Gefahr?

Dort wütet die Brandung,

scheitern die Schiffe. -

Das Steuer, wer führt's?

KURWENAL

Der sicherste Seemann.

TRISTAN

Verriet' er mich?

Wär' er Melots Genoß?

KURWENAL

Trau ihm wie mir!

TRISTAN

Verräther auch du! -

Un-seliger!

Siehst du sie wieder?

KURWENAL

Noch nicht.

TRISTAN

Verloren!
KURWENAL (volgendosi a chiamare dal posto di vedetta, giubilante)

Hahei! Heiha!

Come naviga baldanzosamente!

Con quanta forza si gonfia la vela!

Come corre! Come vola!

TRISTANO

La bandiera? La bandiera?

KURWENAL

Bandiera di gioia, pressi il gagliardetto, gaia e luminosa.

TRISTANO (drizzandosi alto sul giaciglio)

Evviva! Quale gioia!

Luminosa nel giorno

a me Isolda!

Isolda a me!

La vedi tu in persona?

KURWENAL

Ora il vascello è scomparso

dietro la rupe.

TRISTANO

Dietro la scogliera?

Porterà pericolo?

Là la risacca infuria,

e naufragano i vascelli!

Il timone chi lo tiene?

KURWENAL

Il pilota più sicuro.

TRISTANO

Mi vorrebbe tradire?

Sarebbe compagno di Melot?

KURWENAL

Di lui fidati come di me!

TRISTANO

Traditore anche te!

Sciagurato!

La vedi nuovamente?

KURWENAL

Non ancora.

TRISTANO

Perduta!

\footnotetext{
xxxIII GS: «Hahei!».

xxxIv GS: «das».

xxxv GS: «Heiaha!».
} 


\author{
KURWENAL (jauchzend) \\ Haha! Heiahaha! \\ Vorbei! Vorbei! \\ Glücklich vorbei! ${ }^{\mathrm{XxxvI}}$ \\ TRISTAN (jauchzend) \\ Heiaha! Kurwenal! \\ Treuester Freund! \\ All' mein Hab und Gut \\ Vererb' ich noch heute. ${ }^{\mathrm{xxxvII}}$ \\ KURWENAL \\ Sie nahen im Flug. \\ TRISTAN \\ Sieh'st du sie endlich? \\ Sieh'st du Isolde? \\ KURWENAL \\ Sie ist's! Sie winkt! \\ TRISTAN \\ O seligstes Weib! \\ KURWENAL \\ Im Hafen der Kiel! - \\ Isolde - ha! \\ mit einem Sprung \\ springt sie vom Bord ans Land. ${ }^{\mathrm{XxVIII}}$ \\ TRISTAN \\ Herab von der Warte! \\ Müßiger Gaffer! \\ Hinab! Hinab \\ an den Strand! \\ Hilf ihr! Hilf meiner Frau! \\ KURWENAL \\ Sie trag' ich herauf: \\ trau' meinen Armen! \\ Doch du, Tristan, \\ bleib mir treulich am Bett! \\ (Er eilt durch das Tor hinab.)
}

KURWENAL (giubilando)

Evviva! Hei ha ha ha!

Passato, passato!

Felicemente passato!

TRISTANO (giubilando)

Evviva! ha ha ha! Kurwenal, fedelissimo amico!

Ogni mio possesso ed avere

oggi stesso io ti lego.

KURWENAL

Si avvicinano di volo.

TRISTANO

La vedi finalmente?

Vedi tu Isolda?

KURWENAL

È lei! Ella accenna!

TRISTANO

Donna celeste!

KURWENAL

Nel porto il vascello!

Isolda, ah!

con un salto

salta da bordo a terra.

TRISTANO

Giù dalla vedetta,

ozioso badalucco!

Giù! Giù

alla spiaggia!

Aiutala! Aiuta la mia donna!

KURWENAL

La porterò io quassù:

fidati delle mie braccia!

ma tu, Tristano,

fedele al mio consiglio, rimani sul tuo letto!

(Kurwenal esce rapidamente)

xxxvI GS aggiunge: «Im sich'ren Strom I steuert zum Hafen das Schiff».

xxxvII GS: «erbst du noch heut».

xxxVIII GS: «zum Strand». 


\section{ZWEITE SZENE}

TRISTAN (in höchster Aufregung auf dem Lager sich mühend)

$0^{\text {xxxIx }}$ diese Sonne! ${ }^{21}$

$\mathrm{Ha}$, dieser Tag!

$\mathrm{Ha}$, dieser Wonne

sonnigster Tag!

Jagendes Blut, jauchzender Mut!

Lust ohne Maßen, freudiges Rasen:

auf des Lagers Bann

wie sie ertragen?

Wohlauf und daran, wo die Herzen schlagen!

Tristan, der Held, in jubelnder Kraft

hat sich vom Tod emporgerafft!

(Er richtet sich hoch auf)

Mit blutender Wunde

bekämpft' ich einst Morolden:

mit blutender Wunde

erjag' ich mir heut Isolden.

(Er reißt sich den Verband der Wunde auf)

$\mathrm{Heia}^{\mathrm{XL}}$ mein Blut,

lustig nun fließe!

(Er springt vom Lager herab und schwankt vorwärts)

Die mir die Wunde

ewig $^{\mathrm{xLI}}$ schließe,

sie naht wie ein Held,

sie naht mir zum Heil:

\section{SCENA SECONDA}

TRISTANO (nella massima agitazione, dimenandosi sul suo giaciglio)

Oh! questo sole!

Ah! questo giorno!

Ah! di questa voluttà

radiosissimo giorno!

Sangue in tumulto!

Anima giubilante!

Letizia senza misura, gioioso delirio!

Nei vincoli del giaciglio

come sopportarli?

Orsù, in piedi,

dove battono i cuori!

Tristano, l'eroe,

con la forza della gioia,

si è dalla morte

su svincolato!

(Si drizza di tutta la persona)

Con sanguinosa ferita

io mi battei un giorno contro Morold:

con sanguinosa ferita

oggi io conquisto Isolda!

(Si strappa le bende dalla ferita)

Evviva, il mio sangue!

Ch'esso scorra in letizia!

(Balza dal suo giaciglio e s'avanza barcollando)

Colei che la mia ferita

avrà a chiudere per sempre,...

ella s'appressa come un eroe,

ella s'appressa per la mia salute!

xxxIx GS: «Ha,».

21 È sempre la melodia di «Barg im Busen», che pulsa sincopata, irrequieta e molto crescendo nei bassi dell'orchestra, a esprimere l'insofferenza di Tristan che si agita sul suo letto, impaziente di correre incontro alla sua 'guaritrice'. E una pagina allucinata e originalissima: ad un certo punto la frenesia ritmica s'impadronisce del placido motivo del Sopore (es. 17x), stravolgendolo nel fortissimo di una versione sghemba e brutale (Wagner lo riscrive nel tempo assai insolito di $\mathbf{4}_{\mathbf{4}}^{\mathbf{5}}$. Nel momento culminante, quando l'uomo si precipita barcollando tra le braccia di Isolde, il complesso Dolore-Desiderio (i due temi del preludio: ess. 1-2) riconquista prepotentemente la scena musicale, in modo analogo a quanto era accaduto alla fine dell'atto primo, dopo la libagione del filtro (vedi nota 7). Il parallelismo ovviamente non è casuale. Su un tempo in costante dilatazione (un allargando), Tristan si spegne sulla prima frase del motivo dello Sguardo $(2 y)$ e il filo melodico viene reciso: i violoncelli lasciano la frase incongruamente sospesa sul $\mathrm{Sib}_{3}$ - un attimo prima dello «Ha!» di Isolde.

XL. GS: «Hahei!».

XLI GS: «auf ewig». 
vergehe die Welt

meiner jauchzenden Eil'!

(Er taumelt nach der Mitte der Bühne) $)^{\mathrm{XLII}}$

ISOLDE (von außen rufend)

Tristan! Geliebter!

TRISTAN (in der furchtbarsten Aufregung)

Wie, hör' ich das Licht?

Die Leuchte - ha!

Die Leuchte verlischt!

$\mathrm{Zu}$ ihr! Zu ihr!

(Isolde eilt atemlos herein. Tristan, seiner nicht mächtig, stürzt sich ihr schwankend entgegen. ${ }^{\text {XLIII In }}$ der Mitte der Bühne begegnen sie sich; sie empfängt ihn in ihren Armen. Tristan sinkt langsam in ihren Armen zu Boden)

ISOLDE

Tristan! Ha!

TRISTAN (sterbend zu ihr aufblickend) ${ }^{\text {XIV }}$ Isolde! -

(Er stirbt) $^{\mathrm{xLV}}$

ISOLDE

Ha! ${ }^{\text {XVI }}$ Ich bin's, ich bin's - 22

süßester Freund!

Auf! noch einmal!

Hör meinen Ruf! ${ }^{\mathrm{XuVII}}$

Isolde ruft:

Isolde kam,

mit Tristan treu zu sterben. -

Bleibst du mir stumm?

Nur eine Stunde, -

nur eine Stunde

bleibe mir wach!

So bange Tage
Che il mondo si sperda

di fronte alla mia fretta giubilante!

(S'avanza barcollando verso il centro della scena)

ISOLDA (dal di fuori)

Tristano! caro!

TRISTANO (nella più tremenda esaltazione)

Come, odo io la luce?

La fiaccola, ah!

La fiaccola si spenge!

A lei! A lei!

(Isolda entra in fretta senza più respiro. Tristano, non potendosi più sostenere, le si precipita incontro barcollando. Nel mezzo della scena si incontrano: ella lo riceve tra le sue braccia. Tristano, nelle stesse braccia di lei, cade lentamente al suolo)

ISOLDA

Tristano! Ah!

TRISTANO (morendo con lo sguardo fisso su di lei) Isolda!

(Muore)

ISOLDA

Ah! sono io, sono io, dolcissimo amico!

$\mathrm{Su}$, ancora una volta

odi il mio grido!

Isolda chiama:

Isolda è venuta

a morire fedelmente con Tristano!

Mi resti muto?

Un'ora sola,

un'ora sola,

restami sveglio!

Così angosciosi giorni

\footnotetext{
XLII GS: «(Er hat sich ganz aufgerafft, und springt jetzt vom Lager)».

XLII GS: "Er stürzt taumelnd der hereineilenden Isolde entgegen».

XLIV GS: «(in Isoldes Arm sinkend)».

XIV GS: «(Den Blick zu ihr aufgeheftet, sinkt er leblos in ihren Armen langsam zu Boden)».

XIVI GS: «(nach einem Schrei)».

xLVII In GS segue il verso: «Achtest du nicht?».

22 Il lamento di Isolde è articolato da un refrain basato sul cosiddetto motivo del Dubbio di morte (Motiv des Todesfrage: es. 27): una serie di sincopi angosciose e dissonanti (nell'oboe) che si scaricano in una linea melodica inquieta almeno quanto il secondo motivo della Morte (es. 17y), con il quale il Dubbio di morte condivide la sonorità del Tristanakkord $\left(\mathrm{Re}^{2}-\mathrm{Lab}_{2}-\mathrm{Do}_{3}-\mathrm{Fa}_{3}\right)$ e la clausola melodica ( $x$ nell'es. 27) nella forma del motivo del Fato (es. $2 x$ ).
} 
wachte sie sehnend,

um eine Stunde

mit dir noch zu wachen.

Betrügt Isolden,

betrügt sie Tristan

um dieses einz'ge

ewig-kurze

letzte Welten-Glück? -

Die Wunde - wo?

Laß sie mich heilen,

daß wonnig und hehr

die Nacht wir teilen.

Nicht an der Wunde,

an der Wunde stirb mir nicht! ella ha vegliato struggendosi,

per un'ora sola

ancora vegliare con te:

tradisce Isolda,

la tradisce Tristano,

di questa unica

breve ed eterna

estrema felicità del mondo?

La ferita? Dove?

$\mathrm{Fa}$ ' che io la guarisca!

Che in sublime letizia

partecipiamo alla notte;

non della ferita

non morirmi della ferita:

segue nota 22

ESEMPIO 27 (bb. 1334-1338)

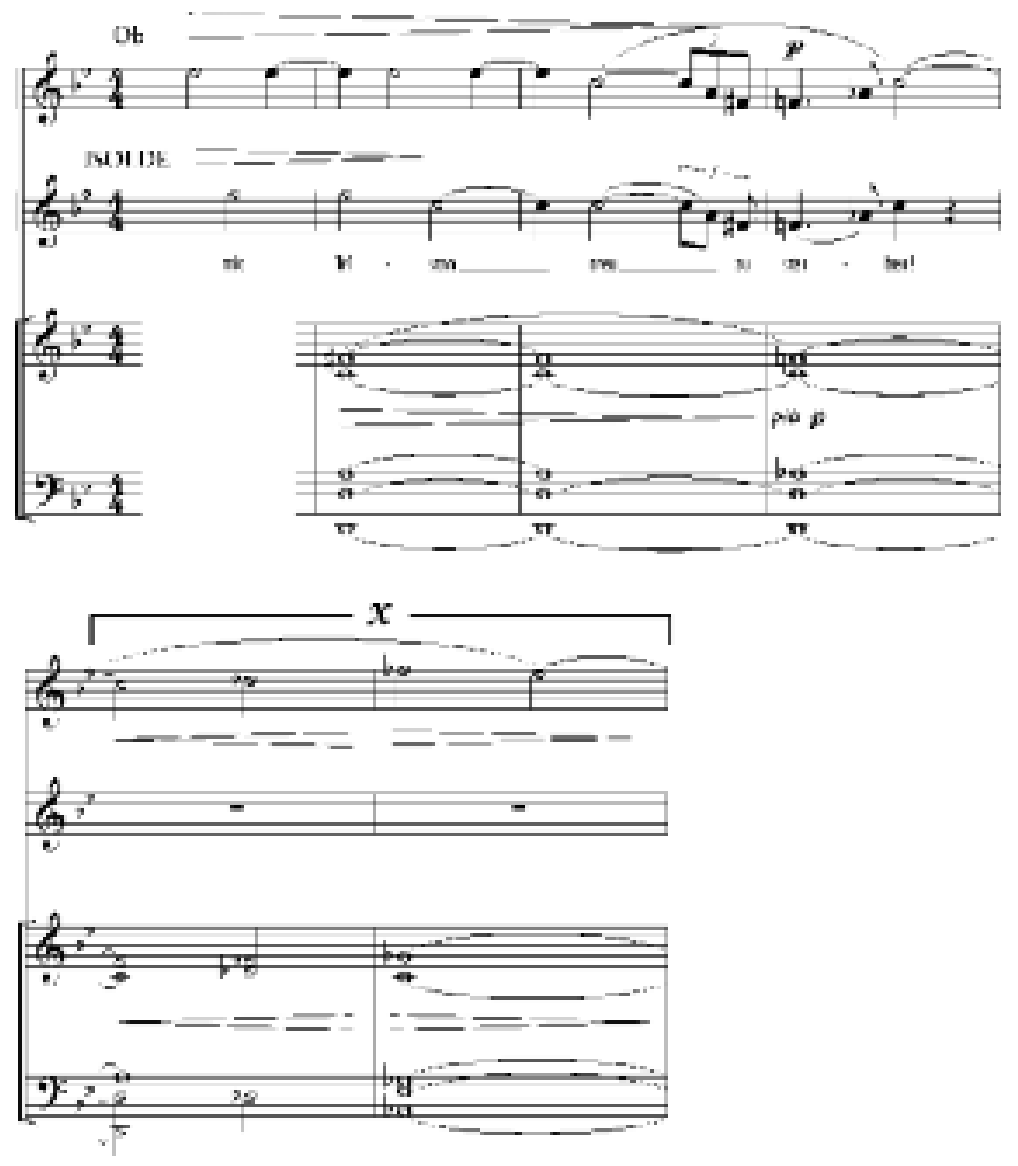


Uns beiden vereint erlösche das Lebenslicht! -

Gebrochen der Blick! -

Still das Herz! -- xuvIII

Nicht eines Atems

flücht'ges Wehn?

Muß sie nun jammernd

vor dir stehn,

die sich wonnig dir zu vermählen

mutig kam über Meer?

Zu spät! ${ }^{\mathrm{XLIX}}$

Trotziger Mann!

Strafst du mich so

mit härtestem Bann?

Ganz ohne Huld

meiner Leidens-Schuld?

Nicht meine Klagen

darf ich dir sagen?

Nur einmal, ach!

Nur einmal noch! -

Tristan - ha!

horch - er wacht!

Geliebter - ${ }^{\mathrm{L}}$

(Sie sinkt bewußtlos ${ }^{\mathrm{LI}}$ über der Leiche zusammen)

\section{DRITTE SZENE}

(Kurwenal war sogleich hinter Isolde zurückgekommen; sprachlos in furchtbarer Erschütterung hat er dem Auftritte beigewohnt, und bewegungslos auf Tristan hingestarrt. Aus der Tiefe hört man jetzt dumpfes Getümmel und Waffengeklirr. - Der Hirt kommt über die Mauer gestiegen, hastig und leise zu Kurwenal sich wendend) 23

HIRT

Kurwenal! Hör'!

Ein zweites Schiff. che ad ambedue congiunti

si spenga la luce della vita!

Spento lo sguardo!

Fermo il cuore!

Non di un respiro

il soffio fuggitivo!...

Deve colei dunque in dolore

stare a te davanti,

che per la gioia di congiungersi a te

attraversò coraggiosamente il mare?

Troppo tardi!

Uomo sdegnoso!

Così dunque tu mi punisci

col più duro dei decreti?

Senza alcuna pietà

per la colpa del mio soffrire?

I miei lamenti

non posso io dire?

Una volta sola ah!

una volta sola ancora!...

Tristano!... Ah!...

Odi! Si sveglia!

Caro!

(Ella s'abbatte svenuta sul cadavere)

\section{SCENA TERZA}

(Kurwenal, che è rientrato subito dietro Isolda, ha assistito alla scena senza far motto in spaventevole commozione ed immobile, gli occhi fissi su Tristano. Dal basso s'ode ora un sordo tumulto ed uno strepito d'armi. Il pastore entra scavalcando il parapetto, volgendosi prontamente e sommesso a Kurwenal)

\section{IL PASTORE}

Kurwenal! Odi!

Un altro vascello.

\footnotetext{
XıVIII In GS seguono due versi supplementari: «Treuloser Tristan, I mir diesen Schmerz?».

xuIx GS: «Zu spät! | Zu spät!».

${ }^{L}$ GS: segue il verso «- Nacht!».

L. GS: «ohnmächtig».

23 Tremoli e veloci scalette degli archi ci introducono all'ecatombe che si consuma rapidamente sulla scena: Isolde è esanime sul cadavere di Tristan, quando cadono Melot e Kurwenal. L'orchestra insiste particolarmente sulla figura di giubilo imparentata col Desiderio (es. $1 y$ ) che aveva suggellato l'attesa delirante di Tristan (vedi nota 18): quasi a sottolineare ironicamente il potenziale tragico della brama, della «volontà di vivere [Wille zum
} 
(Kurwenal fährt auf und blickt über Brüstung, während der Hirt aus der Ferne erschüttert auf Tristan und Isolde sieht)

KURWENAL

Tod und Hölle!

(In Wut ausbrechend) ${ }^{\text {LII }}$

Alles zur Hand!

Marke und Melot

hab' ich erkannt. -

Waffen und Steine!

Hilf mir! Ans Tor!

(Er springt mit dem Hirt an das Tor, das Beide in der Hast zu verrammeln suchen)

DER STEUERMANN (stürat herein)

Marke mir nach

mit Mann und Volk!

Vergebne Wehr!

Bewältigt sind wir.

KURWENAL

Stell dich, und hilf! -

So lang ich lebe,

lugt mir keiner herein!

BRANGÄNES STIMME (außen, von unten her)

Isolde, Herrin!

KURWENAL

Brangänens Ruf?

(Hinabrufend)

Was suchst du hier?

BRANGÄNE

Schließ nicht, Kurwenal!

Wo ist Isolde?

KURWENAL

Verrät'rin auch du?

Weh dir, Verruchte!
(Kurwenal trasalisce con violenza ed osserva al di là del parapetto, mentre il pastore guarda di lontano, commosso, Tristano e Isolda)

KURWENAL

Morte e inferno!

(Rompendo in furore)

Che tutto sia pronto!

Marke e Melot

ho riconosciuto.

Armi e pietre!

Aiuto! Alla porta!

(Si affretta col pastore alla porta, che in fretta cercano di sprangare)

IL PILOTA (si precipita dentro)

Marke mi segue

con la sua masnada:

inutile difesa!

Siamo sopraffatti.

KURWENAL

Mettiti alla difesa ed aiutami!

Per quanto vivrò,

nessuno mi metterà piede qui dentro!

LA VOCE DI BRANGANIA (fuori, dal basso)

Isolda! Signora!

KURWENAL

Il grido di Brangania?

(Gridando verso il basso)

Che cerchi tu qui?

BRANGANIA

Non chiudere, Kurwenal!

Dov'è Isolda?

KURWENAL

Traditrice anche tu?

Guai a te, infame!

segue nota 23

Leben]» schopenhaueriana. Come prima Isolde, tocca ora a Marke intonare la sua - e la nostra - costernazione ( «O Trug und Wahn!») sulle note in orchestra del Dubbio di morte (es. 27), poi sul suo motivo dell'atto secondo (es. 21: «Trautester Freund!»). Incastonata nel cordoglio di Marke, la voce di Brangäne ci regala una delle sua pagine più intense («Sie wacht! Sie lebt!»), che si inerpica esitante sulle note del motivo del Desiderio (es. 1y) fino a un indimenticabile $\mathrm{Lab}_{4}$ («dir zu ent-sa-gen»). I gemiti del re e dell'ancella interrompono ripetutamente il 'canto di morte' dal tempo lento della scena d'amore (vedi nota 12 e l'es. 18), già rimesso in gioco dal pianto di Isolde (da «daß wonnig und hehr»: vedi nota 22): violini e corni con sordina lo rilanciano piano per tre volte, in tre tonalità che disegnano una lenta ascesa cromatica (Fa-Solb-Solb).

LII GS: «KURWENAL (in Wut ausbrechend) I Tod und Hölle!». 
MELOTS STIMME (von außen)

Zurück, du Tor!

Stemm dich nicht dort! ${ }^{\mathrm{LIII}}$

KURWENAL (wütend auflachend)

Heiahaha! $!^{\text {LV }}$ dem Tag,

an dem $^{\mathrm{LV}}$ ich dich treffe!

(Melot, mit gewaffneten Männern, erscheint unter dem Tor. Kurwenal stürat sich auf ihn und streckt ihn zu Boden)

Stirb, schändlicher Wicht!

MELOT (sterbend)

Wehe mir! - Tristan!

BRANGÄNE (immer noch außen)

Kurwenal! Wütender!

Hör, du betrügst dich.

KURWENAL

Treulose Magd! -

(Zu den Seinen)

Drauf! Mir nach!

Werft sie zurück!

(Sie kämpfen)

MARKE (von außen)

Halte, Rasender!

Bist du von Sinnen?

KURWENAL

Hier wütet der Tod!

Nichts andres, König,

ist hier zu holen:

willst du ihn kiesen, so komm!

(Er dringt auf Marke und dessen Gefolge ${ }^{\mathrm{LVI}}$ ein)

MARKE (unter dem Tore mit Gefolge erscheinend)

Zurück, Wahnsinniger!

BRANGÄNE (hat sich seitwärts über die Mauer geschwungen und eilt in den Vordergrund)

Isolde! Herrin!

Glück und Heil! -

Was seh' ich, ha!

Lebst du? Isolde!

(Sie stürzt auf Isolde, und müht sich um sie. Während dem hat Marke mit seinem Gefolge
LA VOCE DI MELOT (dal difuori)

Vattene pazzo!

Non ti opporre costà!

KURWENAL (ridendo rabbiosamente)

Evviva il giorno, in cui t'incontro!

(Melot con scorta armata appare sulla soglia. Kurwenal si precipita su di lui e lo stende al suolo)

Muori, ignobile furfante!

MELOT (morendo)

Guai a me! Tristano!

BRANGANIA (ancora dal di fuori)

Kurwenal! Furioso!

Ascolta, t'inganni!

KURWENAL

Serva infedele!

(Ai suoi)

Avanti! Seguitemi!

Ricacciateli!

(Combattono)

MARKE (dal di fuori)

Fermati, furibondo!

Sei fuori di te?

KURWENAL

Qui infuria la morte!

Nient'altro, o re,

c'è qui da prendere:

se tu la vuoi, avanzati!

(Si precipita su Marke e sul suo seguito)

MARKE (comparendo sulla soglia col seguito)

Indietro! Folle!

BRANGANIA (girando, ha saltato il parapetto, e s'affretta sul davanti della scena)

Isolda! Signora!

Fortuna! Salvezza!

Che vedo? Ah!

Vivi? Isolda!

(Si dà premura intorno ad Isolda. - Marke col suo seguito, avendo respinto Kurwenal ed $i$ suoi compagni

\footnotetext{
LIII GS: «dort nicht!».

LIV GS: «KURWENAL | Heiaha».

LV GS: «da».

LVI GS: «ibn».
} 
Kurwenal mit dessen Helfern zurückgetrieben, und dringt berein. Kurwenal, schwer verwundet, schwankt vor ihm her nach dem Vordergrunde)

MARKE

O Trug und Wahn!

Tristan, wo bist du?

KURWENAL

Da liegt er -

hier - wo ich - liege. ${ }^{\text {LVII }}$

(Er sinkt bei Tristans Füßen zusammen)

MARKE

Tristan! Tristan!

Isolde! Weh!

KURWENAL (nach Tristans Hand fassend)

Tristan! Trauter!

Schilt mich nicht,

daß der Treue auch mit kommt!

(Er stirbt)

MARKE

Tot denn Alles!

Alles tot?

Mein Held! Mein Tristan!

Trautester Freund!

Auch heute noch

mußt du den Freund verrathen?

Heut, wo er kommt

dir höchste Treu' zu bewähren?

Erwache! Erwache!

Erwache meinem Jammer,

(Schluchzend über die Leiche sich herabbeugend)

du treulos treuster Freund!

BRANGÄNE (die in ibren Armen Isolde wieder zu sich gebracht)

Sie wacht! Sie lebt!

Isolde! Hör mich,

vernimm meine Sühne! $!^{\mathrm{LVIII}}$

des Trankes Geheimnis

entdeckt' ich dem König:

mit $^{\mathrm{LIX}}$ sorgender Eil'

stach er in See, ${ }^{\mathrm{LX}}$ dalla porta, irrompe. Kurwenal, gravemente ferito, retrocede barcollando davanti a lui, sul davanti della scena)

MARKE

O inganno e delirio!

Tristano! Dove sei?

KURWENAL

Qui egli giace...

qui... dove io... giaccio.

(S'abbatte ai piedi di Tristano)

MARKE

Tristano! Tristano!

Isolda! Guai!

KURWENAL (prendendo la mano di Tristano)

Tristano! Caro!

Non t'avere a male, che anche il tuo fedele venga con te!

(Muore)

MARKE

Tutti morti, dunque!

Tutti morti!

Mio eroe, mio Tristano!

Caro fra tutti gli amici, anche oggi

devi tradire il tuo amico?

Oggi, mentr'egli viene

a provarti la sua più alta fede?

Svegliati! Svegliati!

Svegliati al mio lamento!

(Curvandosi sul cadavere e singhiozzando)

O fedelissimo infedele amico!

BRANGANIA (che ha preso nuovamente Isolda tra le sue braccia)

È sveglia! Vive!

Isolda! Ascoltami, apprendi la mia espiazione!

Il segreto del filtro

ho scoperto al re:

con impaziente fretta

s'è posto in mare

LVII GS: «Da liegt er - da - | hier, wo ich liege -!».

LVIII GS: «Isolde, hör! Hör mich, süßeste Frau! | Glückliche Kunde I laß mich dir melden: I vertrautest du nicht Brangänen? I Ihre blinde Schuld I hat sie gesühnt; I als du verschwunden, I schnell fand sie den König:».

LIX GS: «erfuhr der kaum, I als mit ».

LX GS: «in See er stach,». 
dich zu erreichen,

dir zu entsagen,

dich zuzuführen den ${ }^{\text {LxI }}$ Freund.

MARKE

Warum, Isolde,

warum mir das?

Da hell mir enthüllt, ${ }^{\text {LXII }}$

was zuvor ich nicht fassen konnt',

wie selig, daß ich den Freund

frei von Schuld da fand!

Dem holden Mann,

dich zu vermählen,

mit vollen Segeln

flog ich dir nach:

doch Unglückes

Ungestüm,

wie erreicht es, wer Frieden bringt?

Die Ernte mehrt' ich dem Tod:

der Wahn häufte die Not!

BRANGÄNE

Hörst du uns nicht?

Isolde! Traute!

Vernimmst du die Treue nicht?

ISOLDE (die nichts um sich her vernommen, heftet das Auge mit wachsender Begeisterung auf Tristans Lei(he) ${ }^{\mathrm{LXIII}}$

Mild und leise ${ }^{24}$

wie er lächelt,

wie das Auge

\author{
per raggiungerti, \\ per rinunziare a te, \\ per condurti l'amico. \\ Perché Isolda, \\ perché a me questo?
}

MARKE

Poiché chiaro mi fu scoperto

quel ch'io dapprima non potevo capire,

come fui felice, che l'amico

libero trovassi d'ogni colpa!

Al valoroso

per sposarti,

a vele gonfie,

io volai dietro di te.

Ma della sventura

l'impeto

come può mai raggiungere colui che porta la pace?

La raccolta io crebbi alla morte:

l'illusione accumulò le sventure!

BRANGANIA

Non ci ascolti?

Isolda! Cara!

Non odi tu la tua fedele?

ISOLDA (che non ha compreso niente intorno a sé, figge lo sguardo con crescente esaltazione sul cadavere di Tristano)

Lieve, sommesso

come sorride,

come l'occhio

LXI GS: «dem».

LXII GS: "ward enthüllt».

LXIII GS: "(die theilnahmlos vor sich hingeblickt, ohne zu vernehmen, heftet das Auge endlich auf Tristan)».

24 L'epilogo del Tristan und Isolde è familiare ai frequentatori delle sale da concerto quale seconda metà del brano universalmente noto come Vorspiel und Isoldens Liebestod («Preludio e morte d'amore di Isolde»). La paternità dell'abbinamento tra il preludio all'atto primo e la morte di Isolde è di Wagner stesso, e l'idea è documentata già nel 1862 (il brano composito fu diretto per la prima volta dal compositore l'anno seguente). Non è invece di stretto conio wagneriano il titolo, che è fonte di qualche confusione. È vero che il termine Liebes-Tod compare in uno dei versi della scena d'amore dell'atto secondo ( «sehnend verlangter I Liebes-Tod!»: "0 ardentemente invocata I morte d'amore!»; vedi nota 13), ma - a ingarbugliare le cose - Wagner lo adopera sempre per riferirsi proprio al preludio dell'atto primo, e non alla morte di Isolde (in altre parole, il Liebestod per lui coincide con il Vorspiel - idea spiazzante che dischiude interessanti prospettive di riflessione). Mentre il titolo riservato da Wagner alla sezione conclusiva del Tristan è Verklärung («trasfigurazione»: per inciso, il responsabile della trasformazione della Verklärung in Liebestod fu nientemeno che il suocero di Wagner, Franz Liszt, che nel 1867 intitolò appunto Isolden's Liebes-Tod la sua fortunata riduzione del monologo finale). Abbiamo dunque a che fare con la trasfigurazione di Isolde, e non con la 'morte d'amore'. Una Verklärung che riprende il filo del 'canto di morte' (Sterbelied: es. 18), interrotto a più riprese nei minuti precedenti (vedi nota 23): e finalmente l'ascesa cromatica Fa-Solb-Solł trova il suo compimento nel Lab di «Mild und leise», che ci fa innanzitutto udire lo Sterbelied 
hold er öffnet:

seht ihr's, Freunde,

säht ihr's nicht?

Immer lichter

wie er leuchtet,

Stern-umstrahlet

hoch sich hebt?

Seht ihr's nicht? ${ }^{\text {IXIV }}$

Wie das Herz ihm

mutig schwillt,

voll und hehr

im Busen ihm quillt;

wie den Lippen

wonnig mild dolce egli apre,...

lo vedete amici?

Non lo vedete?

Sempre più limpido

come esso brilla,

e raggiante d'una luce stellare

si leva verso l'alto?

Non lo vedete?

Come il cuore a lui

baldanzosamente si gonfia,

e pieno e maestoso

nel petto gli sgorga?

Come alle labbra,

voluttuosamente miti,

\section{segue nota 24}

per intero. Ora però il canto di morte s'immette direttamente nel suo svolgimento 'rotatorio' (ossia in progressioni) e a climax che avevamo ascoltato nel tempo veloce conclusivo della scena d'amore (nota 13), sia pure debitamente rallentato. Torna anche la tonalità che aveva governato questa pagina del secondo atto: quella di $\mathrm{Si}$. Gli snodi strutturali dell'estasi sono dunque gli stessi di allora (sezione A, canto di morte + figura di Trasfigurazione: «Seht ihr's nicht? | Wie das Herz ihm»; sezione A': «Höre ich nur»; sezione B, basata sul motivo della Beatitudine, l'es. 14: «Heller schallend»). A cambiare radicalmente è però l'esito finale. Mentre nell'atto secondo la cadenza alla tonica veniva scardinata con violenza, ora il motivo della Beatitudine sfocia per due volte in una cadenza plagale con flessione minore della sottodominante maggiore (Mi-Solq-Si), che ci conduce morendo alla tonica: un topos di Wagner - secondo Hermann Danuser, il suo personale "simbolo musicale della fine», che non a caso Wagner collocherà anche nelle battute estreme del Crepuscolo degli dèi e perfino nella sua revisione dell'Olandese volante. La seconda volta, la cadenza incorpora l'es. 1: il profilo cromatico del Desiderio (Sol\#3-La La\# $\#_{3}-\mathrm{Si}_{3}$ : es. $\left.1 y\right)$ nel timbro dell'oboe, combinato con il Tristanakkord $\left(\mathrm{Fa}_{2}-\mathrm{Si}_{2}-\mathrm{Re}_{3}-\mathrm{Sol}_{3}:\right.$ es. $\left.1 z\right)$, conducendoli entrambi alla risoluzione sulla tonica di Si (vedi le parti di archi, oboe e corno inglese nell'es. 28). La tonica conclusiva è sussurrata (pianissimo) nel tutti dell'orchestra, dal quale Wagner elimina significativamente il solo corno inglese, e la sua voce così pungente e caricata di dolore lungo le pagine della partitura. E finalmente, con questa cadenza plagale il Tristan und Isolde raggiunge una meta che non è simultaneamente un nuovo desiderio: al contrario, per dirla con Wagner, è l'«appagamento più beato dell'ardente brama [die seligste Erfüllung des glühenden Sehnens]». E forse, anche di quella degli spettatori del Tristan.

ESEMPIO 28 (III, bb. 1695-97)

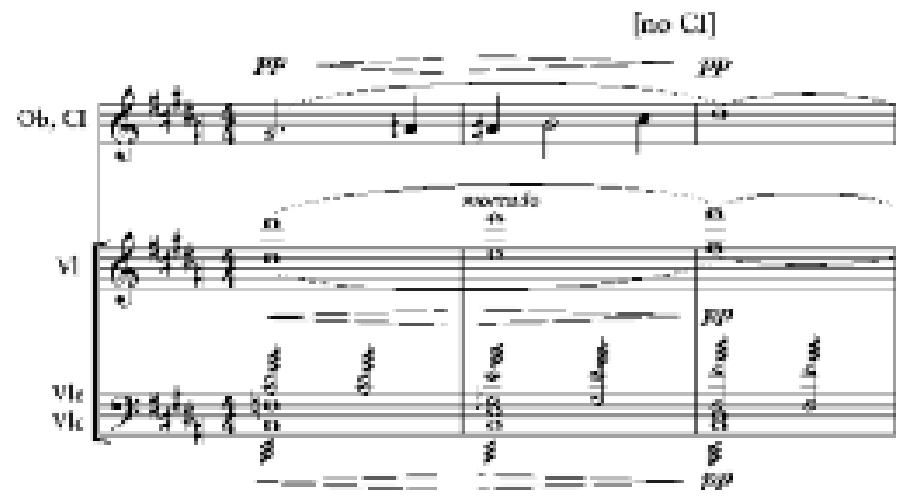

LXIV GS: «wie er leuchtet, | wie er minnig | immer mächt'ger, | Stern-umstrahlet | hoch sich hebt: | seht ihr, Freunde, I säh't ihr's nicht?». 
süßer Atem

sanft entweht: -

Freunde, seht -

fühlt und seht ihr's nicht? -

Höre ich nur

diese Weise,

die so wunder-

voll und leise,

Wonne klagend

alles sagend,

mild versöhnend

aus ihm tönend,

in mich dringet,

auf sich schwinget,

hold erhallend

um mich klinget? ${ }^{\mathrm{LXV}}$

Heller schallend,

mich umwallend,

sind es Wellen

sanfter Lüfte?

Sind es Wolken ${ }^{\text {LXVI }}$

wonniger Düfte?

Wie sie schwellen,

mich umrauschen,

soll ich atmen,

soll ich lauschen?

Soll ich schlürfen,

untertauchen,

süß in Düften

mich verhauchen?

In dem wogenden Schwall,

in dem tönenden Schall, ${ }^{\text {LXVII }}$

in des Welt-Athems

wehendem All -

ertrinken -

versinken -

unbewußt -

höchste Lust!

(Wie verklärt sinkt sie sanft in Brangänes Armen auf Tristans Leiche. - Große Rührung und Entrücktheit unter den Umstehenden. Marke segnet die Leichen. - Der Vorhang fällt langsam.) un dolce respiro

lievemente sfugge:...

Amici! Vedete!

Non lo sentite, non lo vedete?

Odo io soltanto

questa melodia,

che così mara-

vigliosa e sommessa,

voluttà lamentosa

tutto esprimente

dolce conciliante,

da lui risuonando

penetra in me,

e verso l'alto si libra

e dolce echeggiando

intorno a me risuona?

Queste armonie più chiare

che mi circondano,

sono forse onde

di miti aure?

Sono forse nubi

di voluttuosi vapori?

Come esse si gonfiano

e mi circondano del loro sussurro,

debbo io respirarle,

prestar loro ascolto?

A sorsi beverle,

sommergermici?

Dolcemente in vapori

dissiparmi?

Nell'ondeggiante oceano

nell'armonia sonora,

del respiro del mondo

nell'alitante Tutto...

naufragare,

affondare...

inconsapevolmente...

suprema letizia!

(Isolda, come trasfigurata, cade dolcemente, tra le braccia di Brangania, sul cadavere di Tristano. Grande commozione e rapimento tra gli astanti. Marke benedice i cadaveri. - La tela cala lentamente.)

\footnotetext{
LXV GS: «auf sich schwingt, | in mich dringt, I hold erhallend I um mich klingt?».

LXVI GS: «Wogen».

LXVII GS: «In des Wonnemeeres | wogendem Schwall, | in der Duft-Wellen I tönendem Schall,».
} 


\section{L'orchestra}

\begin{tabular}{|c|c|}
\hline 3 flauti (III anche ottavino) & 4 corni \\
\hline 2 oboi & 3 trombe \\
\hline corno inglese & 3 tromboni tenor-bassi \\
\hline $\begin{array}{l}2 \text { clarinetti } \\
\text { clarinetto basso }\end{array}$ & tuba bassa \\
\hline 3 fagotti & $\begin{array}{l}1 \text { coppia di timpani } \\
\text { triangolo }\end{array}$ \\
\hline arpa & piatti \\
\hline violini I & Musica di scena: \\
\hline violini II & 3 trombe \\
\hline viole & 3 tromboni \\
\hline violoncelli & 6 corni (o più se possibile) \\
\hline contrabbassi & corno inglese (o Alpenhorn) \\
\hline
\end{tabular}

Com'è noto, Tristan und Isolde deve la sua stesura ai lunghi anni 'sabbatici' presi da Wagner dal suo Siegfried. È dunque interessante misurare le forze orchestrali del Tristan con quelle che Wagner aveva messo in campo per la «seconda giornata» del Ring des Nibelungen. Balza subito agli occhi un alleggerimento dell'impressionante potenziale di volume sonoro di Siegfried, nella direzione di un organico più 'normale': una 'normalità', per inciso, che avrebbe dovuto ispirare il Tristan nel suo complesso, secondo le intenzioni originarie (e invero ottimistiche) di Wagner.

Scompaiono innanzitutto le poderose Tenortuben e Basstuben, espressamente concepite da Wagner (forse ispirato dal saxhorn di Adolphe Sax) per ispessire il suono del Ring nel registro medio. E, con le 'tube wagneriane', viene eliminato dall'organico anche il secondo quartetto di cornisti, ai quali esse vengono affidate. Dalle file degli ottoni si volatilizzano anche le altre novità dell'Anello: la tromba bassa e il trombone contrabbasso, così come quella tuba contrabbassa utilizzata come corposo sostegno ai corni e alle tube wagneriane.

Nel Ring, uno dei motivi di queste aggiunte era il desiderio di disporre di 'famiglie' timbriche omogenee di quattro strumenti: famiglie che ora vengono ridimensionate a gruppi di tre anche nei legni, con l'eliminazione dell'ottavino (ora suonato solo dal III 
flauto), di un oboe e di un clarinetto. Quanto ai fagotti, l'antipatia di Wagner verso i vecchi modelli di controfagotto gli aveva sconsigliato di allargare questo gruppo già nell'Anello (la diffidenza fu superata solo nell'ottobre 1879, quando Wagner udì il suono del Kontrafagott di Heckel: in tempo per inserirlo nell'organico di Parsifal).

Energicamente sfoltite anche le arpe, che ci avevano regalato il monumentale finale del Rheingold o i bagliori dell'Incantesimo del fuoco di Walküre. Lo stesso può dirsi delle percussioni: addio, ad esempio, al timbro del Glockenspiel, che ci regalerà qualcuno dei pochi tocchi di leggerezza della futura Götterdämmerung.

Questa contrazione è almeno parzialmente compensata dalla musica di scena (oltretutto rinforzabile a piacimento, nel caso dei sei corni che spandono la loro eco di Jagdhörner, di strumenti da caccia, nella prima scena dell'atto secondo). E da un timbro nuovo, che Wagner insiste gli sia concesso negli allestimenti del Tristan: timbro che dovrebbe sposarsi con la gioiosa melodia intonata dietro le quinte alla vista della nave di Isolde. Essa è assegnata in partitura al corno inglese, con la precisazione che lo strumento dovrebbe essere rinforzato da un certo numero di legni (oboi e clarinetti) a seconda dell'acustica della sala, in modo da produrre «l'effetto di uno strumento naturale molto vigoroso, come l'Alpenhorn [die Wirkung eines sehr kräftigen Naturinstrumentes, wie das Alpenhorn]». Ma di gran lunga preferibile sarebbe, secondo Wagner, la fabbricazione di un apposito Alpenhorn sulla falsariga di quelli svizzeri.

A tal proposito, sono divertenti i problemi 'ginnico-logistici' discussi da Wagner in un'apposita nota della partitura, legati al musicista che suona il corno inglese in orchestra. Lungo la prima scena dell'atto terzo, infatti, lo strumentista è dietro le quinte a suonare l'«antica canzone» della cornamusa; e tuttavia, poche pagine di partitura dopo le sue ultime note con la melodia dell'Alpenhorn, la sua presenza è richiesta tra gli orchestrali (il corno inglese è infatti coinvolto già dalle primissime battute della seconda scena). Il rapido spostamento potrebbe causare qualche intoppo. Pertanto, il suggerimento di Wagner è quello di affidare l'elementare melodia dell'Alpenhorn a un altro musicista, in modo da dare la possibilità al corno inglese di riprendere con tutta calma la sua posizione in orchestra. Una cautela comprensibile: se dietro le quinte è stato davvero usato l'Alpenhorn, immaginiamo l'imbarazzo del musicista che se lo ritrova distrattamente tra le mani, invece del corno inglese, una volta ridisceso a perdifiato nel golfo mistico... 


\section{Le voci}

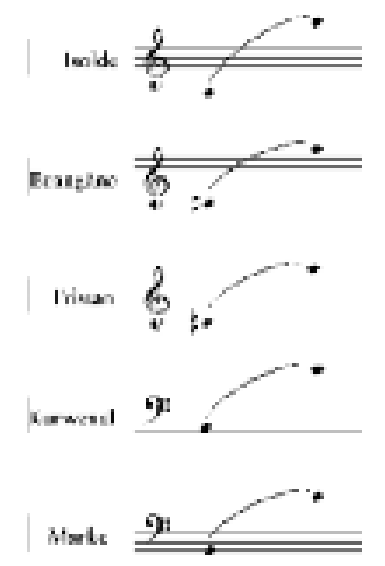

Tristan und Isolde reca già impresso nel titolo il delicato equilibrio che solo può garantirgli il successo. Due nomi per due ruoli equipollenti, che si spartiscono in modo abbastanza bilanciato la responsabilità della tenuta del dramma - anche se, evidentemente, non i minuti di canto. Duetti a parte, la presenza (scenica e vocale) di Isolde domina il lunghissimo atto primo e si impossessa della conclusione dell'intero lavoro. Tristan, dal canto suo, è praticamente solo al centro dei riflettori lungo tutta la prima, vasta scena del terzo - «il momento culminante dell'opera", come argomenta plausibilmente Joseph Kerman. Ma non è solo questione di tempo o di esposizione nei momenti cruciali: su entrambi pesa anche la complessità di un'evoluzione psicologica che deve necessariamente tradursi in timbro e in suono. L'Isolde selvaggia della prima scena o quella sostenuta e ironica del primo dialogo con Tristan (I.5) non sono ovviamente l'Isolde ardente dell'atto secondo, e meno ancora quella (vocalmente) 'trasfigurata' di «Mild und leise» che porta a compimento il dramma (e non di rado capita appunto di sentire voci a disagio con l'una o l'altra di queste sfaccettature del personaggio). Quanto a Tristan, la sola scena del 'delirio' implica la capacità di recitare credibilmente un percorso (freudiano ante litteram) di scoperta di sé, senza istrionismi o isterismi macchiettistici - e, si capisce, non è davvero poco. Traiettorie psichiche che diventano anche traiettorie musicali: l'Isolde di "Entartet Geschlecht» (I.1) è la stessa che, qualche ora più tardi, deve tenere e 'smaterializzare' in pianissimo il Fa\# $\#_{4}$ alla fine dell'opera. E forse, sotto il profilo musicale, ancora più esigente è la parte del tenore: cui si chiedono volume e pienezza di suono (inevitabile pensare a un Lauritz Melchior o a un Wolfgang Windgassen) e un ricco corredo di nuances dinamiche (quei mortali appiattimenti della voce sul forte da Heldentenor caricaturale...), ma soprattutto la finezza di un 'delirare cantando' (dove l'accento cade appunto sul canto, senza nulla concedere a quella specie di Sprechgesang espressionistico che costituisce una deriva costante di molti interpreti wagneriani). Come se non bastasse, l'equilibrio Tristan-Isolde non è autosufficiente: il fallimento dei colleghi di palcoscenico rischia infatti di compromettere gli sforzi della coppia - a cominciare dai due delicati ruoli di 'spalla'. Diffi- 
cile per un'Isolde entrare nella parte, lungo l'atto primo, se si ritrova come interlocutrice una mediocre Brangäne - voce alla quale peraltro Wagner riserva pagine intensissime e cruciali (si pensi al «Welcher Wahn?» dell'atto primo, al 'canto di veglia' nel secondo, a «Sie wacht! Sie lebt!» nel terzo...). Un danno analogo lo producono i Kurwenal dalla vocalità troppo grossière o viceversa troppo solenne, ieratica: un danno ai Tristan di turno, s'intende, soprattutto lungo la prima scena dell'atto terzo. Per non dire dei Marke: che non sono molto presenti in scena, ma che talvolta riescono - con le loro sole forze - ad affossare l'atto secondo, con il loro «Tatest du's wirklich?». I dodici minuti del lamento del re occupano infatti una posizione decisiva e invero arrischiata. È un po' come se il duetto fra Cio-Cio-San e Pinkerton di Madama Butterfly sfociasse in una lunga tirata di Sharpless: mal eseguiti, quei minuti possono raffreddare irrimediabilmente gli entusiasmi del pubblico dopo la scena d'amore, per quanto incandescente sia stata resa dai suoi interpreti. E invece "Tatest du's wirklich?" può coronare superbamente l'atto, se il basso riesce a intonarlo - parafrasando una bella frase di Didier van Moere - quasi come fosse un Lied di una Winterreise immaginaria. 


\title{
Tristan und Isolde in breve
}

\author{
a cura di Gianni Ruffin
}

Per la storia della musica, del teatro musicale e dell'arte tout court, il 10 giugno 1865 fu una data determinante: al «Königliches Hof- und Nationaltheater» di Monaco di Baviera fu rappresentata Tristano e Isotta di Richard Wagner. Che cosa avesse in mente l'artefice nel concepire un simile lavoro lo illustra nel modo più lampante una sua celeberrima lettera a Liszt nel 1854: «poiché in vita mia non ho mai gustato la vera felicità dell'amore, voglio erigere al più bello dei miei sogni un monumento nel quale dal principio alla fine sfogherò appieno questo amore. Ho sbozzato nella mia testa un Tristano e Isotta; un concetto musicale della massima semplicità, ma puro sangue; col bruno vessillo che sventola in fine del dramma, voglio avvolgermi per morire!». L'idea era chiarissima, la «tinta» anche: non restava (si fa per dire) che renderla concreta, mettere nero su bianco l'intuizione artistica e drammatica.

La scelta del modello letterario cui attingere era non a caso caduta sul più atipico dei capolavori medievali: l'incompiuto omonimo poema scritto nel 1205-1210 da Gottfried von Straßburg, un testo che «rispetto al suo tempo ed al suo mondo possiamo tranquillamente definire una mostruosità", scrive Peter Wapnewski, argomentando: «là dove [...] la stella del destino viene strappata giù dalla volta celeste e dalla sfera del divino e imperiosamente accasata nel singolo petto, là il Medioevo è finito". In un certo senso fortuna volle che il capolavoro di Gottfried rimanesse incompiuto: Wagner ebbe libera scelta nel delinearne la conclusione, compendiando nel finale la più sconvolgente concezione dell'amore, della vita e della morte mai fino ad allora apparsa.

Il modo in cui il Tristan wagneriano affronta (e porge) il tema dell'amore ha qualcosa di assoluto, come una trasgressione, per così dire, altamente morale: destino e amore vi coincidono assolutamente, in un modo tale che delitto sarebbe non assecondarli, o, peggio, ostacolarli. Solo una categoria freudiana come quella definita dal concetto di «regressione» sembra in grado di restituirci il senso dell'analogia profonda che lega l'idea di amore a quella della pulsione di morte: la morte comune dei due amanti è infatti presentata nel Tristan non alla stregua della luttuosa fatalità che, in molte opere romantiche, più o meno accidentalmente, interviene come sola possibile via d'uscita da una situazione senza rimedio, bensì come l'assolutamente necessaria conseguenza dell'amore, il suo compimento.

Assecondando un destino che li sovrasta, li travolge, e del quale si fanno entusiasticamente carico, i due amanti obliano ogni vincolo sociale, ogni senso d'appartenenza, qualsiasi legame d'amicizia. I valori fondativi dell'etica cavalleresca (onore, gloria, rispetto, fedeltà, altruismo) sono tutti presentati come simboli e frutti della vanità «diurna» e della falsità delle convenzioni sociali. Contrapposta alle meschine e superficiali menzogne del mondo, la sola verità che avvince i due amanti è l'anelito all'eterno indistinto della notte e della morte. Scriverà Nietzsche che "ogni gioia vuole eternità»; oggetto ultimo del desiderio di Tristan e di Isolde sarà dunque la morte perché solo la morte è eterno superamento della finitezza, dell'isolamento dell'io: solo 
nel grembo della notte eterna, infatti, la reciproca unione dei due amanti sarà assoluta e definitiva. Per capire a fondo questo capolavoro bisogna avere il coraggio di ammettere che il suo è un finale positivo.

Molteplici furono le istanze, le esperienze, le sollecitazioni confluite in simile concezione. Sicuramente un ruolo importante spettò all'esperienza biografica. Il riferimento, ben noto, è alla tempestosa vicenda sentimentale con Mathilde Wesendonck, della quale ricorderemo i Lieder musicati da Wagner, fra cui i due "studi per Tristan», Träume e Im Treibhaus, il primo dei quali Wagner definì «la cosa più bella che ho scritto (tremo sino alle radici più profonde dei miei nervi quando lo sento)».

Pertinente è anche il riferimento a Schopenhauer ed al suo pessimismo cosmico, purché si tenga presente l'ambivalenza, il risvolto terribilmente positivo poc'anzi spiegato della volontà di morte di Tristan e di Isolde. Non secondario dovrebbe risultare inoltre il richiamo alla metafisica della musica (intesa come diretto riferimento all'essenza del mondo) sviluppata dalla filosofia schopenhaueriana, che identifica in quest'arte la manifestazione dell'oscura pulsione della «volontà»: impossibile non evocare, di fronte a simile idea, la travolgente fluvialità dell'orchestra wagneriana che - secondo la definizione wagneriana del Tristan come «azioni della musica divenute visibili» - «trascina» i due amanti al compimento del destino.

Un influsso provenne anche dalla mitologia romantica d'amore, com'è ovvio: meno ovvio è notare come, essendo quella di amore, in Tristan, un'idea assoluta e totalizzante, essa non si limiti all'aspetto spirituale, ma coinvolga anche quello carnale. Senz'altro pertinente è anche il richiamo al retroterra filosofico fondante il romanticismo tedesco; Tristan ne implica concetti fondamentali come Zerrissenheit (il dissidio, il sentimento profondo di una lacerazione fra io e mondo) e Sehnsucht (lo struggimento, il «male del desiderio», il «desiderio infinito» che, in quanto infinito, si appaga del proprio inappagamento: concetto del quale il più diretto traslato musicale è raffigurato nell'ambiguità armonica - e nella correlativa inesausta tensione - del cosiddetto Tristanakkord).

Epocali furono le conseguenze del Tristano sull'arte, sulla musica, sul teatro, sulla filosofia, forse persino sulla vita di molti... è da sottolineare come, se assoluta è la portata del capolavoro in ordine a contenuti di tale entità, ciò si verifica perché di livello altrettanto assoluto è la risposta che alle sollecitazioni concettuali del testo ha saputo dare il compositore. Di particolare evidenza è l'originalità della concezione armonica: non meno che massiccio è il ricorso alla dissonanza, alla risoluzione eterodossa, al cromatismo. Con tali mezzi Wagner si spinge fino a delineare tensioni sonore estreme, paradossalmente ad un tempo dolcissime e dilaceranti, in più d'un caso di fatto inspiegabili nei termini dell'armonia tradizionale. Poderosa è la mobilitazione delle risorse sinfonico-orchestrali in una musica fluviale e travolgente che, dei trasgressivi contenuti notturni del Tristan und Isolde, appare come la sola, e conseguente, possibile espressione. 


\section{Argomento - Argument - Synopsis - Handlung}

\section{Argomento}

\section{ATTO PRIMO}

In mare, sul ponte della nave di Tristano, durante la traversata dall'Irlanda alla Cornovaglia. Isotta è sulla tolda della nave che la sta portando dall'Irlanda alla Cornovaglia. Tristano la scorta da re Marke, suo zio, cui Isotta deve andare in sposa. Un marinaio intona una canzone in cui si accenna ad una «vergine irlandese», e alle orecchie di Isotta le parole di quel canto suonano come un insopportabile oltraggio alla sua condizione di principessa. Quando l'ancella Brangania le annuncia che la nave sta per giungere in Cornovaglia, Isotta inveisce furiosa contro il proprio popolo, colpevole di essersi piegato vilmente al nemico.

Nella seconda scena Tristano è anch'egli sul ponte della nave e fissa assorto il mare, accanto al fido scudiero Kurwenal. Isolde ordina a Brangania di ingiungere a Tristano di renderle omaggio, visitandola nella sua tenda. Tristano rifiuta cortesemente, e Kurwenal spiega a suo modo a Brangania il motivo di tale diniego: Tristano è un eroe, ha ucciso l'irlandese Morold, promesso sposo di Isotta, poiché egli vessava la Cornovaglia, terra di re Marke, imponendo iniqui tributi; ora, dopo l'annientamento di Morold, Tristano porta Isotta a re Marke in sposa come segno della propria vittoria. Saputo del racconto beffardo di Kurwenal, Isotta inveisce contro Tristano ed il suo seguito e narra all'ancella di quando una piccola nave aveva portato sulle coste dell'Irlanda un uomo gravemente ferito, che ella aveva preso a curare con le arti magiche ereditate dalla madre. Nell'uomo Isotta aveva riconosciuto Tristano e subito aveva progettato di ucciderlo per vendicare Morold, ma era stata vinta dallo sguardo dell'eroe, che aveva suscitato nel suo cuore un misto di attrazione e pietà. Aveva così deciso di strapparlo alla morte con le sue cure. Tristano, guarito, era partito, ma dopo poco era tornato per portare Isotta, principessa d'Irlanda, in isposa a Marke, un semplice vassallo.

Terminato il racconto, Isotta, furibonda al ricordo di quest'ultima ingiuriosa sopraffazione, decide di vendicarsi di Tristano offrendogli, con la scusa di un brindisi pacificatorio, un filtro velenoso. Kurwenal avvisa le due donne di prepararsi allo sbarco poiché la nave è ormai prossima alla costa, ma Isotta invita lo scudiero a mandare da lei Tristano per un brindisi di riconciliazione. Giunto l'eroe, Isotta gli porge la coppa preparata da Brangania. Entrambi bevono, ma invece della morte li coglie un'inestinguibile corrente di reciproca attrazione: l'ancella infatti, incapace di assecondare la distruttiva volontà di Isotta, ha versato nel liquido della coppa non il filtro venefico, ma il filtro dell'amore. Tristano ed Isotta, ormai avvinti da una passione incoercibile, si scambiano parole di estatico rapimento mentre le fanfare di benvenuto e le urla di giubilo della folla annunciano l'arrivo in Cornovaglia. 


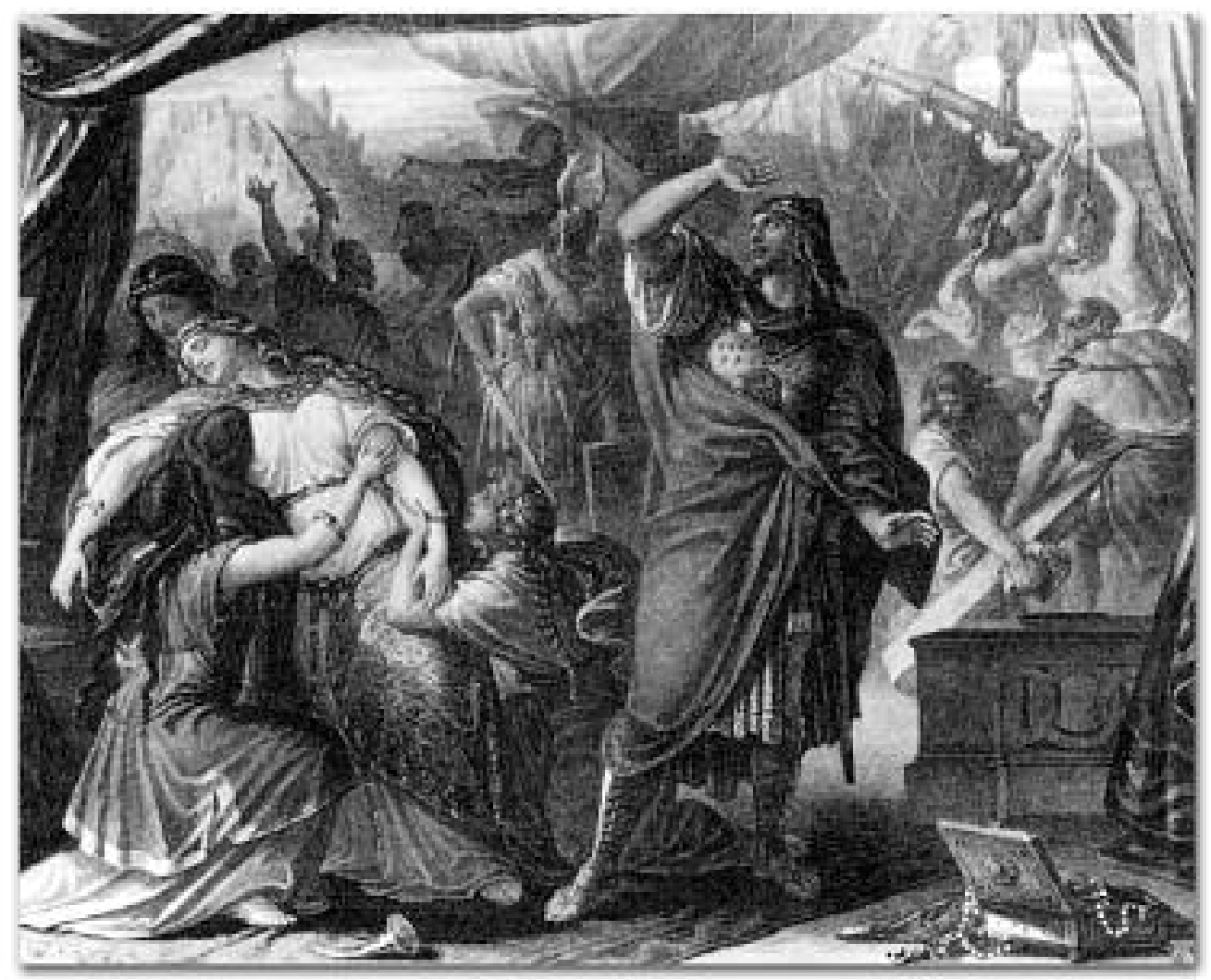

Tristan und Isolde (atto I). Litografia di Michael Echter (1812-1879) da un disegno di Angelo Quaglio II (18291890) per la prima rappresentazione assoluta, avvenuta a Monaco, Königliches Hof- und Nationaltheater, il 10 giugno 1865 .

\section{ATTO SECONDO}

Nel castello di re Marke in Cornovaglia. Nel giardino del castello di re Marke, in una ammaliante notte estiva, Isotta attende con impazienza l'arrivo dell'amato Tristano, mentre in lontananza echeggiano i suoni della battuta di caccia che re Marke sta compiendo con il suo seguito. Brangania invita però Isotta ad essere cauta e in particolare a diffidare di Melot, infido amico di Tristano e segretamente innamorato di Isotta, che ha organizzato la caccia come trappola per allontanare re Marke e sorprendere poi i due amanti clandestini.

Isotta liquida con impazienza quelli che ritiene sospetti infondati e spegne la torcia che arde nei pressi: sarà quello il segnale che annuncerà a Tristano che nulla ostacola il loro incontro. Quindi, in preda ad un crescente, irrefrenabile desiderio, attende impaziente l'amato, che finalmente giunge dalle tenebre della notte che protegge il loro incontro: essi si scambiano parole di estatico rapimento e, congiunti in un grandioso duetto, innalzano una sorta di inno alla notte, regno del nulla e dell'amore, opposto al mondo del giorno, infido e nemico. Mentre gli amanti sono al culmine della passione, Brangania, che vegliava guardinga sull'incontro notturno, annuncia l'irrompere sulla scena di re Marke, seguito da Melot e dal suo seguito. Il re, offeso dal tradimento di Trista- 
no, colto in flagrante con la sua promessa sposa, interroga addolorato l'amato nipote sui motivi del suo oltraggioso comportamento. Tristano non risponde, rapito nell'estasi amorosa e ormai dimentico del mondo e del suo stesso onore, e invita Isotta a seguirlo nel regno della notte. A quel punto Melot, colmo di gelosia, sfida Tristano a duello: i due estraggono le spade, ma l'eroe porge il proprio petto al nemico, che furiosamente lo trafigge.

\section{ATTO TERZO}

Nel castello di Tristano in Bretagna. Ai piedi di un grande albero giace Tristano ormai morente, vegliato dal fido Kurwenal, mentre risuona la desolata melodia della zampogna di un pastore. Lo scudiero chiede quindi al pastore di intonare un canto gioioso se, scrutando il mare, vedrà apparire la nave di Isotta. Alle parole di Kurwenal Tristano riprende lentamente coscienza ed in una sorta di delirio esalta il regno della notte, al quale ormai egli appartiene, anche se nel contempo il giorno lo richiama, per attendere Isotta, maledicendo il chiarore della torcia che divide i due amanti ed evita che possano ricongiungersi nel regno delle tenebre e del nulla. Quindi, arso dalla febbre, egli crede di veder giungere la nave di Isotta, ma alla sua angosciata richiesta a Kurwenal di confermargli l'arrivo del vascello dell'amata risponde la dolorosa melodia del pastore. Tristano ricorda allora che udì quella canzone da fanciullo quando i suoi genitori morirono e d'improvviso è colto dalla visione di Isotta, che lo raggiunge solcando il mare. Ma un nuovo gioioso motivo del pastore annuncia che la nave di Isotta sta realmente raggiungendo la costa. In preda ad una crescente eccitazione, Tristano si strappa le bende dalla ferita anelando al ricongiungimento con l'amata. Quando Isotta finalmente lo raggiunge, egli spira tra le sue braccia.

Irrompono quindi in scena Brangania e Melot, che viene ucciso da Kurwenal. Giunge anche re Marke che, saputo dell'inganno del filtro, era giunto per perdonare e riunire i due amanti e ora lamenta affranto la desolata scena di morte che lo accoglie. Isotta, estranea ormai a ciò che accade attorno a lei, anela solo a ricongiungersi a Tristano oltre la soglia del giorno ed in un lungo monologo conclusivo ella si trasfigura e muore accanto all'amato, mentre un luminoso e sereno accordo in maggiore conclude l'opera.

\section{Argument}

\section{PREMIER ACTE}

En pleine mer, sur le pont du navire de Tristan, pendant la traversée d'Irlande en Cornouailles. Iseult se trouve sur le pont du navire qui l'emmène d'Irlande en Cornouailles. Tristan l'escorte jusque chez son oncle, le roi Marc, qu'elle doit prendre pour époux. Un marin entonne une chanson qui fait allusion à une «vierge irlandaise». Les paroles de ce chant résonnent aux oreilles d'Iseult comme un outrage insupportable à sa condition de princesse. Lorsque sa servante Brangäne lui révèle que le navire s'apprête à aborder en Cornouailles, Iseult lance des invectives contre son peuple qui s'est lâchement plié aux volontés de l'ennemi.

Dans la deuxième scène, Tristan se trouve aussi sur le pont du navire et il fixe la mer, absorbé dans ses pensées; à ses côtés se tient son fidèle écuyer, Kurwenal. Iseult ordonne à Brangäne d'enjoindre Tristan à venir lui rendre hommage en daignant lui accorder une visite. Tristan refuse et Kurwenal explique à Brangäne les raison de son refus: Tristan est un héros; il a tué l'Irlandais Morold, le fiancé d'Iseult, parce que ce dernier infligeait aux habitants de la Cornouailles, terre de Tristan et du roi Marc, des impôts exorbitants. Après avoir mis fin aux jours de Morold, Tristan conduit Iseult au roi Marc, en signe de victoire. Lorsqu'Iseult entend le récit railleur de Kurwenal, 
elle peste contre Tristan et sa suite et elle raconte alors à sa servante qu'un petit bateau avait amené un jour sur les côtes d'Irlande un homme grièvement blessé, qu'elle soigna grâce aux pratiques magiques que sa mère lui avait transmises. Iseult, qui avait bien reconnu Tristan en cet homme, avait songé dans un premier temps à le tuer, pour venger Morold, mais elle finit par céder au regard du héros, qui éveilla en son coeur un mélange d'attirance et de pitié. Elle décida donc de l'arracher à la mort en lui prodiguant ses soins. Une fois guéri, Tristan partit mais il revint peu de temps après, pour la conduire, elle qui était princesse d'Irlande, au roi Marc, qu'elle devait épouser alors qu'il n'était qu'un simple vassal.

À la fin de son récit Iseult, furieuse devant cet abus offensant, décide de se venger de Tristan en lui offrant une boisson empoisonnée, sous prétexte de prendre un verre avec lui en signe de paix. Kurwenal prévient les deux femmes qu'il est temps de se préparer, car le navire approche des côtes, mais Iseult demande à l'écuyer de lui envoyer Tristan, pour boire à leur réconciliation. Tristan arrive et Iseult lui offre la coupe préparée par Brangäne. Tous deux boivent ce breuvage mais au lieu de trouver la mort, ils se sentent saisis d'une attirance réciproque: en effet, incapable de suivre la volonté destructrice d'Iseult, la servante avait versé dans la coupe non pas un poison, mais un philtre d'amour. Tristan et Iseult, désormais en proie à une passion effrénée, s'échangent des paroles brûlantes d'amour, tandis que les fanfares de bienvenue et les cris de joie de la foule annoncent l'arrivée en Cornouailles.

\section{DEUXIÈME ACTE}

Dans le château du roi Marc, en Cornouailles. Dans le parc du château du roi Marc, au cours d'une douce nuit d'été, Iseult attend avec impatience l'arrivée de son bien-aimé Tristan, tandis qu'on entend au loin les cris de la battue à laquelle se livrent le roi Marc et sa suite. Brangäne invite cependant Iseult à être prudente et à se méfier tout particulièrement de Melot: en effet, ce dernier n'est pas un ami loyal; il aime Iseult en secret et c'est lui qui a organisé cette chasse nocturne pour pouvoir éloigner le roi Marc et surprendre ainsi les deux amants clandestins.

Mais Iseult n'a cure de ces soupçons, qui sont à ses yeux dénués de fondement et elle s'empresse d'éteindre le flambeau qui luit à ses côtés. Tel est le signal qui laisse entendre à Tristan que plus rien ne s'oppose à leurs retrouvailles. L'amant, qui attendait impatiemment, animé d'un désir croissant et sans mesure, rejoint sa bien-aimée dans les ténèbres de la nuit, qui enveloppent leur rencontre d'un halo protecteur; ils échangent des paroles pleines d'extase et réunis en un duo grandiose, ils entonnent un hymne à la nuit, le royaume du néant et de l'amour, qui s'oppose au monde du jour, trompeur et ennemi. Tandis que les amants parviennent au comble de la passion, Brangäne, qui veillait prudemment sur leur rencontre, annonce d'un cri soudain l'irruption sur scène du roi Marc, suivi de Melot et de sa cour. Le roi, profondément blessé à la vue de la trahison de Tristan, qu'il surprend en flagrant délit avec sa future épouse, demande à son cher neveu, la mort dans l'âme, les raisons de son comportement outrageux. Tristan ne répond pas, car son extase amoureuse l'emporte et lui fait oublier le monde et son code de l'honneur, et il invite Iseult à le suivre dans le règne de la nuit. Mais Melot, dont la jalousie atteint le paroxysme, provoque Tristan en duel; tous deux tirent l'épée, mais le héros livre sa poitrine à l'ennemi qui le transperce.

\section{TROISIÈME ACTE}

Dans le château de Tristan, en Bretagne. Au pied d'un grand arbre gît Tristan; il est mourant et Kurwenal le veille, fidèlement, tandis qu'on entend un berger jouer une triste mélodie sur sa cornemuse. L'écuyer demande à ce dernier d'entonner un air joyeux dès qu'il verra apparaitre au loin le navire qui transporte Iseult. Aux paroles de Kurwenal, Tristan reprend peu à peu conscience et, 
plongé dans une sorte de délire, il exalte le royaume des ténèbres, auxquelles il appartient désormais, mais il demande en même temps un retour temporaire au jour, en attendant qu'Iseult revienne; il maudit la lueur de la torche qui sépare les deux amants et les empêche de se retrouver dans le règne des ténèbres et de la nuit. En proie à la fièvre, il croit apercevoir le navire d'Iseult, mais lorsqu'il demande à Kurwenal, le coeur étreint par l'angoisse, de lui confirmer l'arrivée du vaisseau de sa bien-aimée, il entend pour toute réponse la douloureuse mélodie du berger. Tristan se souvient alors avoir entendu cette chanson lorsqu'il apprit, enfant, la mort de ses parents et il est soudain envahi par une vision où Iseult se précipite vers lui, bravant la mer. Mais un nouvel air joyeux, joué par le berger, lui annonce que le navire d'Iseult est véritablement sur le point d'aborder au port. Dans son délire croissant, Tristan arrache les bandes qui protègent sa blessure et aspire de toutes ses forces à retrouver sa bien-aimée. Mais lorsqu'Iseult le retrouve enfin, il expire entre ses bras.

Brangäne entre alors en scène, suivie de Melot qui meurt sous le fer de Kurwenal. Puis arrive aussi le roi Marc qui, ayant appris le subterfuge du philtre d'amour, était venu exprimer son pardon aux amants, mais déplore à présent, le coeur brisé, la scène funèbre qui se déploie sous ses yeux. Iseult, désormais étrangère à tout ce qui l'entoure, n'a plus qu'un seul désir: retrouver Tristan par delà le seuil du jour, et tout au long du monologue final, elle se transfigure peu à peu puis elle meurt, aux côtés de son bien-aimé, tandis que l'opéra s'achève sur un accord en majeur, empreint de lumière et de sérénité.

\section{Synopsis}

\section{ACT ONE}

The scene is laid on board Tristan's ship, during the crossing from Ireland to Cornwall. Isolde is on the deck of the ship which is carrying her from Ireland to Cornwall. Tristan is escorting her to his uncle, King Marke, whom she is to marry. Isolde bristles with rage when she hears a sailor singing a song about an «Irish virgin», for she considers his taunting words an insult to her royal lineage. When her attendant, Brangäne, tells her that the ship is approaching Cornwall, Isolde vents her anger at her people for having yielded to the enemy in such a cowardly way.

In the second scene, Tristan is standing on the ship's deck, moodily contemplating the sea, with his loyal squire, Kurwenal, beside him. Isolde orders Brangäne to summon Tristan to her quarters to pay his respects. Tristan refuses, and Kurwenal explains why: Tristan has heroically slain Isolde's former betrothed, the tyrannical Irishman Morold who, after conquering Cornwall (homeland of Tristan and King Marke), had crippled the populace with high taxes. Now that Morold is dead, Tristan is taking Isolde to King Marke as a token of his victory. Angered by Kurwenal's insolent manner, Isolde rails against Tristan and his retinue. She tells Brangäne how a small ship, bearing a gravely wounded man, had landed on Irish shores and that, having inherited her mother's magic potions, she had taken care of him. Realising that the man was Tristan, Isolde's initial plan had been to kill him; but the look in his eyes had stirred up a mixture of attraction and pity in her heart, and she had instead nursed him back to health. His strength restored, Tristan had departed, only to return soon afterwards to convey the Irish princess to be the bride of King Marke, a mere vassal.

After ending her tale, Isolde flares up anew at the recollection of Tristan's unjust and arrogant behaviour, and resolves to take her revenge on him. She will invite him to drink a toast to their reconciliation - but the cup of friendship will in fact contain poison. The ship is nearing the coast, 
and Kurwenal tells the women to prepare to go ashore. Isolde asks the squire to send Tristan to her, so that they may drink to the end of their strife. But Brangäne, unable to bring herself to comply with Isolde's wishes, has substituted a love potion for the poison. As soon as they drink the potion, the couple are overwhelmingly drawn to each other. Dizzy with longing, Tristan and Isolde ecstatically exchange endearments while, in the background, a loud fanfare and the jubilant cries of the crowd proclaim their arrival in Cornwall.

\section{ACT TWO}

King Marke's castle in Cornwall. It is a summer's night, and the air is full of magic. Isolde is in the garden of King Marke's castle, awaiting Tristan's arrival with mounting excitement. King Marke and his courtiers are out hunting, and the sound of their bugles echoes in the distance. Brangäne warns Isolde to be cautious and, above all, to beware of the treacherous Melot, who is secretly in love with her. Melot has organised the hunting party to persuade King Marke to leave the castle, knowing full well that on his return he will catch the lovers together.

Isolde brusquely dismisses her suspicion as unfounded, and extinguishes the torch burning on the castle wall, thereby signalling to Tristan that they can meet in safety. Isolde can scarcely conceal her growing desire as she impatiently awaits her lover. At long last, Tristan emerges from the dark, protective cloak of night. Falling rapturously into each other's arms, they launch into a majestic duet, a song of praise to the night - the kingdom of nothingness and of love, contrasting with the hostile, faithless day. As they reach the height of ecstasy, Brangäne shouts from her watching place that King Marke is approaching. The courtiers, led by Melot and King Marke, burst in and discover the lovers locked in an embrace. Deeply wounded by his nephew's betrayal, King Marke demands an explanation for his outrageous behaviour. Tristan does not answer; swept away by passion, he has banished all thoughts of the world and of his very honour, and he begs Isolde to follow him into the kingdom of night. Wildly jealous, Melot challenges Tristan to a duel, and the two men draw their swords. Tristan bares his breast, into it his foe immediately thrusts his weapon.

\section{ACT THREE}

Tristan's castle in Brittany. Mortally wounded, Tristan lies at the foot of a huge tree, attended by the faithful Kurwenal. A shepherd plays a melancholy strain on his pipe. Kurwenal asks the shepherd to keep watch for Isolde's ship, and to play a cheerful melody as soon as it comes into sight. At the sound of Kurwenal's voice, Tristan slowly regains consciousness. Lapsing into a trancelike state, he sings out in praise of the realm of night, which by now has claimed him, but revives briefly at the prospect of Isolde's arrival. He curses the glimmering torch which keeps the lovers apart, barring them from finding eternal joy in the sphere of darkness and nothingness. Burning with fever, Tristan is convinced that Isolde's ship is drawing towards the coast. He questions Kurwenal excitedly, only to be answered by the plaintive sound of the shepherd's pipe. Tristan recalls hearing the same tune as a boy, when his parents died; suddenly, he is blinded by a vision of Isolde sweeping through the waves to join him. The shepherd's pipe gives out a lively melody, announcing that Isolde's ship is landing. Isolde rushes toward Tristan who, desperate to be reunited with his loved one, frenziedly tears the bandages from his wound. But when Isolde finally reaches him, he dies in her arms.

Brangäne appears, followed by Melot, whom Kurwenal kills. Also King Marke enters: having been informed of the exchange of potions, he intends to pardon the lovers. Instead, he is greeted with a chilling scene that fills him with despair. Isolde, no longer aware of what is happening, 
yearns only to join Tristan in the kingdom of night. In a prolonged final monologue, she is transfigured. As Isolde sinks lifeless over her lover's body, a pure, peaceful note rings out, highlighting the tragic conclusion of the opera.

\section{Handlung}

\section{ERSTER AKT}

Auf dem offenen Meer, an Deck des Schiffes Tristans, auf der Überfahrt von Irland nach Cornwall. Isolde ist auf dem Oberdeck des Schiffes, das sie von Irland nach Cornwall bringt. Tristan geleitet sie zu seinem Onkel, König Marke, dem sie als Braut versprochen ist. Vom Maste her erklingt das Lied eines jungen Seemanns, der seiner irischen Maid in Sehnsucht gedenkt. Die Worte des Liedes erscheinen Isolde, der Prinzessin, grob und wie eine Beleidigung. Als die Magd Brangäne Isolde mitteilt, dass der Strand Cornwalls bald erreicht sei, findet dieselbe nur böse Worte für ihr eigenes Volk, dem sie vorwirft sich auf niederträchtige Art dem Feinde unterworfen zu haben.

Im zweiten Bild sieht man Tristan, in Begleitung seines getreuen Knappen Kurwenal, auf dem Deck des Schiffes, in Gedanken versunken auf das Meer schauend. Mit dem Befehl ihr, Isolde, in ihrem Zelt aufzuwarten schickt sie Brangäne zu Tristan. Mit höflichen Worten weicht dieser aus und Kurwenal erklärt Brangäne den Grund der Ablehnung: Tristan sei ein Held da er den Irländer Morold, Verlobten Isoldes, getötet habe der Cornwall, Tristans und König Markes Heimat, mit ungerechten Abgaben geplagt habe. Als Zeichen seines Sieges bringe er nun Isolde König Marke als Braut. Isolde, unterrichtet von den höhnischen und zynischen Worten Kurwenals, ist verärgert über Tristan und seine Getreuen. Sie erzählt ihrer Magd von dem Tag, an dem ein kleines Schiff einen schwerverletzten Mann an die Küste Irlands brachte, der sich ihr, da sie die magischen Heilkünste ihrer Mutter geerbt habe, zur Heilung anvertrauen wollte. In diesem Mann habe sie Tristan erkannt und beschlossen ihn zu töten, um den Tod Morolds zu rächen. Aber als er die Augen geöffnet habe, sei sie von einem Gefühl des Mitleids erfüllt worden und habe daher beschlossen ihn zu pflegen und dem Tod zu entreißen. Gesundet sei Tristan nach Cornwall zurückgekehrt, bald jedoch wiedergekommen um sie, Isolde, irländische Prinzessin, als Braut Markes, ihrem einfachen Untertan, fortzuführen.

Verletzt durch dieses beleidigende Verhalten beschließt sie sich an Tristan zu rächen, in dem sie ihm, anstatt eines angekündigten Friedenstranks, einen Todestrank verabreichen will. Kurwenal kündet den beiden Frauen die nahe Landung an. Isolde bittet den Knappen, Tristan zu ihr zu schicken, um mit ihm vor der Landung noch Frieden zu schließen und einen Friedenstrunk zu teilen. Tristan erscheint, Isolde reicht ihm den von Brangäne bereiteten Kelch. Beide trinken, aber anstatt des erwarteten Todes, durchströmt sie ein unendliches Liebesgefühl, denn die Magd, unfähig der Weisung Isoldes zu folgen, hat statt des Todestrankes den Kelch heimlich mit dem Liebestrank gefüllt. Während die Musikkapelle und die Willkommensrufe der Menge die Ankunft des Schiffes ankündigen, tauschen Tristan und Isolde ekstatische Liebesworte aus.

\section{ZWEITER AKT}

Im Schloß von König Marke in Cornwall. Im Garten des Schlosses, in einer bezaubernden Sommernacht, harrt Isolde in sehnsuchtsbanger Erwartung des Geliebten, Tristan, während ferne Hornklänge König Marke und sein Gefolge zur Jagd rufen. Brangäne warnt Isolde vor Melot, dem treulosen Freund Tristans und heimlich verliebt in Isolde, der die nächtliche Jagd organisiert hat, um König Marke zu entfernen und dann die beiden heimlichen Geliebten zu überraschen. 
Isolde weist ungeduldig den Verdacht der Magd zurück und löscht die Fackel die in ihrer Nähe brennt, deren Verlöschen für Tristan das Zeichen ist, dass er nahen darf. Ungeduldig und erfasst von unbezwingbarem Sehnen erwartet sie den Geliebten, der endlich aus der tiefen Finsternis, die ihre Zusammenkunft schützen wird, erscheint. In ekstatischer Entrücktheit und vereint in einem überwältigendem Duett erheben sie einen Hymnus an die Nacht, als das Reich des Nichts und der Liebe, indes der Tag feindlich und treulos erscheint. Während sich die Geliebten traumverloren sich ihrer Passion hingeben, erschallt der Mahnruf Brangänes, die aufmerksam über die Zusammenkunft gewacht hat, der die Rückkehr König Markes mit seinem Gefolge und Melot ankündigt. Der König, verletzt vom Verrat Tristans, befragt den geliebten Neffen nach dem Warum seines Handelns. Tristan muss die Antwort schuldig bleiben. Überwältigt von der Liebe, die Welt und seine eigene Würde vergessend, bittet er Isolde ihm in das Land der Nacht zu folgen. Melot aber, von Eifersucht verzehrt, fordert ihn zum Zweikampf. Beide ziehen die Schwerter, doch Tristan stürzt sich in die Waffe des Gegners.

\section{DRITTER AKT}

Im Schloss Tristans in der Bretagne. Betreut von seinem ehrenhaften Kurwenal liegt Tristan sterbend im Schatten eines großen Baumes, während aus der Ferne eine elegische Hirtenschalmei erklingt. Kurwenal bittet den Hirten eine fröhliche Weise anzustimmen sobald sich das Schiff Isoldes auf dem Meer zeigt. Bei den Worten Kurwenals erwacht Tristan und beginnt, in einer Art Delirium, das Reich der Nacht, dem er schon angehört, zu preisen. Er bittet jedoch ihn vorerst noch dem Reich des Tages zurückzugeben, um auf Isolde warten zu können. Er verdammt das Licht der Fackel, das die Liebenden trennt und ihnen die Wiedervereinigung im Reich der Finsternis und des Nichts verweigert. Im Fieberwahn glaubt er das Schiff Isoldes nahen zu sehen. Seine angstvolle Frage an Kurwenal findet Antwort in der traurigen Hirtenweise. Tristan erinnert dieses Lied schon als Kind, als seine Eltern starben, gehört zu haben. Er sieht Isolde vor sich, die auf den Wellen schreitend zu ihm kommt. Plötzlich aber kündet die fröhliche Hirtenweise, dass das Schiff Isoldes wirklich naht. In rasender Ekstase rafft er sich auf, reißt den Verband von seiner Wunde und taumelt der Ersehnten entgegen, die ihn, sterbend, in ihren Armen auffängt.

Brangäne erscheint gefolgt von Melot, der von Kurwenal getötet wird. König Marke, unterrichtet vom Geheimnis des Liebestranks, gekommen um die Liebenden zu vereinen, steht erschüttert vor diesem Bild des Todes. Isolde, wie aus tiefer Ohnmacht erwacht, der Welt schon entrückt, hat nur den Wunsch sich mit Tristan zu vereinen. Nach einem langen Monolog stirbt sie neben dem Geliebten, während ein strahlender und reiner Dur-Dreiklang die Oper abschließt. 


\title{
Bibliografia
}

\author{
a cura di Riccardo Pecci
}

Visti i rapporti piuttosto stretti che legano il «gran Maestro del Vendramin» (e Tristan und Isolde) ai luoghi del nostro paese, colpisce il disinteresse ostentato - oggi più che mai - dall'editoria italiana per il fenomeno Wagner. Mentre nuovi libri compaiono col contagocce, i vecchi sono scomparsi inesorabilmente, uno dopo l'altro, dagli scaffali delle librerie (specializzate e non). E l'impressione è che non sia un arrivederci. Possiamo solo sperare si tratti della classica quiete prima della tempesta: quella editoriale, s'intende, che dovrebbe (auspicabilmente) scatenarsi l'anno venturo, in coincidenza con il duecentesimo compleanno di Richard Wagner.

Sia come sia, la falla rende attualmente il corpo a corpo con le lingue straniere quasi inevitabile. Un quadro complessivo relativamente aggiornato degli studi su Wagner è offerto dall'edizione 2001 del New Grove, ${ }^{1}$ dalla voce firmata da Barry Millington con apporti di John Deathridge, Carl Dahlhaus e Robert Bailey. Il contesto biografico, storico e culturale nel quale prese forma Tristan è ricostruito nelle molte monografie basate sullo schema 'vita e opere di Wagner', delle quali (almeno questo, per fortuna!) si può leggere una buona scelta in lingua italiana, anche se esclusivamente nelle biblioteche (poco o nulla è ormai in libreria): Mayer, Gutman, Westernhagen, Gregor-Dellin ${ }^{2}$ - da integrare, ora, con il recente volume di Köhler. ${ }^{3}$ Chi ami le spudorate agiografie, potrà deliziarsi anche con il 'vangelo' di Curt von Westernhagen. ${ }^{4}$ Gli spettatori veneziani potranno invece trovare particolarmente interessante la rievocazione di Wagner appunto come "gran Maestro del Vendramin», che si spegne sul Canal Grande di Venezia nel pomeriggio del 13 febbraio 1883, in procinto di festeggiare i suoi settant'anni. ${ }^{5}$ Per i cultori del dettaglio biografico,

\footnotetext{
1 The New Grove Dictionary of Music and Musicians, 29 voll., a cura di Stanley Sadie, London, MacMillan, $2001^{2}$.

2 Hans MaYer, Richard Wagner, Milano, Mondadori, 1967 (trad. di Anmerkungen zu Wagner, Frankfurt am Main, 1966); ROBERT W. GUTMAN, Wagner, l'uomo, il pensiero, la musica, Milano, Rusconi, 1983 (trad. di Richard Wagner. The Man, his Mind, and his Music, New York, Harcourt Brace Jovanovich, 1968); CuRT VON Westernhagen, Wagner. L'uomo, il creatore, Milano, Mondadori, 1983 (trad. di Wagner, ZürichFreiburg, Atlantis, 1979); MarTin Gregor-Dellin, Wagner, Milano, Rizzoli, 1983 (trad. di Richard Wagner. Sein Leben, sein Werk, sein Jabrhundert, München-Zürich, Piper, 1980).

3 JoAchIm KöHLER, Der Letzte der Titanen: Richard Wagners Leben und Werk, Berlin, Claassen, 2001 (trad. inglese: Richard Wagner: the Last of the Titans, New Haven and London, Yale University Press, 2004).

${ }^{4}$ Ossia Curt von Westernhagen, Wagner, Milano, Accademia, $1977^{2}$ (trad. di Wagner, ZürichFreiburg, Atlantis, 1968).

${ }^{5}$ Henry PerL, Richard Wagner in Venedig. Mosaikbilder aus seinen letzen Lebenstagen. Mit einem Vorworte und unter Benutzung der Beobachtungen des Herrn Dr. Friedrich Keppler, Augsburg, Gebrüder Reichel, 1883: trad. in Richard Wagner a Venezia, edizione italiana e tedesca a cura di Quirino Principe, Venezia, Marsilio, 2000, pp. 78 e 226.
} 
l'opera di riferimento è invece ancora quella di Ernest Newman. ${ }^{6}$ Né ci dobbiamo accontentare di questa tipologia libraria: una grande messe di informazioni è offerta anche da iniziative editoriali di altro genere. In particolare, si segnalano i vari Companions, firmati da squadre di specialisti, in grado di rispondere a molte delle curiosità che s'annidano invariabilmente nella testa dello spettatore: ultimo arrivato, il Cambridge Companion to Wagner, a cura autorevole di Thomas S. Grey. ${ }^{7}$ Utile al lettore italiano per un inquadramento del Tristan nella storia ottocentesca del teatro d'opera tedesco è anche una fatica recente di Principe. ${ }^{8}$ A completare l'offerta, si può contare anche su una discreta raccolta di materiale iconografico e documentario, relativo a Wagner e ai suoi contemporanei. ${ }^{9}$

Né si può ignorare la mole di scritti vergati dalla penna di Wagner - migliaia di pagine di saggi, diari, lettere tra le quali si celano documenti di grande significato per la comprensione di Tristan e della sua genesi. A coloro che possiedono un computer e leggono il tedesco segnaliamo un utilissimo strumento, che finalmente raduna in pochi grammi di plastica una parte cospicua di questo materiale, di provenienza tanto eterogenea: il ricchissimo CD-ROM curato da Sven Friedrich. ${ }^{10}$ Il CD-ROM accoglie tra l'altro buona parte dei 16 voll. delle Sämtliche Schriften und Dichtungen di Wagner (Leipzig, Breitkopf und Härtel, 1911-1916: ricordiamo comunque ai tradizionalisti che possono accedere a diverse edizioni - e ristampe - cartacee del corpus degli scritti e poemi di Wagner). ${ }^{11}$ In lingua italiana, meritano una lettura mirata l'autobiografia (di cui si contano due traduzioni), ${ }^{12}$ gli scritti teorici zurighesi ed altri scritti posteriori, ${ }^{13}$ da percorrere comunque con qualche buon sussidio interpretativo al proprio fianco. ${ }^{14}$

${ }^{6}$ ERnest NeWman, The Life of Richard Wagner, 4 voll., Cambridge, Cambridge University Press, $1976{ }^{2}$.

7 Cambridge, Cambridge University Press, 2008; lo precedono Peter BuRbidge e Richard SutTon, The Wagner Companion, London, Faber \& Faber, 1979; BarrY Millington, Wagner, London, Dent and Sons, 1984; John Deathridge e Carl Dahlhaus, The New Grove Wagner, New York, Norton, 1984; Ulrich Müller e PeTER WAPNEWSKI, Richard-Wagner-Handbuch, Stuttgart, Kröner, 1986 (trad. inglese Wagner Handbook, Harvard, Harvard University Press, 1992); The New Grove Wagner, a cura di Barry Millington, London, St Martins Press, 2001; The Wagner Compendium, a cura di Barry Millington, London, Thames \& Hudson, 20012.

${ }^{8}$ Quirino PrinciPe, Il teatro d'opera tedesco 1830/1918, Palermo, L'Epos, 2004.

${ }^{9}$ Herbert Barth, Dietrich Mack, EgON Voss, Richard Wagner. Leben und Werk in zeitgenössischen Bildern und Dokumenten, a cura di Egon Voss, Zürich, Atlantis, $1998^{3}$ (ed. inglese: Richard Wagner. A Documentary Study, New York, Thames and Hudson, 1984).

10 Richard Wagner, Werke, Schriften und Briefe, a cura di Sven Friedrich, Berlin, Direct Media, 2004.

${ }^{11}$ Ricordiamo almeno la rist. anastatica (Hildsheim, Olms, 1976) di ID., Gesammelte Schriften und Dichtungen, 10 voll., Leipzig, Fritzsch, 1887-88², e la successiva Jubiläumausgabe (uscita appunto nel centenario della morte di Wagner): ID., Dichtungen und Schriften, a cura di Dieter Borchmeyer, 10 voll., Frankfurt am Main, Insel, 1983. Per quanto riguarda il Tristan, una cernita di materiale facilmente reperibile online è EDWIN LINDNER, Richard Wagner über "Tristan und Isolde»: Aussprüche des Meisters über sein Werk aus seinen Briefen und Schriften zusammengestellt und mit erläuternden Anmerkungen, Leipzig, Breitkopf \& Härtel, 1912.

12 ID., Autobiografia, Milano, Dall'Oglio, 1983 e La mia vita, a cura di Massimo Mila, Torino, UTET, 1953 (poi anche Torino, EDT, 1982).

${ }^{13}$ L'opera d'arte dell'avvenire, trad. di Alfio Cozzi, introduzione di Paolo Isotta, Milano, Rizzoli, 1983; Una comunicazione ai miei amici, trad. di Francesco Gallia, Pordenone, Studio Tesi, 1985; quanto ad Oper und Dra$m a$, è sì accessibile ma in una versione da maneggiare con cautela - ossia quella ottocentesca preparata per la Biblioteca Artistica dei torinesi Fratelli Bocca: Opera e dramma, trad. italiana eseguita sulla seconda edizione tedesca da Luigi Torchi, Bocca, Torino, 1894, 19292. Fondamentali sono alcuni dei testi raccolti in RICHARD WAGNER, «Musikdrama». Scritti teorici sulla musica, trad. di Francesco Gallia, Pordenone, Studio Tesi, 1988 e ID., Scritti scelti, a cura di Dietrich Mack, introduzione di Ernst Bloch, Parma, Guanda, 1988.

${ }_{14}$ Ad esempio: DIETER BORCHMEYER, Richard Wagner: Theory and Theatre, Oxford, Clarendon Press, 1991 (trad. riveduta di Das Theater Richard Wagners. Idee-Dichtung-Wirkung, Stuttgart, 1982); THOMAS S. GreY, 
Ed ora, preoccupiamoci di accostarci più direttamente al teatro della maturità wagneriana. Il lettore italiano può tranquillamente cominciare dalle versioni commentate dei libretti wagneriani di Guido Manacorda, che offrono più di una semplice traduzione (purché, naturalmente, non gli faccia difetto il senso della storia). ${ }^{15}$ Ottimi sguardi d'insieme sono offerti dalla già citata monografia di Millington del 1984, che ora ci propone un (troppo) agile volumetto basato sulle sue voci wagneriane per il New Grove Dictionary of Opera (1992), arricchito tra l'altro dalla classica voce Leitmotif di Arnold Whittall. ${ }^{16}$ In italiano, è giocoforza iniziare dalle riflessioni di Dahlhaus. ${ }^{17}$ Dopo di che, si schiude l'oceano della cosiddetta Leitfadenliteratur: la tradizione, cioè, delle guide tematiche alle opere di Wagner, che cercano di dipanarne l'imbrigliata 'matassa' leitmotivica - raccontandoci, passo dopo passo, la trama dell'opera. In principio furono quelle celeberrime del barone Hans von Wolzogen (1848-1938), ripubblicate ininterrottamente per decenni. ${ }^{18}$ Nonostante l'età, le guide Wolzogen valgono una lettura: sono una pagina di storia, e hanno pur sempre gettato le fondamenta sulle quali è stata edificata gran parte della Leitfadenliteratur posteriore che non sempre, va detto, ha superato Wolzogen per cura del dettaglio. Della prima, storica ondata di guide wagneriane consigliamo quella di Max Chop, che ha ugualmente beneficiato di una traduzione italiana, e quella di Otto Neitzel, un discepolo di Liszt. ${ }^{19}$ All'interno di questo filone s'inscrive anche il popolarissimo volume del grande biografo di Wagner, Newman - un classico intramontabile, la cui traduzione è però fuori commercio ormai da troppi anni. ${ }^{20}$ The Wagner Nights è un volume impreziosito da un'accurata ricostruzione della genesi delle opere, che - nonostante qualche ruga - è tuttora candidato a ideale viatico dell'aspirante wagneriano.

Ma non possiamo neppure dimenticare gli addentellati filosofici del teatro di Wagner, e segnatamente del Tristan. Su Wagner interprete del Wille di Schopenhauer il lettore italiano può consultare un piccolo libro di Giovanni Piana ${ }^{21}$ e uno studio sulla controversia Wagner-(Schopenhauer)-Nietzsche, ${ }^{22}$ che nel titolo ammicca ai "documenti processuali» del Nietzsche contra Wagner - lettura consigliabilissima, assieme alle altre prose wagneriane del filologo-filosofo della Nascita della tragedia dallo spirito della musica. ${ }^{23}$ Una lettura curiosa (ma altamente istruttiva per com-

Wagner's musical prose: Texts and contexts, Cambridge, Cambridge University Press, 1995; MauRIZIO Giani, Un tessuto di motivi. Le origini del pensiero estetico di Richard Wagner, Torino, De Sono-Paravia, 1999.

15 Vedi la nostra introduzione all'edizione del libretto. 2006

16 Barry Millington, The New Grove Guide to Wagner and His Operas, Oxford, Oxford University Press,

17 Carl Dahlhaus, I drammi musicali di Richard Wagner, Venezia, Marsilio, 1984 (trad. di Die Musikdramen Richard Wagners, Hannover, Friedrich, 1971).

${ }_{18}$ Nel nostro caso, Hans von Wolzogen, Thematischer Leitfaden durch die Musik zu Richard Wagner's "Tristan und Isolde», nebst einem Vorworte über den Sagenstoff des Wagner'schen Dramas, Leipzig, Feodor Reinboth, $1888^{3}$.

19 Max СHOP [M. CHARLES], Vademecum für Wagnerfreunde. Führer durch Richard Wagner's Tondramen, Leipzig, Rossberg, 1893; OтTо NeITZEL, Richard Wagners Opern, Stuttgart-Berlin, Cotta'sche Buchhandlung, $1908^{4}$.

20 ERnest Newman, Le opere di Wagner, Milano, Mondadori, 1981 (trad. di The Wagner Nights, London, Putnam, 1949; pubblicato anche come The Wagner Operas, New York, Knopf, 1949, e ora Princeton, Princeton University Press, 1991).

${ }^{21}$ Giovanni Piana, Teoria del sogno e dramma musicale. La metafisica della musica di Schopenhaner, Milano, Guerini, 1997.

22 Guido Morpurgo Tagliabue, Nietzsche contro Wagner, Pordenone, Studio Tesi, 1993.

23 Friedrich Nietzsche, Scritti su Wagner: «Richard Wagner a Bayreuth» - «Il caso Wagner» - «Nietzsche contra Wagner», Milano, Adelphi, 1979. Sull'inesauribile triangolo Nietzsche-Cosima-Wagner vedi ora DIETER BORCHMEYER, Nietzsche, Cosima, Wagner. Porträt einer Freundschaft, Frankfurt, Insel, 2008. Per una visione più 
prendere rettamente la compassione 'schopenhaueriana' di Wagner) è la recente traduzione in lingua italiana della Lettera aperta contro la vivisezione. ${ }^{24}$

Un autentico compendio di approcci ermeneutici è poi il Wagner androgyne di Jean-Jacques Nattiez, che nondimeno privilegia il Ring. ${ }^{25} \mathrm{Al}$ rapporto di Wagner con la figura di Tristan e - più in generale - a quello di proiezione che lega il compositore ad alcuni dei suoi più grandi eroi è dedicato un paio di studi del germanista Peter Wapnewski, che sa trarre partito dalla sua dimestichezza con le fonti medievali. ${ }^{26}$ In tema di eroismo, si può consultare anche Simon Williams. ${ }^{27}$ Utili anche alcuni saggi (più o meno recenti) firmati dall'autorevole Dieter Borchmeyer. ${ }^{28}$

Non mancano ovviamente proposte sul piano più strettamente musicale: il significato della tecnica leitmotivica e le modalità narrative dell'orchestra di Wagner hanno ricevuto le attenzioni di almeno un paio di studiosi italiani. ${ }^{29}$ Cruciale è anche lo studio della tecnica wagneriana della strumentazione, oggetto di uno studio classico di Egon Voss. ${ }^{30}$ E determinante è il rapporto di Wagner con l'idolatrato Beethoven, sviscerato da una monografia di Klaus Kropfinger. ${ }^{31}$

Ed ora, accostiamoci specificamente al Tristan und Isolde. Il lettore italiano può tranquillamente cominciare dalla versione commentata di Guido Manacorda. ${ }^{32}$ È utile comparare questa traduzione con quella di Olimpio Cescatti o con quella di Franco Serpa, che ha anche volto in italiano l'abbozzo in prosa terminato da Wagner nell'agosto 1857.33 Chi volesse sapere di più della leggenda di Tristano potrebbe cominciare dalle voci di un buona guida al vasto corpo dei racconti medievali ${ }^{34}$ e poi immergersi direttamente nei frammenti della fine del XII secolo di Béroul e di Thomas d'Angleterre. ${ }^{35}$ Proseguendo ovviamente con il poema di Gottfried von Strassburg (del

provocatoria, ma estremamente documentata del rapporto fra il filosofo e la coppia di Bayreuth si veda JOACHIM KöHLER, Friedrich Nietzsche e Cosima Wagner, Milano, Pratiche, 1997 (trad. di Friedrich Nietzsche und Cosima Wagner. Die Schule der Unterwerfung, Berlin, Rowohlt, 1996).

${ }_{24}$ RichaRd WAGNER, Sulla vivisezione. Lettera aperta al signor Ernst von Weber, autore dello scritto «Le camere di tortura della scienza», a cura di Sandro Barbera e Giuliano Campioni, Pisa, ETS, 2006.

25 Jean-JaCques NaTTIEZ, Wagner androgino: saggio sull'interpretazione, Torino, Einaudi, 1997 (trad. di Wagner androgyne: essai sur l'interprétation, Paris, Christian Bourgeois, 1990).

26 PeTER WAPNEwSKI, Tristano, l'eroe di Wagner, Bologna, Il Mulino, 1994; ID., Der traurige Gott: Richard Wagner in seinen Helden, München, DTV, 1978.

27 Simon Williams, Wagner and the Romantic Hero, Cambridge, Cambridge University Press, 2004.

${ }_{28}$ BORCHMEYER, Richard Wagner: Theory and Theatre cit.; ID., Drama and the World of Richard Wagner, Princeton, Princeton University Press, 2003 (trad. di Richard Wagner. Ahasvers Wandlungen, Frankfurt/Leipzig, Insel, 2002).

${ }^{29}$ Francesco Orlando, Proposte per una semantica del Leit-Motiv nell' "Anello del Nibelungo", "Nuova Rivista Musicale Italiana» X, 1975, pp. 230-247; LuCA ZoppelLI, «Der Ring des Nibelungen»: proposta per una lettura narratologica dell'epos wagneriano, «Studi musicali» XX, 1991, pp. 317-338; cfr. anche ID., L'opera come racconto. Modi narrativi nel teatro musicale dell'Ottocento, Venezia, Marsilio, 1994.

${ }^{30}$ EgON Voss, Studien zur Instrumentation Richard Wagners, Regensburg, Bosse, 1970.

${ }^{31}$ Klaus Kropfinger, Wagner and Beethoven. Richard Wagner's reception of Beethoven, Cambridge, Cambridge University Press, 1991.

32 Vedi la nostra introduzione all'edizione del libretto.

33 Tutti i libretti di Wagner, a cura di Olimpio Cescatti, Milano, Garzanti, 19982; le traduzioni di Serpa si possono leggere in Richard Wagner: "Tristan und Isolde», Milano, Teatro alla Scala, 2007 (programma di sala).

34 Willem P. Gerritsen, Anthony G. van Melle, Miti e personaggi del Medioevo. Dizionario di storia, letteratura, arte, musica, a cura di Gabriella Agrati e Maria Letizia Magini, Milano, Bruno Mondadori, 1999.

35 Béroul, Il romanzo di Tristano, a cura di Luciana Cocito, Milano, Jaca Book, 1983; THOMAS, Tristano e Isotta, a cura di Fabio Troncarelli, Milano, Garzanti, $1979\left(2000^{8}\right)$. 


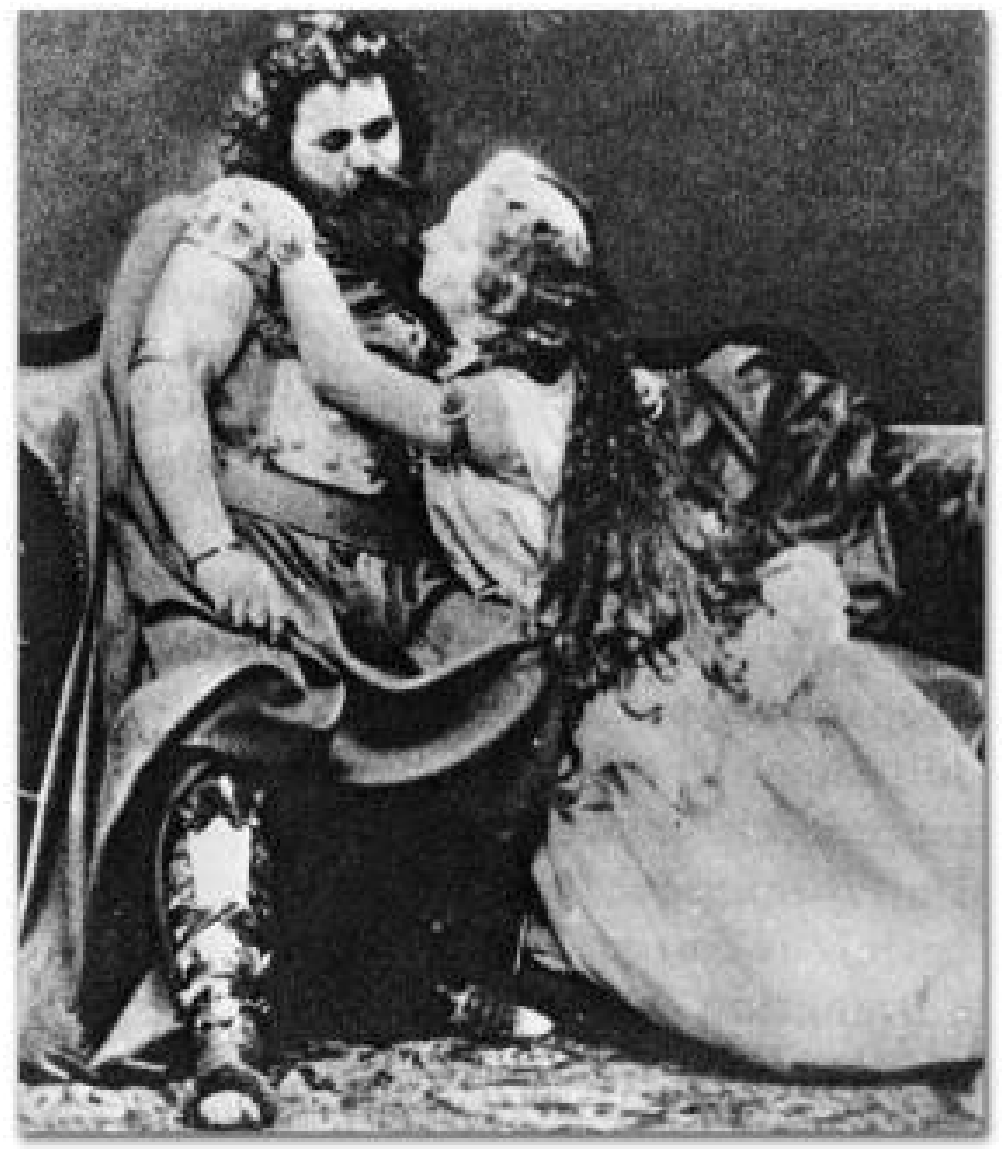

Ludwig Schnorr von Carolsfeld (1836-1865) e la moglie Malwine (Malvina Garrigues; 1825-1904), primi interpreti di Tristan und Isolde al Königliches Hof- und Nationaltheater di Monaco, 1865. Fotografia di Joseph Albert (1825-1886). München, Staatliche Verwaltung der Schlösser.

principio del XIII ${ }^{36}$ e magari con il classico - quanto controverso - rifacimento fin de siècle di Joseph Bédier (Le roman de Tristan et Iseut: «un giardino neogotico orlato di merletti come una porcellana dell'Ottocento"). ${ }^{37}$

Infine, tra le decine di titoli consacrati al Tristan und Isolde, ecco l'elenco dei saggi cui rinvia la guida o che comunque sono stati utili alla sua compilazione, e che potrebbero interessare il lettore: gli studi fin de siècle di Maurice Kufferath, Jean Hubert e Karl Grunsky; ${ }^{38}$ l'analisi dell'ar-

36 Goffredo di StRasburgo, Tristano, a cura di Gabriella Agrati e Maria Letizia Magini, Milano, Mondadori, 1983 (in appendice la traduzione dei frammenti di Thomas).

37 Così l'introduzione di Fabio Troncarelli a THOMAS, Tristano e Isotta cit., p. vIII.

38 Maurice KufFerath, Guide thématique et analyse de «Tristan et Iseult», Paris, Fischbacher, 1894; ID., Tristan et Iseult, Paris, Fischbacher, $1894^{3}$ (trad. it. Tristano e Isotta di Riccardo Wagner. Note e appunti, Torino, Bocca, 1897); JeAn HuBERT, Étude sur quelques pages de Richard Wagner, Paris, Fischbacher, 1895; KARL GRUNSKY, Wagner als Sinfoniker, «Richard Wagner-Jahrbuch», a cura di Ludwig Frankenstein, I, 1906, pp. 227-44. 
monia tristaniana di Ernst Kurth e il discusso ma irrinunciabile Geheimnis der Form di Alfred Lorenz (con il quale si misura praticamente ogni nota del nostro lavoro);39 l'ampia messe di saggi filologici e critici - sul cosiddetto Vorspiel und Isoldens Liebestod, raccolta in un volume curato negli anni Ottanta da Robert Bailey; ${ }^{40}$ il capitolo tristaniano di Joseph Kerman in Opera as Drama e le considerazioni di Carolyn Abbate sulla teoria wagneriana della modulazione; ${ }^{41}$ gli studi sugli schizzi di Ulrich Bartels ${ }^{42}$ e le analisi armoniche di Richard Bass; ${ }^{43}$ le riflessioni di Linda e Michael Hutcheon sul binomio Eros-Thanatos. ${ }^{44}$ Tra le pubblicazioni del nuovo millennio, infine, la monografia di Eric Chafe ${ }^{45}$ e il volume a più mani recentemente curato da Arthur Groos, già autore di un saggio piuttosto noto sul libretto del Tristan. ${ }^{46}$

Su tutt'altro fronte, il bimestrale francese "L'Avant-Scène Opéra» ci offre una vasta disco- e videografia aggiornata al 2011, una selezione di allestimenti e qualche interessante intervista a interpreti tristaniani del calibro di Birgit Nilsson, Jon Vickers, Waltraud Meier. ${ }^{47}$

Il lettore curioso non dovrebbe neppure perdere d'occhio (e d'orecchio) i Wesendonck-Lieder, che - come abbiamo ricordato - intrattengono relazioni significative con il Tristan und Isolde. ${ }^{48}$ Così come potrebbe trovare stimolante il libro di Adriana Guarnieri sulla ricezione di Wagner e del Tristan nella cultura italiana. ${ }^{49}$ Nella sua ricognizione dal 1860 fin dentro al Novecento, la studiosa ha la possibilità di stilare un bilancio decisamente corposo: speriamo lo sia altrettanto quello del secolo appena iniziato.

39 ERnST KuRTH, Romantische Harmonik und ihre Krise in Wagners "Tristan», Berlin, Max Hesses Verlag, 192333 Alfred LoRENZ, Das Geheimnis der Form bei Richard Wagner, 4 voll., II: Der musikalische Aufban von Richard Wagners «Tristan und Isolde», Berlin, Max Hesse, 1926 (rist. Tutzing, Schneider, 1966). Cfr. anche ID., Die formale Gestaltung des Vorspiels zu «Tristan und Isolde», "Zeitschrift für Musikwissenschaft», 5, 1922-1923, pp. 546-557 (ripubblicato in Zur musikalischen Analyse, a cura di Gerhard Schuhmacher, Darmstadt, Wissenschaftliche Buchgesellschaft, 1974, pp. 455-474).

${ }^{40}$ Richard Wagner, Prelude and Transfiguration from «Tristan and Isolde», a cura di Robert Bailey, New York, Norton, 1985.

41 Joseph Kerman, L'opera come dramma, Torino, Einaudi, 1990 (ed. orig. 1988²); CarolYn AbBate, Wagner, "On modulation» and «Tristan», "Cambridge Opera Journal», I/1, 1989, pp. 33-58.

42 UlRICH BARTELS, Analytisch-entstehungsgeschichtliche Studien zu Wagners «Tristan und Isolde» anhand der Kompositionsskizze des zweiten und dritten Aktes, Köln, Studio, [1995].

43 RichaRd BASS, From Gretchen to Tristan: The Changing Role of Harmonic Sequences in the Nineteenth Century, "19 $9^{\text {th }}$ Century Music», XIX/3, 1996, pp. 263-85.

44 Linda HutCheON e Michael HutcheOn, Death drive: Eros and Thanatos in Wagner's «Tristan und Isolde», Cambridge Opera Journal», XI/3, 1999, pp. 267-94.

${ }_{45}$ ERIC CHAFE, The Tragic and the Ecstatic: The Musical Revolution of Wagner's «Tristan und Isolde», Oxford, Oxford University Press, 2005.

${ }_{46}$ Richard Wagner: «Tristan und Isolde», a cura di Arthur Groos, Cambridge, Cambridge University Press, 2011; ARTHUR GROOS, Wagner's "Tristan und Isolde»: in Defence of the Libretto, «Music and Letters», vI/4, 1988, pp. 465-481.

47 «L'Avant-Scène Opéra», 34-35, $2011^{2}$ (acquistabile anche in formato PDF: http://www.asopera.com/fr/).

48 MARTIN STAEHELIN, Von den «Wesendonck-Liedern» zum «Tristan», in Zu Richard Wagner. Acht Bonner Beiträge im Jubiläumsjabr 1983, a cura di Helmut Loos e Günther Massenkeil, Bonn, Bouvier, 1984, pp. 45-73; KLAUS KÖRNER, Richard Wagner: die «Tristan»-Studien: «Im Treibhaus» und «Träume». Wege im Schweizer Exil in eine zukunftsweisende Klangwelt, Köln, Tonger, 1998.

49 Adriana Guarnieri Corazzol, Tristano, mio Tristano. Gli scrittori italiani e il caso Wagner, Bologna, Il Mulino, 1988. 


\title{
Dall'archivio storico del Teatro La Fenice
}

\author{
a cura di Franco Rossi
}

\author{
La prima volta alla Fenice di Antonio Guarnieri
}

Stamani alle 5:21 negli strumenti dell'Osservatorio è incominciata una impressionante, straordinaria registrazione: le ampiezze dei tracciati sono state così grandi che non sono entrate nei cilindri: misurano oltre 40 centimetri. Da qualche parte sta succedendo qualcosa di grave.

Con queste parole gli addetti all'Osservatorio Ximeniano di Firenze registrano all'alba del 28 dicembre 1908 l'eccezionale scossa che aveva devastato le città di Messina e di Reggio Calabria. Sommato agli incendi dovuti alle fughe di gas e alle tre ondate di maremoto che devastarono la costa, il terremoto distrusse il novanta per cento degli edifici messinesi. Il 27 dicembre la città aveva trascorso una serata tranquilla, impegnata nella celebrazione della festa di Santa Barbara; proprio per arricchire le festività, a teatro era stata allestita la prima di Aida; il grande successo del tenore Angelo Gamba, ovviamente Radames, fu pagato a carissimo prezzo, dal momento che sparì sotto le macerie dell'Hotel Europa, insieme alla moglie e ai due figli.

È in questo stato d'animo che si svolge alla Fenice la stagione di carnevale del 1908-1909, che offre un cartellone di notevole interesse: accanto a Eidelberga mia! di Ubaldo Pacchierotti, appaiono per la prima volta a Venezia Madama Butterfly di Puccini e Tristano e Isotta di Wagner. A completare la stagione una delle numerose riprese di Aida, allestita per la quarta volta alla Fenice. La presidenza dell'assemblea dei soci è affidata a Giacomo Levi, mentre il direttore agli spettacoli è Marco Sullam; a completare l'insieme della dirigenza Pierluigi Zannini, Augusto Mayneri (direttore anziano) e Giuseppe Lazzari (direttore cassiere), mentre l'impresa è affidata a Pasquale Zeni di Montagnana, per la prima e unica volta alla guida della Fenice, nonostante il buon esito della stagione. ${ }^{1}$

Come di norma, anche in questa stagione l'appalto prevede una gara tra impresari diversi. Tra questi troviamo Enrico Corti, già appaltatore nella stagione 1904-1905 (con Sigfrido, Germania di Franchetti, La traviata e Otello), che propone una stagione non dissimile da quella che poi vedrà la luce, salvo un Battista in luogo di Eidelberga mia!, e che per il cast vocale impone una clausola secondo la quale «il soprano e il baritono per la Butterfly verranno concordati colla casa Ricordi la quale si riserva il diritto di scegliere gli artisti che ritiene più convenienti». ${ }^{2}$ Anche l'impresa Cambiaggio manifesta interesse per la stagione: accanto alle tre opere che vedranno la luce propone al posto della novità Eidelberga mia! una più sicura Gioconda. Ma anche qui le spese a carico del teatro (fra cui il direttore d'orchestra) sembrano decisamente troppe. Poiché l'impresario Crestani aveva nel frattempo firmato l'accordo con Trieste, a Venezia non resta dunque

\footnotetext{
${ }^{1}$ In questi anni molto difficilmente gli impresari possono contare su un reincarico, anche al di là dell'esito favorevole delle stagioni da loro gestite: il loro avvicendamento è prassi comune.

2 Lettera da Milano, in data 21 aprile 1908. Archivio storico del Teatro La Fenice, buste Spettacoli, n. 484, da cui provengono anche i documenti citati poco oltre.
} 


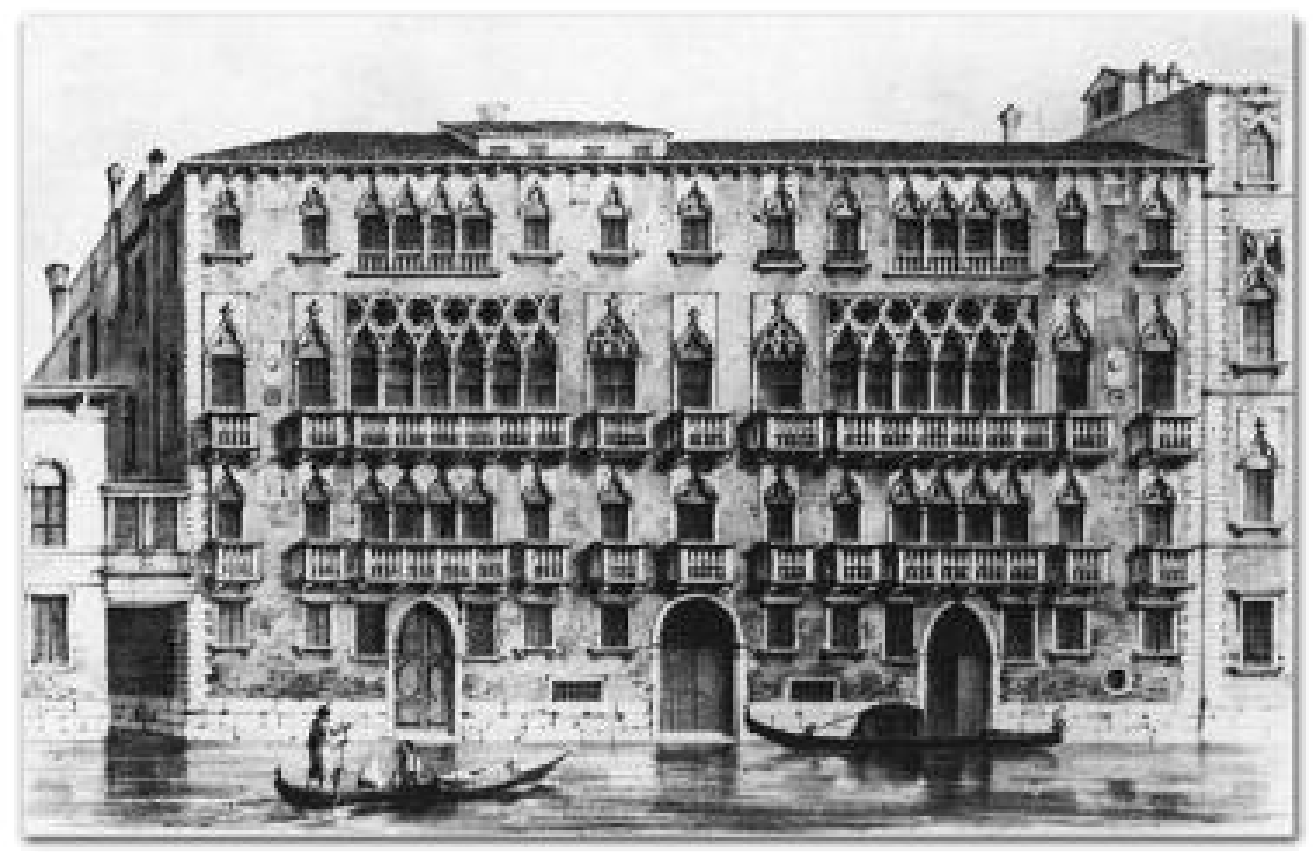

Palazzo Giustinian Brandolini d'Adda, prima abitazione veneziana di Wagner. Tra il settembre 1858 e il marzo 1859 Wagner compose in questo palazzo il secondo atto di Tristan und Isolde. Incisione di Marco Moro, 1846.

che Pasquale Zeni: dopo estenuanti trattative nelle quali si inserisce spesso anche la casa editrice Ricordi, sempre più parte attiva in molte gestioni teatrali, e dopo aver accarezzato l'ipotesi di produrre un più facile Elisir d'amore, si giunge con qualche mugugno alla formalizzazione dell'appalto, che viene siglato il 15 dicembre, undici giorni prima dell'alzata del sipario.

Zeni propone una compagnia di canto formata dai soprani Cecilia David e Nina Tezza Gallo, dal mezzo Ladislava Hotkowska, dai tenori David Henderson e Angelo Bendinelli, dai baritoni Bellantoni e Federici e dal basso Carrozzi; oramai nei contratti vengono indicati anche ruoli che anni prima non avrebbero goduto degli onori delle cronache: il direttore d'orchestra veneziano Antonio Guarnieri (con il sostituto vicentino Arrigo Pedrollo) e il maestro di coro Vittore Veneziani. Interessante è anche la lettura dei contratti siglati con gli artisti e dei relativi compensi: Ladislava Hotkowska percepirà 5.250 lire per interpretare tre opere su quattro (in particolare Aida e Tristano e Isotta), Giuseppina Piccoletti 4.000 lire per Madama Butterfly e Eidelberga mia!, David Henderson (anch'egli per Aida e Tristano e Isotta) arriverà a 11.500 lire, Giuseppe Bellantoni (tre opere su quattro) ne ricaverà 6.500 lire, Angelo Bendinelli (due opere su quattro) 8.000 lire. Sono cifre significative, tanto più se vi sommiamo i compensi dei comprimari; non è difficile allora comprendere come spesso si possa giungere a stagioni economicamente traballanti.

La stagione andò comunque abbastanza bene: trentasette recite complessive di cui sedici di Aida, nove di Tristano e Isotta, otto di Madama Butterfly e quattro di Eidelberga mia!. In linea con questi risultati gli incassi: $35.804,35$ lire per Aida (con una media di 2.238 lire a recita), 16.132,85 per Tristano e Isotta (1.793 a serata), solo 9.909,95 per Madama Butterfly (1.239 lire) e 5.171,6 
lire per Eidelberga mia! (con una media di 1.293 lire a sera). Se era facilmente prevedibile il successo di Aida (ed è abbastanza naturale considerare che la media degli incassi resti comunque la più elevata), meno lo era quello di Tristano e Isotta; e parallelamente, se prevedibile era il modesto esito del lavoro nuovo (che non fu peraltro, come annunciato, una prima assoluta), qualche perplessità suscita la tiepida ricezione di Madama Butterfly: il ricordo ancor vivo del fiasco della première (1904) aveva forse reso diffidenti anche i veneziani, e se otto recite non sono poche, l'esiguità dell'incasso stupisce un poco, sia noi sia, probabilmente, l'impresa: il felice esito iniziale, poi smentito dalle riprese, aveva portato l'ente a felicitarsi con editore e compositore, e l'archivio conserva gelosamente il telegramma di congratulazioni di quest'ultimo: «Ringrazio vivamente per gentile comunicazione successo Butterfly. Prego complimentare maestro Guarnieri valenti artisti ossequi. Puccini».

La presentazione di Tristano e Isotta (la traduzione italiana era di prassi allora) è affidata, come di consueto, a un ampio articolo pubblicato il giorno stesso della prima. Non solo troviamo trama e commenti entusiasti dell'evento, ma addirittura una breve cronologia della tradizione esecutiva dell'opera:

Il 10 giugno 1865, a Monaco, l'opera alla sua prima comparsa ebbe quel che oggi si direbbe un successo di stima, inferiore a quello della trilogia che l'argomento rendeva d'un subito popolare. I tempi e il pubblico non eran maturi - e pure non si trattava del tanto bistrattato pubblico italiano, al quale non si può neanche rimproverare lo snobismo, perché gliel'hanno imposto a forza di dargli dell'ignorante! L'opera ebbe tardo e lento il cammino: alcune date lo dimostrano. Prima ripresa di poche recite a Monaco nel 1869, seconda ripresa e questa decisiva nel 1872. Poi il cammino si fa più rapido: Weimar (1874), Londra (1882), Vienna (1883), Parigi (nei concerti Lamoureux 1884-85), Bologna (1888), Bruxelles (1894), Torino (1894), Parigi (Opéra 1895). [...] Arturo Toscanini, dopo aver diretto Tristano a Torino (1894), lo riprese nel 1900 alla Scala, e da quell'anno i maggiori teatri d'Italia gareggiano nel riprodurlo: Roma 1903 (Mancinelli), Ravenna 1904 (Vanzo), Scala 1906, Brescia 1906, Bologna e Trieste 1907, Napoli, Parma e Genova 1908. E Venezia 1909. Accontentiamoci. ${ }^{3}$

La serata inaugurale del 30 gennaio 1909 dovette cogliere nel segno, almeno a giudicare dai toni della recensione apparsa poche ore dopo (è proprio il caso di dire, visto che il lavoro si era concluso all'una del mattino):

L'avvenimento artistico si è felicemente compiuto. Tristano $e$ Isotta ha trionfato. Si può ben parlare di trionfo, ché il successo dell'opera fu il più pronto, il più immediato, il più convinto fra quanti ne contarono a Venezia i melodrammi wagneriani. Il teatro era magnifico, quantunque non completamente affollato. Il pubblico era preparato, raccolto, attento in modo tale da fargli veramente onore. E il raccoglimento e l'attenzione furon continui, e continua e vigile la intolleranza di un rumore di ritardatari, di ogni bisbiglio, di ogni colpo di tosse. Un pubblico quale l'opera esige e si merita, un pubblico, per dir tutto in una parola, confortante. [...] Lungamente durò l'applauso finale, e il pubblico tardò a sfollare, malgrado l'opera sia finita all'una precisa, per salutare colla più viva cordialità interpreti e direttore. ${ }^{4}$

$\mathrm{Ma}$ è grazie ai borderò conservati in archivio che è possibile cogliere la sintesi estrema dello spettacolo: non solo vengono indicati gli incassi serali, ma viene addirittura rilevato il tempo atmosferico («nevoso» alla prima, «bello» per tutte le altre recite ad eccezione dell'ultima, dove «tutta la mattinata nevicò»), così come la concorrenza cittadina: ogni sera erano aperti i teatri Rossini, Goldoni e Malibran, o almeno due su tre. In realtà sembra la scelta del giorno della set-

\footnotetext{
3 «La gazzetta di Venezia», 30 gennaio 1909.

4 «Tristano e Isotta» alla Fenice, ivi, 31 gennaio 1909.
} 
timana che influisce maggiormente sul successo. Infatti, oltre all'ovvio prevalere della prima rappresentazione (che incassa il doppio della seconda), i giorni più gettonati sono naturalmente il sabato e la domenica.

Ma qual è la realtà nella quale la Fenice si trova ad operare? A fronte del raffinato programma 'tedesco' della Fenice, il Teatro Rossini ricorre a un successo assicurato:

Per l'ultima rappresentazione di Traviata il Rossini era ieri gremito da cima a fondo di pubblico che fece all'opera e agli esecutori le più cordiali accoglienze.

E la sera successiva è la volta del Trovatore, eseguito «dalle signore Ida Giacomelli [già protagonista della Norma], Flora Perini [che il 27 dicembre cantava al Massimo di Messina ed era scampata per miracolo al disastro], tenore Colazza, baritono Nicoletti». Ma anche nelle serate successive, quando si ricorre alla prosa, il teatro vanta il tutto esaurito. ${ }^{5}$

Ma non è solo il Rossini a concorrere con la Fenice: anche il Goldoni ci mette del suo con commedie di Goldoni, Morais e Ottolenghi ${ }^{6}$, mentre al Malibran va in scena l'operetta Primavera scapigliata su musiche di Josef Strauss arrangiate da Ernst Reiterer. ${ }^{7}$

A maggior ragione dobbiamo quindi tenere nella più alta considerazione la felice accoglienza che Venezia riserva a Tristano e Isotta: alla seconda rappresentazione il teatro non sembra essere troppo affollato, ma

l'esecuzione fu eccellente, e i cinque interpreti principali, la signore Grisi e Hotkowska, i signori Henderson, Bellantoni e Carrozzi, si confermarono degni di ogni maggior elogio per voce, per azione, per severo senso d'arte. Il Guarnieri diresse splendidamente e l'orchestra lo seguì - così ci parve - più animata ed energica dell'altra sera, risentendo l'effetto benefico di un po' di riposo.

Veneziano, violoncellista, l'allora ventinovenne Guarnieri aveva iniziato cinque anni prima a Siena la sua carriera di direttore ed era reduce da un memorabile Tristano alla Hofoper di Vienna, di cui nel 1912 sarebbe stato nominato direttore principale. Si sarebbe presto affermato come uno dei maggiori direttori italiani contemporanei di Toscanini, insieme rigoroso e curioso del nuovo, con una predilezione particolare per il repertorio wagneriano. Con Aida, Butterfly, Tristano ed Eidelberga debuttava alla Fenice. Vi sarebbe tornato tante altre volte, e nel 1940 di nuovo con Tristano.

\footnotetext{
5 «Stasera per la prima volta a Venezia Giovanni Grasso interpreta Pietra fra pietre di Ermanno Sudermann, appositamente ridotta pel teatro siciliano", ibidem.

6 «La signora Ester Gelich Sainati fu iersera molto applaudita nella recita, che era in suo onore, della Casa nova e dell'annunciato monologo. [...] Questa sera avremo la penultima recita della stagione con un programma sempre bene accetto: L'avvocato difensor di Mario Morais e In pretura di Giuseppe Ottolenghi», ivi, 30 gennaio 1909.

7 «Iersera la signorina Roma Criscuolo ha avuto con Primavera scapigliata la sua serata d'onore. Vide un teatro affollatissimo e riscosse applausi interminabili. La graziosa attrice dopo il secondo atto cantò alcune canzonette premiate all'ultimo concorso di Piedigrotta con grande successo", ivi.
} 


\section{Tristan und Isolde / Tristano e Isotta al Teatro La Fenice.}

Handlung in drei Aufzügen / dramma in tre atti di Richard Wagner; ordine dei personaggi: 1. Tristan 2. Re Marke 3. Isolde 4. Kurwenal 5. Melot 6. Brangäne 7. Un pastore 8. Un pilota 9. Un giovane marinaio.

\section{8-1909 - Stagione di carnevale}

30 gennaio 1909 (9 recite, nella trad. italiana di Pietro Floridia).

1. David Henderson 2. Oreste Carrozzi 3. Maria Grisi 4. Giuseppe Bellantoni 5. Palmiro Domenichetti 6. Ladislava Hotkowska 8. Baldassarre Benedetti - $\mathrm{M}^{\circ}$ conc.: Antonio Guarnieri; $\mathrm{m}^{\circ}$ del coro: Vittore Veneziani; dir. scena: Cesare Razzani.

\section{2-1923 - Stagione di carnevale}

26 dicembre 1922 (9 recite, nella trad. italiana di Pietro Floridia).

1. Luigi Canalda 2. Vittorio Julio 3. Sara Cesar 4. Giuseppe Noto 5. Attilio Muzio 6. Giannina Arangi Lombardi 7. Gino Treves 8. Angelo Zoni 9. Gino Treves - $\mathrm{M}^{\circ}$ conc.: Giuseppe Baroni (Mario Bellini); $\mathrm{m}^{\circ}$ del coro: Ferruccio Cusinati; dir. scena: Mario Villa.

\section{0 - Stagione lirico-sinfonica dell'anno XVIII}

14 febbraio 1940 (4 recite, nella trad. italiana di Pietro Floridia).

1. Giovanni Voyer 2. Andrea Mongelli 3. Ella De Nemethy 4. Antenore Reali 5. Piero Zennaro 6. Gilda Alfano 7. Luigi Nardi 8. Ubaldo Corelli 9. Mario Tinti - $\mathrm{M}^{\circ}$ conc.: Antonio Guarnieri; $\mathrm{m}^{\circ}$ coro: Sante Zanon; reg.: Carlo Piccinato; scen.: Antonio Rovescalli (proprietà Teatro alla Scala); cost.: Casa d'arte Caramba.

\section{2 - Manifestazioni musicali dell'anno XX}

4 aprile 1942 (2 recite, in lingua originale).

1. Julius Pölzer 2. Josef Greindl 3. Margarete Bäumer 4. Rudolf Grossmann 5. Erik Bräuer 6. Res Fischer 7. Alfred Pfeifle $-\mathrm{M}^{\circ}$ conc.: Hugo Balzer; $\mathrm{m}^{\circ}$ coro: Sante Zanon; reg.: Georg Hartmann; scen. e cost.: Baldo Giuberti.

\section{7-1948 - Stagione lirica di carnevale}

30 dicembre 1947 (4 recite, nella trad. italiana di Pietro Floridia).

1. Fiorenzo Tasso 2. Boris Christoff 3. Maria Callas 4. Raimondo Torres 5. Attilio Barbesi 6. Fedora Barbieri 7. Guglielmo Torcoli 8. Uberto Scaglione 9. Ottorino Begali - $\mathrm{M}^{\circ}$ conc.: Tullio Serafin; $\mathrm{M}^{\circ}$ del coro: Sante Zanon; $\mathrm{m}^{\circ}$ banda: Alfredo Ceccherini; reg.: Mario Frigerio; scen.: Ercole Sormani; cost.: Casa d'arte Cerratelli.

\section{2-1953 - Stagione lirica di carnevale}

16 maggio 1953 (3 recite, in lingua originale).*

1. Wolfgang Windgassen 2. Wilhelm Schirp 3. Martha Mödl 4. Gustav Neidlinger 5. Gustav Grefe 6. Res Fischer (Ira Malaniuk) 7. Alfred Pfeifle 8. Giorgio Santi 9. Alfred Pfeifle $-\mathrm{M}^{\circ}$ conc.: Ferdinand Leitner; $\mathrm{m}^{\circ}$ coro: Sante Zanon; reg.: Heinz Arnold; scen. e cost.: Helmut Kontarsky; complesso artistico dello Staatstheater di Stoccarda (artisti di canto del Teatro di Bayreuth); Orchestra e Coro del Teatro La Fenice.

* Le recite celebravano il $70^{\circ}$ anniversario della morte di Richard Wagner.

\section{7-1958 - Stagione lirica invernale}

13 febbraio 1958 (3 recite, in lingua originale). **

1. Wolfgang Windgassen 2. Josef Greindl 3. Birgit Nilsson 4. Gustav Neidlinger 5. Fritz Uhl 6. Grace Hoffman 
7. Hermann Winkler 8. Hans Habietinek 9. Fritz Uhl - $\mathrm{M}^{\circ}$ conc.: Wolfgang Sawallisch; $\mathrm{m}^{\circ}$ coro: Sante Zanon; reg. e scen.: Wolfgang Wagner; cost.: Kurt Palm; Orchestra e Coro del Teatro La Fenice; allestimento dei Bayreuther Festspiele 1957.

$\because$ Le recite celebravano il $75^{\circ}$ anniversario della morte di Richard Wagner.

\section{5-1966 - Stagione lirica invernale}

7 gennaio 1966 (3 recite, in lingua originale).

1. Hans Beirer 2. Frederick Guthrie 3. Elsa Cavelti 4. Gustav Neidlinger 5. Paolo Cesari 6. Ira Malaniuk 7. Augusto Pedroni 8. Giorgio Santi 9. Vittorio Pandano - $\mathrm{M}^{\circ}$ conc.: Karl Maria Zwissler; $\mathrm{m}^{\circ}$ coro: Corrado Mirandola; reg.: Peter Lehmann; scen.: Sormani; cost.: Casa d'arte Fiore.

\section{0-1971 - Stagione lirica}

31 marzo 1971 (5 recite).

1. Wolfgang Windgassen (Hermin Esser) 2. Eduard Wollitz 3. Gunilla af Malmborg 4. Hans Günter Nöcker 5. Heinz Günter Zimmermann 6. Grace Hoffman 7. Heribert Steinbach 8. Marco Stefanoni 9. Heribert Steinbach - $\mathrm{M}^{\circ}$ conc.: Kurt Masur; $\mathrm{m}^{\circ}$ coro: Corrado Mirandola; reg.: Luigi Squarzina; scen. e cost.: Giacomo Manzù; nuovo all.

\section{1 - Stagione d'opera e teatro musicale}

29 gennaio 1981 (7 recite, in lingua originale).

1. Hermin Esser (Heribert Steinbach) 2. Kurt Rydl 3. Johanna Meier (Dagmar Trabert) 4. Leif Roar (Heinz Jürgen Demitz) 5. Heribert Steinbach (Jörn W. Wilsing) 6. Ruthild Engert 7. Pio Bonfanti (Antonio Balbo) 8. Guido Fabbris 9. Giuseppe Botta (Antonio Balbo) - $\mathrm{M}^{\circ}$ conc.: Peter Maag; $\mathrm{m}^{\circ}$ coro: Aldo Danieli; reg.: Maria Francesca Siciliani; scen. e cost.: Alberto Burri; all.: Teatro Regio di Torino.

\section{3-1994 - Stagione di lirica e balletto}

16 aprile 1994 (7 recite, in lingua originale)

1. Siegfried Jerusalem (Wolfgang Fassler) 2. Hans Sotin 3. Gabriele Schnaut (Ingrid Hauboldt) 4. Hartmut Welker 5. Reiner Buese 6. Hanna Schwarz (Cornelia Wulkopf) 7. Fabio Sartori 8. Giuseppe Zecchillo 9. Jurgen Schaefer $-\mathrm{M}^{\circ}$ conc.: Marek Janowski (Hans Hilsdorf); $\mathrm{m}^{\circ}$ coro: Giovanni Andreoli; prod. orig. Michael Hampe; reg.: Florian Malte Leibrecht; scen. e cost.: Mauro Pagano; all.: Oper der Stadt Köln.

\section{2 - Stagione di lirica e balletto. Venezia, Teatro Malibran}

22 giugno 2002 (5 recite, in lingua originale) $* * * *$

1. Siegfried Jerusalem (Raimo Sirkiä) 2. Matti Salminen 3. Eva Johansson 4. Peter Weber 5. Christer Bladin 6. Doris Soffel 7. Daisuke Sakaki 8. Franco Boscolo 9. Christer Bladin - $\mathrm{M}^{\circ}$ conc.: Isaac Karabtchevsky; $\mathrm{m}^{\circ}$ coro: Guillaume Tourniaire.

*** L'opera è stata data in forma di concerto 


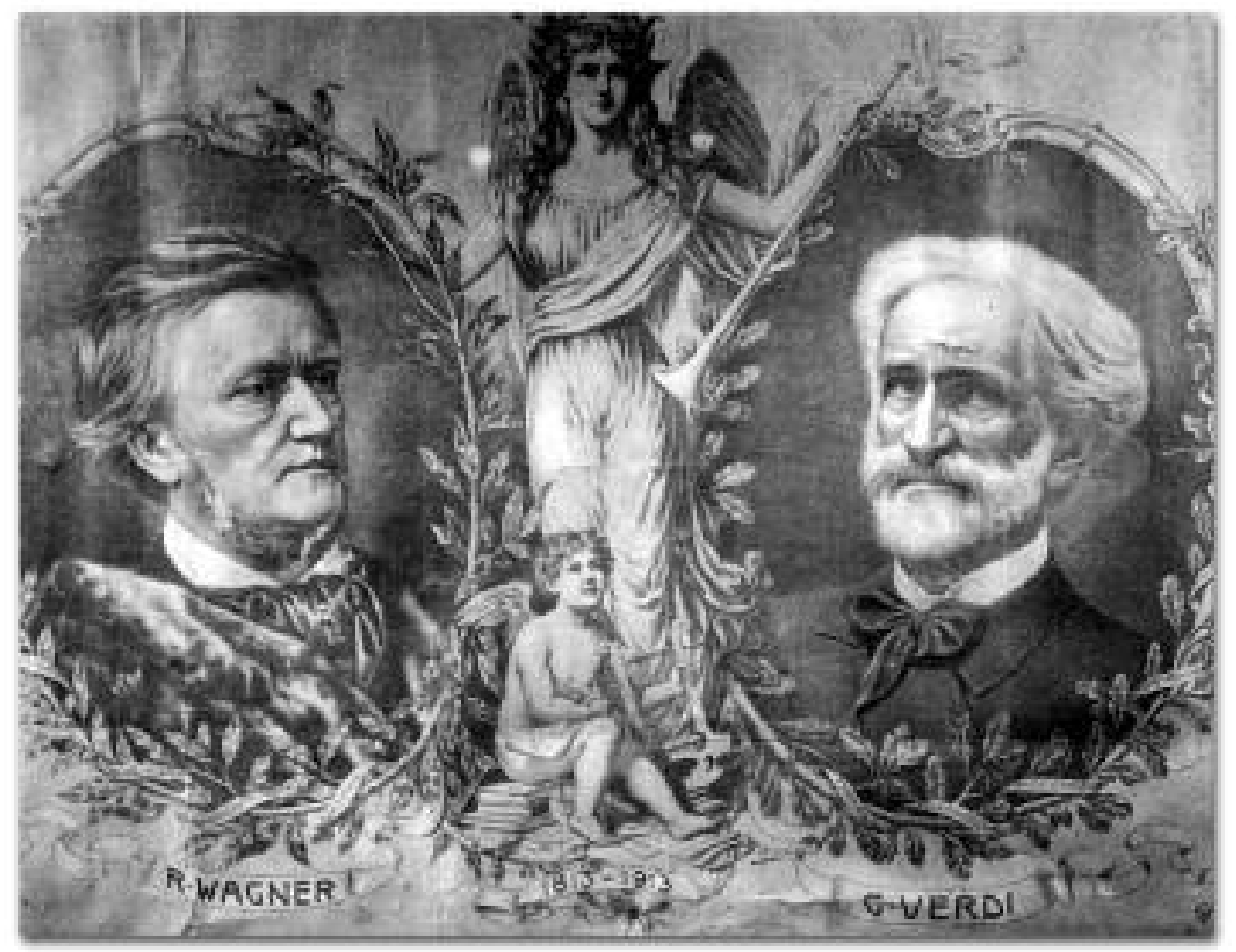

O. PASCIONI, Richard Wagner e Giuseppe Verdi (nel primo centenario della nascita). Litografia, 1913. 


\section{Biografie}

\section{MYUNG-Whun Chung}

Maestro concertatore e direttore d'orchestra. Nato a Seoul in Corea, inizia l'attività musicale come pianista debuttando all'età di sette anni, e a ventuno vince il secondo premio al Concorso Pianistico Čajkovskij di Mosca. Si perfeziona al Mannes College e alla Juilliard School di New York e nel 1979 diviene assistente di Carlo Maria Giulini alla Los Angeles Philharmonic, di cui nel 1981 è nominato direttore associato. Dal 1984 al 1990 è direttore musicale dell'Orchestra Sinfonica della Radio di Saarbrücken, dal 1987 al 1992 direttore ospite principale del Teatro Comunale di Firenze, dal 1989 al 1994 direttore musicale dell'Orchestra dell'Opéra di Parigi, dal 1997 al 2005 direttore principale dell'Orchestra dell'Accademia di Santa Cecilia. Nel 1995 fonda la Asia Philharmonic, e nel 2005 è nominato direttore principale della Seoul Philharmonic Orchestra. Dal 2000 è direttore musicale dell'Orchestre Philharmonique de Radio France e nel 2011 è stato nominato direttore ospite principale della Staatskapelle Dresden. Ha diretto le più prestigiose orchestre europee e statunitensi, fra cui Berliner e Wiener Philharmoniker, Concertgebouw di Amsterdam, le principali orchestre di Londra e Parigi, Orchestra Filarmonica della Scala, Bayerischer Rundfunk, Dresden Staatskapelle, Orchestra del Metropolitan di New York, New York Philharmonic, orchestre di Cleveland, Philadelphia, Boston e Chicago. Ha ricevuto numerosi riconoscimenti tra cui in Italia il Premio Abbiati e il Premio Toscanini e in Francia la Légion d'Honneur (1992), la nomina ad Artista dell'anno dal Sindacato della critica drammatica e musicale (1991), il premio Victoires de la Musique (1995 e 2002) e la nomina a Commandeur dans l'ordre des Arts et des Lettres (2011). Parallelamente alla sua attività musicale, è impegnato in iniziative di carattere umanitario e di diffusione della musica classica tra le giovani generazioni, nonché di salvaguardia dell'ambiente. Ambasciatore del programma delle Nazioni Unite per il controllo internazionale della droga (UNDCP), nel 1995 è stato nominato Uomo dell'anno dall'UNESCO e nel 1996 il Governo della Corea del Sud gli ha conferito il Kumkuan, il più importante riconoscimento coreano in campo culturale. $\grave{\mathrm{E}}$ attualmente Ambasciatore onorario per la cultura della Corea e dal 2008 è Ambasciatore di buona volontà dell'UNICEF. Nel 2012 è riuscito a riunire per la prima volta alla Salle Pleyel di Parigi la Unhasu Orchestra della Corea del Nord e l'Orchestre Philharmonique de Radio France.

\section{Paul CuRRan}

Regista. Nato a Glasgow in Scozia e diplomato in regia teatrale presso il National Institute of Dramatic Art di Sydney e presso la Suomen Kansallisooppera di Helsinki, è stato direttore artistico della Norske Opera di Oslo dal 2007 al 2011. Ha lavorato nei principali teatri europei (Covent Garden, Welsh National Opera, Garsington Opera, Festival di Wexford, Scala, Opera di Roma, Napoli, Firenze, Venezia, Bologna, Cagliari, Trieste, Vlaamse Opera di Anversa, Staats- 
theater di Stoccarda, São Carlos di Lisbona, Teatro Mariinskij di San Pietroburgo) e internazionali (Lyric Opera di Chicago, Santa Fe Opera, Washington Opera, Spoleto Festival USA, Disney Hall di Los Angeles, Canadian Opera, Bunka Kaikan di Tokyo) mettendo in scena lavori di Monteverdi (L'incoronazione di Poppea), Cavalli (La Statira), Mozart (La finta giardiniera, Die Zauberflöte), Rossini (La Cenerentola), Bellini (I Capuleti e i Montecchi), Verdi (I lombardi alla prima crociata, Rigoletto, Il trovatore, Otello), Puccini (La bohème, Tosca), Wolf-Ferrari (I gioielli della Madonna), Massenet (Chérubin), Thomas (Hamlet), Wagner (Tannhäuser, Tristan und Isolde), Johann Strauss (Die Fledermaus), Humperdinck (Königskinder, Premio Abbiati 2003), Richard Strauss (Die Frau obne Schatten, Ariadne auf Naxos, Daphne), Zemlinsky (Eine florentinische Tragödie), Berg (Lulu), Borodin (Il principe Igor), Rimskij-Korsakov (La fidanzata dello zar), Rachmaninov (Il cavaliere avaro), Šostakovič (Una Lady Macbeth del distretto di Mcensk), Foerster (Eva), Martinů (Mirandolina), Britten (Billy Budd, Albert Herring, Peter Grimes, The Rape of Lucretia, A Midsummer Night's Dream), Sondheim (A Funny Thing Happened on the Way to the Forum, A Little Night Music), Leigh (Man of La Mancha), Pesson (la prima assoluta di Pastorale), Sallinen (Kullervo). Tra gli impegni della stagione 2011-2012 ricordiamo Die Zauberflöte a Oslo, Rusalka a Tokyo, Pagliacci a Copenaghen, Tosca a Toronto, Albert Herring a Los Angeles, A Midsummer Night's Dream all'Opera di Roma, Peter Grimes a Savonlinna. Per il Teatro La Fenice ha firmato le regie di Ariadne auf Naxos nel 2003 e Daphne nel 2005.

\section{ROBERT INNES HOPKINS}

Scenografo e costumista. Premiato nel 2007 come «Bühnenbildner des Jahres» dalla rivista Opernwelt (per Die Soldaten alla Ruhrtriennale) e vincitore del Critics Circle Designer of the Year Award 1996 (per The Comedy of Errors con la Royal Shakespeare Company e The Weavers a Dublino) e di due TMA Designer of the Year Awards (nel 1996 per The Wasp Factory a Leeds e My Mother Said con la Oxford Shakespeare Company e nel 2007 per Promises Promises a Sheffield), è attivo tanto nel teatro lirico quanto in quello di prosa. In ambito lirico ha firmato scene e costumi di lavori di Händel (Serse), Rossini (L'italiana in Algeri), Bellini (Norma, I Capuleti e i Montecchi), Donizetti (L'elisir d'amore), Verdi (Rigoletto, Simon Boccanegra), Puccini (Manon Lescaut, La bohème), Mascagni (Cavalleria rusticana), Leoncavallo (Pagliacci), Bizet (Carmen), Borodin (Il principe Igor), Cajkovskij (La maliarda), Prokof'ev (Matrimonio al convento), Šstakovič (Mosca quartiere Čerëmuški), Smetana (La sposa venduta), Janáček (La volpe astuta), Wagner (Lohengrin, Der fliegende Holländer, Tristan und Isolde, Parsifal), Berg (Wozzeck), Zimmermann (Die Soldaten), Britten (Billy Budd, Peter Grimes, The Rape of Lucretia), Maxwell Davies (Kommilitonen), O'Regan (Heart of Darkness) nei principali teatri inglesi (Linbury, Royal Academy of Music, Glyndebourne, Aldeburgh, Opera North, Welsh National Opera, Grange Park Opera) e internazionali (Berlino, Amburgo, Ruhrtriennale, Zurigo, Ginevra, Bregenz, Oslo, Stoccolma, Strasburgo, Valencia, Oviedo, Lisbona, Tallinn, Mosca, San Pietroburgo, San Francisco, Chicago, New York, Houston, Washington, Santa Fe, Nuova Zelanda). Nell'ambito del teatro di prosa ha lavorato nei principali teatri britannici a testi che vanno da Shakespeare a Brecht e Hauptmann ai più recenti autori contemporanei inglesi.

\section{IAN STOREY}

Tenore, interprete del ruolo di Tristan. Nato in Inghilterra, studia disegno e grafica all'Università di Loughborough, trasferendosi poi in Nuova Zelanda dove inizia lo studio del canto. Prosegue gli studi a Londra con Laura Sarti e in Italia con Carlo Cossutta. Debutta nel 1991 in The Rape of Lucretia di Britten, La bohème e Aida e nel 1993 prende parte ai corsi del National Ope- 
ra Studio di Londra, esibendosi in seguito in numerose produzioni della Scottish Opera di Glasgow e della Welsh National Opera di Cardiff. Nel 1997 debutta in Italia in Turandot a Torre del Lago, iniziando un'importante carriera internazionale che l'ha portato ad esibirsi nei principali teatri europei (Scala, San Carlo di Napoli, Comunale di Bologna, Fenice di Venezia, Arena di Verona, Cagliari, Palermo, Genova, Glyndebourne, Amsterdam, Madrid, Barcellona, Lisbona, Montpellier, Zurigo, San Gallo, Bregenz, Staatsoper e Deutsche Oper di Berlino, Francoforte, Düsseldorf, Riga) e americani (Los Angeles, Washington, San Francisco, São Paulo). Il repertorio debuttato comprende lavori di Beethoven (Fidelio), Verdi (Il trovatore, Un ballo in maschera, La forza del destino, Otello), Puccini (La bohème, Tosca, Madama Butterfly, La fanciulla del West, Turandot), Mascagni (Cavalleria rusticana), Smareglia (Nozze istriane), Berlioz (Les troyens), Bizet (Carmen), Saint-Saëns (Samson et Dalila), Wagner (Tannhäuser, Der fliegende Holländer, Tristan und Isolde), Strauss (Ariadne auf Naxos, Elektra), Berg (Lulu), Musorgskij (Boris Godunov), Čajkovskij (La dama di picche), Sostakovič (Una Lady Macbeth del distretto di Mcensk), Janáček (Věc Makropulos, Kát’a Kabanová, Jenưfa). Ha collaborato con direttori quali Barenboim, Chailly, Gatti, Inbal, Metzmacher, Plasson, Armstrong, Jurowski, Rizzi.

\section{ATtILA Jun}

Basso, interprete del ruolo di re Marke. Nato a Seoul in Corea, inizia gli studi all'Università di Seoul con Philip Kang e a 21 anni debutta in ruoli quali Sarastro, Filippo II, Colline e Daland. Dopo aver vinto tre concorsi in Corea ed essere stato premiato ai concorsi Belvedere di Vienna e Re Manfredi di Manfredonia si trasferisce nel 1998 in Germania per completare gli studi con Hans Sotin alla Musikhochschule di Colonia. Debutta in Europa nel 1998 alla Staatsoper di Stoccarda, della cui compagnia fa parte dal 1999. Oltre che a Stoccarda, si è esibito nei principali teatri e festival europei (Bayreuth, Deutsche Oper di Berlino, Amburgo, Wels, Staatsoper di Vienna, Salisburgo, Amsterdam, Anversa, Copenaghen, Manchester, Tolosa, Barcellona, Madrid, La Coruña, Scala di Milano, Opera di Roma, Firenze, Venezia), a San Francisco e Tokyo. Il suo repertorio spazia da Mozart (il Commendatore, Sarastro), Bellini (Oroveso), Verdi (Messa da Requiem) e Puccini (Timur, Messa di Gloria) a Weber (l'eremita nel Freischütz), Wagner (il langravio, Heinrich der Vogler, Daland, Hunding, Hagen, re Marke, il guardiano notturno, Gurnemanz) e Strauss ('Holsteiner in Friedenstag). Ha collaborato con direttori quali Sinopoli, Steinberg, Pappano, Thielemann, Eschenbach, Tate, Minkowski, Dudamel, Lopez-Cobos, Mehta, Elder, Maazel. Nel 2011 ha debuttato il ruolo di Gurnemanz in Parsifal alla Staatsoper di Stoccarda e ha debuttato alla Staatsoper di Vienna come Hagen nella Götterdämmerung; nel 2012 ha debuttato alla Deutsche Oper di Berlino come Hunding nella Walküre e ha cantato Hagen nella Götterdämmerung alla Bayerische Staatsoper di Monaco con Nagano e Ramfis in Aida al Liceu di Barcellona. Nell'aprile 2011 è stato nominato Kammersänger della Staatsoper di Stoccarda. Alla Fenice è stato il Commendatore in Don Giovanni nel 2010 e Fafner nel Rheingold nel 2011.

\section{BRIGITTE PINTER}

Soprano, interprete del ruolo di Isolde. Nata in Austria, debutta alle Wiener Festwochen con Claudio Abbado, che la spinge a perfezionarsi alla Juilliard School di New York. Rientrata in Europa, è tra i vincitori del Concorso Belvedere di Vienna e inizia un'importante carriera internazionale come mezzosoprano che la porta ad esibirsi tra l'altro alla Scala di Milano (Klementia in Sancta Susanna di Hindemith, interpretata anche, con Muti, a Ravenna e New York), all'Opera di Roma (Jocaste in Oedipus Rex), al Massimo di Palermo (Erika in Vanessa di Barber, la Ge- 
schwitz in Lulu di Berg), alla Deutsche Oper di Berlino (Venus in Tannhäuser), ad Amburgo (Ariane et Barbe-Bleue), Francoforte (Ulrica in Un ballo in maschera, Brangäne in Tristan und Isolde), Zurigo (Penelope nel Ritorno di Ulisse in patria), Salisburgo (Mescalina nel Grand Macabre). Dopo aver interpretato Marie in Wozzeck a Lisbona, nel 2008 completa un progressivo passaggio al repertorio di soprano drammatico debuttando come Elektra al Teatro La Fenice con Inbal. Nel 2009-2010 debutta come Eurydike in Orpheus und Eurydike di Krenek alla Konzerthaus di Berlino con Zagrosek, canta Erwartung di Schoenberg al Capitole di Tolosa e l'Ottava Sinfonia di Mahler a Colonia. Nel 2011 debutta come Brünnhilde in Siegfried e Götterdämmerung all'Opéra Bastille di Parigi con Jordan, canta Rusalka (la principessa straniera) al New National Theatre di Tokyo e Das Lied von der Erde al Brucknerfest di Linz. Nel 2012 è di nuovo Klementia in Sancta Susanna di Hindemith a Ravenna con Muti. Ha collaborato con direttori quali Muti, Jordan, Maazel, Abbado, Harnoncourt, Inbal, Salonen, Welser-Möst, Fedoseyev, e registi quali Sellars, Flimm, Guth, Grüber, Braunschweig, Wilson, Curran. Debutta alla Fenice il ruolo di Isolde.

\section{RichaRd PAUl FinK}

Baritono, interprete del ruolo di Kurwenal. Iniziata la carriera professionale presso la Houston Grand Opera, debutta nel 1998 al Metropolitan di New York e nel 2000 alla Staatsoper di Berlino come Telramund in Lohengrin e nel 2001 all'Opéra di Parigi come Klingsor in Parsifal. Si è esibito nei principali teatri americani (Metropolitan, San Francisco, Houston, Washington, San Diego, Seattle, Los Angeles, Chicago, Santa Fe, Ravinia, Dallas, Philadelphia, Atlanta, Miami, New Orleans, Portland, Tulsa, St. Louis, Boston, Vancouver, Toronto, Montreal, Mexico City) e internazionali (Staatsoper e Deutsche Oper di Berlino, Amburgo, Lipsia, Salisburgo, Bregenz, Parigi, Tolosa, Amsterdam, Cardiff, Edimburgo, Madrid, Venezia, Bologna, Trieste, Spoleto, Tel Aviv, Sydney, Santiago del Cile), collaborando con direttori quali Levine, Abbado, Eschenbach, Conlon, Gergiev, Runnicles, Gatti. Il suo repertorio comprende lavori di Beethoven (Fidelio), Weber (Der Freischütz), Wagner (Der fliegende Holländer, Lohengrin, Tristan und Isolde, Alberich nel Ring, Parsifal), Strauss (Salome, Elektra), d'Albert (Tiefland), Korngold (Die tote Stadt), Berg (Wozzeck), Musorgskij (Chovanščina), Čajkovskij (Evgenij Onegin), Dvořák (Rusalka), Offenbach (Les contes d'Hoffmann), Bizet (Carmen), Saint-Saëns (Samson et Dalila), Verdi (Nabucco, Rigoletto, Macbeth, Aida, Otello), Puccini (Tosca), Mascagni (Cavalleria rusticana), Leoncavallo (Pagliacci), Ponchielli (La Gioconda), Giordano (Andrea Chénier), Stravinskij (The Rake's Progress, Oedipus Rex), Britten (Noye's Fludde), Adams (Nixon in China). Ha inoltre partecipato alle prime assolute di The Great Gatsby di Harbison al Metropolitan e Dr. Atomic di Adams a San Francisco. Nella stagione 2011-2012 è stato Alberich nel Ring (Das Rheingold, Siegfried e Götterdämmerung) al Metropolitan, Kissinger in Nixon in China a Kansas City e Wozzeck a Santa Fe.

\section{MarCeLlo NARDIS}

Tenore, interprete del ruolo di Melot. Ha iniziato lo studio della musica in tenera età diplomandosi in pianoforte, canto e musica vocale da camera. Contemporaneamente si è laureato sia in lettere classiche che in archeologia cristiana. Pianista, ha debuttato come tenore nel 2003 cantando per il Santo Padre in occasione della Giornata Mondiale della Gioventù di Toronto. Da quel momento ha affiancato all'attività teatrale operistica una intensa attività concertistica che lo ha portato ad esibirsi sui principali palcoscenici internazionali, dal Teatro alla Scala al New National Theatre di Tokyo, dall'Accademia Nazionale di Santa Cecilia al Liceu di Barcellona, dal San Car- 
lo di Napoli al Mozarteum di Salisburgo, collaborando con direttori quali Inbal, Muti, Shambadal, Steinberg o Soudant, con formazioni quali i Wiener Philharmoniker o Les Talens lyriques e con pianisti come Shetler, Deutsch, Drake, Canino o Ballista. Particolarmente dedito al repertorio liederistico, è membro onorario della International Schubert Society di New York ed ha all'attivo più di settanta esecuzioni pubbliche della schubertiana Winterreise, talvolta nella doppia veste, contemporaneamente, di pianista e cantante. Al Teatro La Fenice è stato Šujskij in Boris Godunov e, per l'inaugurazione 2012, Hendrik Gillot in Lou Salomé di Giuseppe Sinopoli.

\section{TUIJA KNIHTILÄ}

Mezzosoprano, interprete del ruolo di Brangäne. Nata in Finlandia, ha studiato all'Accademia Sibelius di Helsinki perfezionandosi poi alla Hochschule der Künste di Berlino e all'Internationales Opernstudio dell'Opernhaus di Zurigo. È ospite regolare dell'Opera Nazionale Finlandese di Helsinki e dal 2004 del Festival di Savonlinna. Il suo repertorio include lavori di Mozart (Cherubino nelle Nozze di Figaro, Dorabella in Così fan tutte, seconda dama nella Zauberflöte), Rossini (Angelina nella Cenerentola, Rosina nel Barbiere di Siviglia), Bizet (Carmen), Čajkovskij (Olga in Evgenij Onegin, Polina nella Dama di picche), Wagner (Waltraute nella Walküre, Wellgunde in Götterdämmerung, una fanciulla-fiore in Parsifal), Strauss (Adelaide in Arabella, il compositore in Ariadne auf Naxos), Sallinen (la donna in Ratsumies). In ambito concertistico ha cantato la Missa solemnis di Beethoven e il Requiem di Verdi con le principali orchestre scandinave ed europee collaborando con direttori quali Segerstam, Salonen e Franck. Nelle ultime stagioni ha cantato Dorabella in Cosi fan tutte, Maddalena in Rigoletto, Carmen in Carmen e Amneris in Aida all'Opera Nazionale Finlandese, il compositore in Ariadne auf Naxos e Venus in Tannbäuser alla Norske Opera di Oslo, Brangäne in Tristan und Isolde al Nationaltheater di Weimar (debutto nel ruolo) e a Oslo, Ortrud in Lohengrin al Festival di Savonlinna (debutto nel ruolo).

\section{MirKo GUADAGNINI}

Tenore, interprete del ruolo del pastore. Ha studiato al Conservatorio di Milano. Il suo repertorio spazia dalla musica barocca a Mozart agli autori del Xx secolo. Ha interpretato i ruoli di Enea (Dido and Aeneas), Renaud (Armide di Gluck), Don Ottavio (Don Giovanni), Tamino (Die Zauberflöte), Almaviva (Il barbiere di Siviglia di Paisiello), Cassio (Otello di Verdi), Fenton (Falstaff), Tom Rakewell (The Rake's Progress), Hortensio (Kiss Me Kate di Cole Porter) in prestigiosi teatri italiani ed esteri (Scala, Firenze, Torino, Trieste, Venezia, Jesi, Châtelet di Parigi, Lione, Montpellier, Monte-Carlo, Ginevra, Lisbona, Tel Aviv), con direttori quali Roberto Abbado, Campanella, Chung, Flor, Mehta, Muti, Pidò, Renzetti, Viotti. In ambito barocco ha cantato L'Orfeo di Monteverdi, L'olimpiade di Pergolesi, Alcina e Rinaldo di Händel, con direttori quali Alessandrini, Gardiner, Christie, Neumann, Dantone, Biondi, De Marchi. Ha partecipato alle prime esecuzioni assolute del Dissoluto assolto di Corghi, Antigone di Fedele (Premio Abbiati 2007), Phaedra di Henze (Premio Abbiati 2008), Patto di sangue di D'Amico, Il killer di parole di Ambrosini (Premio Abbiati 2010). Nella stagione 2011-2012 ha cantato Il ritorno di Ulisse in patria alla Scala con Alessandrini e la regia di Wilson, Vèc Makropulos a Firenze con Mehta e la regia di Friedkin e Il killer di parole a Nancy con Molino e la regia di Micheli.

\section{ARMANDO GABBA}

Baritono, interprete del ruolo del pilota. Nato a Parma, vince nel 1982 il Concorso Voci Verdiane di Busseto e canta in Rigoletto con Sinopoli. Perfezionatosi alla Juilliard School di New York, 
svolge un'intensa attività in Italia (Scala, Opera di Roma, Parma, Torino, Genova, Macerata, Firenze, Venezia, Verona, Trieste, Napoli, Palermo, Reggio Emilia, Salerno) e all'estero (Darmstadt, Zurigo, Amburgo, Bonn, Montpellier, Israele, Cipro, Tokyo), in un vasto ed eclettico repertorio comprendente opere di Gluck, Meyerbeer, Gounod, Offenbach, Lalo, Bizet, Massenet, Ponchielli, Leoncavallo, Mascagni, Giordano, Pizzetti, Strauss, Lehár, Berg, Bernstein, Tutino, con una particolare predilezione per i titoli di Verdi e Puccini. Nel 2012 ha cantato Candide di Bernstein all'Opera di Roma e La bohème (Schaunard), La traviata (Douphol) e Rigoletto (Marullo) alla Fenice di Venezia.

\section{GIAN LUCA PASOLINI}

Tenore, interprete del ruolo del giovane marinaio. Laureatosi nel 2002 presso il Conservatorio di Pesaro con Robleto Merolla, si perfeziona all'Accademia di Busseto con Alain Billard e all'Accademia del Maggio Musicale Fiorentino con Bernadette Manca di Nissa e consegue nel 2007 il master in canto lirico presso il Conservatorio di Firenze. Debutta nel 1999 al Teatro Comunale di Bologna nel Barbiere di Siviglia di Paisiello, e da allora si è esibito in Italia (Scala, Bologna, Firenze, Trieste, Jesi, Ravenna, Pesaro, Bergamo, Torino, Roma) ed all'estero (Staatsoper di Berlino, Marsiglia, Copenaghen, San Pietroburgo, Mosca, New York, Los Angeles, Vancouver, Tokyo, Seoul) in lavori di Monteverdi, Scarlatti, Jommelli, Mozart, Nicolini, Rossini, Bellini (Arturo nei Puritani a Seoul), Donizetti (Percy in Anna Bolena a Tokyo), Verdi, Bottesini, Puccini, Pizzetti (Assassinio nella cattedrale alla Scala), Beethoven, Mendelssohn, Mahler, Orff, Halévy, Musorgskii, Stravinskij, Prokof'ev (Il giocatore alla Scala), Šostakovič (Una Lady Macbeth del distretto di Mcensk alla Scala), Knussen (Where the Wild Things Are al Maggio Musicale Fiorentino), Glass, Vacchi (Teneke alla Scala). Ha collaborato con direttori quali Barenboim, Bychkov, Palumbo, Renzetti, Cremonesi, Mehta, Ono, Roberto Abbado, Lü Jia, Handt, Kuhn, Zagrosek, Zedda. Del 2012 il debutto nel ruolo di Paul Rée in Lou Salomé di Sinopoli a Venezia. 
Fondazione Teatro La Fenice di Venezia
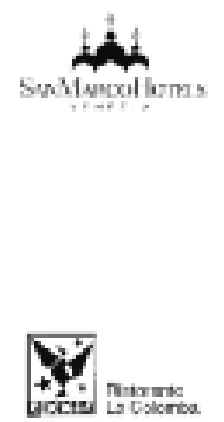

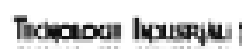

$\overline{z r}$

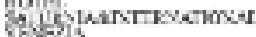

해 항

BAUER

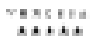

intion

SETE

ABBONATI SOSTENITORI 


\section{Fondazione Teatro La Fenice di Venezia Area Artistica}

\begin{tabular}{lll}
\hline Diego Matheuz & Joyce Fieldsend $\diamond$ & Raffaele Centurioni $\diamond$ \\
direttore principale & maestro di sala & Roberta Ferrari $\diamond$ \\
$\begin{array}{l}\text { Marco Paladin } \diamond \\
\text { direttore dei complessi musicali } \\
\text { dipalcoscenico }\end{array}$ & Luca De Marchi $\diamond$ & maestri di palcoscenico \\
& altro maestro di sala & Roberta Paroletti $\diamond$ \\
& & maestro alle luci
\end{tabular}

\section{Orchestra del Teatro la Fenice}

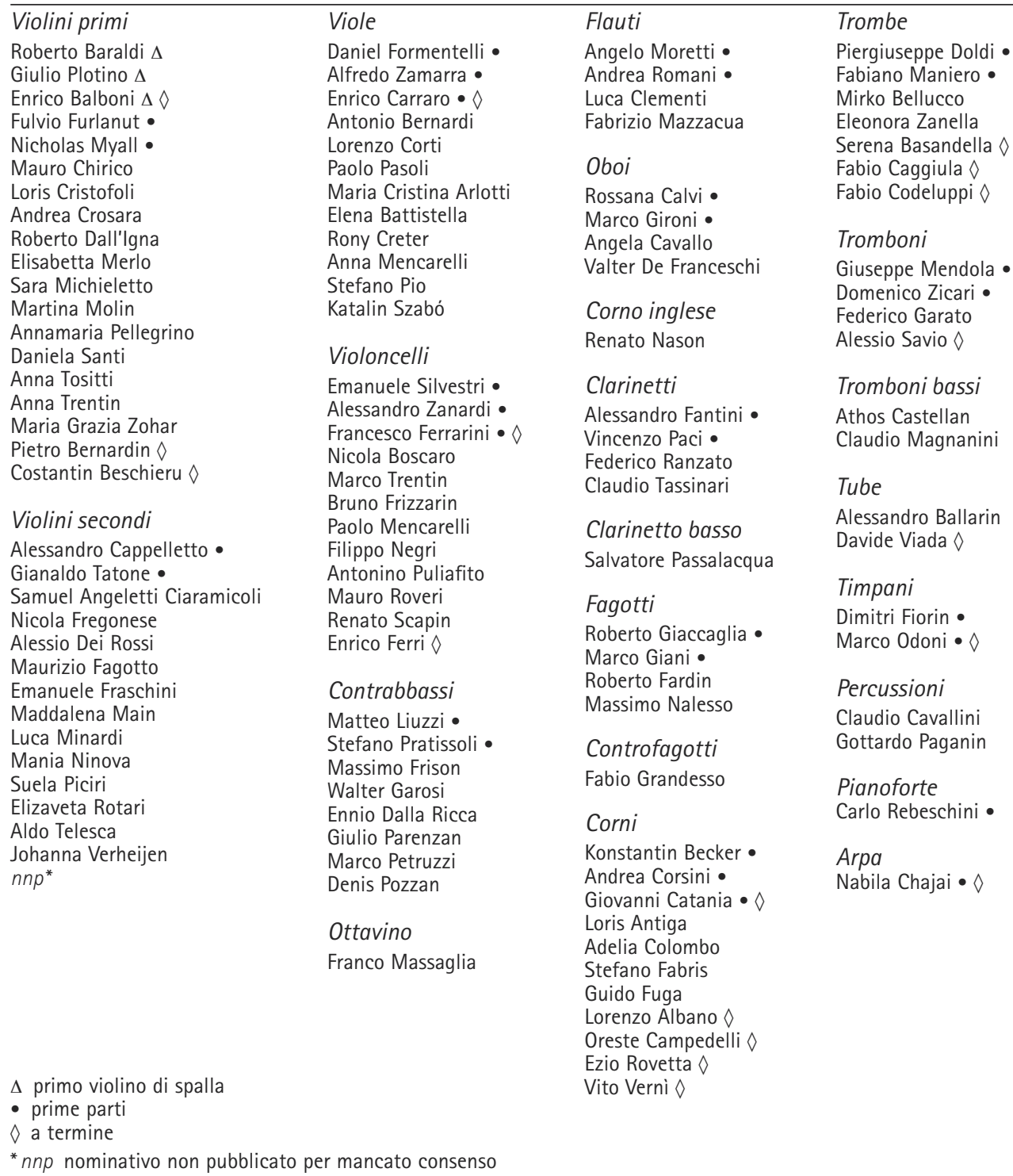




\title{
Fondazione Teatro La Fenice di Venezia Area Artistica
}

\author{
Claudio Marino Moretti Ulisse Trabacchin \\ maestro del Coro altro maestro del Coro
}

\section{Coro del Teatro la Fenice}

\begin{tabular}{llll}
\hline Soprani & Alti & Tenori & Bassi \\
Nicoletta Andeliero & Valeria Arrivo & Domenico Altobelli & Giuseppe Accolla \\
Cristina Baston & Mafalda Castaldo & Ferruccio Basei & Carlo Agostini \\
Lorena Belli & Claudia Clarich & Cosimo D'Adamo & Giampaolo Baldin \\
Anna Maria Braconi & Marta Codognola & Dionigi D'Ostuni & Julio Cesar Bertollo \\
Lucia Braga & Roberta De luliis & $n n p^{*}$ & Antonio Casagrande \\
Mercedes Cerrato & Elisabetta Gianese & Enrico Masiero & Antonio S. Dovigo \\
Emanuela Conti & Lone Kirsten Loëll & Carlo Mattiazzo & Salvatore Giacalone \\
Chiara Dal Bo' & Manuela Marchetto & Stefano Meggiolaro & Umberto Imbrenda \\
Milena Ermacora & Misuzu Ozawa & Roberto Menegazzo & Massimiliano Liva \\
Susanna Grossi & Gabriella Pellos & Dario Meneghetti & Gionata Marton \\
Michiko Hayashi & Francesca Poropat & Ciro Passilongo & Nicola Nalesso \\
Maria Antonietta Lago & Orietta Posocco & Raffaele Pastore & Emanuele Pedrini \\
Loriana Marin & Nausica Rossi & Marco Rumori & Mauro Rui \\
Antonella Meridda & Paola Rossi & Bo Schunnesson & Roberto Spano \\
Alessia Pavan & & Salvatore Scribano & Franco Zanette \\
Lucia Raicevich & Massimo Squizzato & Enzo Borghetti $\diamond$ \\
Andrea Lia Rigotti & & Paolo Ventura & Antonio Corsano $\diamond$ \\
Ester Salaro & Bernardino Zanetti & Francesco De Poli $\diamond$ \\
Elisa Savino & Giuseppe Baglieri $\diamond$ & Emiliano Esposito $\diamond$ \\
& & Giovanni Deriu $\diamond$ & Mirko Quarello $\diamond$ \\
& & Safa Korkmaz $\diamond$ &
\end{tabular}




\section{Fondazione Teatro La Fenice di Venezia Struttura Organizzativa}

\section{SOVRINTENDENZA}

Cristiano Chiarot sovrintendente

Rossana Berti

Cristina Rubini

\section{DIREZIONI OPERATIVE}

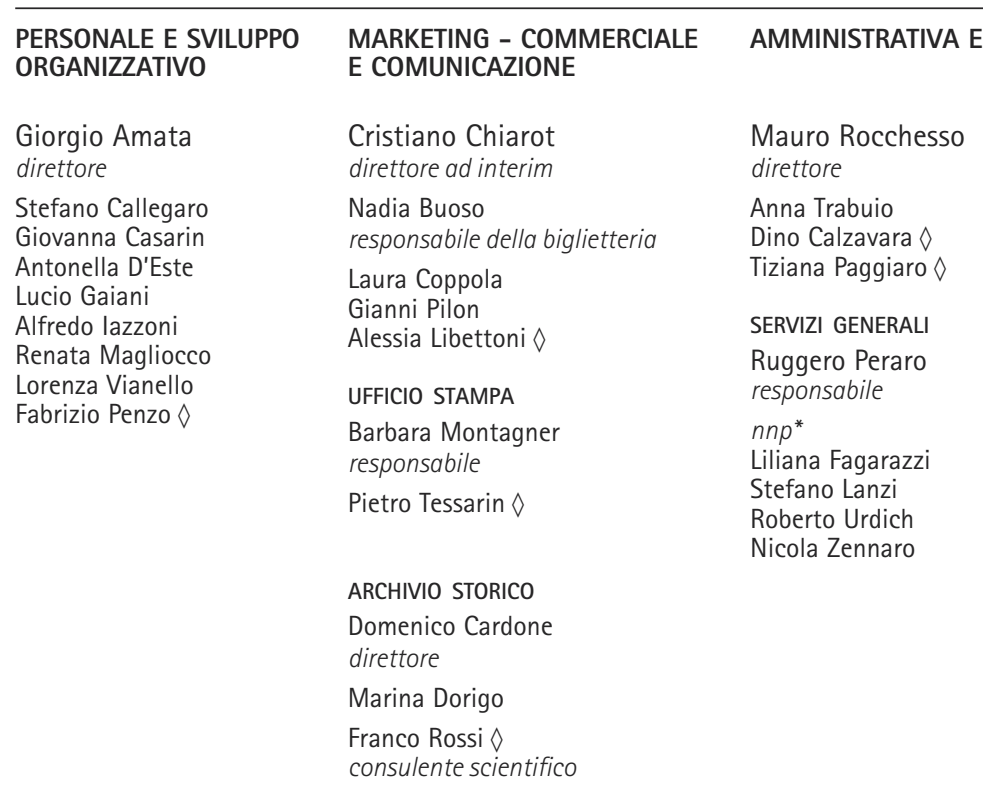

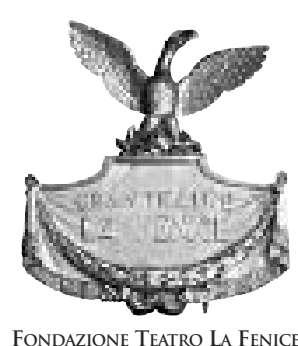
DI VENEZIA 


\section{Fondazione Teatro La Fenice di Venezia Struttura Organizzativa}

\section{DIREZIONE ARTISTICA}

Fortunato Ortombina direttore artistico

Diego Matheuz direttore principale

Bepi Morassi direttore della produzione

Franco Bolletta consulente artistico per la danza

$\begin{array}{ll}\text { SEGRETERIA ARTISTICA } & \begin{array}{l}\text { AREA FORMAZIONE } \\ \text { E MULTIMEDIA }\end{array} \\ \begin{array}{l}\text { Pierangelo Conte } \\ \text { segretario artistico }\end{array} & \begin{array}{l}\text { Simonetta Bonato } \\ \text { responsabile }\end{array} \\ \text { UFFICIO CASTING } & \begin{array}{l}\text { Andrea Giacomini } \\ \text { Thomas Silvestri }\end{array} \\ \text { Anna Migliavacca } & \begin{array}{l}\text { Alessia Pelliciolli } \diamond \\ \text { Monica Fracassetti } \diamond\end{array}\end{array}$

SERVIZI MUSICALI

Cristiano Beda

Salvatore Guarino

Andrea Rampin

Francesca Tondelli

ARCHIVIO MUSICALE

Gianluca Borgonovi

Marco Paladin
DIREZIONE SERVIZI

DI ORGANIZZAZIONE DELLA PRODUZIONE

Lorenzo Zanoni

direttore di scena e

palcoscenico

Valter Marcanzin

Lucia Cecchelin

responsabile produzione

Lucas Christ $\diamond$

Fabio Volpe

Paolo Dalla Venezia
DIREZIONE ALLESTIMENTO

SCENOTECNICO

Massimo Checchetto

direttore

Carmen Attisani

Area tecnica 


\section{Fondazione Teatro La Fenice di Venezia Area Tecnica}

\begin{tabular}{|c|c|c|c|c|c|}
\hline $\begin{array}{l}\text { Macchinisti, } \\
\text { falegnameria, } \\
\text { magazzini }\end{array}$ & Elettricisti & Audiovisivi & Attrezzeria & $\begin{array}{l}\text { Interventi } \\
\text { scenografici }\end{array}$ & $\begin{array}{l}\text { Sartoria } \\
\text { e vestizione }\end{array}$ \\
\hline $\begin{array}{l}\text { Massimiliano Ballarini } \\
\text { capo reparto }\end{array}$ & $\begin{array}{l}\text { Vilmo Furian } \\
\text { capo reparto }\end{array}$ & $\begin{array}{l}\text { Alessandro Ballarin } \\
\text { capo reparto }\end{array}$ & $\begin{array}{l}\text { Roberto Fiori } \\
\text { capo reparto }\end{array}$ & Marcello Valonta & $\begin{array}{l}\text { Carlos Tieppo } \diamond \\
\text { capo reparto }\end{array}$ \\
\hline $\begin{array}{l}\text { Andrea Muzzati } \\
\text { vice capo reparto } \\
\text { Roberto Rizzo } \\
\text { vice capo reparto } \\
\text { Paolo De Marchi } \\
\text { responsabile } \\
\text { falegnameria } \\
\text { Michele Arzenton } \\
\text { Roberto Cordella } \\
\text { Antonio Covatta } \\
\text { nnp* } \\
\text { Dario De Bernardin } \\
\text { Roberto Gallo } \\
\text { Michele Gasparini } \\
\text { Roberto Mazzon } \\
\text { Carlo Melchiori } \\
\text { Francesco Nascimben } \\
\text { Francesco Padovan } \\
\text { Claudio Rosan } \\
\text { Stefano Rosan } \\
\text { Paolo Rosso }\end{array}$ & $\begin{array}{l}\text { Fabio Barettin } \\
\text { vice capo reparto } \\
\text { Costantino Pederoda } \\
\text { vice capo reparto } \\
\text { Alberto Bellemo } \\
\text { Andrea Benetello } \\
\text { Marco Covelli } \\
\text { Federico Geatti } \\
\text { Roberto Nardo } \\
\text { Maurizio Nava } \\
\text { Marino Perini } \\
\text { nnp* } \\
\text { Alberto Petrovich } \\
\text { nnp* } \\
\text { Teodoro Valle } \\
\text { Giancarlo Vianello } \\
\text { Massimo Vianello } \\
\text { Roberto Vianello } \\
\text { Alessandro Diomede } \diamond \\
\text { Luca Seno } \downarrow \\
\text { Michele Voltan } \diamond\end{array}$ & $\begin{array}{l}\text { Michele Benetello } \\
\text { Cristiano Faè } \\
\text { Stefano Faggian } \\
\text { Tullio Tombolani } \\
\text { Marco Zen } \\
\text { Giuseppe Bottega } \downarrow\end{array}$ & $\begin{array}{l}\text { Sara Valentina } \\
\text { Bresciani } \\
\text { vice capo reparto } \\
\text { Salvatore De Vero } \\
\text { Vittorio Garbin } \\
\text { Romeo Gava } \\
\text { Dario Piovan } \\
\text { Paola Ganeo } \diamond \\
\text { Roberto Pirrò } \diamond\end{array}$ & & $\begin{array}{l}\text { Tebe Amici } \\
\text { Bernadette Baudhuin } \\
\text { Emma Bevilacqua } \\
\text { Luigina Monaldini } \\
\text { Valeria Boscolo } \diamond \\
\text { Stefania Mercanzin } \diamond \\
\text { Paola Milani } \\
\text { addetta calzoleria }\end{array}$ \\
\hline
\end{tabular}

Massimo Senis

Luciano Tegon

Mario Visentin

Andrea Zane

Vitaliano Bonicelli $\diamond$

Pierluca Conchetto $\diamond$

Franco Contini $\diamond$

Cristiano Gasparini $\diamond$

Enzo Martinelli $\diamond$

Stefano Neri $\diamond$

Giovanni Pancino $\diamond$

Paolo Scarabel $\diamond$

\footnotetext{
$\diamond$ a termine

${ }^{*}$ nnp nominativo non pubblicato per mancato consenso
}

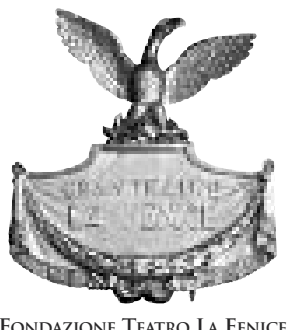

Fondazione Teatro La Fenice DI VENEZIA 
LIRICA E BALLETTO 2012-2013



\section{scene e costumi Robert Innes \\ Hopkins}

Orchestra e Coro

del Teatro La Fenice

maestro del Coro

Claudio Marino Moretti

nuovo allestimento Fondazione Teatro La Fenice nel bicentenario della nascita di

Richard Wagner

spettacolo sostenuto dal Freundeskreis des Teatro La Fenice

Teatro La Fenice

18 / 20 / 22 / 24 / 26 gennaio 2013

\section{masnadieri}

personaggi interpreti principali

Carlo Andeka Gorrotxategui

Francesco Artur Ruciński

Amalia Maria Agresta

Daniele Rustioni

regia Gabriele Lavia

scene Alessandro Camera

Orchestra e Coro

maestro del Coro

Claudio Marino Moretti

an Carlo di Napoli nel bicentenario della

Claudio Marino Morett

Fondazione Teatro La Fenice

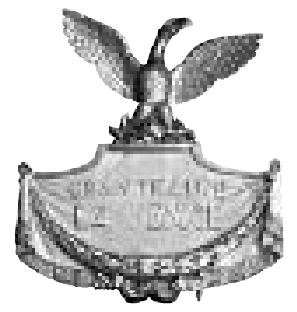

Fondazione Teatro La Fenice DI VENEZIA 
Teatro La Fenice

15 / 17 / 19 / 21 / 23 marzo 2013

Věc Makropulos

(L'affare Makropulos)

musica di Leoš Janáček

prima rappresentazione a Venezia

personaggi interpreti principali

Emilia Marty Ángeles Blancas Gulín

Albert Gregor Ladislav Elgr

Jaroslav Prus Martin Bárta

maestro concertatore e direttore

Gabriele Ferro

regia Robert Carsen

scene Radu Boruzescu

costumi Miruna Boruzescu

Orchestra e Coro

del Teatro La Fenice

maestro del Coro

Claudio Marino Moretti

nuovo allestimento Fondazione Teatro La Fenice in coproduzione con Opéra National du Rhin di Strasburgo e Staatstheater di Norimberga

\section{Teatro Malibran}

16 / 20 / 22 / 24 / 28 marzo

12 / 14 / 16 / 17 aprile 2013

\section{La cambiale di matrimonio} musica di Gioachino Rossini personaggi e interpreti principali Tobia Mill Omar Montanari Fanni Marina Bucciarelli Edoardo Milfort Giorgio Misseri Slook Marco Filippo Romano

maestro concertatore e direttore

Stefano Montanari

regia Enzo Dara

scene e costumi

Scuola di Scenografia dell'Accademia di Belle Arti di Venezia

Orchestra del Teatro La Fenice Orchestra del Conservatorio Benedetto Marcello di Venezia (12 / 14 / 16 / 17 aprile)

nuovo allestimento

Fondazione Teatro La Fenice

nell'ambito del progetto

Atelier della Fenice al Teatro Malibran
Teatro La Fenice

30 aprile - 28 maggio 2013

Progetto Mozart

imaestro concertatore e direttore

Antonello Manacorda

regia Damiano Michieletto

scene Paolo Fantin

costumi Carla Teti

Orchestra e Coro

del Teatro La Fenice

maestro del Coro

Claudio Marino Moretti

30 aprile $4 / 10 / 14 / 17 / 21 / 24 / 28$

maggio 2013

\section{Don Giovanni}

musica di

Wolfgang Amadeus Mozart

personaggi interpreti principali

Don Giovanni Simone Alberghini /

Alessio Arduini / Markus Werba

Donna Anna Carmela Remigio / Maria Bengtsson

Don Ottavio Marlin Miller

Donna Elvira Maria Pia Piscitelli

Leporello Nicola Ulivieri

allestimento Fondazione Teatro La Fenice

5 / 11 / 15 / 18 / 22 / 25 maggio 2013

Le nozze di Figaro

musica di

Wolfgang Amadeus Mozart

personaggi e interpreti principali

I/ conte di Almaviva Simone Alberghini

La contessa di Almaviva Marita Solberg

Susanna Rosa Feola

Figaro Vito Priante

allestimento Fondazione Teatro La Fenice

12 / 16 / 19 / 23 / 26 maggio 2013

\section{Cosi fan tutte}

musica di

Wolfgang Amadeus Mozart

personaggi e interpreti principali

Fiordiligi Maria Bengtsson

Dorabella Josè Maria Lo Monaco

Guglielmo Alessio Arduini

Despina Caterina Di Tonno

Don Alfonso Luca Tittoto

allestimento Fondazione Teatro La Fenice
Teatro La Fenice

21 / 22 / 23 / 25 / 26 / 27 / 28 / 29 / 30

giugno 2013

\section{Madama Butterfly}

musica di Giacomo Puccini

personaggi e interpreti principali

Cio-Cio-San Amarilli Nizza / Svetlana Kasyan

F. B. Pinkerton Andeka Gorrotxategui / Giuseppe Varano

Sharpless Vladimir Stoyanov / Elia

Fabbian

maestro concertatore e direttore

Omer Meir Wellber

scene e costumi Mariko Mori

Orchestra e Coro

del Teatro La Fenice

maestro del Coro

Claudio Marino Moretti

nuovo allestimento Fondazione Teatro La

Fenice in collaborazione con la Biennale di

Venezia, evento speciale della 55.

Esposizione Internazionale d'Arte

spettacolo sostenuto dal Circolo La Fenice

Cortile di Palazzo Ducale

10 / 14 / 17 luglio 2013

\section{Otello}

musica di Giuseppe Verdi

maestro concertatore e direttore

Myung-Whun Chung

regia

Francesco Micheli

scene Edoardo Sanchi

costumi Silvia Aymonino

Orchestra e Coro

del Teatro La Fenice

maestro del Coro

Claudio Marino Moretti

nuovo allestimento Fondazione Teatro

La Fenice nel bicentenario della nascita

di Giuseppe Verdi nell'ambito del festival

"Lo spirito della musica di Venezia" 
LiRICA E BALLETTO 2012-2013

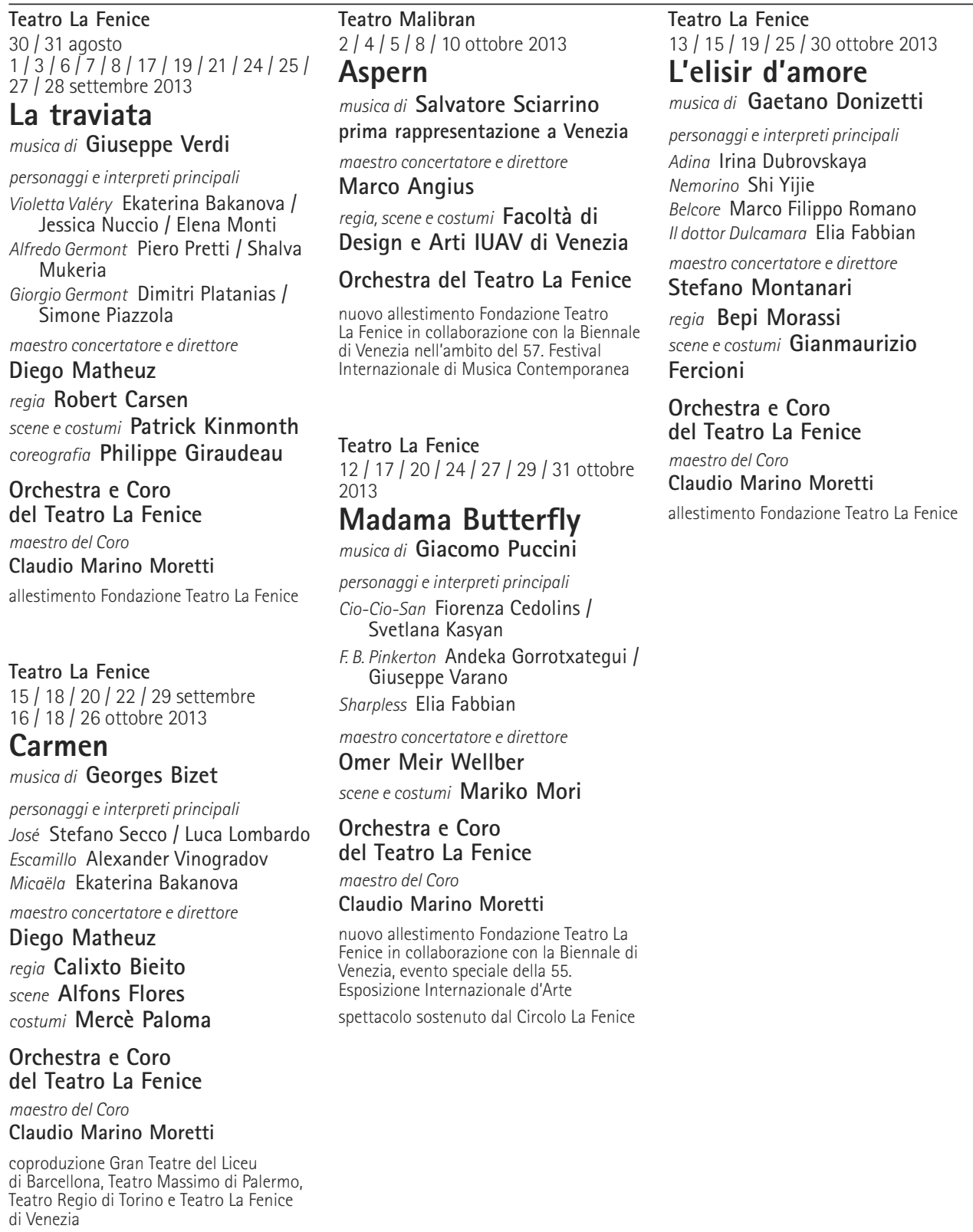


LIRICA E BALLETTO 2013-2014

Teatro La Fenice
22 / 24 / 26 / 28 / 30 novembre
3 dicembre 2013
L'africaine
(L'africana)
musica di Giacomo Meyerbeer
personaggi e interpreti principali
Inès Jessica Pratt
Vasco de Gama Gregory Kunde
Sélika Veronica Simeoni
maestro concertatore e direttore
Emmanuel Villaume
regia Leo Muscato
scene Massimo Checchetto
costumi Carlos Tieppo
Orchestra e Coro
del Teatro La Fenice
maestro del Coro
Claudio Marino Moretti

nuovo allestimento Fondazione Teatro

La Fenice nel $150^{\circ}$ anniversario della morte di Giacomo Meyerbeer

\section{Teatro La Fenice}

18 / 19 / 20 / 21 / 22 dicembre 2013

Eifman Ballet di San Pietroburgo

\section{Onegin}

prima rappresentazione italiana

coreografia di Boris Eifman

musiche di Pëtr II'č Čajkovskij e

Aleksandr Sitkovetskij

interpreti

primi ballerini, solisti e corpo di ballo

dell'Eifman Ballet di San Pietroburgo

scene Zinovy Margolin

costumi Olga Šaišmelašvili, Pëtr

Okunev
Teatro Malibran

17 / 19 / 21 / 23 / 25 gennaio 2014

La scala di seta musica di Gioachino Rossini

regia Bepi Morassi

scene, costumi eluci Scuola di

scenografia dell'Accademia di

Belle Arti di Venezia

Orchestra del Teatro La Fenice

nuovo allestimento Fondazione Teatro La Fenice nell'ambito del progetto Atelier della Fenice al Teatro Malibran

Teatro La Fenice

24 / 26 / 28 / 30 gennaio

1 febbraio 2014

\section{La clemenza di Tito} musica di Wolfgang Amadeus Mozart

maestro concertatore e direttore

Ottavio Dantone

regia Ursel e Karl-Ernst Herrmann

scene e costumi Karl-Ernst

Herrmann

Orchestra e Coro

del Teatro La Fenice

maestro del Coro

Claudio Marino Moretti

allestimento Teatro Real di Madrid

Teatro La Fenice

15 / 16 / 19 / 21 / 23 / 25 / 27 febbraio

4 / 6 / 8 marzo 2014

La traviata

musica di Giuseppe Verdi

maestro concertatore e direttore

Diego Matheuz

regia Robert Carsen

scene e costumi Patrick Kinmonth

coreografia Philippe Giraudeau

Orchestra e Coro

del Teatro La Fenice

maestro del Coro

Claudio Marino Moretti

allestimento Fondazione Teatro La Fenice
Teatro La Fenice

20 / 22 / 26 febbraio

2 / 5 / 7 / 9 marzo 2014

Il barbiere di Siviglia musica di Gioachino Rossini

personaggi e interpreti principali

Il conte d'Almaviva Giorgio Misseri

Figaro Julian Kim

maestro concertatore e direttore

Diego Matheuz

regia Bepi Morassi

scene e costumi Lauro Crisman

Orchestra e Coro

del Teatro La Fenice

maestro de/ Coro

Claudio Marino Moretti

allestimento Fondazione Teatro La Fenice

Teatro Malibran

28 febbraio

1 / 5 / 7 / 11 marzo 2014

\section{II campiello} musica di Ermanno Wolf-Ferrari

maestro concertatore e direttore

\section{Stefano Romani}

regia Paolo Trevisi

Orchestra Regionale Filarmonia

Veneta

Coro Lirico Veneto

allestimento Teatro Sociale di Rovigo progetto "I teatri del Veneto alla Fenice" 
Teatro Malibran

27 / 29 marzo

2 / 4 / 6 aprile 2014

\section{Elegy for Young Lovers}

(Elegia per giovani amanti) musica di Hans Werner Henze regia, scene e costumi Pier Luigi Pizzi

Orchestra del Teatro La Fenice

allestimento Fondazione Teatro delle Muse di Ancona

Teatro La Fenice

28 / 30 marzo

$1 / 3$ / 5 aprile 2014

II trionfo del tempo e del disinganno musica di Georg Friedrich Händel regia Calixto Bieito scene Susanne Gschwender costumi Anna Eiermann

Orchestra del Teatro La Fenice allestimento Staatstheater Stuttgart
Teatro La Fenice

19 aprile - 1 giugno 2013

Progetto Puccini

Orchestra e Coro

del Teatro La Fenice

maestro del Coro

Claudio Marino Moretti

19 / 22 / 24 / 27 / 29 aprile

3 / 10 / 25 / 27 / 30 maggio 2014

\section{La bohème}

musica di Giacomo Puccini

regia Francesco Micheli

scene Edoardo Sanchi

costumi Silvia Aymonino

allestimento Fondazione Teatro La Fenice

26 / 30 aprile

2 / 4 / 9 / 21 / 24 / 29 maggio

1 giugno 2014

Madama Butterfly

musica di Giacomo Puccini

maestro concertatore e direttore

Omer Meir Wellber

scene e costumi Mariko Mori

allestimento Fondazione Teatro La Fenice

16 / 17 / 18 / 20 / 22 / 23 / 28 / 31 maggio 2014

\section{Tosca}

musica di Giacomo Puccini

personaggi e interpreti principali

Mario Cavaradossi Francesco Meli

maestro concertatore e direttore

Daniele Callegari

regia Serena Sinigaglia

nuovo allestimento Fondazione Teatro

La Fenice
Teatro La Fenice

27 / 29 giugno

1 / 3 / 5 luglio 2014

\section{The Rake's Progress}

(La carriera di un libertino)

musica di Igor Stravinskij

personaggi e interpreti principali

Nick Shadow Alex Esposito

maestro concertatore e direttore

Diego Matheuz

regia Damiano Michieletto

scene Paolo Fantin

costumi Carla Teti

Orchestra e Coro

del Teatro La Fenice

maestro del Coro

Claudio Marino Moretti

nuovo allestimento Fondazione Teatro

La Fenice in coproduzione con Oper Leipzig

nell'ambito del festival "Lo spirito della

musica di Venezian

Cortile di Palazzo Ducale

12 / 15 / 18 luglio 2014

\section{Otello}

musica di Giuseppe Verdi

regia Francesco Micheli

scene Edoardo Sanchi

costumi Silvia Aymonino

Orchestra e Coro

del Teatro La Fenice

maestro del Coro

Claudio Marino Moretti

allestimento Fondazione Teatro La Fenice nell'ambito del festival "Lo spirito della

musica di Venezian 
LIRICA E BALLETTO 2013-2014

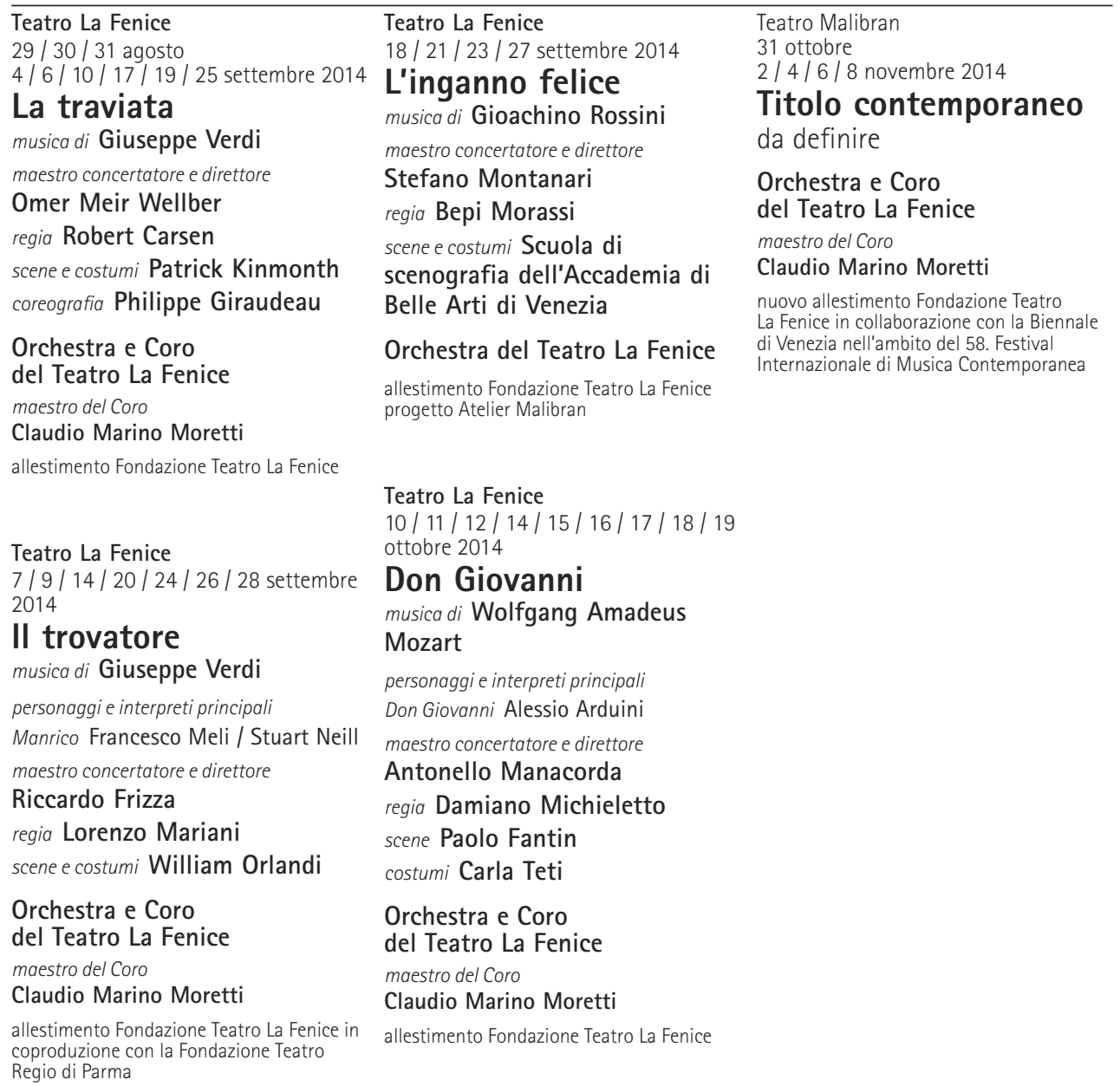

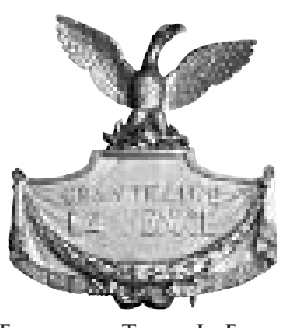

Fondazione Teatro La Fenice DI VenEZia 
STAGIONE SINFONICA 2012-2013

Teatro La Fenice

5 ottobre 2012 ore 20.00 turno S

7 ottobre 2012 ore 17.00 turno U

direttore

\section{Diego Matheuz}

\section{Maurice Ravel}

Pavane pour une infante défunte per piccola orchestra

Wolfgang Amadeus Mozart

Concerto per pianoforte e orchestra in re minore KV 466

pianoforte Leonardo Pierdomenico

vincitore del Premio Venezia 2011

Pëtr II'ič Čajkovskij

Sinfonia n. 6 in si minore op. 74

Patetica

Orchestra del Teatro La Fenice

Teatro La Fenice

22 ottobre 2012 ore 20.00 turno S

direttore

\section{Yuri Temirkanov}

Pëtr Il'ič Čajkovskij

Suite dal balletto Lo schiaccianoci

\section{Modest Musorgskij}

Quadri di un'esposizione

trascrizione per orchestra di Maurice Ravel

Orchestra dell'Accademia

Teatro alla Scala

Teatro La Fenice

7 dicembre 2012 ore 20.00 turno S

9 dicembre 2012 ore 17.00 turno U

direttore

\section{Diego Matheuz}

Pëtr II'ič Čajkovskij

Sinfonia n. 2 in do minore op. 17

Piccola Russia

Sinfonia $n .1$ in sol minore op. 13

Sognid'inverno

Orchestra del Teatro La Fenice
Basilica di San Marco

13 dicembre 2012 ore 20.00 solo per invito

14 dicembre 2012 ore 20.00 turno S

direttore

\section{Stefano Montanari}

musiche dal repertorio marciano

Orchestra del Teatro La Fenice

in collaborazione con la Procuratoria di San Marco

Teatro La Fenice

22 febbraio 2013 ore 20.00 turno S

23 febbraio 2013 ore 17.00 turno U

direttore

\section{Diego Matheuz}

Wolfgang Amadeus Mozart

Sinfonia n. 29 in la maggiore KV 201

Sinfonia concertante per fiati

e orchestra in mi bemolle maggiore

KV Anh. I, 9

Pëtr II'ič Čajkovskij

Sinfonia n. 5 in mi minore op. 64

Orchestra del Teatro La Fenice

Teatro Malibran

1 marzo 2013 ore 20.00 turno S

2 marzo 2013 ore 17.00 turno U

direttore

\section{Diego Matheuz}

Gianluca Cascioli

Trasfigurazione

composizione vincitrice del I Concorso

Francesco Agnello

Pëtr II'ič Čajkovskij

Variazioni su un tema rococò per violoncello e orchestra in la maggiore op. 33

Sinfonia n. 3 in re maggiore op. 29

Polacca

Orchestra del Teatro La Fenice
Teatro La Fenice

22 marzo 2013 ore 20.00 turno S 24 marzo 2013 ore 17.00 turno U

direttore

\section{Gabriele Ferro}

\section{Edoardo Micheli}

Nuova commissione nell'ambito

del progetto Nuova musica alla Fenice

Igor Stravinskij

Pulcinella, suite per orchestra da camera

\section{Sergej Prokof'ev}

Sinfonia n. 1 in re maggiore op. 25 Classica

Orchestra del Teatro La Fenice

\section{Teatro Malibran}

26 aprile 2013 ore 20.00 turno S 28 aprile 2013 ore 17.00 turno U direttore

\section{Claudio Scimone}

Wolfgang Amadeus Mozart

Sinfonia n. 38 in re maggiore KV 504 Praga

Concerto per fagotto e orchestra in si bemolle maggiore KV 191

Sinfonia n. 35 in re maggiore KV 385 Haffner

Orchestra del Teatro La Fenice

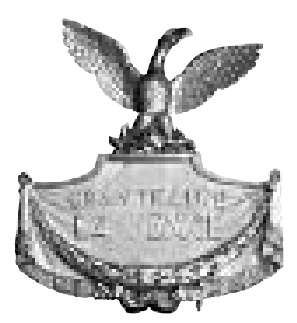

Fondazione Teatro La Fenice DI VENEZIA 
STAGIONE SINFONICA 2012-2013

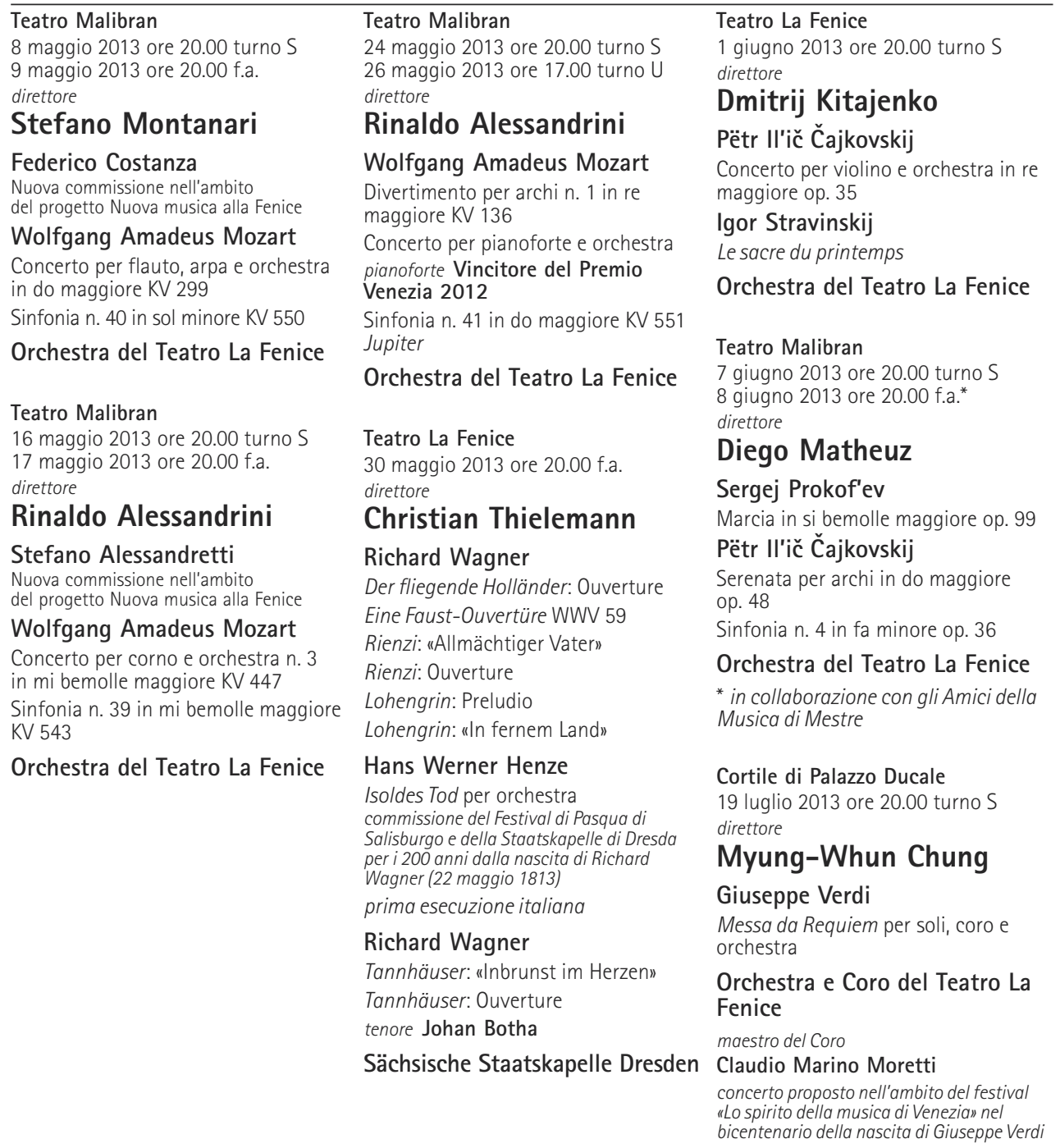




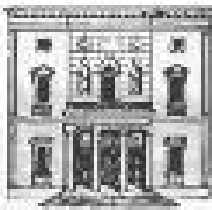 \\ Fond ZZOONE AMICI DELLA FeNICE}

Il Teatro La Fenice, nato nel 1792 dalle ceneri del vecchio Teatro San Benedetto per opera di Giannantonio Selva, appartiene al patrimonio culturale di Venezia e del mondo intero: come ha confermato l'ondata di universale commozione dopo l'incendio del gennaio 1996 e la spinta di affettuosa partecipazione che ha accompagnato la rinascita a nuova vita della Fenice, ancora una volta risorta dalle sue ceneri.

Imprese di questo impegno spirituale e materiale, nel quadro di una società moderna, hanno bisogno di essere appoggiate e incoraggiate dall'azione e dall'iniziativa di istituzioni e persone private: in tale prospettiva si è costituita nel 1979 l'Associazione "Amici della Fenice», con lo scopo di sostenere e affiancare il Teatro nelle sue molteplici attività e d'incrementare l'interesse attorno ai suoi allestimenti e ai suoi programmi. La Fondazione Amici della Fenice attende la risposta degli appassionati di musica e di chiunque abbia a cuore la storia teatrale e culturale di Venezia: da Voi, dalla Vostra partecipazione attiva, dipenderà in misura decisiva il successo del nostro progetto. Sentitevi parte viva del nostro Teatro! Associatevi dunque e fate conoscere le nostre iniziative a tutti gli amici della musica, dell'arte e della cultura.

\section{Quote associative}

Ordinario $€ 60$ Benemerito $€ 250$

Sostenitore $€ 120$ Donatore $€ 500$

I versamenti vanno effettuati su

Iban: IT50Q0634502000100000007406

Cassa di Risparmio di Venezia,

Gruppo Intesa San Paolo

intestati a

Fondazione Amici della Fenice

Campo San Fantin 1897, San Marco

30124 Venezia

Tel e fax: 0415227737

\section{Consiglio direttivo}

Luciana Bellasich Malgara, Alfredo Bianchini, Carla Bonsembiante, Jaja Coin Masutti, Emilio Melli, Antonio Pagnan, Orsola Spinola, Paolo Trentinaglia de Daverio, Barbara di Valmarana

Presidente Barbara di Valmarana

Tesoriere Luciana Bellasich Malgara

Revisori dei conti Carlo Baroncini, Gianguido Ca' Zorzi

Contabilità Nicoletta di Colloredo

Segreteria organizzativa Maria Donata Grimani Viaggi musicali Teresa De Bello

I soci hanno diritto a:

- Inviti a conferenze di presentazione delle opere in cartellone

- Partecipazione a viaggi musicali organizzati per i soci

- Inviti ad iniziative e manifestazioni musicali

- Inviti al «Premio Venezia», concorso pianistico

- Sconti al Fenice-bookshop

- Visite guidate al Teatro La Fenice

- Prelazione nell'acquisto di abbonamenti e biglietti fino ad esaurimento dei posti disponibili

- Invito alle prove aperte per i concerti e le opere

Le principali iniziative della Fondazione

- Restauro del Sipario Storico del Teatro La Fenice: olio su tela di $140 \mathrm{mq}$ dipinto da Ermolao Paoletti nel 1878, restauro eseguito grazie al contributo di Save Venice Inc.

- Commissione di un'opera musicale a Marco Di Bari nell'occasione dei 200 anni del Teatro La Fenice

- Premio Venezia Concorso Pianistico

- Incontri con l'opera 


\section{INIZIATIVE PER IL TEATRO DOPO L'INCENDIO EFFETTUATE GRAZIE AL CONTO «RICOSTRUZIONE»}

\section{Restauri}

- Modellino ligneo settecentesco del Teatro La Fenice dell'architetto Giannantonio Selva, scala 1: 25

- Consolidamento di uno stucco delle Sale Apollinee

- Restauro del sipario del Teatro Malibran con un contributo di Yoko Nagae Ceschina

\section{Donazioni}

Sipario del Gran Teatro La Fenice offerto da Laura Biagiotti a ricordo del marito Gianni Cigna

\section{Acquisti}

- Due pianoforti a gran coda da concerto Steinway

- Due pianoforti da concerto Fazioli

- Due pianoforti verticali Steinway

- Un clavicembalo

- Un contrabbasso a 5 corde

- Un Glockenspiel

- Tube wagneriane

- Stazione multimediale per Ufficio Decentramento

\section{PUBBLICAZIONI}

Il Teatro La Fenice. I progetti, l'architettura, le decorazioni, di Manlio Brusatin e Giuseppe Pavanello, con un saggio di Cesare De Michelis, Venezia, Albrizzi, 1987¹ $1996^{2}$ (dopo l'incendio);

Il Teatro La Fenice. Cronologia degli spettacoli, 1792-1991, di Michele Girardi e Franco Rossi, con il contributo di Yoko Nagae Ceschina, 2 volumi, Venezia, Albrizzi, 1989-1992;

Gran Teatro La Fenice, a cura di Terisio Pignatti, con note storiche di Paolo Cossato, Elisabetta Martinelli Pedrocco, Filippo Pedrocco, Venezia, Marsilio, 1981 $1984^{2}$, 1994³

L'immagine e la scena. Bozzetti e figurini dall'archivio del Teatro La Fenice, 1938-1992, a cura di Maria Ida Biggi, Venezia, Marsilio, 1992;

Giuseppe Borsato scenografo alla Fenice, 1809-1823, a cura di Maria Ida Biggi, Venezia, Marsilio, 1995 ;

Francesco Bagnara scenografo alla Fenice, 1820-1839, a cura di Maria Ida Biggi, Venezia, Marsilio, 1996;

Giuseppe e Pietro Bertoja scenografi alla Fenice, 1840-1902, a cura di Maria Ida Biggi e Maria Teresa Muraro, Venezia, Marsilio, 1998;

Il concorso per la Fenice 1789-1790, di Maria Ida Biggi, Venezia, Marsilio, 1997;

I progetti per la ricostruzione del Teatro La Fenice, 1997, Venezia, Marsilio, 2000;

Teatro Malibran, a cura di Maria Ida Biggi e Giorgio Mangini, con saggi di Giovanni Morelli e Cesare De Michelis, Venezia, Marsilio, 2001;

La Fenice 1792-1996. Il teatro, la musica, il pubblico, l'impresa, di Anna Laura Bellina e Michele Girardi, Venezia, Marsilio, 2003;

Il mito della fenice in Oriente e in Occidente, a cura di Francesco Zambon e Alessandro Grossato, Venezia, Marsilio, 2004;

Pier Luigi Pizzi alla Fenice, a cura di Maria Ida Biggi, Venezia, Marsilio, 2005;

A Pier Luigi Pizzi. 80, a cura di Maria Ida Biggi, Venezia, Amici della Fenice, 2010.

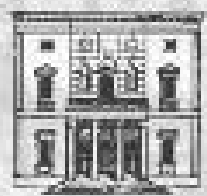




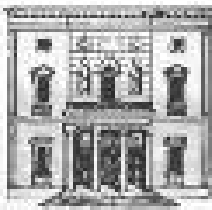 \\ Fond ZZOONE Amici Della Fenice}

Built in 1792 by Gian Antonio Selva, Teatro La Fenice is part of the cultural heritage of not only Venice but also the whole world, as was shown so clearly by the universal emotion expressed after the fire in January 1996 and the moving participation that was behind the rebirth of La Fenice, which once again arose from the ashes.

In modern-day society, enterprises of spiritual and material commitment such as these need the support and encouragement of actions and initiatives by private institutions and figures. Hence, in 1979, the Association "Amici della Fenice" was founded with the aim of supporting and backing the Opera House in its multiple activities and increasing interest in its productions and programmes.

The new Fondazione Amici della Fenice [Friends of La Fenice Foundation] is awaiting an answer from music lovers or anyone who has the opera and cultural history of Venice at heart: the success of our project depends considerably on you, and your active participation.

Make yourself a living part of our Theatre!

Become a member and tell all your friends of music, art and culture about our initiatives.

Membership fee

Regular Friend $€ 60$

Supporting Friend $€ 120$

Honoray Friend $€ 250$

Premium Friend $€ 500$

To make a payment:

Iban: IT50Q0634502000100000007406

Cassa di Risparmio di Venezia,

Gruppo Intesa San Paolo

In the name of

Fondazione Amici della Fenice

Campo San Fantin 1897, San Marco

30124 Venezia

Tel and fax: +390415227737

\section{Board of Directors}

Luciana Bellasich Malgara, Alfredo Bianchini, Carla Bonsembiante, Jaja Coin Masutti, Emilio Melli, Antonio Pagnan, Orsola Spinola, Paolo Trentinaglia de Daverio, Barbara di Valmarana

President Barbara di Valmarana

Treasurer Luciana Bellasich Malgara

Auditors Carlo Baroncini, Gianguido Ca' Zorzi Accounting Nicoletta di Colloredo

Organizational secretary Maria Donata Grimani

Music trips Teresa De Bello

Members have the right to:

- Invitations to conferences presenting performances in the season's programme

- Take part in music trips organized for the members

- Invitations to music initiatives and events

- Invitations to "Premio Venezia», piano competition

- Discounts at the Fenice-bookshop

- Guided tours of Teatro La Fenice

- First refusal in the purchase of season tickets and tickets as long as seats are available

- Invitation to rehearsals of concerts and operas open to the public

\section{The main initiatives of the Foundation}

- Restoration of the historic curtain of Teatro La Fenice: oil on canvas, $140 \mathrm{~m} 2$ painted by Ermolao Paoletti in 1878, restoration made possible thanks to the contribution by Save Venice Inc.

- Commissioned Marco Di Bari with an opera to mark the $200^{\text {th }}$ anniversary of Teatro La Fenice

- Premio Venezia Piano Competition

- Meetings with opera 


\section{THE TEATRO'S INITIATIVES AFTER THE FIRE MADE POSSIBLE THANKS TO THE «RECONSTRUCTION» BANK ACCOUNT}

\section{Restorations}

- Eighteenth-century wooden model of Teatro La Fenice by the architect Giannantonio Selva, scale $1: 25$

- Restoration of one of the stuccos in the Sale Apollinee

- Restoration of the curtain in Teatro Malibran with a contribution from Yoko Nagae Ceschina

Donations

Curtain of Gran Teatro La Fenice donated by Laura Biagiotti in memory of her husband Gianni Cigna

\section{Purchases}

- Two Steinway concert grand pianos

- Two Fazioli concert pianos

- Two upright Steinway pianos

- One harpsichord

- A 5-string double bass

- A Glockenspiel

- Wagnerian tubas

- Multi-media station for Decentralised Office

\section{PUBLICATIONS}

Il Teatro La Fenice. I progetti, l'architettura, le decorazioni, by Manlio Brusatin and Giuseppe Pavanello, with the essay of Cesare De Michelis, Venezia, Albrizzi, 19871, $1996^{2}$ (after the fire);

Il Teatro La Fenice. Cronologia degli spettacoli, 1792-1991, by Franco Rossi and Michele Girardi, with the contribution of Yoko Nagae Ceschina, 2 volumes, Venezia, Albrizzi, 1989-1992;

Gran Teatro La Fenice, ed. by Terisio Pignatti, with historical notes of Paolo Cossato, Elisabetta Martinelli Pedrocco, Filippo Pedrocco, Venezia, Marsilio, 1981 I, 1984 II, 1994 III;

L'immagine e la scena. Bozzetti e figurini dall'archivio del Teatro La Fenice, 1938-1992, ed. by Maria Ida Biggi, Venezia, Marsilio, 1992;

Giuseppe Borsato scenografo alla Fenice, 1809-1823, ed. by Maria Ida Biggi, Venezia, Marsilio, 1995;

Francesco Bagnara scenografo alla Fenice, 1820-1839, ed. by Maria Ida Biggi, Venezia, Marsilio, 1996;

Giuseppe e Pietro Bertoja scenografi alla Fenice, 1840-1902, ed. by Maria Ida Biggi and Maria Teresa Muraro, Venezia, Marsilio, 1998;

Il concorso per la Fenice 1789-1790, by Maria Ida Biggi, Venezia, Marsilio, 1997;

I progetti per la ricostruzione del Teatro La Fenice, 1997, Venezia, Marsilio, 2000;

Teatro Malibran, ed. by Maria Ida Biggi and Giorgio Mangini, with essays of Giovanni Morelli and Cesare De Michelis, Venezia, Marsilio, 2001;

La Fenice 1792-1996. Il teatro, la musica, il pubblico, l'impresa, by Anna Laura Bellina and Michele Girardi, Venezia, Marsilio, 2003;

Il mito della fenice in Oriente e in Occidente, ed. by Francesco Zambon and Alessandro Grossato, Venezia, Marsilio, 2004;

Pier Luigi Pizzi alla Fenice, edited by Maria Ida Biggi, Venezia, Marsilio, 2005;

A Pier Luigi Pizzi. 80, edited by Maria Ida Biggi, Venezia, Amici della Fenice, 2010. 


\section{Fondazione Teatro La Fenice di Venezia}

"La Fenice prima dell'Opera», 2012

a cura di Michele Girardi - ISSN 2280-8116

Wolfgang Amadeus Mozart, Cosi fan tutte, 1, 176 pp. ess. mus.: saggi di Luca Fontana, Emanuele d'Angelo, Emanuele Bonomi

VINCENZO BelLINI, La sonnambula, 2, 150 pp. ess. mus.: saggi di Federico Fornoni, Michele Girardi, Emanuele Bonomi

Thomas AdĖs, Powde Her Face, 3, 150 pp. ess. mus.: saggi di Daniela Tortora, Philip Hensher, Valentina Brunetti, Emanuele Bonomi

Georges Bizet, Carmen, 4, 208 pp. ess. mus.: saggi di Michele Girardi, Tommaso Sabbatini, Emanuele Bonomi
"La Fenice prima dell'Opera», 2012-2013

a cura di Michele Girardi - ISSN 2280-8116

Giuseppe Verdi, Otello, 1, 174 pp. ess. mus.: saggi di Guido Paduano, Anselm Gerhard, Marco Marica, Francesco Micheli, Emanuele Bonomi

Richard Wagner, Tristan und Isolde, 2, 204 pp. ess. mus.: saggi di Virgilio Bernardoni, Guido Paduano, Riccardo Pecci 
Responsabile musicologico

Michele Girardi

Redazione

Michele Girardi, Elena Tonolo

con la collaborazione di

Pierangelo Conte

Progetto e realizzazione grafica

Marco Riccucci

Edizioni del Teatro La Fenice di Venezia

a cura dell'Ufficio stampa

\section{Supplemento a}

\section{La Fenice}

Notiziario di informazione musicale culturale e avvenimenti culturali della Fondazione Teatro La Fenice di Venezia

dir. resp. Cristiano Chiarot aut. trib. di Ve 10.4.1997

iscr. n. 1257, R.G. stampa

concessionarie per la pubblicità

A.P. Comunicazione

VeNet comunicazioni

finito di stampare

nel mese di novembre 2012

da L'Artegrafica S.n.c. - Casale sul Sile (TV) 


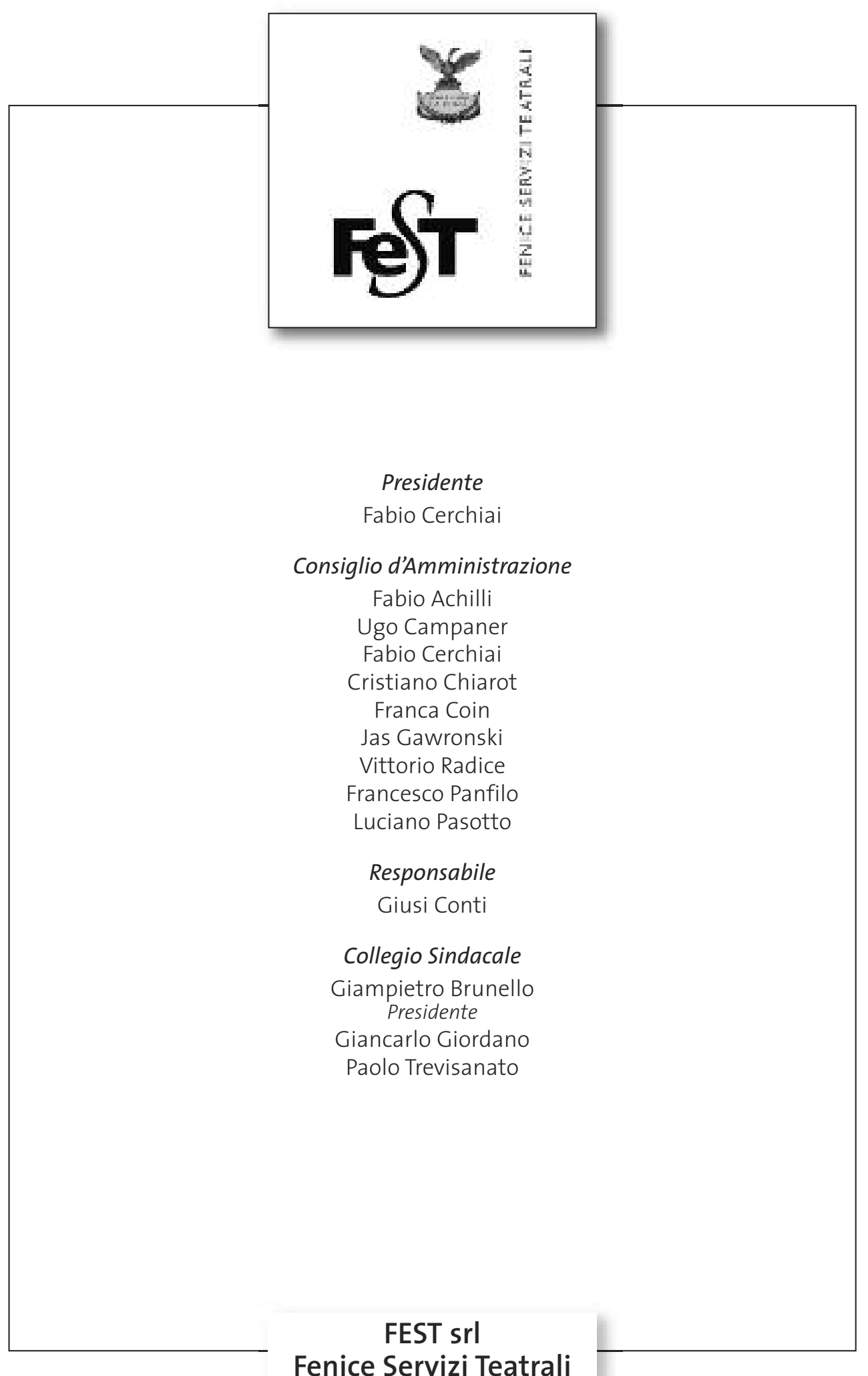



